\title{
THE CREATION, ANALYSIS, AND VERIFICATION OF A COMPREHENSIVE MODEL OF A MICRO ION THRUSTER
}

\author{
A Thesis \\ presented to \\ the Faculty of California Polytechnic State University, \\ San Luis Obispo
}

\author{
In Partial Fulfillment \\ of the Requirements for the Degree \\ Master of Science in Aerospace Engineering
}

by

Maxwell Bodnar

March 2016 
(C) 2016

Maxwell Bodnar

ALL RIGHTS RESERVED 


\section{COMMITTEE MEMBERSHIP}

TITLE:

The Creation, Analysis, and Verification of

a Comprehensive Model of a Micro Ion Thruster

AUTHOR:

Maxwell Bodnar

DATE SUBMITTED: $\quad$ March 2016

COMMITTEE CHAIR: Kira Abercromby, Ph.D.

Associate Professor of Aerospace Engineering

COMMITTEE MEMBER: Jordi Puig-Suari, Ph.D.

Professor of Aerospace Engineering

COMMITTEE MEMBER: David Marshall, Ph.D.

Professor of Aerospace Engineering

COMMITTEE MEMBER: Dan Goebel, Ph.D.

Senior Research Scientist, Jet Propulsion Laboratory 


\begin{abstract}
The Creation, Analysis, and Verification of a Comprehensive Model of a Micro Ion Thruster
\end{abstract}

\title{
Maxwell Bodnar
}

A computational model of the micro-ion thruster MiXI has been developed, analyzed, and partially verified. This model includes submodels that govern the physical, magnetic, electrostatic, plasma physics, and power deposition of the thruster. Over the past few years, theses have been conducted with the goal of running tests and analyzing the results; this model is used to understand how the thruster components interact so as to make predictions about, and allow for optimization of, the thruster operation. Testing is then performed on the thruster and the results are compared to the output of the code. The magnetic structure of the thruster was analyzed and numerous different configurations generated which were also evaluated by the optimizer and tested. Using the different configurations, models, and optimization tools, the total efficiency of the thruster is theoretically able to reach 69.4\%. Operational testing of the thruster at many different throttle settings demonstrated a maximum total efficiency of $45.9 \pm 24.6 \%$, discharge loss values as low as $109 \pm 25$ $\mathrm{eV} /$ ion, and total power required as low as $50.5 \pm 0.1 \mathrm{~W}$ to maintain thruster operation with beam extraction. Measurements of the plasma were taken using a Langmuir probe and the interpretation of the tests are used to verify the plasma physics submodel. Power draw measurements and analysis of the throttle inputs during testing are compared to the performance model outputs but were not accurate or consistent enough to fully verify the power deposition and plasma physics models. Analysis of the models and operational testing in this study have led to an increased understanding of the performance and operation of the MiXI-CP-V3 thruster, furthering the effort to create an efficient, flight capable micro-ion thruster. 


\section{ACKNOWLEDGMENTS}

I am very grateful for the help and support provided by my thesis advisor, Dr. Abercromby. Without her time, help, and expertise working in the lab, I would never have been able to finish this project. I am very glad for her assistance, teaching, and patience throughout

all my time at Cal Poly. I would also like to thank Dr. Goebel of JPL for all of his help throughout this thesis. His support and generosity in my times of need were invaluable. I also want to thank the space environment's lab technician Cody Thompson for his time and support during my work. Additionally, I want to thank all of the other teachers and faculty members at Cal Poly for their teachings through the years and for the education they have provided. Finally, I especially want to thank my parents, Mike and Marcia, for always being patient and supportive of my work and me; I am very thankful to have their love and support. 


\section{TABLE OF CONTENTS}

Page

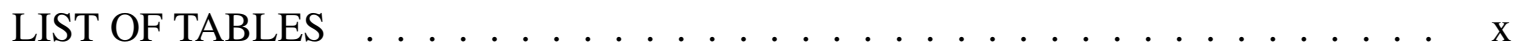

LIST OF FIGURES . . . . . . . . . . . . . . . . . . . xii

\section{CHAPTER}

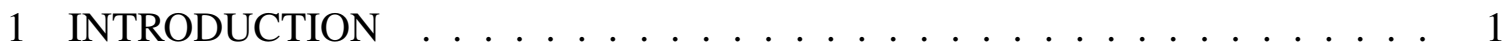

1.1 Ion Propulsion Overview . . . . . . . . . . . . . . . . 1

1.2 Miniature Xenon Ion Thruster $(\mathrm{MiXI}) \ldots \ldots \ldots$

1.2.1 MiXI Proposed Missions . . . . . . . . . . . . . . 4

1.3 Previous Work . . . . . . . . . . . . . . . 5

1.4 Motivation . . . . . . . . . . . . . . . . 10

1.5 Report Outline . . . . . . . . . . . . . . . . . 11

2 SYSTEM CONFIGURATION . . . . . . . . . . . . . . 13

2.1 Hollow Cathode . . . . . . . . . . . . . . . 13

2.1.1 Cathode Base: Propellant Attachment and Electrical Feed-through . 16

2.1.2 Propellant Line, Insert, and Heater Coil . . . . . . . . . . . . . 17

2.1.3 Propellant Isolator . . . . . . . . . . . . . . . 18

2.1 .4 Cathode Keeper . . . . . . . . . . . . . . . . . . 19

2.1 .5 Keeper Isolator . . . . . . . . . . . . . . . . 20

2.1.6 Cathode Chassis .................. 21

2.1 .7 Keeper Isolation Sheath . . . . . . . . . . . . . . . . 22

2.1.8 Hollow Cathode Assembly . . . . . . . . . . . . . . . 23

2.2 MiXI-CP-V3 Design . . . . . . . . . . . . . . . . . 24

2.2.1 Anode/Discharge Chamber . . . . . . . . . . . 25

2.2.2 Anode Magnet Mount . . . . . . . . . . . . . 26

2.2.3 Anode Cap Piece . . . . . . . . . . . . . . . . 27

2.2.4 Base Magnet Mount . . . . . . . . . . . . . . . . 28

2.2.5 Base Magnet Shield . . . . . . . . . . . . . . . . . 29

2.2 .6 Grid Isolation Mount . . . . . . . . . . . . . . . . . . . 30

2.2.7 Screen and Acceleration Grids . . . . . . . . . . . . . . . . 31 
2.2.8 MiXI-CP-V3 Assembly . . . . . . . . . . . . . . . . . 33

2.3 Final Assembly . . . . . . . . . . . . . . . . . . . . . . . 37

3 MAGNETIC MODEL CREATION AND ANALYSIS . . . . . . . . . . . . . . . 39

3.1 Magnet Selection . . . . . . . . . . . . . . . . . . 41

3.2 Magnetic Model Creation . . . . . . . . . . . . . . . . . . . . 42

3.2 .1 FEMM Overview . . . . . . . . . . . . . . . . . . . 43

3.2.2 FEMM Magnetics Model . . . . . . . . . . . . . . . . . 46

3.2.3 FEMM Electrostatic Model _ . . . . . . . . . . . . . . . 50

3.3 Magnetic Field Analysis _ . . . . . . . . . . . . . . . 52

3.3.1 Primary Electron Collision . . . . . . . . . . . . . . . 54

3.3.2 Plasma Potential . . . . . . . . . . . . . . . 56

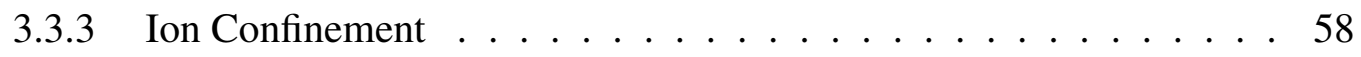

3.3.4 Other Considerations . . . . . . . . . . . . . . . . . . . 60

3.4 Magnetic Model Variations . . . . . . . . . . . . . . . . . . . . 62

3.4.1 Anode Cusp Spacer Ring . . . . . . . . . . . . . . . . . . 66

3.4.2 Base Magnet Spacer Ring _ . . . . . . . . . . . . . . 67

3.4.3 Base Magnet Offset Ring . . . . . . . . . . . . . . . . . 68

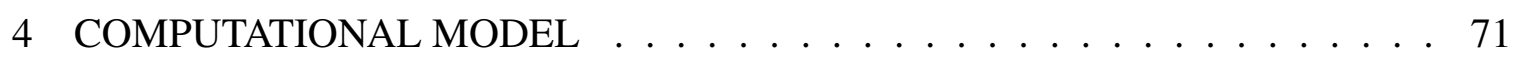

4.1 Optimization Code Overview . . . . . . . . . . . . . . . . . 72

4.1 .1 Constrained Inputs . . . . . . . . . . . . . . . . . 73

4.2 Performance Model . . . . . . . . . . . . . . . . . . . . . . . 75

4.2.1 Electron Temperature Sub-Model … . . . . . . . . . . 75

4.2.2 Plasma Discharge Sub-Model . . . . . . . . . . . . . . . . 84

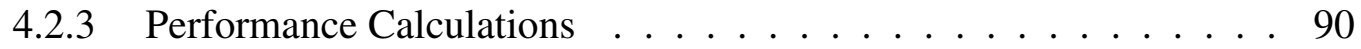

4.2.4 Power Deposition Sub-Model ．. . . . . . . . . . . . . . . 93

5 MODEL SOLUTIONS . . . . . . . . . . . . . . . . . . . 102

5.1 Performance Model Output . . . . . . . . . . . . . . . . . 102

5.2 Performance Curves . . . . . . . . . . . . . . . . . . . . . . . 106

5.3 Thruster Optimization . . . . . . . . . . . . . . . . . . . . . 114

5.3.1 Magnetic Field Optimization . . . . . . . . . . . . . . . 121

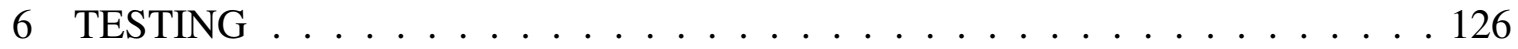

6.1 Experimental Apparatus $\ldots \ldots \ldots \ldots \ldots$ 
6.1 .1 Vacuum Chamber . . . . . . . . . . . . . . . . . 126

6.1.2 Electrical Configuration . . . . . . . . . . . . . . . . . . . 129

6.1 .3 Propellant System Configuration . . . . . . . . . . . . 133

6.2 Testing Procedure . . . . . . . . . . . . . . . . . . . . 134

6.2 .1 Data Acquisition . . . . . . . . . . . . . . . 135

6.2.2 Hollow Cathode Stand-Alone Test . . . . . . . . . . . . . . . . 137

6.2 .3 MiXI Testing . . . . . . . . . . . . . . . . . 138

7 RESULTS . . . . . . . . . . . . . . . . . . . . . . . . 142

7.1 Data Analysis . . . . . . . . . . . . . . . . . . . . . . 142

7.1.1 Results: Configuration_4o33s . . . . . . . . . . . . . 144

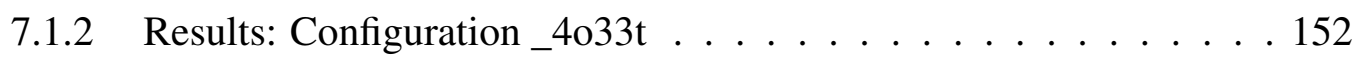

7.1.3 Results: Configuration_4o31s . . . . . . . . . . . 157

7.1.4 Results: Configuration_2o31s . . . . . . . . . . . . . 160

7.1.5 Numerical Error Analysis . . . . . . . . . . . . . . . . 162

7.2 Configuration and Performance Comparison . . . . . . . . . . . . 164

7.2.1 Performance Model Comparison . . . . . . . . . . . . . . . 168

7.3 MiXI Version Comparison . . . . . . . . . . . . . . . . . . . . 169

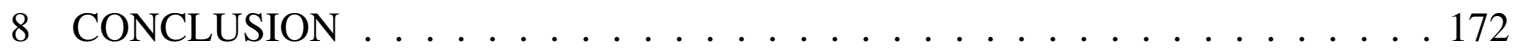

8.1 Future Work . . . . . . . . . . . . . . . . . . . . . 174

8.1 .1 Model Verification . . . . . . . . . . . . . . . . . . 176

8.1.2 Model Refinements . . . . . . . . . . . . . . . . . . . . . 176

BIBLIOGRAPHY . . . . . . . . . . . . . . . . . . . . . . . . . . . . 179 APPENDICES

A Lessons Learned . . . . . . . . . . . . . . . . . . . . 183

A.1 Hollow Cathode Assembly . . . . . . . . . . . . . . . . 183

A.2 Hollow Cathode Poisoning . . . . . . . . . . . . . 183

A.3 Electrical System . . . . . . . . . . . . . . . . . 185

A.4 Metal Deposition . . . . . . . . . . . . . . . . 185

B Vacuum Chamber Operating Procedures . . . . . . . . . . . 187

B.1 MAX Vacuum System Initial Setup and Safety Checks . . . . . 187

B.2 Cryogenic Vacuum System Setup . . . . . . . . . . 188

B.3 Experimental Loading Procedure $\ldots \ldots \ldots \ldots$ 
B.4 Chamber Roughing Procedure . . . . . . . . . . . . . . . . . 189

B.5 Cryogenic System Procedure . . . . . . . . . . . . . . . . . 190

B.6 Experimental Chamber Vacuum Operating Procedures . . . . . . 191

B.7 Experimental Unloading Procedure . . . . . . . . . . . . 192

B.8 Tips and Tricks . . . . . . . . . . . . . . . . . . . . . 193

B.8.1 Ionization Gauge DGAS . . . . . . . . . . . . . 195

B.8.2 Cryogenic Pump Regeneration Procedure . . . . . . . . 195

C Hollow Cathode Stand Alone Testing Procedures . . . . . . . . . . . . 197

C.1 Pre-Setup Procedures . . . . . . . . . . . . . . . . . . . . . . . . . 199

C.2 Hollow Cathode Assembly and Loading . . . . . . . . . . . . . 199

C.3 Propellant System Assembly . . . . . . . . . . . . . . . . . . 200

C.4 Electrical System Assembly _. . . . . . . . . . . . . . . . . 202

C.5 Hollow Cathode Operating Procedure . . . . . . . . . . . . 203

C.6 Troubleshooting Tips and Tricks . . . . . . . . . . . . . . 205

D MiXI Full Testing Procedures _ . . . . . . . . . . . . . . 207

D.1 MiXI Loading Procedure . . . . . . . . . . . . . . . 208

D.2 Propellant System Assembly . . . . . . . . . . . . . . . . . . 209

D.3 Electrical System Assembly . . . . . . . . . . . . . . . . . . . 209

D.4 MiXI Operational Procedure . . . . . . . . . . . . . . . 211

D.5 Troubleshooting Tips and Tricks . . . . . . . . . . . . . . 212

E Grid Convergence Study and Error Analysis . . . . . . . . . . . 214

E.1 Convergence Study Setup . . . . . . . . . . . . . . . . . 214

E.2 Convergence Analysis . . . . . . . . . . . . . . . . 217

E.3 Convergence Analysis Results _ . . . . . . . . . . . . . . 218

F Langmuir Probe Testing and Analysis . . . . . . . . . . . 220

F.1 Langmuir Probe Results . . . . . . . . . . . . . . . . 221 


\section{LIST OF TABLES}

Table

4.1 Power Deposition Model Sample Throttle Point and Required Outputs . . 99

4.2 Power Deposition Model Output Comparison . . . . . . . . . . . 100

5.1 Computational Model Sample Throttle Point _ . . . . . . . . . . 103

5.2 Computational Model Sample Throttle Point . . . . . . . . . . . . . . 104

5.3 Computational Model Throttle Point Bounds for Optimization . . . . . . 115

5.4 Throttle Level Result of Optimization on the Baseline Configuration, Maximizing Total Efficiency . . . . . . . . . . . . . . 116

5.5 Performance Model Output of Baseline Optimization, Maximizing Total Efficiency . . . . . . . . . . . . . . . . 117

5.6 Throttle Level Result of Optimization on the Baseline Configuration, Minimizing Dissipated Power . . . . . . . . . . . . . . . . . . 119

5.7 Performance Model Output of Baseline Optimization, Minimizing Dissipated Power . . . . . . . . . . . . . . . . 120

5.8 Throttle Level Result of Optimization on the 4i13s Configuration, Maximizing Total Efficiency . . . . . . . . . . . . . . . . 122

5.9 Performance Model Output of $4 i 13 s$ Optimization, Maximizing Total Ef-

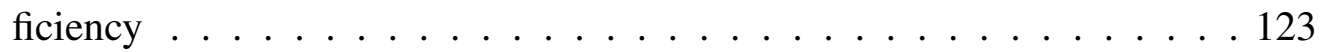

$6.1 \quad$ Power Supplies used in Operational Testing of MiXI . . . . . . . . . 133

6.2 Sources of Data Recorded from Operational Testing . . . . . . . . 136

6.3 Thruster Testing Throttle Levels . . . . . . . . . . . . . . . . . . . 139

7.1 Testing Results, Subset 1 from Configuration_4o33s . . . . . . . . 145

7.2 Testing Results, Subset 2 from Configuration_4o33s . . . . . . . . . 148

7.3 Testing Results, Subset 1 from Configuration_4o33t . . . . . . . . . 152

7.4 Testing Results, Subset 2 from Configuration_4o33t . . . . . . . . . . 154

7.5 Testing Results from Configuration_4o31s . . . . . . . . . . 158

7.6 Testing Results from Configuration_2031s . . . . . . . . . 160

7.7 Numerical Error Analysis Setup Values . . . . . . . . . . . . . 162

$7.8 \quad$ Numerical Error Analysis Results, Assuming Perfect $T_{s} \ldots \ldots 3$ 
7.9 Numerical Error Analysis Results, Assuming Perfect $T_{s} \ldots \ldots 3$

7.10 Testing Results Comparison Between Configurations . . . . . . . . . . 165

7.11 MiXI-CP-V3 Performance Parameters Comparison . . . . . . . . . . . 170

C.1 Hollow Cathode System Bill of Materials . . . . . . . . . . . . . . . . 198

C.2 Hollow Cathode Electrical System Bill of Materials _ . . . . . . . . . . 199

D.1 MiXI Additional Setup Equipment _ . . . . . . . . . . . . 207

E.1 Grid Convergence Analysis Setup . . . . . . . . . . . . . . 216

E.2 Grid Convergence Analysis Results . . . . . . . . . . . . . . . . 219 


\section{LIST OF FIGURES}

Figure

1.1 Ion Thruster Section View displaying the main components and steps to the operation of an ion thruster [6] . . . . . . . . . . . 2

1.2 Lunar Cubesat Design using MiXI as the main propulsion system [8] . . 4

1.3 Terrestrial Planet Finder Interferometer depiction utilizing MiXI as supplementary propulsion $[20] \ldots \ldots \ldots 5$

1.4 MiXI-CP-V1 Fully Assembled [42] . . . . . . . . . . . . . . . . 6

1.5 MiXI-CP-V2 Fully Assembled [18] . . . . . . . . . . . . . 7

1.6 MiXI-CP-V3 Fully Assembled [19] . . . . . . . . . . . . . . 8

1.7 Miniature Discharge Device Testbed used by Mao [22] . . . . . . . . . . 9

2.1 Hollow Cathode Cutaway showing the cathode tube, gas feed, heating coil, electron emitting insert, and two distinct plasma regions, all enclosed within the keeper electrode [12] . . . . . . . . . . . . . . 14

2.2 Miniature Internal Conduction Hollow Cathode [42] . . . . . . . . . 15

2.3 MiXI-CP-V3 Hollow Cathode Assembly Solid Model . . . . . . . . . 16

2.4 MiXI-CP-V3 Cathode Base Solid Model . . . . . . . . . . . . . . 17

2.5 MiXI-CP-V3 Propellant Line, Insert, and Heater Coil Solid Model . . 18

2.6 MiXI-CP-V3 Propellant Isolator Solid Model . . . . . . . . . . . . . . . 19

2.7 MiXI-CP-V3 Cathode Keeper Solid Model . . . . . . . . . . . . . 20

2.8 MiXI-CP-V3 Keeper Isolator Solid Model _ . . . . . . . . . . . 21

2.9 MiXI-CP-V3 Cathode Chassis Solid Model . . . . . . . . . . . . . 22

2.10 MiXI-CP-V3 Keeper Isolation Sheath Solid Model . . . . . . . . . . . . 23

2.11 MiXI-CP-V3 Hollow Cathode Assembly, Exploded View showing all of the components of the cathode assembly and required attachment equip-

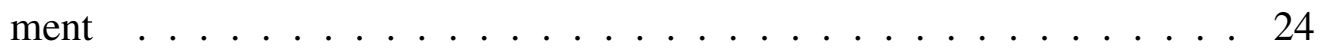

2.12 MiXI Discharge Chamber Fully Assembled Cutaway View . . . . . . 25

2.13 MiXI-CP-V3 Anode Solid Model . . . . . . . . . . . . . . . . 26

2.14 MiXI-CP-V3 Anode Magnet Mount Solid Model . . . . . . . . . . . . 27

2.15 MiXI-CP-V3 Anode Cap Piece Solid Model . . . . . . . . . . . . 28

2.16 MiXI-CP-V3 Base Magnet Mount Solid Model . . . . . . . . . . . . . . 29 
2.17 MiXI-CP-V3 Base Magnet Shield Solid Model . . . . . . . . . . . . 30

2.18 MiXI-CP-V3 Grid Isolation Mount Solid Model . . . . . . . . . . . . 31

2.19 MiXI Screen and Acceleration Grids _ . . . . . . . . . . . . . 32

2.20 Spacing of Screen and Acceleration Grids achieved via spacing washers . 33

2.21 MiXI Discharge Chamber Lower Section Exploded View _ . . . . . . 34

2.22 MiXI Discharge Chamber Upper Section Exploded View $\ldots \ldots$. . . 36

2.23 MiXI-CP-V3 Discharge Chamber Assembly . . . . . . . . . . . . 37

2.24 MiXI-CP-V3 Fully Assembled . . . . . . . . . . . . . . . . 38

3.1 MiXI-CP-V3 Magnetic Field Model from Knapp's Thesis [19] _. . . 40

3.2 MiXI solid model cutaway view showing the magnet location and orientation, baseline configuration . . . . . . . . . . . . 42

3.3 MiXI FEMM Model of the Thruster Structure Colorized and Annotated . 45

3.4 Baseline Configuration Mesh generated from FEMM . . . . . . . . 47

3.5 Magnetic Field Solution of the Baseline Configuration generated through

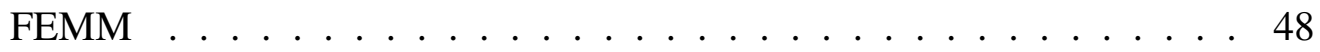

3.6 Magnetic Field Solution of Baseline Configuration displayed through

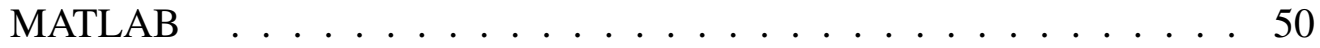

3.7 Electric Field of the Baseline Configuration Generated in FEMM _ . . 51

3.8 Electron Path in Plasma Discharge Chamber, NSTAR analog [38] . . . 53

3.9 Electron Path in Plasma Discharge Chamber, MiXI Thruster [38] . . . 54

3.10 Probability of Primary Electron Collision with Changing Magnetic Field

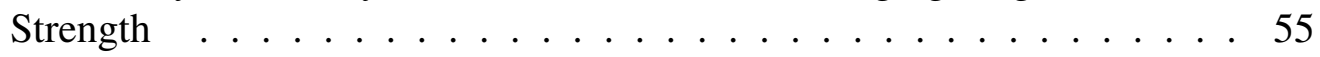

3.11 Plasma Potential Transition to a negative value with respect to the anode, resulting in lower primary electron energy $[12] \ldots \ldots$. . . . . 5 56

3.12 Plasma Potential with Changing Magnetic Field Strength _ . . . . . 57

3.13 Magnetic Field Strength within 6 Ring Cusp NSTAR Thruster highlighting the 60 Gauss closed contour $[15] \ldots \ldots$. . . . . . . . 58

3.14 Effect on Confinement Factor by changing Closed Loop Magnetic Field

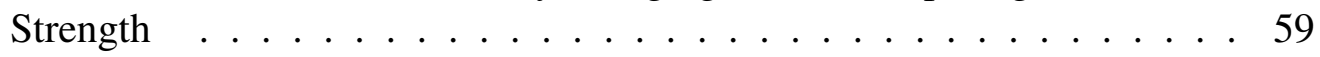

3.15 Magnetic Field Analysis of Configuration 4o33s, baseline . . . . . . 61

3.16 Magnetic Field Analysis of Configuration 2i31t . . . . . . . . . . . 62

3.17 Meshes of Two Sample Configurations _ . . . . . . . . . . . 65 
3.18 Anode Magnetic Ring Cusp Configuration Comparison between the baseline three magnet stack 3.18a and with the spacer installed 3.18b $\ldots 66$

3.19 Anode Cusp Spacer Ring with installed magnets . . . . . . . . . . 67

3.20 Base Magnetic Ring Configuration Comparison between the baseline four magnet stack $3.20 \mathrm{a}$ and with the spacer ring installed $3.20 \mathrm{~b} \ldots 68$

3.21 Base Magnet Offset Ring section view displaying the step cut, the actual design is a full ring . . . . . . . . . . . . . . . . . . . 69

3.22 Base Magnetic Ring Configuration Comparison between the baseline four magnet stack offset by the ring 3.22a and with the spacer ring also installed to only offset the remaining magnet pair $3.22 b \quad \ldots \ldots 69$

$4.1 \quad$ Functional flow chart of Optimization code $\ldots \ldots \ldots \ldots$

4.2 Ionization and Excitation Cross Sections for Xenon [12] . . . . . . 80

4.3 Current Distribution within the Discharge Chamber [15] . . . . . . . 85

4.4 Potential Distribution within the Discharge Chamber [15] . . . . . . 89

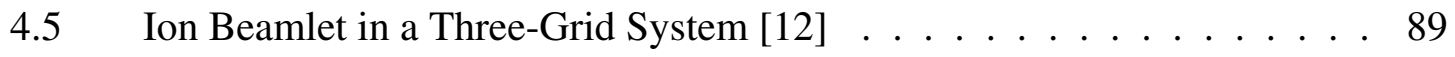

$5.1 \quad$ Performance Curve Example of an Ideal Thruster . . . . . . . . . 107

5.2 Performance Model Output: Discharge Loss vs. Mass Utilization Efficiency with Varying Beam Current; Discharge Voltage $=24 \mathrm{~V} \ldots \ldots 8$

5.3 Performance Model Output: Discharge Loss vs. Mass Utilization Efficiency with Varying Discharge Voltage; Beam Current $=0.2 \mathrm{~A} \ldots$. . . 109

5.4 Performance Model Output: Discharge Loss vs. Beam Current with Varying Mass Utilization Efficiency; Discharge Voltage $=24 \mathrm{~V} \ldots \ldots$

5.5 Performance Model Output: Discharge Loss vs. Beam Current with Varying Discharge Voltage; Mass Utilization $=0.85 \ldots \ldots \ldots$

5.6 Performance Model Output: Discharge Loss vs. Discharge Voltage with Varying Beam Current; Mass Utilization $=0.85 \ldots \ldots . \ldots 112$

5.7 Performance Model Output: Discharge Loss vs. Discharge Voltage with Varying Mass Utilization Efficiency; Beam Current $=0.2 \mathrm{~A} \ldots 113$

5.8 MATLAB Optimization Result of Baseline Configuration, Maximizing Total Efficiency . . . . . . . . . . . . . . . . 115

5.9 MATLAB Optimization Result of Baseline Configuration, Minimizing Power Dissipated . . . . . . . . . . . . . . . . . . . . . . . 119

5.10 Magnetic Field Analysis of Configuration 4i13s . . . . . . . . 124 
6.1 Minimum Atmospheric eXperimentation Chamber (MAX), Cal Poly Space Environments Laboratory . . . . . . . . . . . . . . . . . . 127

6.2 Schematic of MAX Vacuum Chamber and Pump Systems . . . . . . 128

6.3 Hollow Cathode Stand Alone Schematic and Electrical Diagram . . . . . 130

6.4 Electrical Schematic and Potential Change Diagram of a Three Grid Ion Thruster $[12] \ldots \ldots$. . . . . . . . . . . . . . . . . . . . . . . . . .

6.5 Electrical Schematic and Component Diagram of MiXI . . . . . . . . 132

6.6 Propellant System Setup Schematic ～. . . . . . . . . . . . . . . 134

6.7 Operation of the MiXI Cathode for the Hollow Cathode Stand-Alone

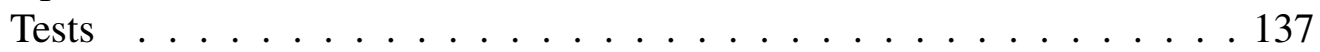

6.8 Operation of the MiXI thruster. Neutralizer CTFC disabled for picture purposes . . . . . . . . . . . . . . . . . 140

6.9 Langmuir Probe Sweep Sample, semi-log graph . . . . . . . . . . . 141

7.1 Data Analysis Performance Curve; Baseline Configuration, Discharge Voltage 25-30V . . . . . . . . . . . . . . . 150

7.2 Data Analysis Performance Curve; Baseline Configuration, Discharge

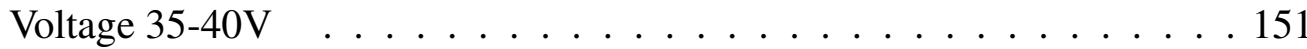

7.3 Data Analysis Performance Curve;_4o33t Configuration, Discharge Volt-

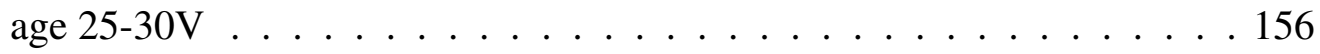

7.4 Data Analysis Performance Curve;_4o33t Configuration, Discharge Voltage $35-40 V$. . . . . . . . . . . . . . . . . . 157

7.5 Performance Model Comparison between Baseline and_4o33t Configurations. Beam Voltage 600V, Discharge Voltage Unconstrained . . . . . . 167

7.6 Testing Results and Performance Model Comparison; Baseline Configuration, Discharge Voltage 30-35V . . . . . . . . . . . . 168

C.1 Hollow Cathode Stand Alone Setup . . . . . . . . . . . . . . . 200

C.2 Propellant System Setup Schematic _ . . . . . . . . . . . . 201

C.3 Hollow Cathode Stand Alone Schematic and Electrical Diagram . . . . . 202

D.1 Electrical Schematic and Component Diagram of MiXI _ . . . . . 209

E.1 Grid Solution Space with Test Points _ . . . . . . . . . . 215

E.2 Grid Convergence Study results of two test points . . . . . . . . . 218

F.1 Langmuir Probe Sweep Raw Data . . . . . . . . . . . . . . . . 221 
F.2 Langmuir Probe Analysis of Ion Saturation Current _ . . . . . . . . 222

F.3 Langmuir Sweep of the Log of Current versus Voltage . . . . . . . . 223

F.4 Langmuir Probe Analysis of Electron Temperature and Electron Saturation Current . . . . . . . . . . . . . . . 223

F.5 Completed Langmuir Probe Analysis _ . . . . . . . . . . . . . . 224 
Chapter 1

INTRODUCTION

With the increasing complexity of space missions and the desire for sustainable human spaceflight, the development of high efficiency propulsion systems has been an important area of research. While Electric Propulsion (EP) systems provide less thrust than conventional chemical systems, they offer a significant increases in propulsive efficiency, thus reducing the propellant and propulsion system mass required and the total cost of a spacecraft. EP has been shown to be able to run reliably for years at a time [33] and complete missions that would have been impossible using a chemical system $[5,13]$.

Electric propulsion as a concept was first discussed in 1906 by Robert Goddard [11], and experimental ion thrusters were first launched in 1964 with SERT 1 [34]. It wasn't until 1971 when Russia launched the first spacecraft with an electric propulsion system to be used for flight purposes and not just as an experiment [3]. The first commercial use of electric propulsion in the United States was with a launch in 1997 with the Huges Xenon Ion Propulsion System (XIPS) [2]. Over the years, the US, Japan, the European Space Agency (ESA), and Russia have all launched new spacecraft with dozens of ion and hall thrusters.

\subsection{Ion Propulsion Overview}

As opposed to chemically oxidizing fuel to create energy and thrust, ion thrusters create thrust by accelerating ionized fuel particles using an electric potential field. There are three distinct types of electric propulsion systems: electrothermal (resistojet, arcjet), electrostatic (Hall effect, gridded ion), and electromagnetic (pulsed plasma, magnetohydrodynamic). There are three main stages to an ion thruster: electron production, propellant discharge and 
ionization, and ion acceleration. The way in which the electrons and plasma are produced and the ions are accelerated are specific to the type of thruster. The two most developed technologies for flight applications are gridded ion thrusters and Hall effect thrusters. The focus of this thesis will be on gridded ion thrusters, or just "ion thrusters".

Figure 1.1 is a cut-away of the NSTAR ring-cusp ion thruster, its components, and annotations of the steps involved in the operation an ion thruster.

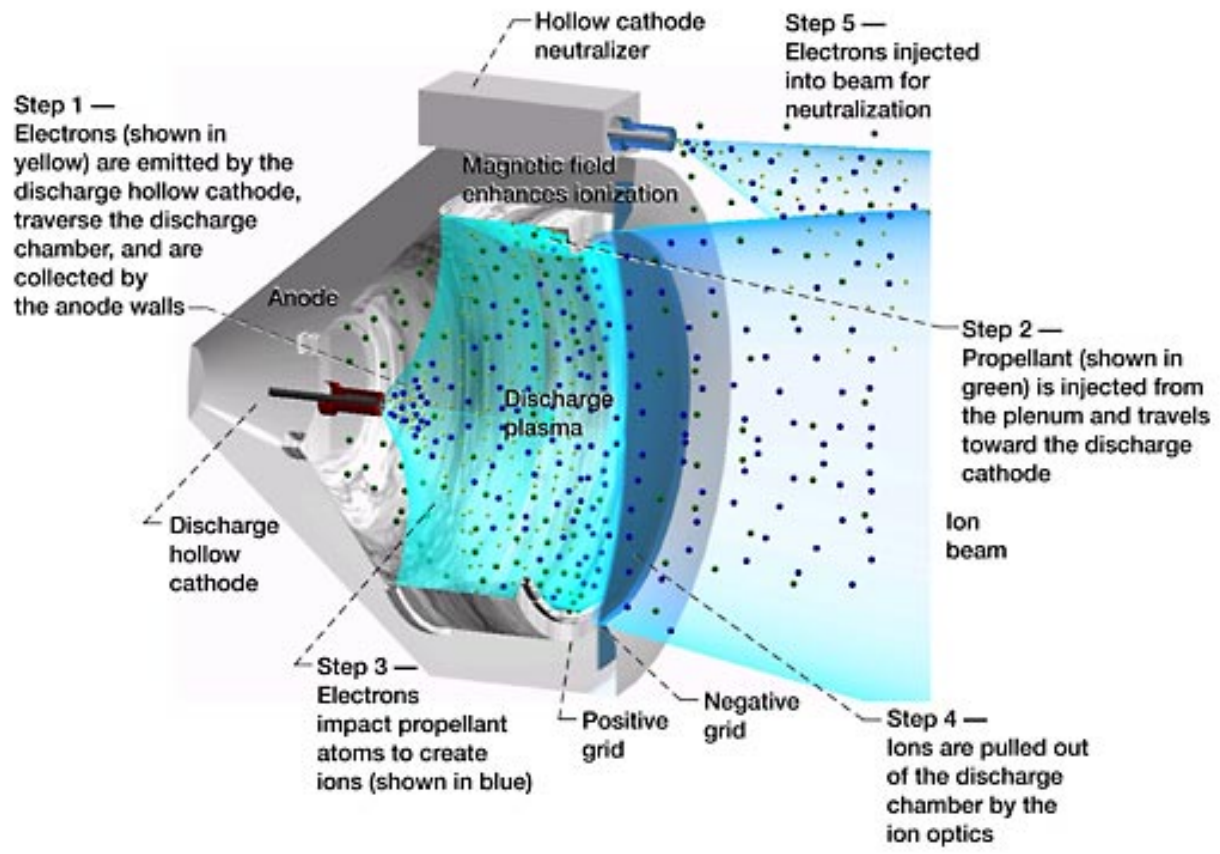

Figure 1.1: Ion Thruster Section View displaying the main components and steps to the operation of an ion thruster [6]

In this thruster, electrons are created through heating an insert within a hollow cathode. A neutral propellant, typically Xenon, is fed through the cathode and bombarded with electrons, at which point the ionization process begins. Within the discharge chamber, magnetic fields confine the electrons and allow for more ionization and plasma production. Gridded ion thrusters then accelerate the ions through a series of charged metal grids, creating an ion beam and providing thrust to the spacecraft. Gridded ion thrusters feature the 
highest efficiency (from $>60 \%$ to $>80 \%$ ) and a very high specific impulse (from 2000 to over 10,000 sec) compared to other ion thruster types [37].

EP systems have been used for orbit raising, station keeping, and high demand science missions that would have been otherwise not been possible with chemical systems [5, 31]. Successes with gridded ion and Hall effect thrusters have led to an increase in research pertaining to ion thrusters to fit specific needs. A number of small, low power, low thrust, and high efficiency propulsion systems have been designed and tested over the past few years, many of which have been proposed for use on missions requiring fine attitude adjustments or formation flying.

\subsection{Miniature Xenon Ion Thruster (MiXI)}

The Miniture Xenon Ion thruster (MiXI), first developed by Richard Wirz in 2005, is an ion thruster with a $3 \mathrm{~cm}$ diameter discharge chamber. It was capable of producing thrust on the order of $1 \mathrm{mN}$ with a specific impulse of over $3000 \mathrm{~s}$ [38]. Major characteristics of his design were that it was three centimeters in diameter with a diameter:length ratio of one, had a three ring-cusp magnetic field confinement design, and had a coiled tungsten filament cathode (CTFC) to initiate plasma generation. This design was adapted from larger ion thrusters such as XIPS and NSTAR in an attempt to utilize the same designs on a much smaller system. His design was able to achieve stable discharge and produce $1.429 \mathrm{mN}$ with $80 \%$ mass utilization efficiency while using less than $50 \mathrm{~W}$ of power.

With the success of the first micro ion thruster, several proposed satellite missions have considered implementing MiXI as a form of micro propulsion while in orbit. The small thrust and low power requirements make it an ideal candidate for missions requiring precise attitude adjustments or close proximity formation flight. 


\subsubsection{MiXI Proposed Missions}

MiXI has been considered as the primary propulsion system for CubeSat missions requiring high delta-V on large, multiple-cube buses using deployable arrays. The feasibility of a lunar CubeSat mission utilizing the MiXI thruster as the primary propulsion system was investigated by Conversano and Wirz [8]. Traditionally, CubeSats are a 10cm cube with a mass no more than $1 \mathrm{~kg}$ representing $1 \mathrm{U}$ (1 Unit). This mission would use a $3 \mathrm{U}$ frame with deployable solar panels and internal components similar to larger satellites that a $1 \mathrm{U}$ system normally wouldn't have. This $3 \mathrm{U}$ CubeSat would be capable of powering and utilizing a 40W micro ion thruster to achieve over $7000 \mathrm{~m} / \mathrm{s}$ of delta- $\mathrm{V}$ for this lunar mission.

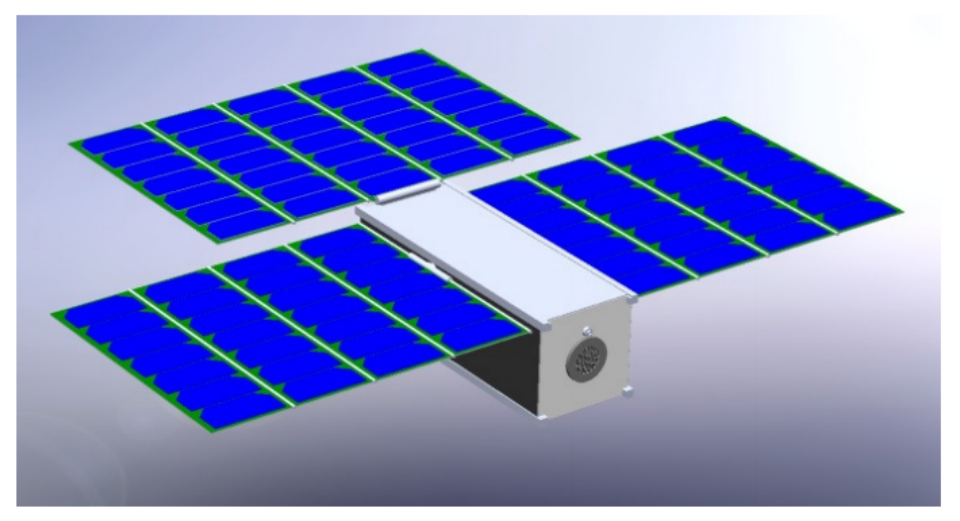

Figure 1.2: Lunar Cubesat Design using MiXI as the main propulsion system [8]

Another mission proposed to use MiXI thrusters on board multiple satellites is the Terrestrial Planet Finder (TPF) [20]. This mission utilizes a collector spacecraft with an optics package and a collector array of four other spacecraft. These five spacecraft will need to keep a very precise formation during flight to operate correctly and strict attitude control. The RCS system for these spacecraft was determined to need an ion thruster with an Isp of 2500-3500 s and be capable of more than $750 \mathrm{~m} / \mathrm{s}$ of delta-V. Further analysis lead to the proposition of using several ion thruster pods containing several MiXI thrusters to achieve the necessary attitude adjustment and formation flight. 


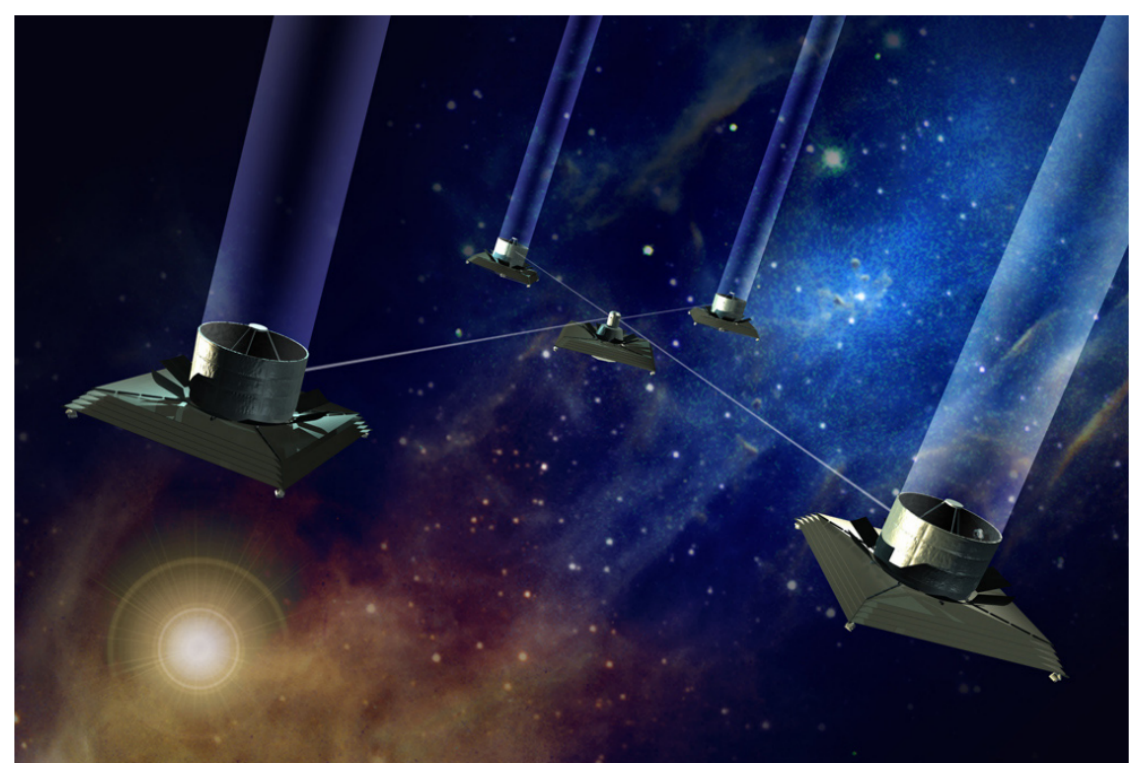

Figure 1.3: Terrestrial Planet Finder Interferometer depiction utilizing MiXI as supplementary propulsion [20]

\subsection{Previous Work}

Design on MiXI has continued and evolved over the years after having started in 2005 with Dr Wirz. Work with MiXI began at California Polytechnic University San Luis Obispo (Cal Poly) in 2010 with Younger's thesis designing and validating a thermal model for MiXI [42]. The thruster was redesigned and modeled using SolidWorks and analyzed using the SolidWorks thermal modeling software package. Younger then performed tests to validate the thermal model. The resulting iteration was called MiXI-CP-V1, shown in Figure 1.4. 


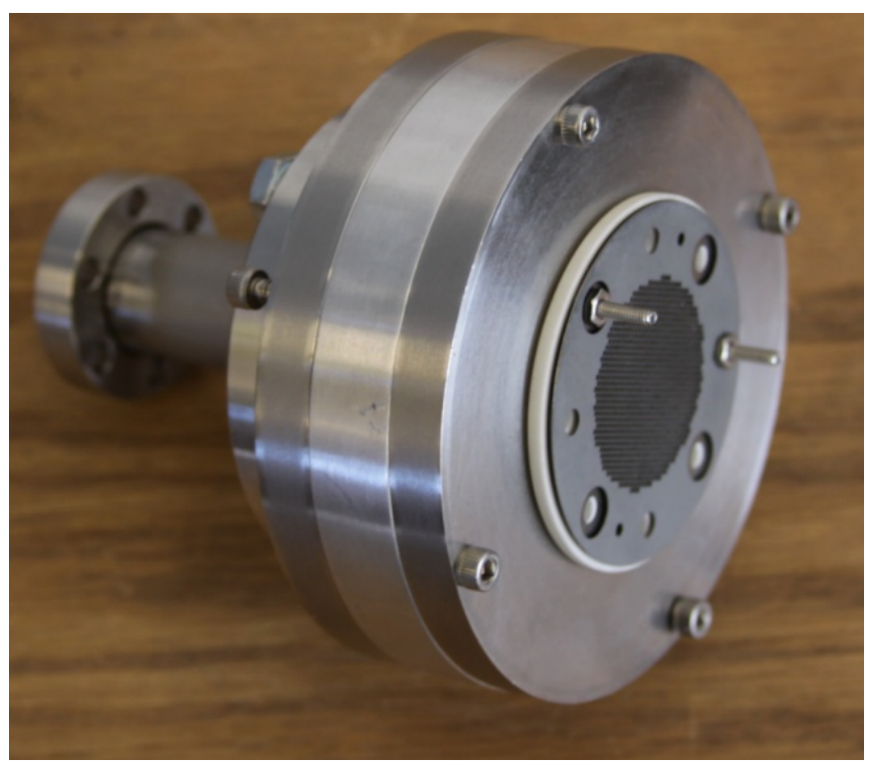

Figure 1.4: MiXI-CP-V1 Fully Assembled [42]

Cal Poly continued work on the thruster in 2011 when a student, David Knapp, replicated MiXI for use as an academic tool and testbed at Cal Poly. The MiXI-CP-V2 was very similar to the original design by Wirz in that it was still a three-ring-cusp design and utilized a CTFC for electron bombardment [18]. This version was successfully tested at Cal Poly's facilities and was able to achieve 0.3-0.7 mN of thrust, 1800-3500 s Isp, with about $15-40 \%$ mass utilization efficiency. Argon was used as a cheaper propellant and was presupposed as the leading cause for the lower thrust and efficiency seen from this thruster model. Additionally, Knapp specified that a more in depth analysis of the magnetic field design and electron confinement could have improved the overall performance. The thruster MiXI-CP-V2 is shown in Figure 1.5. 


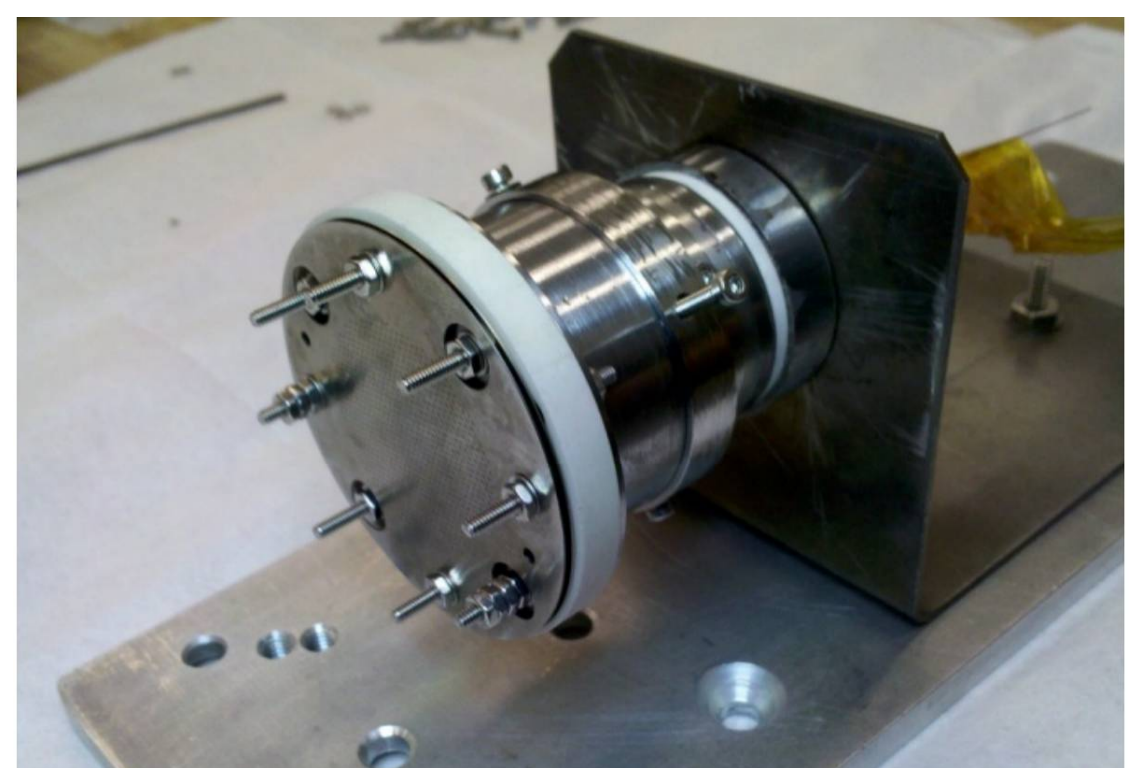

Figure 1.5: MiXI-CP-V2 Fully Assembled [18]

In 2012, Knapp continued the redesign of MiXI with the implementation of an orificed hollow cathode. All previous versions of the thruster were designed around CTFC's for electron bombardment. The goal was to replace the CTFC and redesign the thruster to improve the thrust and performance of the thruster. This design was called MiXI-CP-V3 and was able to produce 1.2-2.0 $\mathrm{mN}$ of thrust at an Isp of between 1500-3300 $\mathrm{s}$ and a mass utilization efficiency of 35-75\% [19]. Testing revealed that the thruster could be run with a hollow cathode and observations from the study have led to a greater understanding of the operation of the MiXI thruster. 


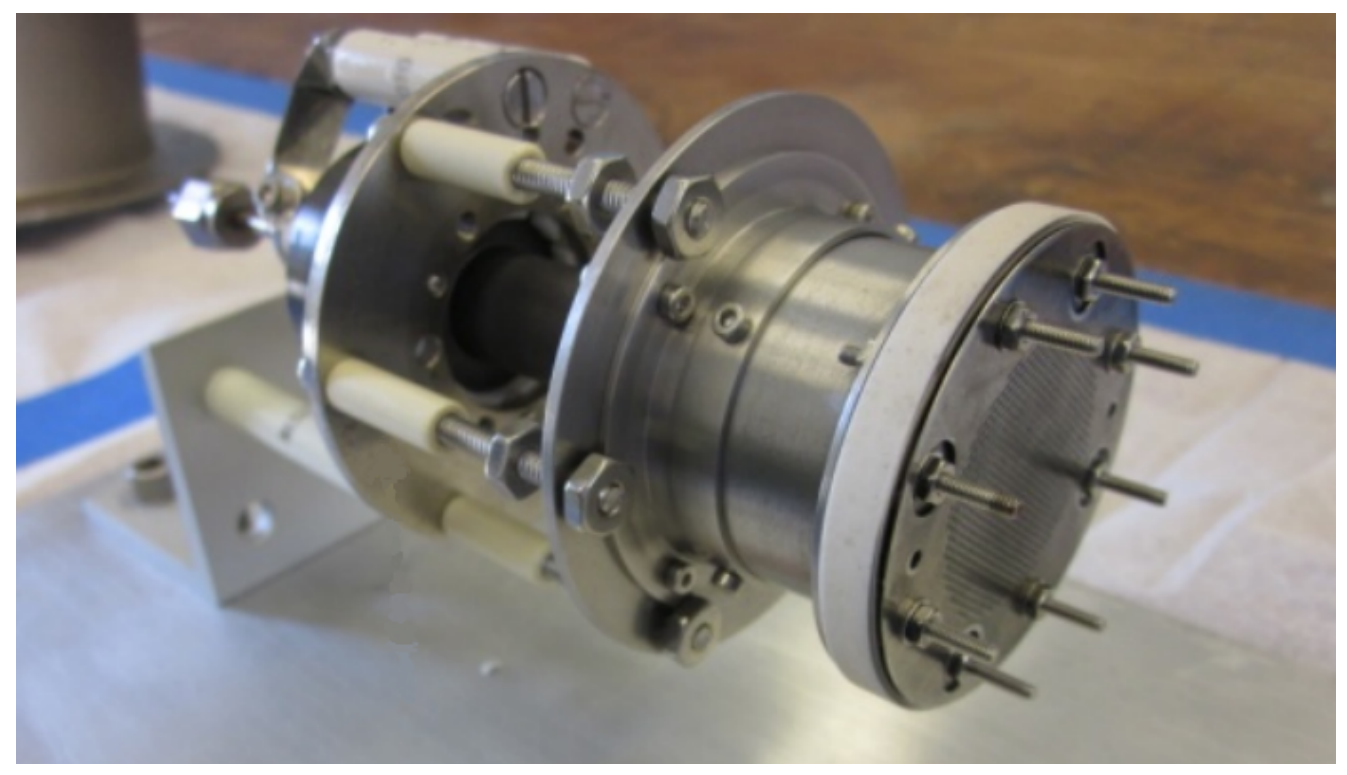

Figure 1.6: MiXI-CP-V3 Fully Assembled [19]

In 2013, UCLA's Hann Mao studied fundamental plasma discharge behavior within miniature ring-cusp ion thrusters and the effects of decreased volume on the plasma structure [22]. A research model was fabricated to represent the plasma generation chamber of a miniature ion thruster. The plasma density and spacial variations of the plasma parameters were measured using a Langmuir probe. The magnetic confinement strength was varied and its effects on the plasma parameters explored. The results of his study were applied to develop a design tool for miniature discharges and to redesign the experimental device to provide a $57 \%$ improvement in beam current over the original configuration. The miniature discharge device testbed used by Mao is shown in Figure 1.7. 


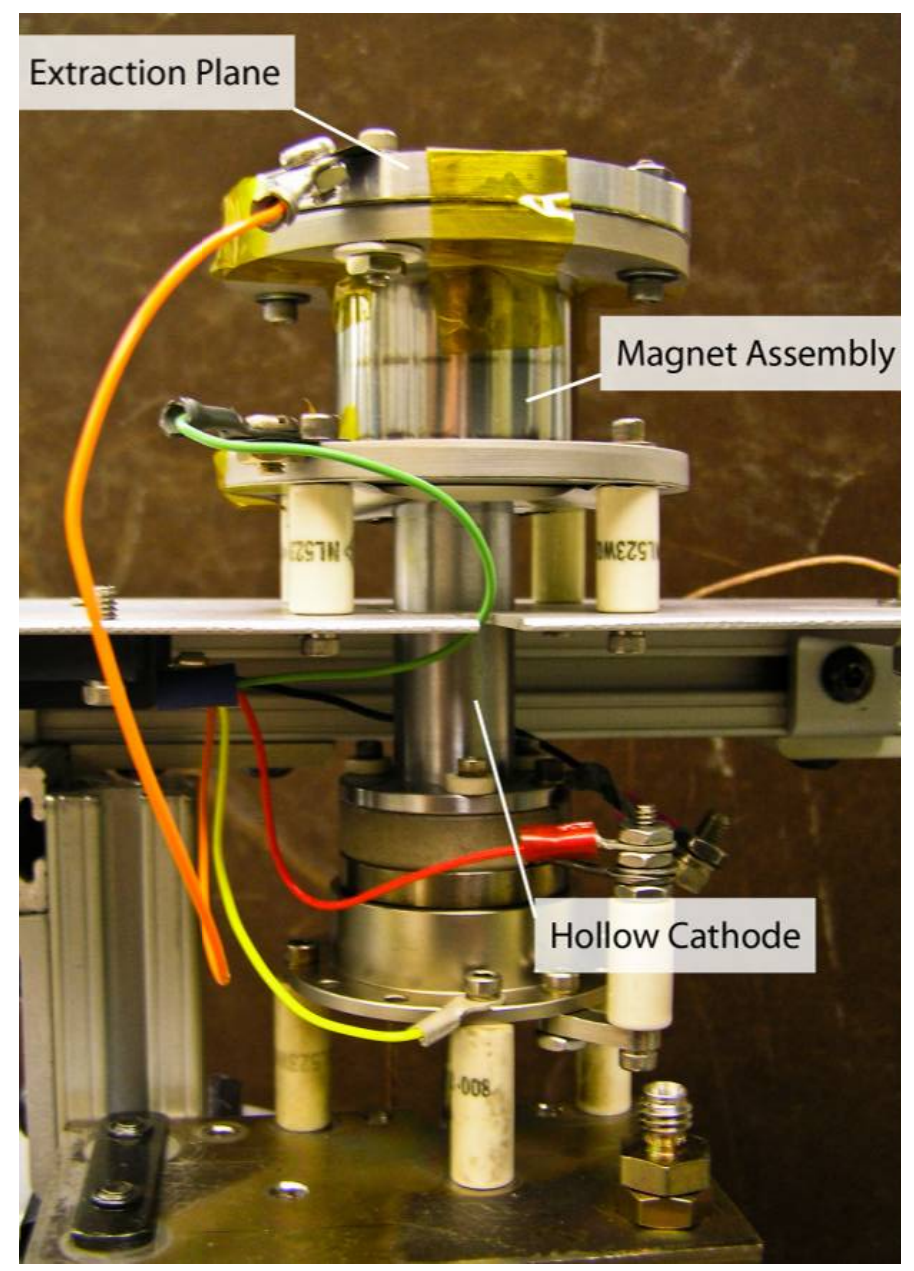

Figure 1.7: Miniature Discharge Device Testbed used by Mao [22]

In 2014, Cal Poly's Samuel Parker continued research on MiXI with the goal of better understanding the thermal aspect of the thruster. The operation of MiXI with a hollow cathode has proven troublesome in that the thruster will overheat rather easily $[19,22]$ and is not able to achieve steady state operation in its current form. Parker's goal was to develop a thermal model in Ansys FLUENT to analyze the amount of heating in the thruster and determine how to mitigate the thermal load on the magnets to postpone demagnetization. While a single solution to prevent demagnetization and significantly improve thruster runtime was not discovered, insight into the thermal environment of the MiXI thruster has proven helpful in the goal to create a flight capable miniature ion thruster. 


\subsection{Motivation}

The overarching goal of the previous work completed on the design of MiXI was to create an efficient and flight capable miniature ion thruster. There have been challenges in achieving a system that can run continuously utilizing the hollow cathode design. Sub-optimal magnet configuration, poor thermal dissipation, and an incomplete understanding of the plasma parameters within the thruster have led to inefficient designs in terms of power and mass utilization efficiency [19, 38, 22] and thermal deposition [30, 42].

The goal of this thesis was to take an analytical approach to the design of an ion thruster that would consider the hollow cathode effects, electromagnetics model, plasma discharge model, ion optics, thermal modeling, and power deposition models to find an optimized ion thruster design based upon constrained inputs and a given geometry. This approach is intended to be modular in that specific sections of the process can be refined and replaced with more advanced models within this framework. The goal with this comprehensive model is to gain insight to the specifics of the design and configuration so that advancements to performance can be achieved.

Specifically to the efforts at Cal Poly, the goal was to redesign the thruster and identify operational parameters to achieve stable and steady state operation of MiXI. In the previous state, the thruster was not able to run to steady state due to thermal problems; the thruster was being run with excess power and at inefficient settings. Using the processes developed in this thesis, the thruster was able to be reconfigured and steady state operation of the thruster was achieved at some throttle levels.

While the model and approach of this thesis were developed for MiXI, it is the hope that this approach can be used on all sizes and configurations of ion thrusters. With refinements to the sub-models, a greater understanding of all of the internal processes should be 
achievable and optimization based on spacecraft specifics and mission parameters can be performed on all types of thrusters.

\subsection{Report Outline}

A small scale Xenon ion thruster has already been demonstrated in the laboratory environment, but the plasma discharge processes and steady state thermal solution are not well known. Improvements to the confinement and discharge of the plasma would greatly improve the operation and application of the thruster. The current study approaches the design of an ion thruster from a purely analytical approach. This is done in order to obtain an optimized and converging solution of the inputs and structure of the thruster.

Chapter 2 presents the components of the MiXI thruster and discusses the assembly of the system. A complete solid model of the system was created and the parts of the hollow cathode and full thruster assembly are shown.

Chapter 3 discusses the magnetic field creation and analysis within the discharge chamber. Other reports have identified that the design of the magnetic field is a very critical aspect to the configuration of the thruster $[38,19,22]$ and all have suggested that further refinement to the magnetic field model may benefit thruster operation and performance. The processes of developing the magnetic field solution and the results are shown. The different physical configurations and the magnetic solution from those configurations are also discussed. The results in this section are used in the analytical equations and as a basis of discussion on improving the magnetic field design.

Chapter 4 discusses the computational model developed and used in this study, including all of the analytical equations and processes used to determine the operation of the thruster configuration. This section introduces the plasma processes present in the discharge chamber and highlights some of the difficulties encountered with the analysis. 
Chapter 5 presents the solutions to the plasma discharge model developed in Chapter 4. The method of optimization and convergence upon solutions of the model is discussed and analyzed. The model is also adapted to use the current MiXI physical designs to optimize operation and power consumption.

Chapter 6 discusses the testing procedure and experimental setup of the MiXI thruster. The cathode was run independently to determine minimum power and other requirements that were used as inputs in some parts of the computational model. Measurements were taken of the plasma discharge using a Langmuir probe and compared to theoretical values. Numerous tests were conducted that were compared with the analytical solutions developed in Chapter 5.

Chapter 7 presents the results from testing the MiXI truster. The test data is compared to the outputs from the computational model. The differences between the performance model theory and test results are discussed.

The final Chapter 8 compares the experimental and theoretical cases and identifies areas of concern. The major findings of the study are presented along with specific areas of improvement and suggestions of future work. 
Chapter 2

\section{SYSTEM CONFIGURATION}

The thruster used for testing and design verification in this thesis was the MiXI-CP-V3 thruster, briefly discussed in the introduction. This thruster was based on the design developed by Wirz and features a $3 \mathrm{~cm}$ discharge chamber and a length:diameter ratio of 1 specified by the MiXI design for performance [38]. MiXI-CP-V3 greatly deviates from the previous designs in that it uses a hollow cathode assembly to facilitate electron bombardment [19]. The following subsections will describe the physical system and highlight important aspects of the parts that make up MiXI-CP-V3.

\subsection{Hollow Cathode}

Previous MiXI designs have all used Coiled Tungsten Filament Cathodes (CTFC) as the primary form of electron bombardment. CTFC's are made from a coiled wire of tungsten and emit electrons as current is passed through and heats up the wire. Tungsten has a relatively high work function of $4.55 \mathrm{eV}$ and must be heated to over $2600 \operatorname{deg} C$ to emit electrons with enough energy to begin propellant ionization $[12,38,42]$. Due to the large amount of energy and the high temperatures required by the CTFC, they are less efficient and can lead to excessive heating of the discharge chamber and the electron confinement magnets.

These problems, among others, were alleviated by the development of hollow cathodes. A hollow cathode consists of a hollow tube with an embedded heater, an electron emitting insert, and an orifice plate on the downstream end. The cylindrical insert is the active electron emitter and can be made of several different materials that provide a low work

function surface in contact with the cathode plasma. The heater wraps around the insert to 
raise the temperature in order to begin electron emission. The electrons emitted from the insert ionize the propellant gas injected through the hollow cathode tube and form plasma. The electrons are then extracted through the orifice and into the thruster plasma where further ionization and plasma generation occurs.

A cutaway schematic of a hollow cathode is presented in Figure 2.1. From this image, it is easy to see the components of the hollow cathode and the regions of plasma within the assembly.

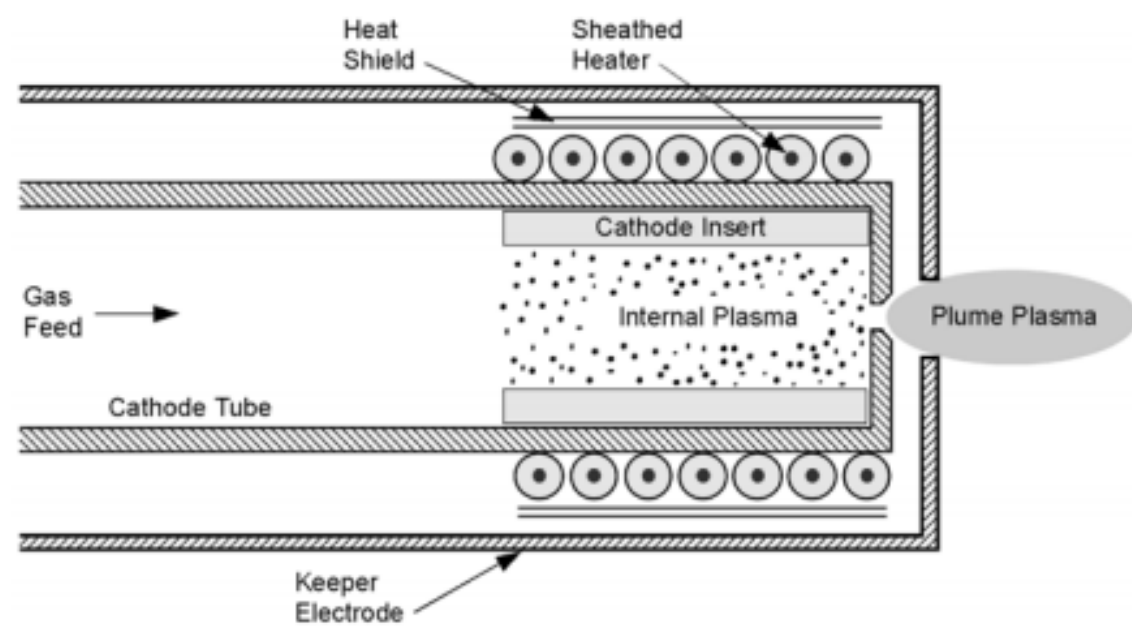

Figure 2.1: Hollow Cathode Cutaway showing the cathode tube, gas feed, heating coil, electron emitting insert, and two distinct plasma regions, all enclosed within the keeper electrode [12]

Development of miniature hollow cathodes allows for the creation of a small robust cathode designed for low propellant flow rates and low discharge current operation [40]. One such hollow cathode initially designed for used in small ion thrusters is an internal conduction cathode, shown in Figure 2.2. 


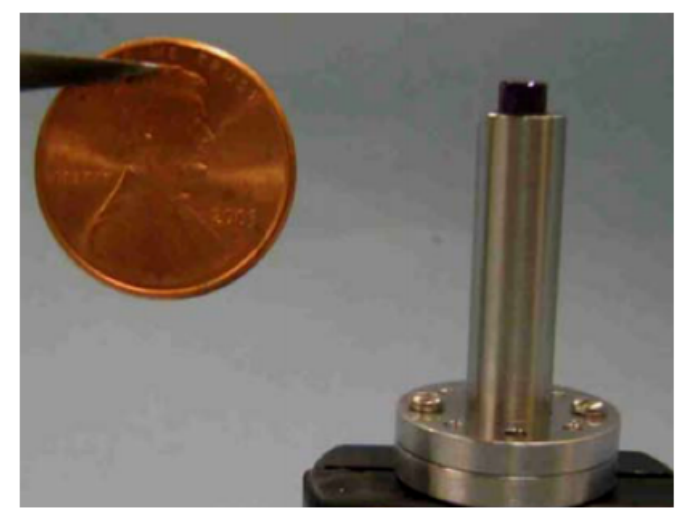

Figure 2.2: Miniature Internal Conduction Hollow Cathode [42]

The cathode installed in MiXI-CP-V3 is a version of a $6.25 \mathrm{~mm}$ diameter cathode similar in design to those used in 30cm NSTAR ion thrusters [19]. This cathode contains a tungsten impregnated barium oxide $(\mathrm{BaO}-W)$ insert. The barium oxide insert has a lower work function compared to pure tungsten or similar inserts like lanthanum hexaboride $\left(\mathrm{LaB}_{6}\right)$ [12]. The $\mathrm{BaO}-\mathrm{W}$ insert in this hollow cathode requires a temperature over $1100 \operatorname{deg} C$ to achieve electron emission compared to $L a B_{6}$ which requires between 1300 and $1500 \operatorname{deg} C$. While requiring lower temperature, the $B a O-W$ is more susceptible to poisoning from impurities in the propellant gas and has a higher evaporation rate during operation when compared to $\mathrm{LaB}_{6}$ cathodes [12]. It was therefore required to keep a contaminate-free operational environment and use Propulsion Grade propellants with a purity of $99.9995 \%$ to maintain stable cathode operation.

The initial testing and installation of the $6.25 \mathrm{~mm}$ hollow cathode was performed by Knapp [19]. In his thesis, the cathode was successfully installed and tested and determined to be able to run stably at flow rates less than 1 SCCM and with discharge currents less than 1 A. Further testing into the low power and operation of the thruster was performed in this thesis with the goal to reduce the total power needed while maintaining stable discharge to increase the total efficiency of the thruster. 
An assembly showing the major components of the cathode is shown in Figure 2.3. The major components will be discussed in detail in the following subsections.

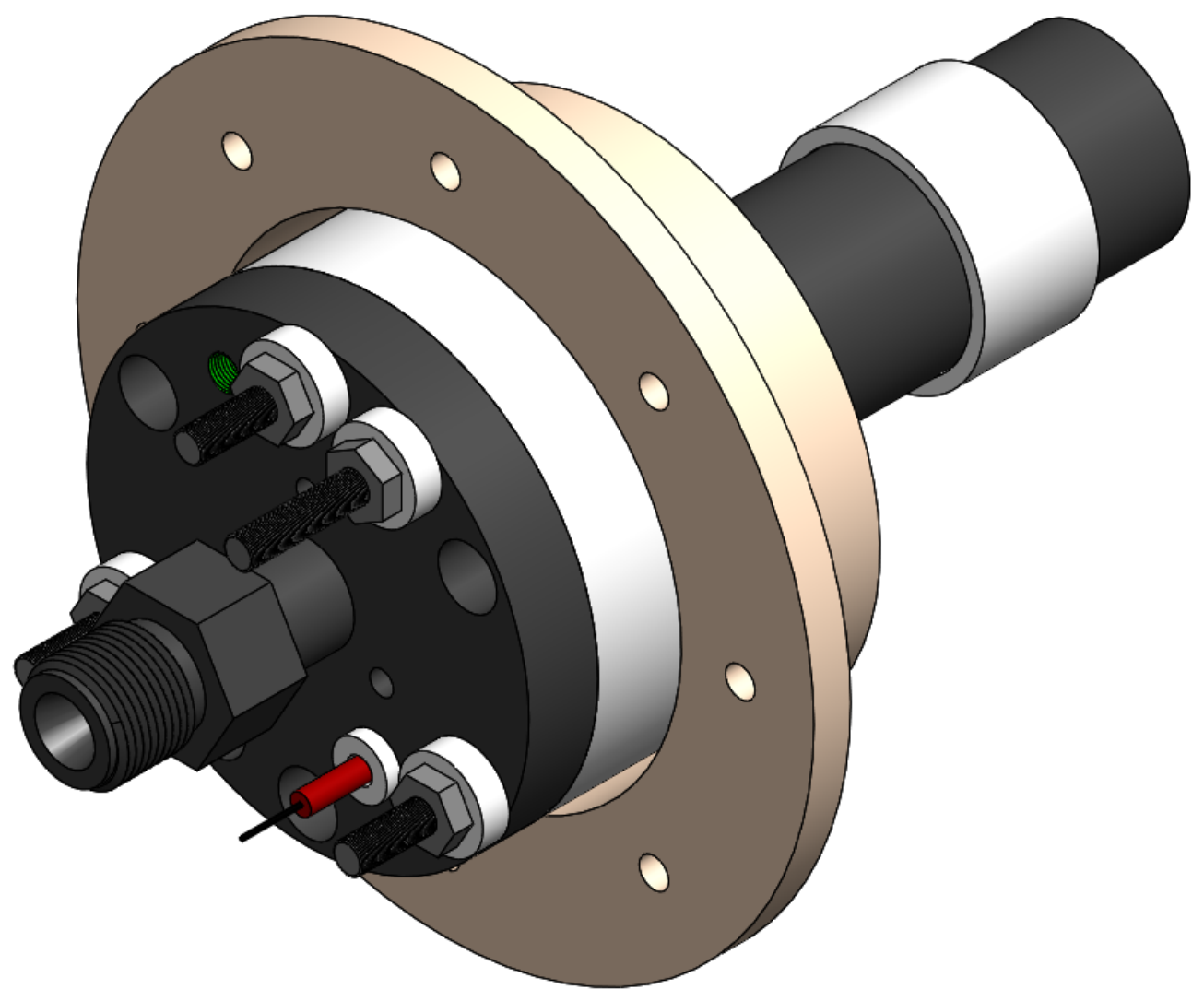

Figure 2.3: MiXI-CP-V3 Hollow Cathode Assembly Solid Model

\subsubsection{Cathode Base: Propellant Attachment and Electrical Feed-through}

The base of the cathode is made from an anodized stainless steel component that attaches to the fuel line and provides the attachment point for all the other components of the hollow cathode. The base is $38.1 \mathrm{~mm}$ in diameter and $6 \mathrm{~mm}$ thick with an extrude to a hex and a threaded Swagelok compatible section to attach the fuel inlet. There are two arrays of holes: a set of three $2 \mathrm{~mm}$ threaded holes in a $14.65 \mathrm{~mm}$ circle to attach the propellant tube, and a set of eight $5.1 \mathrm{~mm}$ diameter through-holes in a $31.75 \mathrm{~mm}$ circle to facilitate the attachment 
to other parts. There are two other holes that provide a threaded ground attachment point and a through hole for the heater. A solid model representation of the cathode base is shown in Figure 2.4.

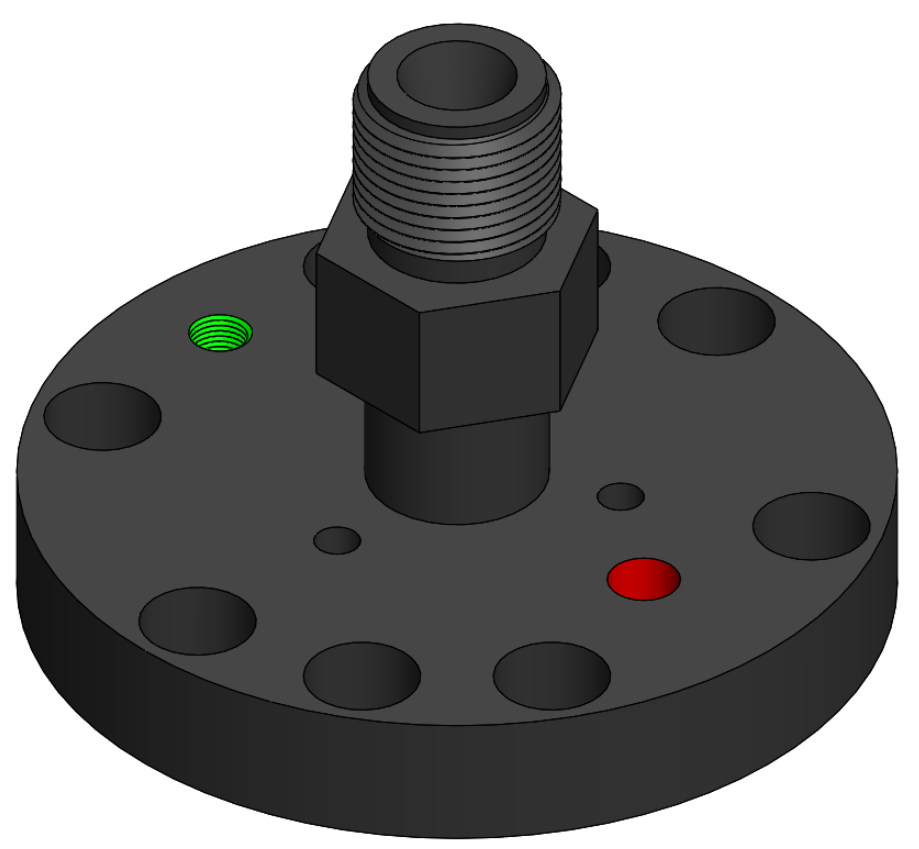

Figure 2.4: MiXI-CP-V3 Cathode Base Solid Model

\subsubsection{Propellant Line, Insert, and Heater Coil}

The propellant line houses the electron emitting insert and is enclosed on one end in the heater coil and heat shield. The propellant line is a hollow tube $55.4 \mathrm{mmm}$ long and $6.35 \mathrm{mmm}$ in diameter with an internal diameter of $5.08 \mathrm{~mm}$; the base of the propellant line has a flange that is $19.05 \mathrm{~mm}$ in diameter and $3.56 \mathrm{~mm}$ thick with an array of 3 throughholes to mate with the threaded holes in the cathode base. The downstream end of the tube has an orifice with a $1.25 \mathrm{~mm}$ opening.

Embedded in the propellant line is the $\mathrm{BaO}-W$ insert that is pressed against the orificed end. Surrounding the propellant line is a coiled ceramic insert heater which is welded to the outside of the propellant line. The heater is covered by a dimpled tantalum multi-layer 
heat shield. A solid model representation of the propellant line and associated components is shown in Figure 2.5.

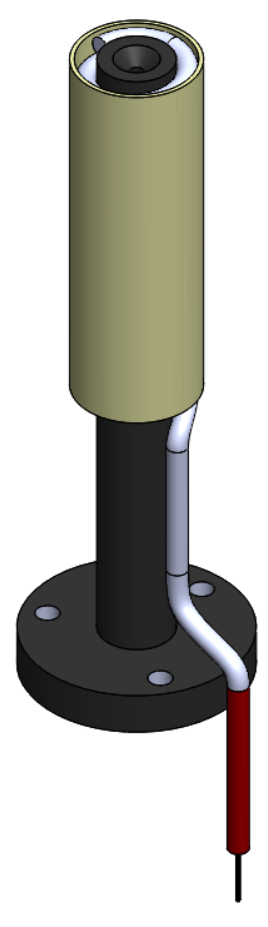

(a) MiXI-CP-V3 Propellant Line Solid Model

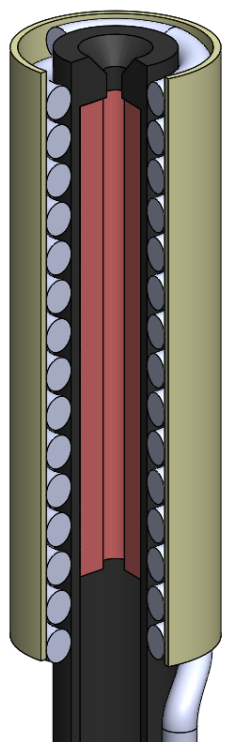

(b) Propellant Line Model Cutaway View

Figure 2.5: MiXI-CP-V3 Propellant Line, Insert, and Heater Coil Solid Model

Figure $2.5 \mathrm{~b}$ shows a cutaway view of the heater and insert within the propellant line.

\subsubsection{Propellant Isolator}

A thick cylindrical ceramic piece is used to isolate the propellant base from the keeper. The propellant isolator has a $38.1 \mathrm{~mm}$ outer diameter, a $25.4 \mathrm{~mm}$ inner diameter, and is $11.4 \mathrm{~mm}$ tall. There are also four $5.1 \mathrm{~mm}$ diameter through-holes centered on a $31.75 \mathrm{~mm}$ circle; these holes align with the through-holes in the cathode base. A solid model representation of the propellant isolator is shown in Figure 2.6. 


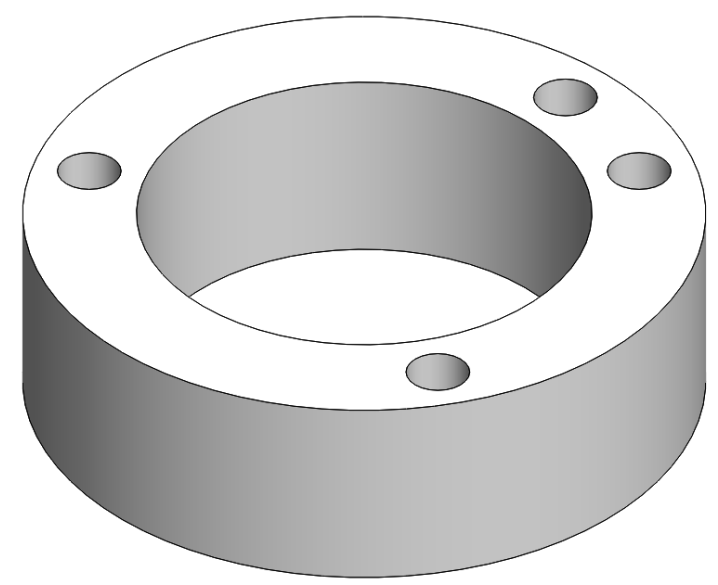

Figure 2.6: MiXI-CP-V3 Propellant Isolator Solid Model

\subsubsection{Cathode Keeper}

The keeper is a graphite hollow tube with an orifice on the top end and a flange on the base. This component is electrically charged and keeps the plasma and high energy electrons from returning to the propellant line and heater assembly. The keeper is $47 \mathrm{~mm}$ tall, has an outer diameter of $15.6 \mathrm{~mm}$, and has an inner diameter of $14.5 \mathrm{~mm}$. The orifice is $4.70 \mathrm{~mm}$ in diameter, and the top face is $1.27 \mathrm{~mm}$ thick. The flange at the base is $38.1 \mathrm{~mm}$ in diameter and $3.81 \mathrm{~mm}$ thick. There is an array of six through holes $4.83 \mathrm{~mm}$ in diameter centered on a circle of $31.75 \mathrm{~mm}$; these holes align with the through-holes in the propellant isolator and the cathode base. There is also a single threaded hole that connects to an all-thread power lead that is exposed through the cathode base. A solid model representation of the keeper is shown in Figure 2.7. 


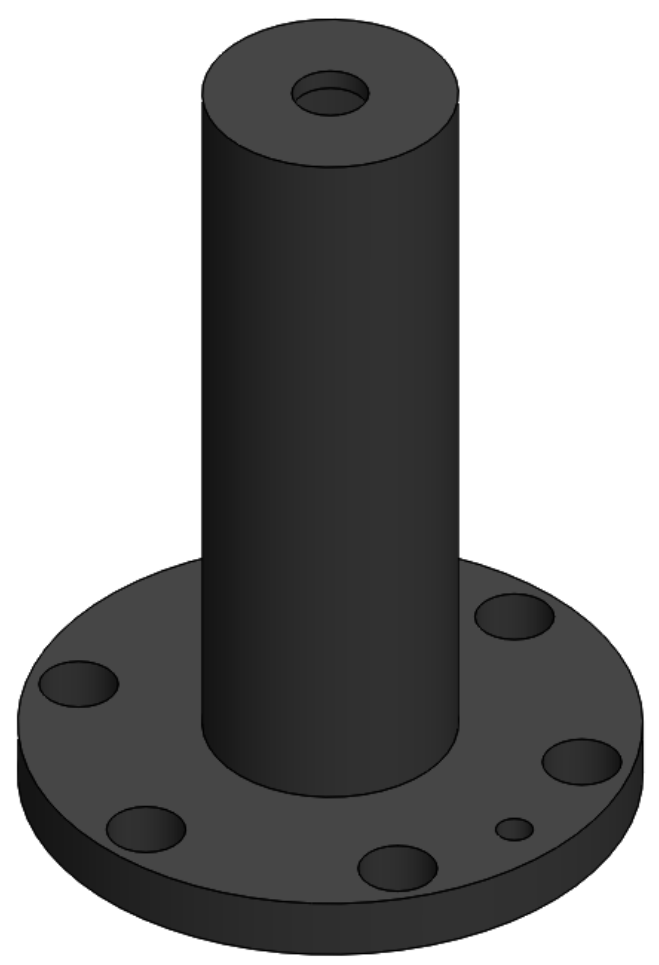

Figure 2.7: MiXI-CP-V3 Cathode Keeper Solid Model

\subsubsection{Keeper Isolator}

The keeper isolator is used to isolate the keeper from the chassis of the cathode. The keeper isolator is a ceramic ring $3.86 \mathrm{~mm}$ thick with an outer diameter of $38.1 \mathrm{~mm}$ and an inner diameter of $25.4 \mathrm{~mm}$. There is an array of eight through-holes $3.56 \mathrm{~mm}$ in diameter and centered on a circle of $31.75 \mathrm{~mm}$; these holes align with the through-holes in the keeper, propellant isolator, and the cathode base. A solid model representation of the keeper isolator is shown in Figure 2.8. 


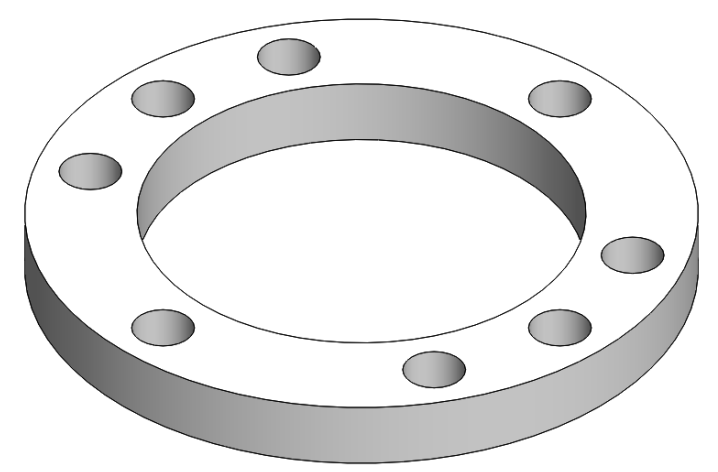

Figure 2.8: MiXI-CP-V3 Keeper Isolator Solid Model

\subsubsection{Cathode Chassis}

The cathode chassis is a stepped metal ring with a two arrays of holes and is used to attach and hold many of the components of the cathode assembly. The base flange is $63.5 \mathrm{~mm}$ in diameter and $3.18 \mathrm{~mm}$ thick. There is an array of eight $2.54 \mathrm{~mm}$ threaded holes that can be used to connect the chassis to the spacecraft or any mounting equipment within the test chamber. The holes are also used to connect the anode base of the thruster to the chassis, fixing the thruster to the cathode at a set distance.

The chassis has an inner diameter of $40.64 \mathrm{~mm}$, an outer diameter of $43.18 \mathrm{~mm}$, and is $18.85 \mathrm{~mm}$ tall overall. The upper flange has an inner diameter of $23.54 \mathrm{~mm}$ and is $2.67 \mathrm{~mm}$ thick. There is an array of six threaded holes in the upper flange centered on a $31.75 \mathrm{~mm}$ circle and provides the attachment point through the keeper isolator, the keeper, the propellant isolator, and into the propellant base. A solid model representation of the cathode chassis is shown in Figure 2.9. 


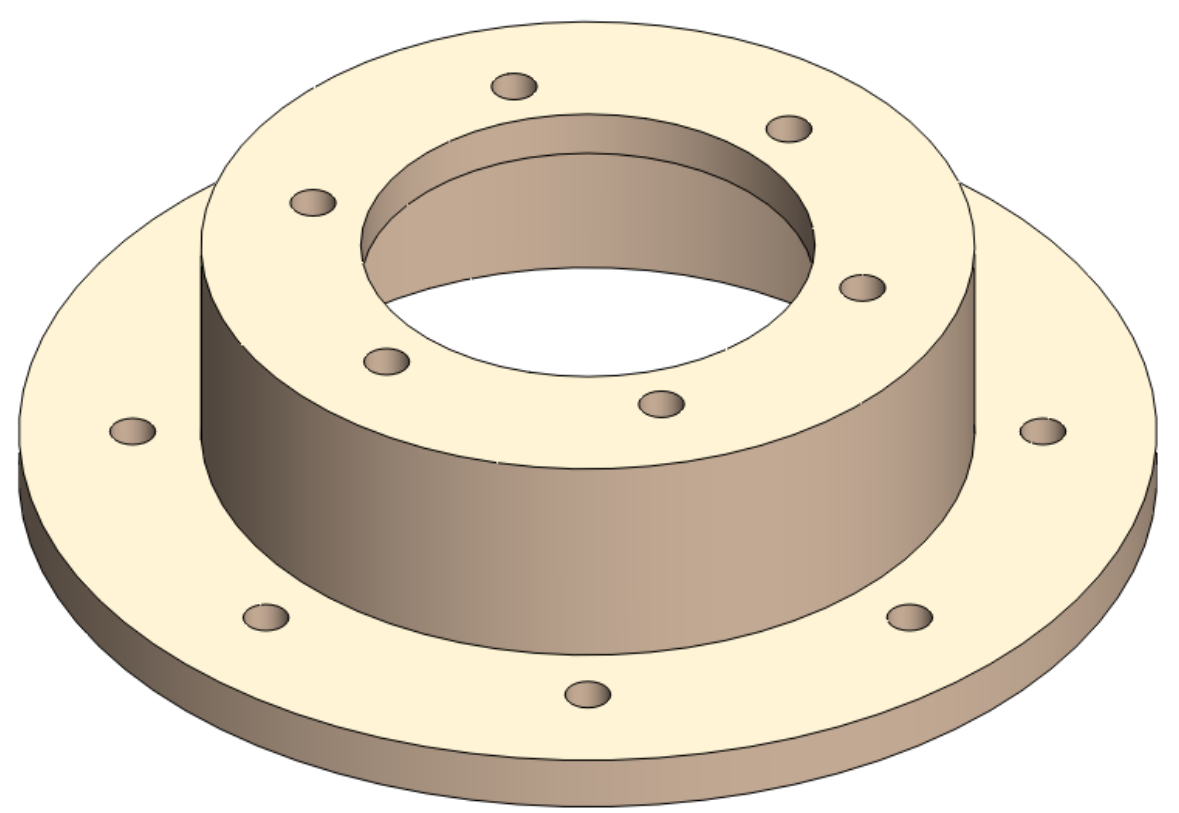

Figure 2.9: MiXI-CP-V3 Cathode Chassis Solid Model

\subsubsection{Keeper Isolation Sheath}

The keeper isolation sheath is a thin cylinder that encloses the end of the keeper and is intended to reduce the thermal flux from the end of the cathode directly to the base magnetic ring of the ion thruster to prevent overheating the magnets. The sheath also electrically isolates the keeper from the thruster base when fully assembled. The sheath is $10 \mathrm{~mm}$ tall and has an outer diameter of $18 \mathrm{~mm}$ and an inner diameter of $15.75 \mathrm{~mm}$. A solid model representation of the keeper isolation sheath is shown in Figure 2.10. 


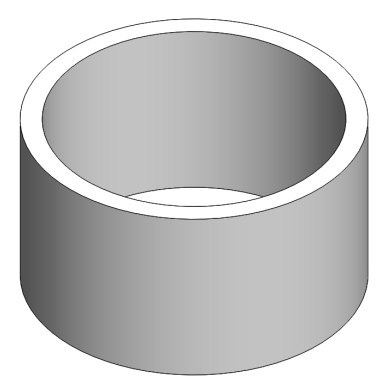

Figure 2.10: MiXI-CP-V3 Keeper Isolation Sheath Solid Model

\subsubsection{Hollow Cathode Assembly}

The components of the hollow cathode are stacked into the chassis and attached with allthread screws and nuts. Care must be taken when building the hollow cathode to keep the pieces electrically isolated from each other. Small ceramic isolators are used around all the screws and the heater line during final assembly to keep the chassis, keeper, and heater electrically isolated. The process for assembling the cathode is listed below.

1. Insert three all-thread screws equally spaced into the top flange of the chassis from the back end.

2. Slide the keeper isolator through the three screws until it is resting on the base of the upper flange.

3. Slide the keeper through the chassis opening and over the three screws as with the keeper isolator.

Ensure that the small threaded hole is resting on a solid ceramic space on the keeper isolator and not at a hole.

4. Attach the small keeper electrode all-thread screw to the base of the keeper.

5. Slide the propellant isolator over the now four screws and to the base of the keeper.

6. Attach the propellant line and associated heater to the cathode base.

Ensure the heater wire protrudes through the cathode base and is exposed near the Swagelok connector. 
7. Slide the propellant line and heater into the keeper and over the four screws until the propellant base contacts the propellant isolator.

8. Place four small ceramic isolators over the all-thread screws and into the cathode base.

9. Attach and tighten the appropriate hardware to the all-thread screws and the small ceramic isolators.

It is important not to over tighten the nuts on the screws as the small ceramic isolators and ceramic components within the assembly can crack. If any of the ceramic isolators break, they must be replaced.

An exploded view of the cathode assembly is shown in Figure 2.11.

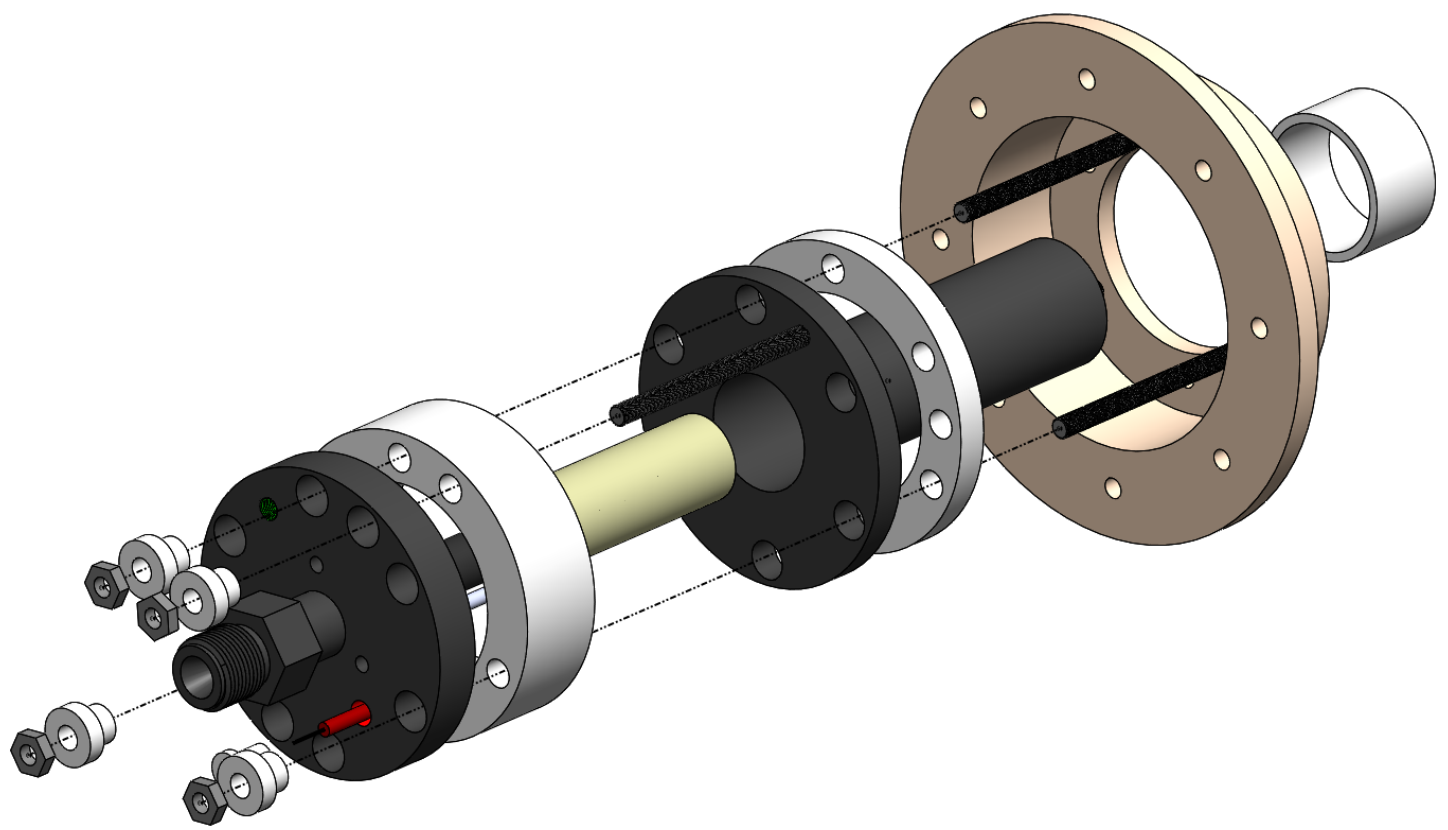

Figure 2.11: MiXI-CP-V3 Hollow Cathode Assembly, Exploded View showing all of the components of the cathode assembly and required attachment equipment

\subsection{MiXI-CP-V3 Design}

The MiXI-CP-V3 model varies from the baseline MiXI design developed by Wirz in order to accommodate the hollow cathode assembly. A cutaway view of the discharge chamber 
of the thruster and the installed components is shown in Figure 2.12. A detailed description of each part of the assembly are written in the following subsections.

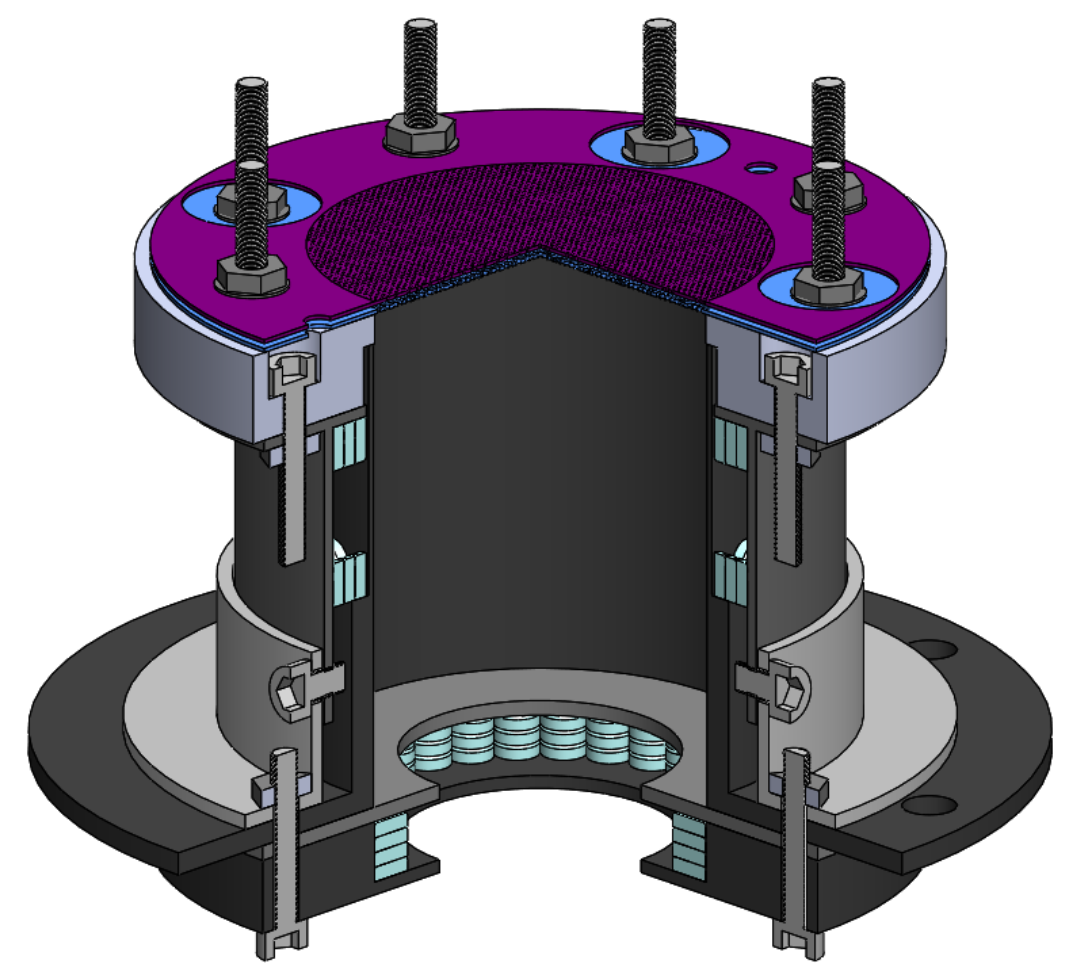

Figure 2.12: MiXI Discharge Chamber Fully Assembled Cutaway View

\subsubsection{Anode/Discharge Chamber}

The central component of the ion thruster is the discharge chamber. The ionization process and plasma generation takes place within, and the discharge current passes through, this component.

The anode has a $3 \mathrm{~cm}$ inner diameter and is $3 \mathrm{~cm}$ in length. This $1: 1$ ratio was discovered by Wirz to have the best performance when compared to different ratios of thrusters with this diameter [38]. The component is made of 303 stainless steel and is therefore nonmagnetic. The anode is connected to a power supply and biased positively relative to the 
cathode to help draw electrons out of the cathode and keeper while permanent magnets placed around the anode, visible in Figure 2.12, deflect the electrons away from the walls.

The anode is designed with a change in thickness: the top part of the anode where the magnets are located is $0.5 \mathrm{~mm}$ thick while the wall towards the base is $1.5 \mathrm{~mm}$ thick. The thin top portion allows for maximizing the magnetic field strength within the discharge chamber, while the thicker bottom wall increases the thermal volume of the part, drawing heat away from the magnets to postpone overheating and demagnetization $[19,30]$.

At the base of the anode is a larger flange that is the attachment point for many other thruster components. The flange is $66 \mathrm{~mm}$ in diameter and $2 \mathrm{~mm}$ thick. A solid model representation of the anode is shown in Figure 2.13.

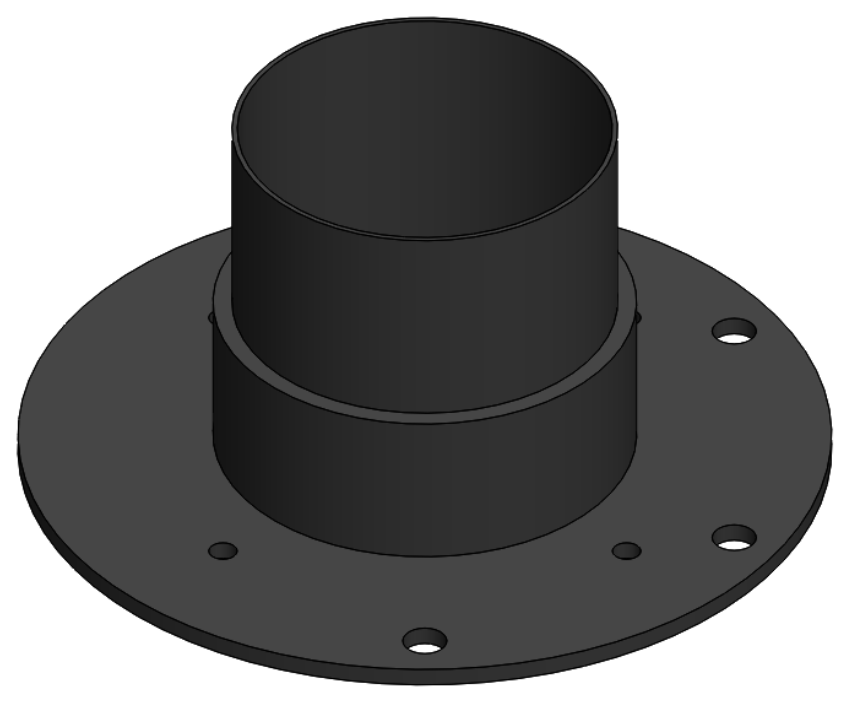

Figure 2.13: MiXI-CP-V3 Anode Solid Model

\subsubsection{Anode Magnet Mount}

The anode magnet mount is a magnetic steel component where the upper magnet ring cusps are attached. The mount is $2 \mathrm{~mm}$ tall, has an inner diameter of $37.54 \mathrm{~mm}$, and is $1 \mathrm{~mm}$ thick. The mount is made of magnetic steel which allows for the magnets to be attached without 
any fixtures or extra mounting hardware. The effect of the steel is analyzed in the Magnetic Model Section (3) of this report.

At the upper end of the mount is a $1 \mathrm{~mm}$ thick, $50 \mathrm{~mm}$ outer diameter, $31.5 \mathrm{~mm}$ inner diameter flange that is the attachment point for the screen and acceleration grids and the isolation mount. A solid model representation of the anode magnet mount is show in Figure 2.14 .

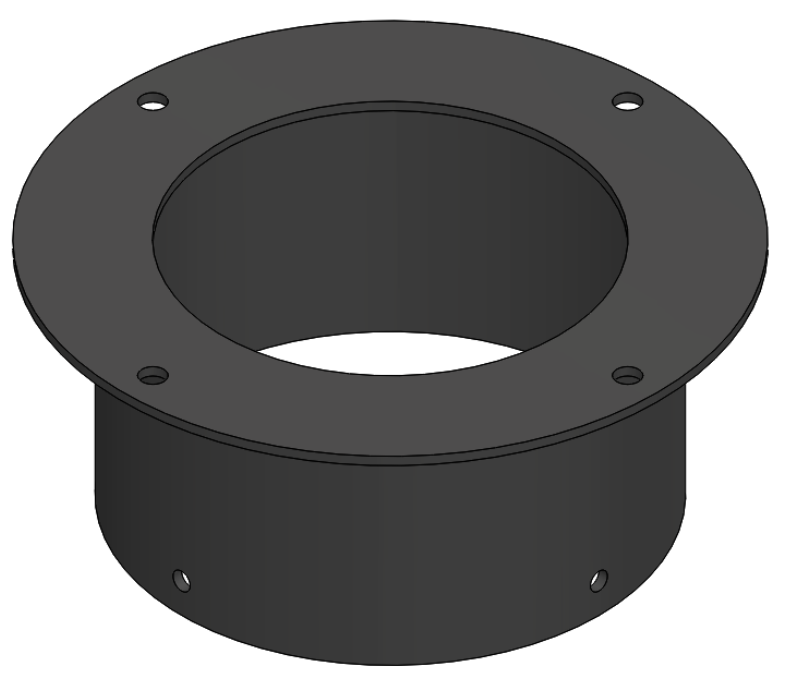

Figure 2.14: MiXI-CP-V3 Anode Magnet Mount Solid Model

\subsubsection{Anode Cap Piece}

The anode cap piece is a bracket that connects the wall of the magnet mount to the flange at the base of the anode. The cap piece is made of 303 stainless steel and is $10.52 \mathrm{~mm}$ tall. The cap piece wall is $1 \mathrm{~mm}$ thick and has an inner diameter of $39.45 \mathrm{~mm}$. The flange has an outer diameter of $53.45 \mathrm{~mm}$ and is $1 \mathrm{~mm}$ thick. A solid model representation of the cap piece is show in Figure 2.15. 


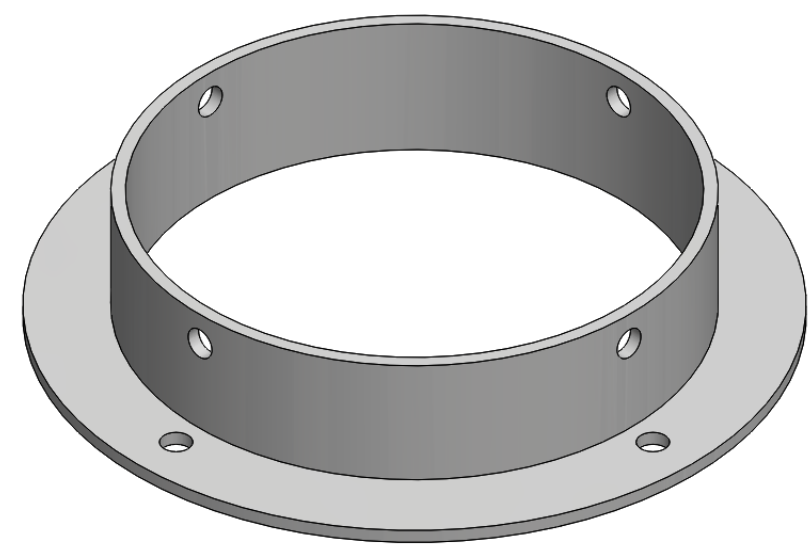

Figure 2.15: MiXI-CP-V3 Anode Cap Piece Solid Model

\subsubsection{Base Magnet Mount}

Similar to the anode magnet mount, the base magnet mount is a magnetic steel component designed to hold the base ring of the plasma confinement magnets. The base magnet mount is a disk with a $50.26 \mathrm{~mm}$ outer diameter and a $18.26 \mathrm{~mm}$ inner diameter and is $5 \mathrm{~mm}$ thick. There is a $4.12 \mathrm{~mm}$ deep cut with an outer diameter of $30 \mathrm{~mm}$ that provides a channel to house the lower ring of the confinement magnets. A solid model representation of the base magnet mount is show in Figure 2.16. 


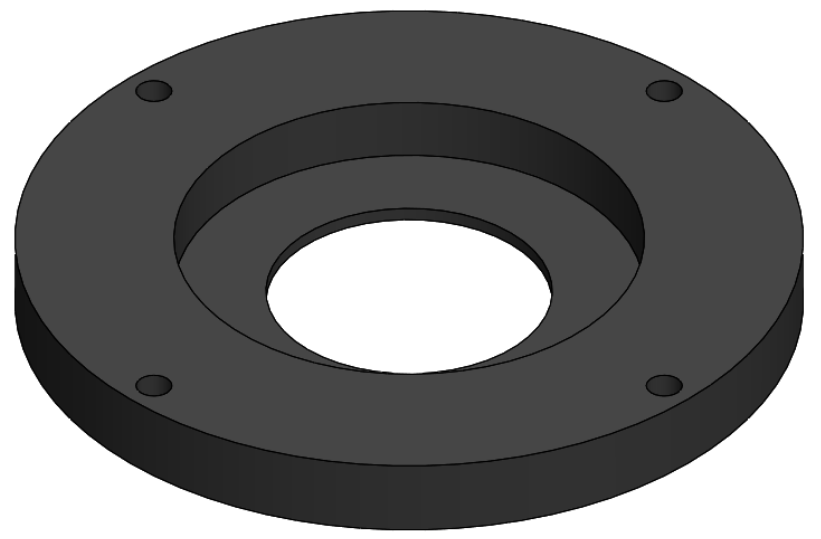

Figure 2.16: MiXI-CP-V3 Base Magnet Mount Solid Model

\subsubsection{Base Magnet Shield}

The base magnet shield is a disk that covers the magnet cavity and provides an insulating layer between the base magnetic ring and the anode base, providing the back face of the discharge chamber. The disk is $1 \mathrm{~mm}$ thick and matches the $50.26 \mathrm{~mm}$ outer and $16.26 \mathrm{~mm}$ inner diameters of the base magnet mount. The shield is made of non-magnetic 303 stainless steel so as to not attenuate or attract the confinement magnets. A solid model representation of the base magnet shield is show in Figure 2.17. 


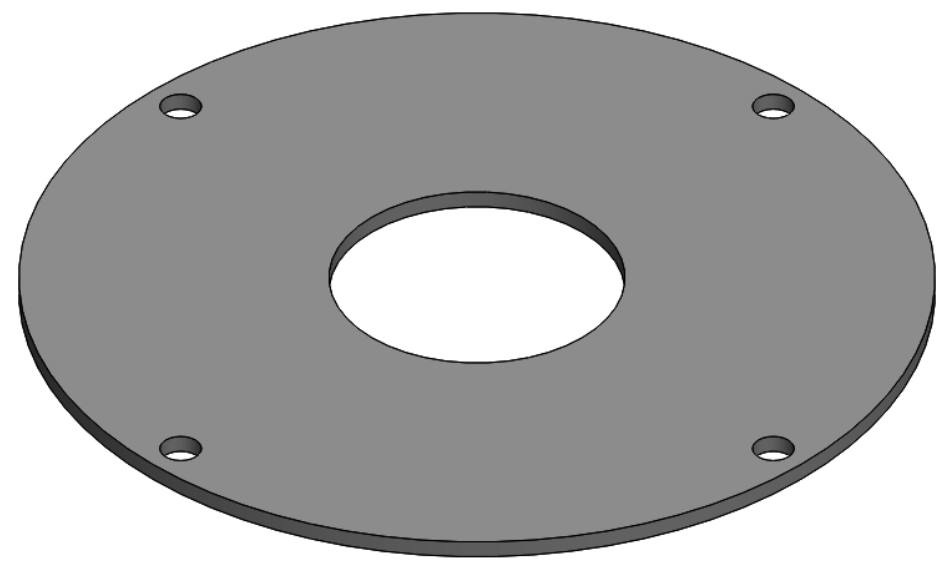

Figure 2.17: MiXI-CP-V3 Base Magnet Shield Solid Model

\subsubsection{Grid Isolation Mount}

The grid isolation mount electrically isolates and provides a mounting fixture for the screen and acceleration grids. The grid isolation mount is made from Macor, a machinable ceramic, for strength and electrical isolation.

The isolation mount has a $52 \mathrm{~mm}$ outer diameter, a $30 \mathrm{~mm}$ inner diameter, and is $6 \mathrm{~mm}$ tall. There is a step cut in the mount with an outer diameter of $32 \mathrm{~mm}$ and is $4 \mathrm{~mm}$ deep, allowing the anode to rest inside the isolation mount. There are two countersunk $2 \mathrm{~mm}$ through-hole patterns in the isolation mount: a series of four along a $44.5 \mathrm{~mm}$ diameter circle for attaching the isolation mount to the anode magnet mount shield, and a series of eight along a $40 \mathrm{~mm}$ circle to attach the screen and acceleration grids. The countersunk holes on both sides of the grid isolation mount allow for seamless attachment to the anode and with the grids.

A solid model representation of the grid isolation mount is shown in Figure 2.18. 


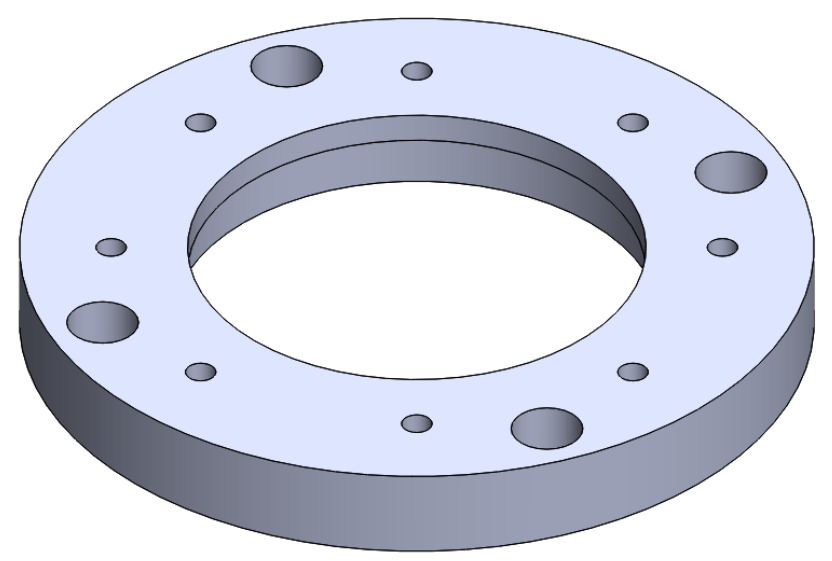

Figure 2.18: MiXI-CP-V3 Grid Isolation Mount Solid Model

\subsubsection{Screen and Acceleration Grids}

Grid design and sizing is an important aspect of and ion thruster's design. Proper understanding of ion optics and conductance reduction is important for creating an efficient thruster $[12,41]$. The screen and acceleration grids were created at JPL and donated for use in developing MiXI-CP-V1 [42]. The grids are made from molybdenum and are both $50 \mathrm{~mm}$ in diameter and $0.27 \mathrm{~mm}$ thick. Each grid has a centrally located circular area $30 \mathrm{~mm}$ in diameter where a total of 892 apertures are located. The screen grid, closest to the discharge chamber, has holes $0.75 \mathrm{~mm}$ in diameter while the acceleration grid, exterior grid, has holes $0.19 \mathrm{~mm}$ in diameter.

The grids used in MiXI are shown in Figures 2.19a and 2.19b. The screen grid, with the larger holes, can be seen on the left; the acceleration grid, with smaller holes, can be seen to the right. 


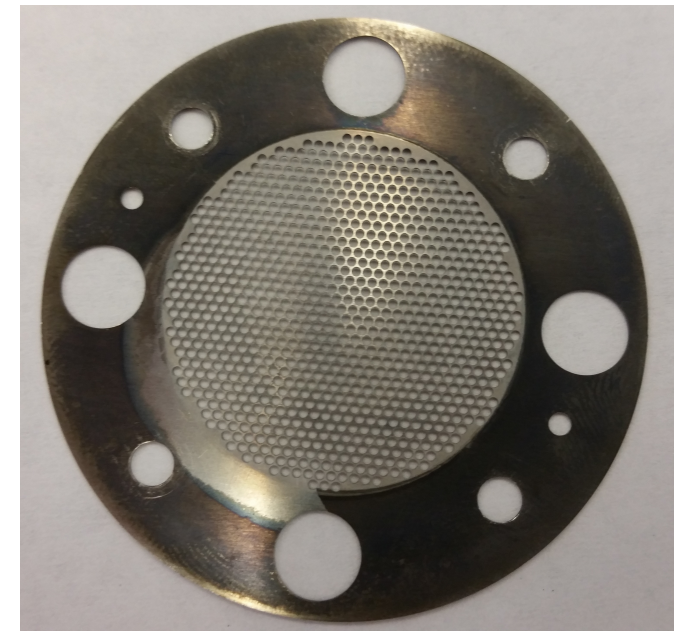

(a) MiXI Screen Grid

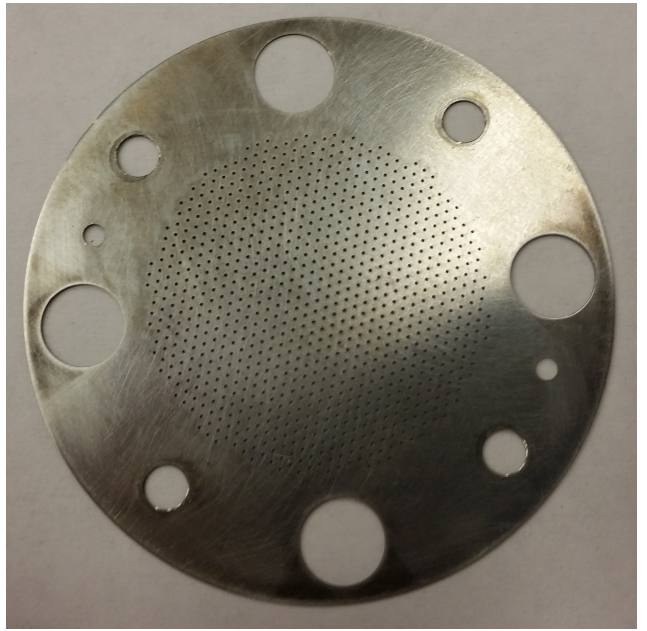

(b) MiXI Acceleration Grid

Figure 2.19: MiXI Screen and Acceleration Grids

The screen and acceleration grids were originally designed to have a gap of $0.3 \mathrm{~mm}$ between them. Over time, the grids have warped slightly and the original spacing leaves areas that are less than $0.3 \mathrm{~mm}$ in some places, leading to unwanted electrical breakdown and arcing between the grids. MiXI-CP-V3 was designed to have a gap of $0.6 \mathrm{~mm}$ between the grids. This is accomplished through the use of stacked $0.2 \mathrm{~mm}$ thick washers on the mounting screws. The number of washers can be varied to allow for a variable spacing depending on desired changes to the thruster. This spacing is illustrated in Figure 2.20. 


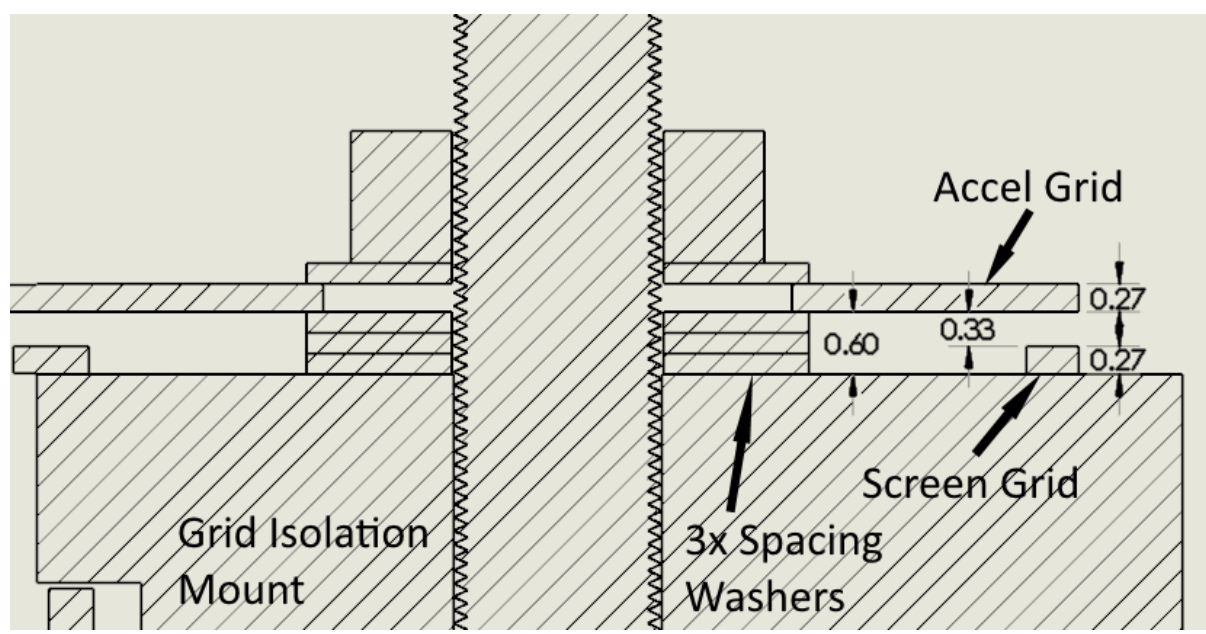

Figure 2.20: Spacing of Screen and Acceleration Grids achieved via spacing washers (All dimensions are in $\mathrm{mm}$ )

\subsubsection{MiXI-CP-V3 Assembly}

The assembly of the thruster takes place in two parts. There is a lower section consisting of the anode, the cap piece, the base magnet mount with associated magnets, and the base magnet shield. There is also an upper section consisting of the anode magnet mount and associated magnets, the grid isolation mount, and the screen and acceleration grids. The two halves attach between the anode cap piece and anode magnet mount when completed. The process for assembling the lower and upper sections are listed separately below.

Lower discharge chamber section assembly:

1. Install the magnets in the step of the base magnet mount.

Ensure the magnets are installed with the north pole up (towards the discharge chamber).

2. Stack the base magnet shield over the base magnet mount.

3. Stack the anode on top of the base magnet mount.

4. Stack the anode cap piece on the flange of the anode.

5. Insert the four screws through the holes that are aligned on this stack and tighten with the appropriate hardware. 
An exploded view of the assembly of the discharge chamber lower section is shown in Figure 2.21.

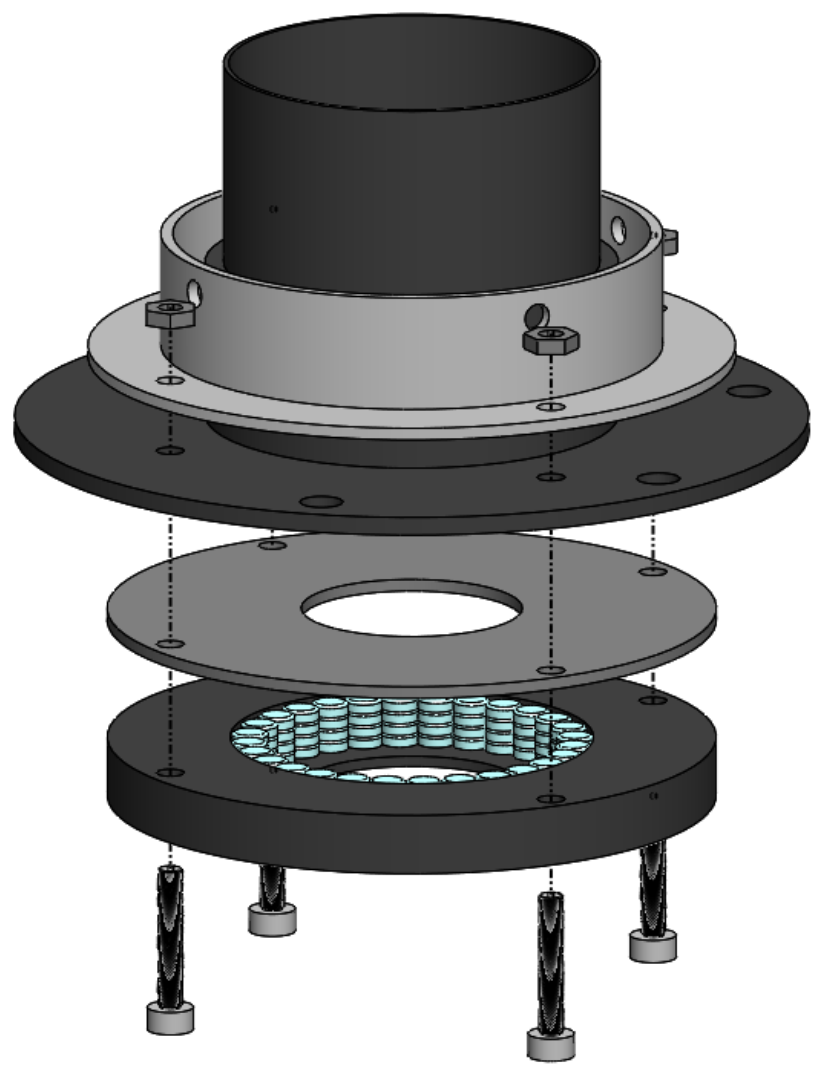

Figure 2.21: MiXI Discharge Chamber Lower Section Exploded View

Upper discharge chamber section assembly:

1. Install the two rings of magnets in the anode magnet mount.

First install the ring to the flange of the mount, the north poles of the magnets must be facing inwards towards the central axis.

Next install the ring in roughly the middle of the mount; the north poles of the magnets must be facing outwards. The magnets will be moved to their correct position when the two halves are attached.

2. Place twelve of the appropriate socket head screws in the countersunk sections of the grid isolation mount. 
3. Place the screen grid over the eight screws protruding from one side of the grid isolation mount.

4. Attach the screen grid with one washer and a nut. Tighten gently with a hex and Allen wrench, being careful not to damage the ceramic.

5. Place the appropriate number of washers over the four screws not being used by the screen grid hardware. These should rest directly on the ceramic isolator.

6. Place the acceleration grid over the eight protruding screws offset from the screen grid. The large clearance holes should lay over the hardware used to attach the screen grid.

Align the grids using the two pairs of holes manufactured in the grids.

7. Attach the acceleration grid with one washer and a nut. Tighten gently with a hex and Allen wrench, being careful not to damage the ceramic.

8. Place the isolation mount and grids on the flange of the anode magnet mount.

9. Attach the isolation mount and grids to the flange with a nut. Tighten gently with a hex wrench, being careful not to damage the isolation mount.

An exploded view of the assembly of the discharge chamber upper section is shown in Figure 2.22. 


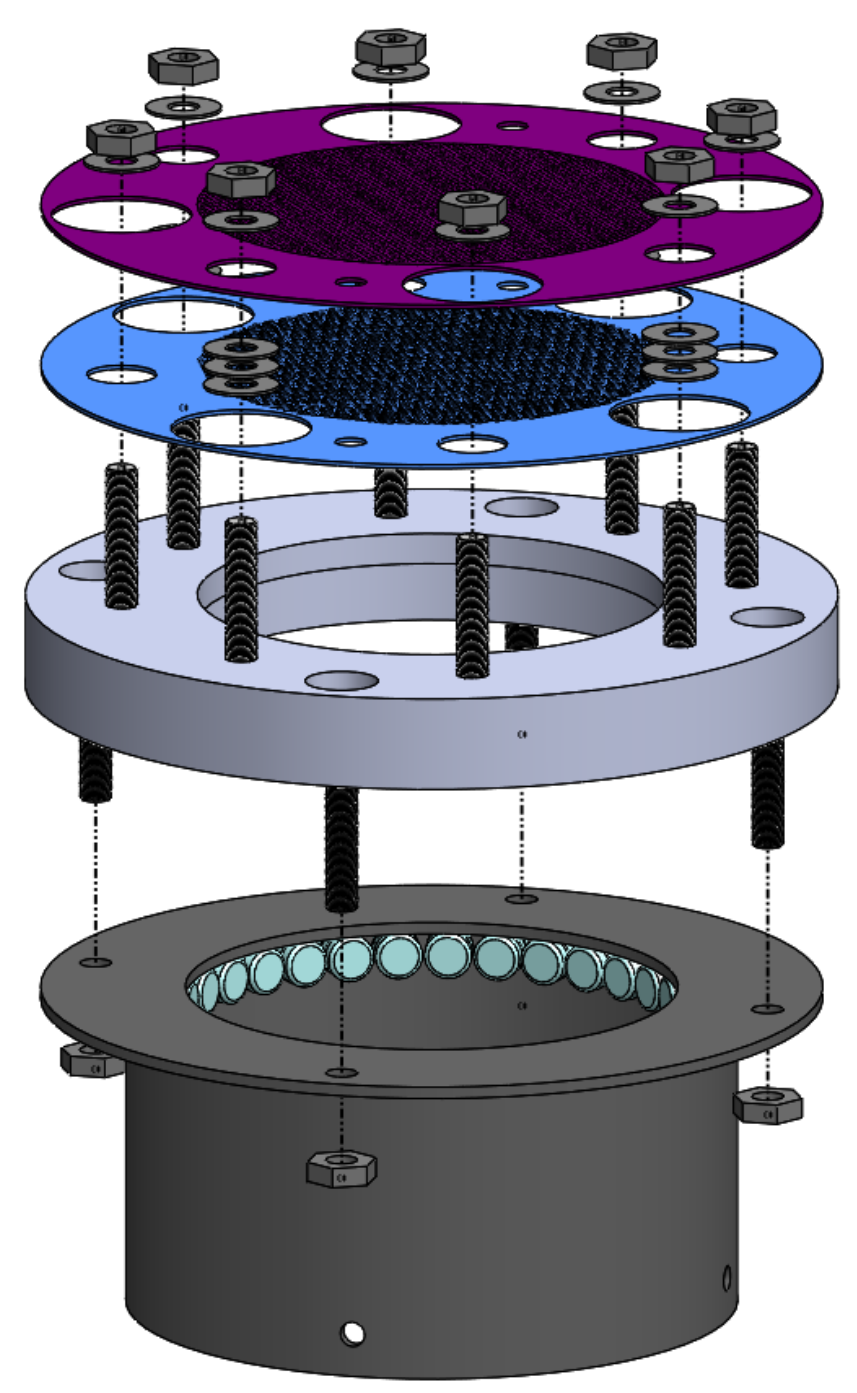

Figure 2.22: MiXI Discharge Chamber Upper Section Exploded View

The two halves of the thruster slide together with the anode magnet mount over the stepped anode and within the anode cap piece. The step on the anode will push and align the middle ring of the magnets installed in the anode magnet mount to their correct position the first time the thruster is connected. The four holes on the anode cap piece will align with the anode magnet mount and the appropriate hardware is used to attach the two halves.

A view of the full assembly of the discharge chamber is shown in Figure 2.23. 


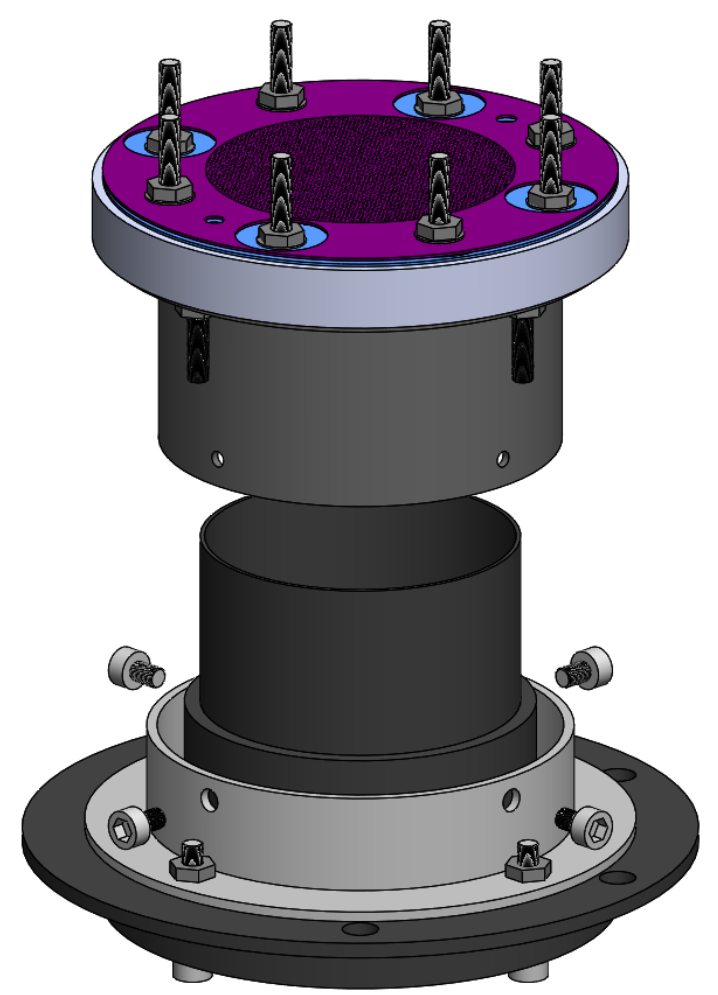

Figure 2.23: MiXI-CP-V3 Discharge Chamber Assembly

\subsection{Final Assembly}

With the hollow cathode and the thruster body assembled separately, the final assembly of the whole thruster is very straight forward. The hollow cathode and ceramic isolation sheath are inserted into the hole in the base of the discharge chamber assembly. The depth of the cathode inside the discharge chamber can be varied depending on test configuration. Different cathode assemblies for MiXI-CP-V3 may require different hardware to attach the hollow cathode to the truster; for testing in this thesis, the two assemblies were attached to an external chassis mounted in the test chamber.

The hollow cathode placement within the discharge chamber is highly dependent on the magnetic field strength at the orifice of the hollow cathode. The orifice must be placed at a location where the magnetic field is decreasing along the centerline of the cathode. 
This allows the plasma emitted from the orifice plate to diffuse smoothly into the discharge chamber and not be reflected back into the cathode [12].

The magnetic field within the chamber has already been determined for a given magnetic configuration and it is possible to use a program called FEMM (discussed in detail in Section 3) to determine the magnetic field strength within the chamber along the center axis. There were a number of magnetic field configurations considered; the configuration shown here was the baseline configuration most closely replicating previous theses. For much of this thesis, the orifice of the cathode was placed at a distance of $8 \mathrm{~mm}$ from the anode base within the discharge chamber.

The completed thruster has an overall length of about $11 \mathrm{~cm}$ and a maximum cross sectional area of about $33.75 \mathrm{~cm}^{2}$. The completed thruster (without any support hardware) is shown in Figure 2.24.

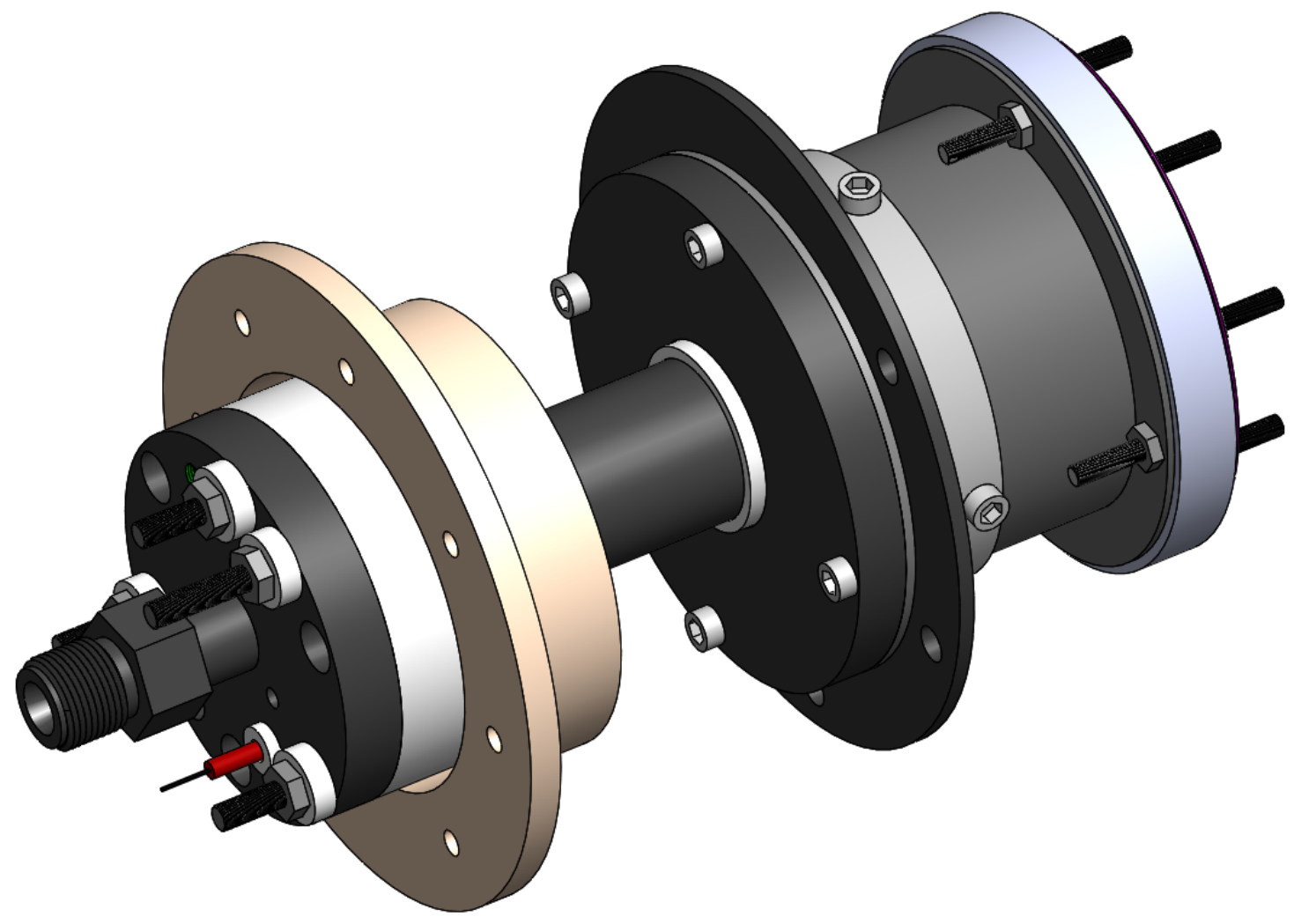

Figure 2.24: MiXI-CP-V3 Fully Assembled 
Chapter 3

\section{MAGNETIC MODEL CREATION AND ANALYSIS}

In his original work on MiXI, Dr. Wirz determined the optimum design for the magnetic fields for this thrusters uses permanent magnets in a multiple ring-cusp structure [38]. This ring-cusp design provides two distinct areas within the discharge chamber and plasma. The areas of high magnetic field strength near the cusps are intended improve electron confinement, increases the probability of electron collisions and ionization, and provides confinement of the ions from the anode wall through ambipolar effects. The low strength

diverging magnetic field in the center of the discharge chamber is designed to prevent the over-confinement the plasma and maintain a uniform ion beam density profile [22].

Figure 3.1 represents the magnetic field within the discharge chamber for the MiXICP-V3 thruster obtained at the start of this thesis [19]. 


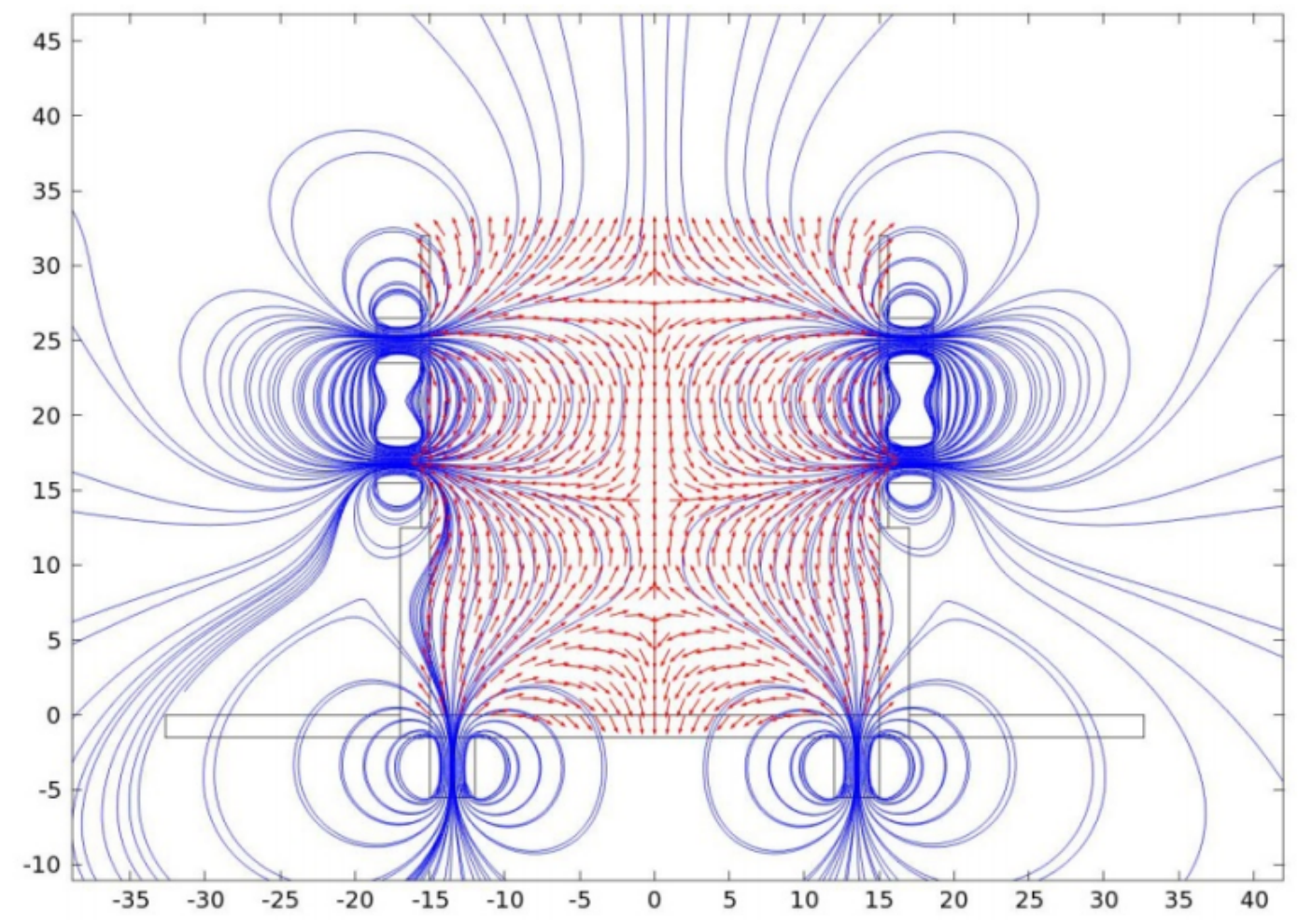

Figure 3.1: MiXI-CP-V3 Magnetic Field Model from Knapp's Thesis [19]

The blue lines represent the magnetic field streamlines while the red arrows show the magnetic field direction at the grid points. The higher density of blue field lines represents the convergence of the magnetic field where the permanent magnets are located. In this image, the three large gradient regions represent the location of the three magnetic ring cusps: one located at the base and two along the walls. The path of the streamlines give an indication of the orientation of the magnets.

The magnetic field generated within the discharge chamber is created by permanent magnets. As shown in the Computational Model Section (4), there are a few characteristics directly related to the design and analysis of the magnetic field, specifically the electron confinement factor, ion loss area, and hybrid loss area. The magnetic field design also has an effect on the plasma uniformity, the ion current density profile [14, 39], and electron emission stability from the cathode [12]. It is desirable to have a uniform, field free region 
in the center of the discharge chamber, having a closed field loop of sufficient strength, while also having a decreasing centerline field strength and having proper cusp strengths for electron confinement. The specifics of the different facets of the magnetic field design and their impacts are discussed further in section 3.3.

\subsection{Magnet Selection}

The initial selection and location of the permanent magnets for MiXI was determined previously by Knapp in his thesis and was derived from the work and conclusions from Wirz in his dissertation [38]. The end design from Kanpp was a three ring-cusp system with stacked Samarium-Cobalt grade 26 magnets. These small magnets are very heat resistant with a maximum operational temperature over $325 \operatorname{deg} C$ [21] and were determined to provide a high impact probability while maintaining a stable plasma potential $[19,12]$. The location of the magnets are constrained by the design of the anode and pole pieces constructed by Knapp. The upper confinement ring was positioned $24 \mathrm{~mm}$ axially from the anode base, the lower confinement ring $17 \mathrm{~mm}$ axially from the anode base, and the bottom confinement ring at the anode base but spaced $13.5 \mathrm{~mm}$ radially from the center. This setup was considered the baseline configuration of the thruster and referenced hereafter as

such. A cutaway view of the MiXI solid model with the magnets and their orientation and location is shown in Figure 3.2. 


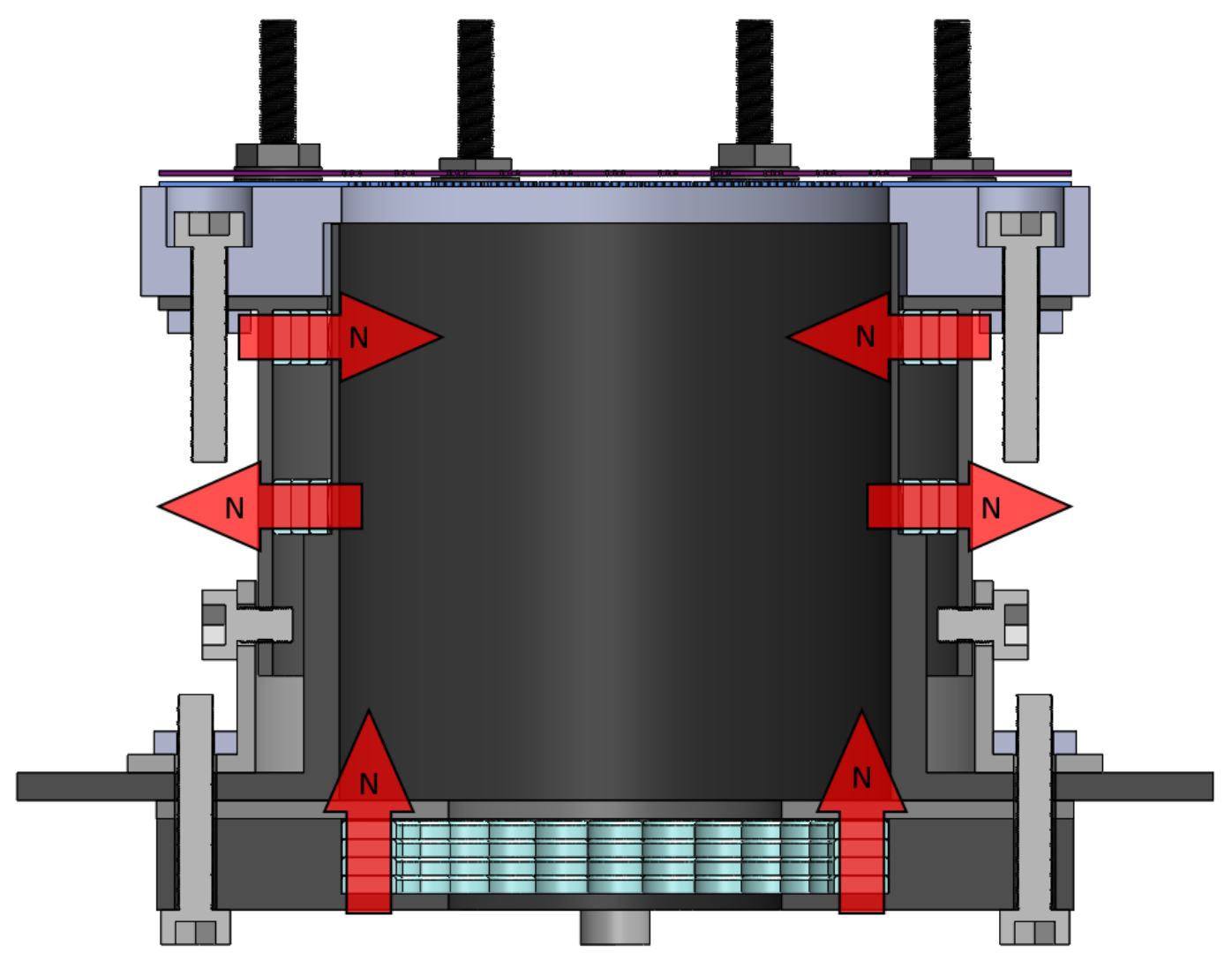

Figure 3.2: MiXI solid model cutaway view showing the magnet location and orientation, baseline configuration

\subsection{Magnetic Model Creation}

There were many ways the magnetic field could be developed and analyzed for the purposes of this thesis. In the most simple form, the magnetic potential, $\phi_{m}$, can be derived from Maxwell's equations and Laplace's equation [16] to yield

$$
\phi_{m}(r, \theta)=\sum_{k=0}^{\infty} \frac{m_{k}}{r^{k+1}} P_{k}(\cos \theta)
$$

The values in the summation arise from Legendre polynomials, with $k$ as the Legendre index. The value $m_{k}$ is the magnetic moments of the multi-pole expansion, $r$ is the radius from the magnet, and $P_{k}(\cos \theta)$ are the Legendre polynomial variables. This equation is 
able to be solved explicitly to determine the magnetic field for distance and angle from a magnetic dipole source.

$$
\bar{B}(r, \theta)=\frac{m_{1}}{r^{3}}[2 \cos (\theta) \hat{r}+\sin (\theta) \hat{\theta}]
$$

The value $m_{1}$ is the magnetic dipole moment. This value is unique to the source of the magnetic field and takes various forms depending on the source. For permanent magnets, $m_{1}$ becomes

$$
m_{1}=\frac{B_{R} V_{m}}{4 \pi}
$$

where $V_{m}$ is the volume of the magnet and $B_{R}$ is the magnetic perveance specification of the magnet, also known as the residual induction. The perveance is a value specific to the material and quality of the metal or ceramic.

\subsubsection{FEMM Overview}

Using real magnets and considering the interfering materials rather than just ideal dipoles means there are some variations between what the equations above describe and the true magnetic field inside the discharge chamber. For much of this thesis, the magnetic model will be solved by a program called Finite Element Method Magnetics (FEMM) [27]. FEMM was developed by Dr. David Meeker and is an open source analysis tool for solving electrostatics or magnetics problems. FEMM is capable of performing analysis on 2D geometry for planar or axisymmetric systems and on permanent or frequency dependent problems. FEMM was used in this thesis to solve steady state axisymmetric magnetics and electrostatics systems.

FEMM operates by creating an unstructured triangular mesh throughout a user generated geometry, applying material properties to all closed sections of the geometry, then solving the sets of equations required to obtain the solution. The structure of the geometry is obtained either by building a.$d x f$ file within FEMM or importing a.$d x f$ file from a solid modeling program such as SolidWorks or AutoCAD. A .dxf file is a simplistic way to 
represent structures using points and curves and is used to store the geometry of the micro ion thruster. The user then specifies all the material properties of the sections to create a complete representation of the physical parts within FEMM.

Specifying the materials of the closed sections also involves setting the electric and magnetic characteristics of the different pieces; every part must be detailed to obtain an accurate solution. FEMM has an internal materials library that includes many types of metals and permanent magnets and was adequate for the purposes of this thesis. The material properties of the anode, cathode, grids, etc. were all assigned within the model. The magnets and polarity direction are also specified and assigned to their representative blocks. A full representation of the axisymmetric model is shown in Figure 3.3. The labels and colors are to help provide a distinction between the different materials. 


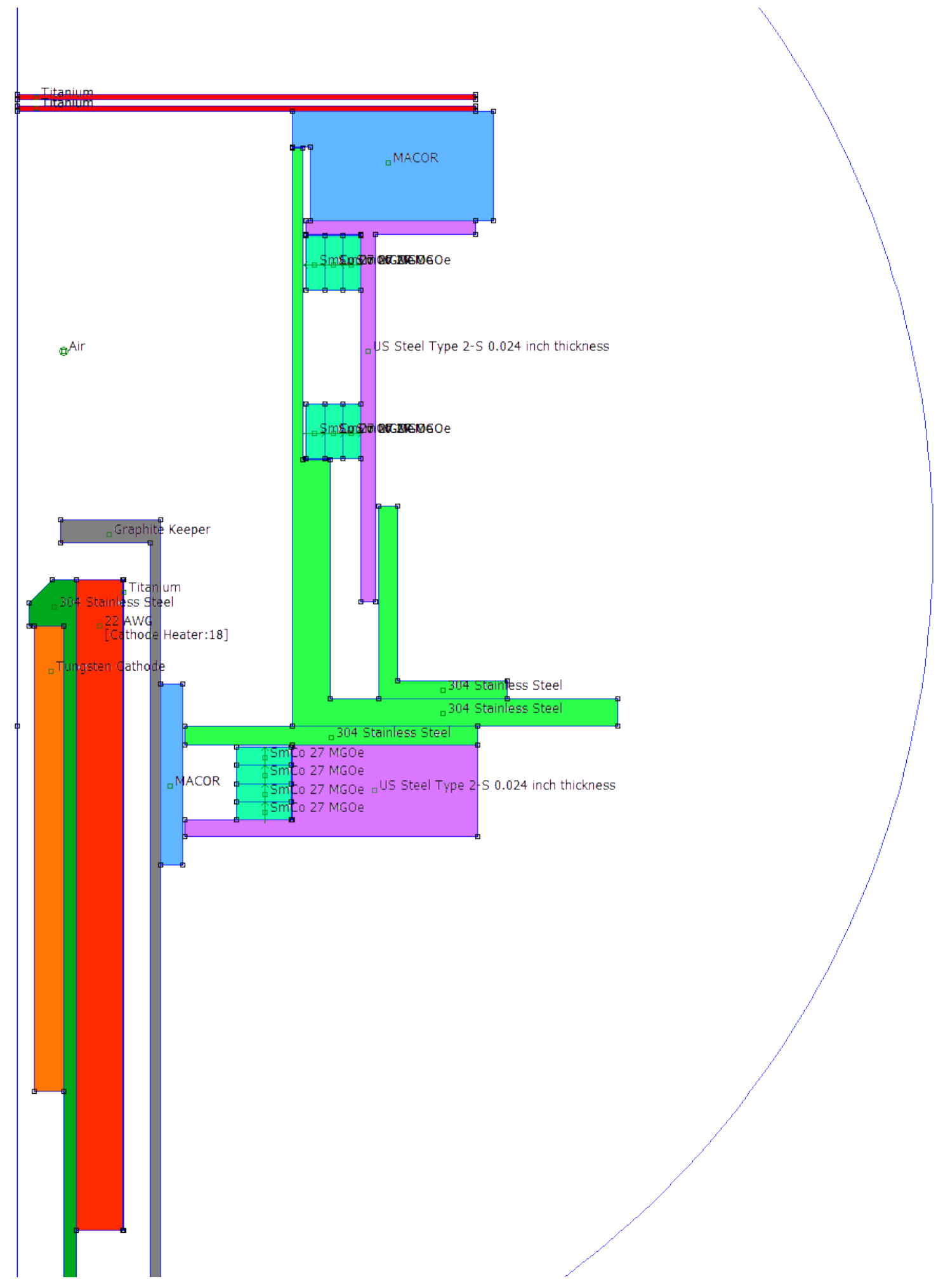

Figure 3.3: MiXI FEMM Model of the Thruster Structure Colorized and Annotated 
The anode wall and the main structure of the ion thruster is made of non magnetic stainless steel, represented in this image by the light green regions. The magnets, shown in cyan, are passively attached to the mangetic steel structure pieces, shown in purple. A machinable ceramic MACOR, shown in blue, is used to attach and insulate the charged anode from the charged grids, shown in red. A small MACOR sheath is used to isolate the cathode keeper from the rest of the thruster and is also shown in blue. The rest of the white space is empty vacuum. The blue arc around the edge represents the asymptotic boundary of the analyzed space. The left edge is the centerline of the thruster about which this axisymmetric model is built. A detailed description of the individual pieces of this thruster is contained in the System Configuration Section 2 of this report.

The power in this analysis lies in the fact that FEMM will account for the alterations in magnetic field through all the materials that make up the thruster. Also, with the model created in a modular fashion, it is easy to change the number and type of magnets that are built into the thruster. Different variations of the thruster were modeled and tested and building the model as shown made it simple to make changes that would not compromise any previous work.

\subsubsection{FEMM Magnetics Model}

A comprehensive magnetics model was constructed in FEMM that includes all the thruster parts and the permanent magnets contained within. The one electromagnetic source in this model is the heater which consists of a coiled wire around the tungsten cathode. Knowing the material and the amperage of the heater, it is possible to estimate and model the heater as a low power electromagnet at the base of the thruster. While insignificant by comparison, it was simple to include in the model and was done for completeness.

Once the geometry and material are specified for each piece, an unstructured mesh is created by FEMM and overlayed on the geometry. The "Air" node in the FEMM model was 
set to have an average mesh area of $0.35 \mathrm{~mm}^{2}$. A convergence study was performed and the methods and results are shown in Appendix E. The convergence study demonstrated that the mesh spacing used throughout the thesis has significantly small error and the results from FEMM are acceptable to use. The resulting mesh contained about 41348 nodes and 81925 elements; there are slight variations to these numbers based on the configurations generated. The mesh of the baseline configuration is shown in Figure 3.4.

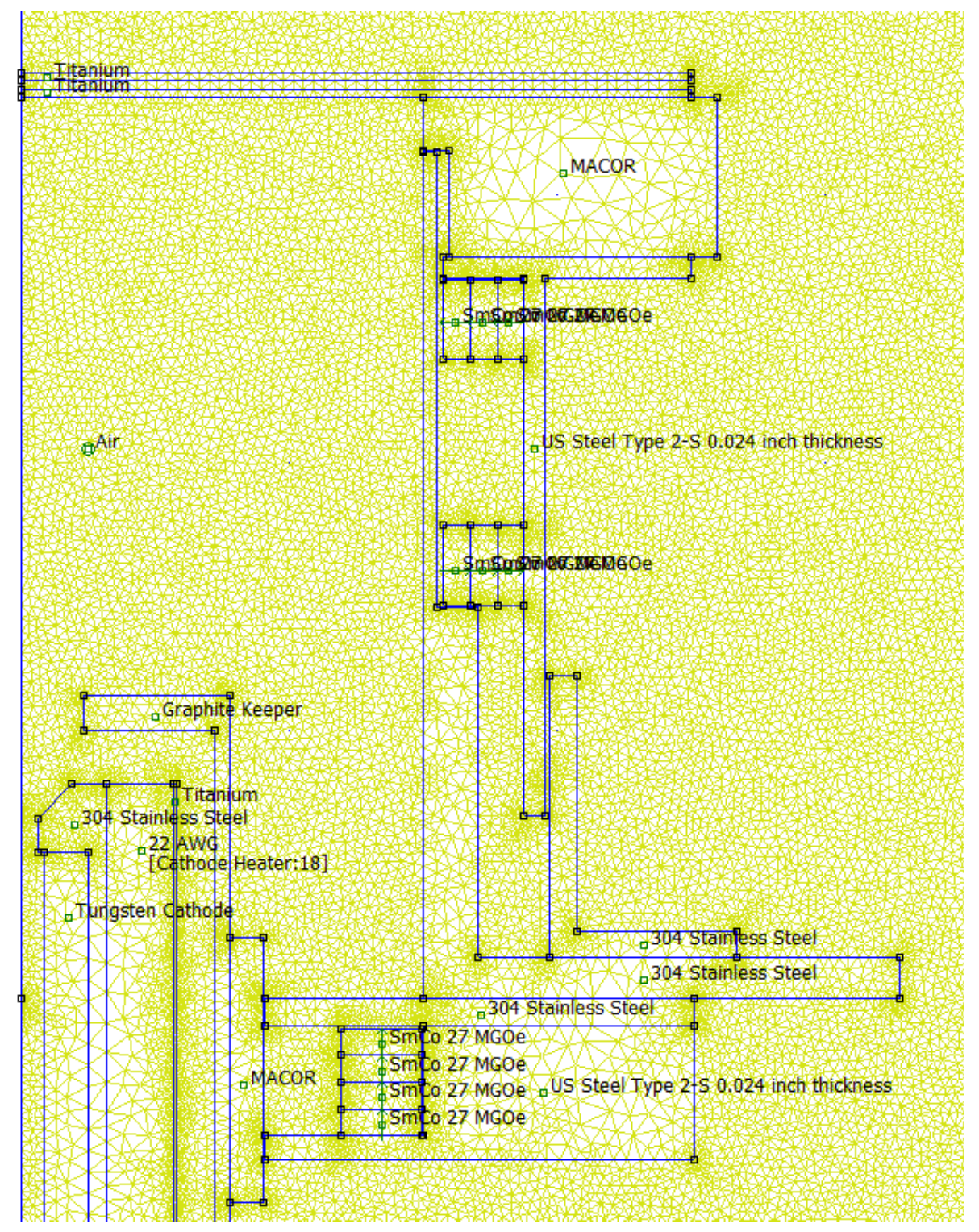

Figure 3.4: Baseline Configuration Mesh generated from FEMM 
With the mesh and geometry known, FEMM is able to solve for the magnetic field of the system. FEMM operates in a similar fashion to many CFD and other codes that operate on a mesh over a domain. FEMM solves Maxwell's equations while knowing the specific material permeability of each cell. FEMM finds a field that satisfies the equations via a magnetic vector potential approach. Complete equations and formulations of the problems can be found in the program documentation [27] or, more generally, in any Electricity and Magnetism textbook [16]. The magnetic solution produced by FEMM is shown in Figure 3.5 .

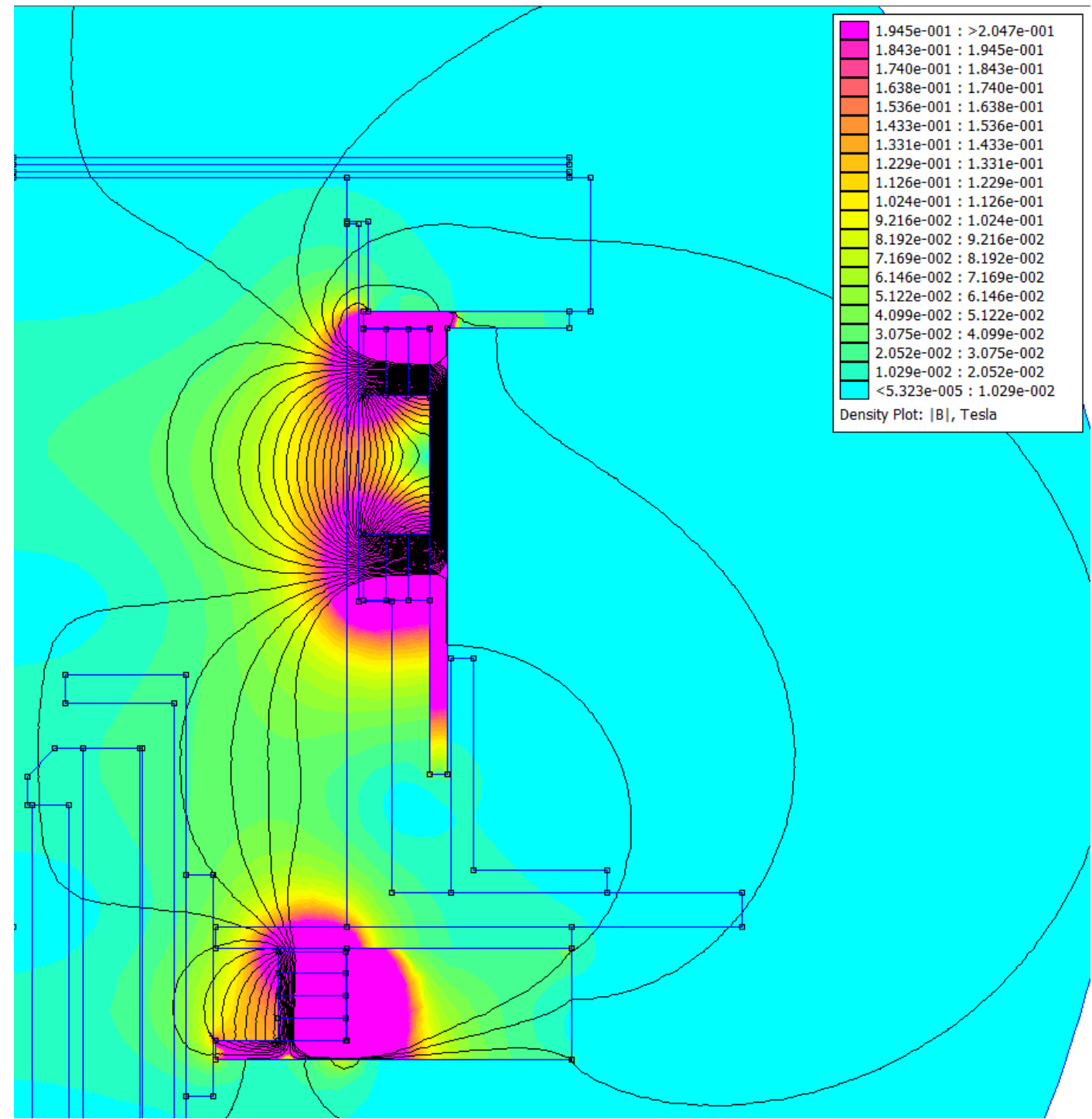

Figure 3.5: Magnetic Field Solution of the Baseline Configuration generated through FEMM 
There are slight variations in the solution shown in this model and the one presented previously in Figure 3.1. One reason is because the model generated for this thesis takes into account all the material properties of the individual components of the thruster. From Figure 3.5, it is very apparent the locations of the permanent magnets and the flux variations due to the ferrous metals serving as the magnet mounting surfaces. The color within the thruster and the walls represent the magnetic field strength while the solid lines depict the magnetic streamlines ${ }^{1}$.

While FEMM was a useful tool for generating the magnetic fields of the thruster, much of the post-processing for this thesis was performed in MATLAB. FEMM is capable of outputting data through the use of .lua scripts to .txt files. MATLAB is able to read the .txt files and perform the post-processing on the magnetic field. Figure 3.6 is an example of the magnetic field output from MATLAB.

${ }^{1}$ The material supporting the anode magnet rings is saturated; FEMM is capable of analyzing materials that are magnetically saturated [27]. 


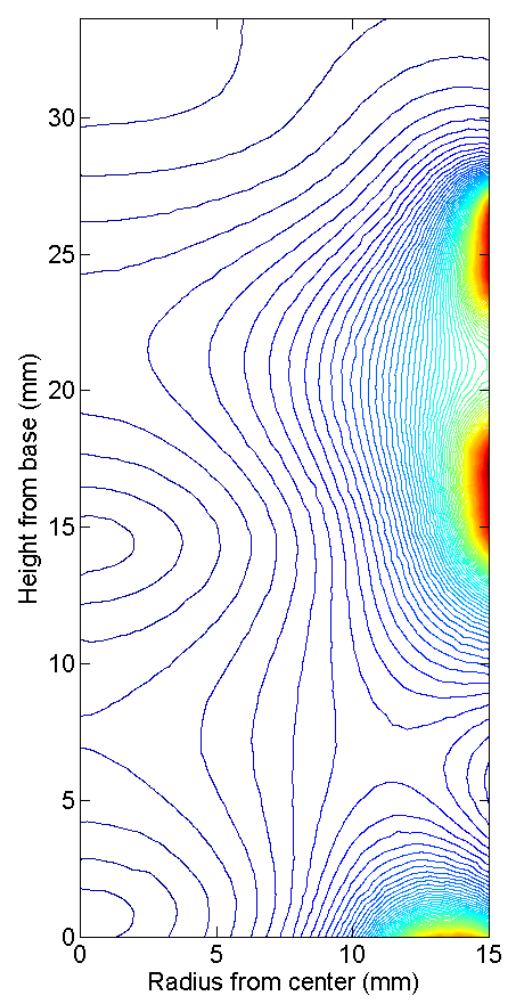

Figure 3.6: Magnetic Field Solution of Baseline Configuration displayed through MATLAB

\subsubsection{FEMM Electrostatic Model}

As with this magnetic model, an electrostatic model was build based on the same. $\mathrm{dxf}$ file and design geometry. In the electrostatic model, FEMM is able to assign the components and boundaries electric potential values that would be generated from the power supplies. FEMM solves for the electric field over the domain using a combination of Gauss's Law and Ampere's Loop Law while knowing the electrical permittivity of the materials. Again, the complete equations and formulations of the problems can be found in the program documentation [27]. It was possible to set the potential of the screen and acceleration grids, the keeper, the anode, and the cathode and analyze the electrostatics of the ion thruster. 
The same mesh generated for the magnetics model is used for the electrostatic model. The electrostatic solution of the thruster as solved by FEMM is displayed in Figures 3.7a and $3.7 \mathrm{~b}$. In this configuration, the screen grid was set to $500 \mathrm{~V}$ and the acceleration grid was set to $-100 \mathrm{~V}$ and set the bounds of the plot.

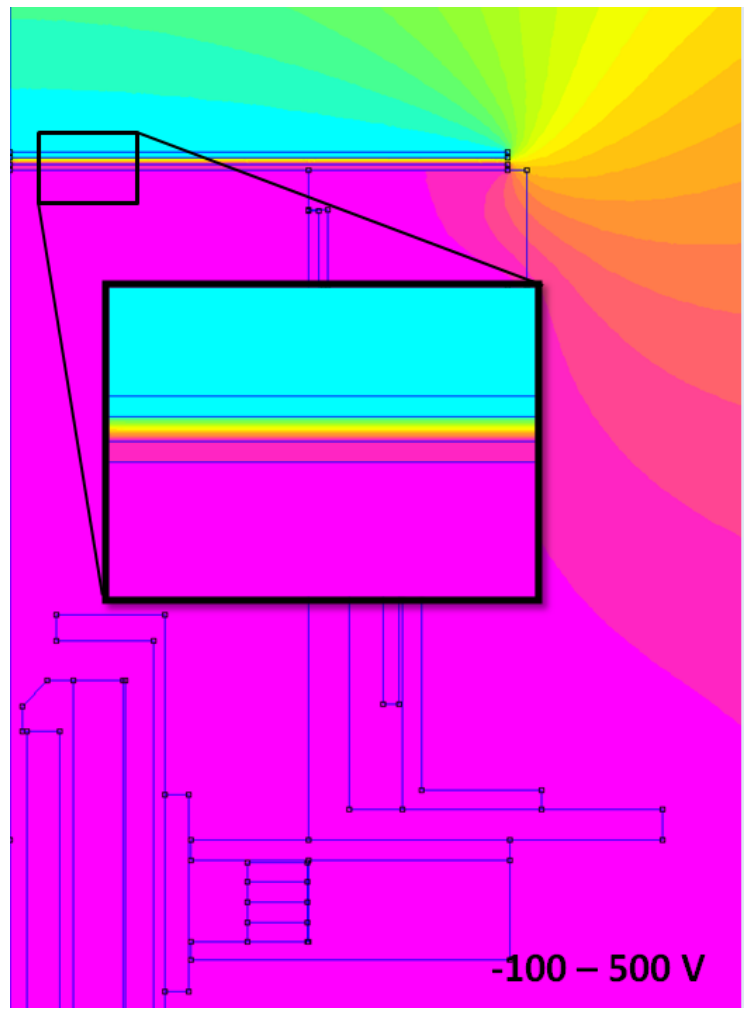

(a) Electric Field of Baseline Configuration generated in FEMM

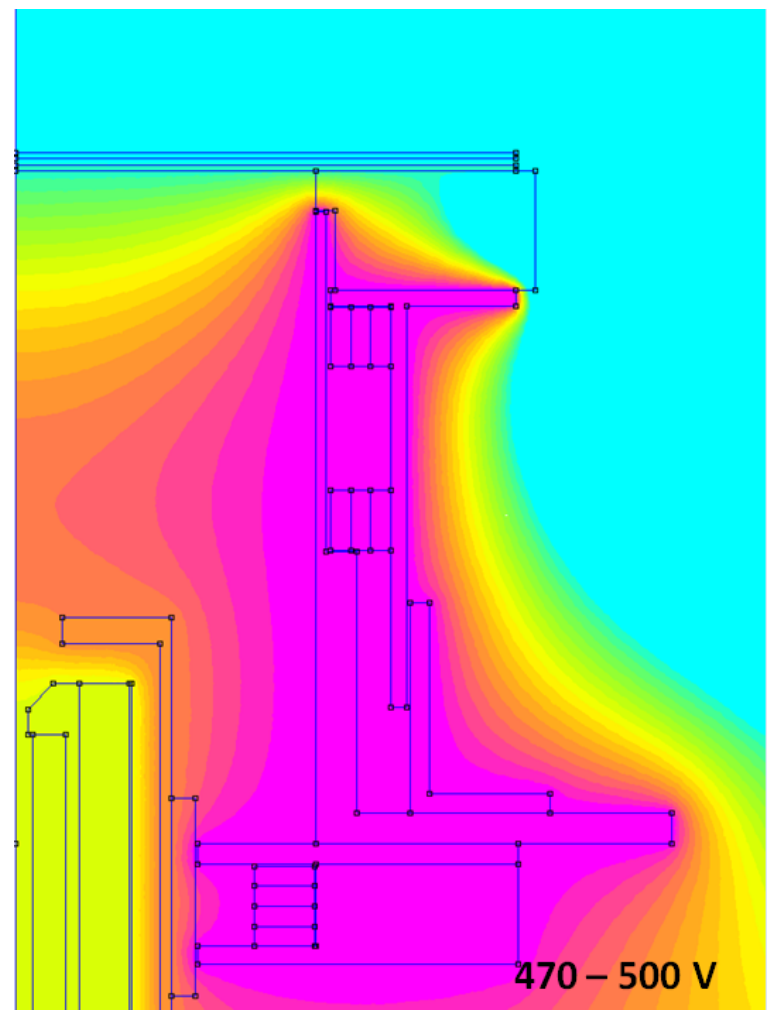

(b) Electric Field generated in FEMM, with voltage bounds

\section{Figure 3.7: Electric Field of the Baseline Configuration Generated in FEMM}

Figure 3.7a shows the solution with a zoomed box showing the high gradient field between the grids. Figure $3.7 \mathrm{~b}$ shows the electric potential bounded between $470 \mathrm{~V}$ and $500 \mathrm{~V}$ to show the effect of the anode components independent of the grid potentials. The purple regions represent the areas of high electric potential while the blue regions represent the areas of low electric potential in each figure. 
It is easy to see the effects of the high relative potential of the screen grid as opposed to the anode wall or cathode in the first of the two figures 3.7a. Inside the discharge chamber, the field is dominated by the anode wall, keeper, and screen, as shown in the second of the two figures $3.7 \mathrm{~b}$. The potential differences inside the chamber influence the flow of electrons and ions; once the ions pass the screen grid, their motion is dominated by the high gradient between the grids. Similarly with the magnetic fields, the electric fields were exported from FEMM and into MATLAB; the resultant figures are not shown here.

\subsection{Magnetic Field Analysis}

The proper analysis of the magnetic fields was critical to understanding the operation of the thruster. The magnetic field configuration has a direct effect on a number of important parameters, specifically, the confinement factor of the ions, the probability of primary electron collision, and the plasma potential. In order to understand the effect on these parameters, it is necessary to determine the field strength at the magnetic cusps and also calculate the closed loop field strength.

Determining the strength of the cusps is a simple matter of taking the magnitude of the field at the known magnet locations. For the baseline configuration, the top anode ring cusp has a magnetic field strength of 2920.98 Gauss, the bottom anode ring cusp has a strength of 3010.56 Gauss, and the base magnetic ring has a strength of 2206.95 Gauss. The cusp strengths have a direct impact on the primary electron confinement and the probability of collision between the high energy primary electrons and the neutral gas particles. It is desirable to have good confinement and high collision probability to increase the mass utilization of the injected fuel [12].

In his work, Wirz developed a particle in cell tracker code to analyze electron motion within a plasma thruster discharge chamber [38]. That code was used to analyze various thruster geometries and magnetic configurations, including MiXI. One such output is 
shown in Figure 3.8. This figure analyzed a discharge chamber similar to the NSTAR thruster and includes annotations about the electron and it's path.

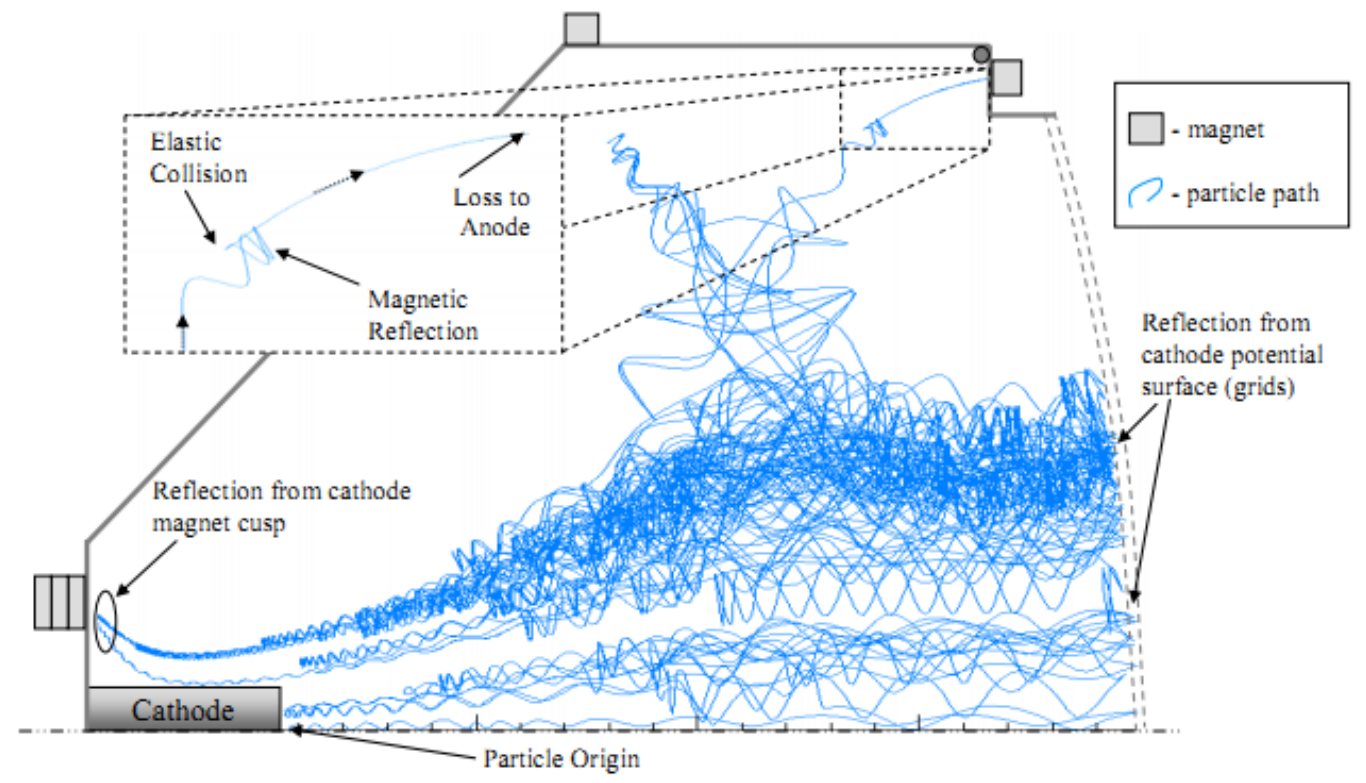

Figure 3.8: Electron Path in Plasma Discharge Chamber, NSTAR analog [38]

The magnetic field effects direct the electrons towards the magnetic cusps where they are either lost to the anode wall or undergo magnetic reflection. The probability of either is determined by the field strength at the cusps. A similar model was developed by Wirz which analyzes a geometry similar to MiXI and is shown in Figure 3.9. 


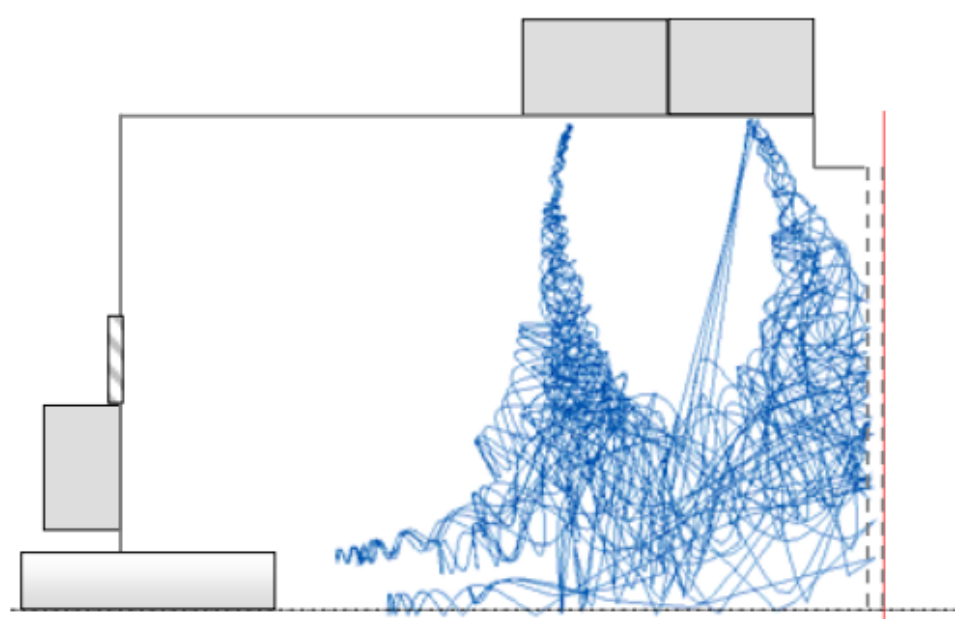

Figure 3.9: Electron Path in Plasma Discharge Chamber, MiXI Thruster [38]

To properly analyze the effects changing the cusps strengths will have on the collision probability, the full computational model developed for this thesis was used. The specifics and the supporting equations are all described in Section 4 and only referenced here.

\subsubsection{Primary Electron Collision}

The probability of collision is inversely exponentially proportional to the primary electron loss area $A_{p}$, shown in Equation 4.27. The primary electron loss area is inversely proportional to the magnetic field strength at the cusps and is shown in Equation 4.21. An analysis was performed changing the magnetic field strengths of all the cusps and determining the resultant probability of collision. The result of that analysis is shown in Figure 3.10. 


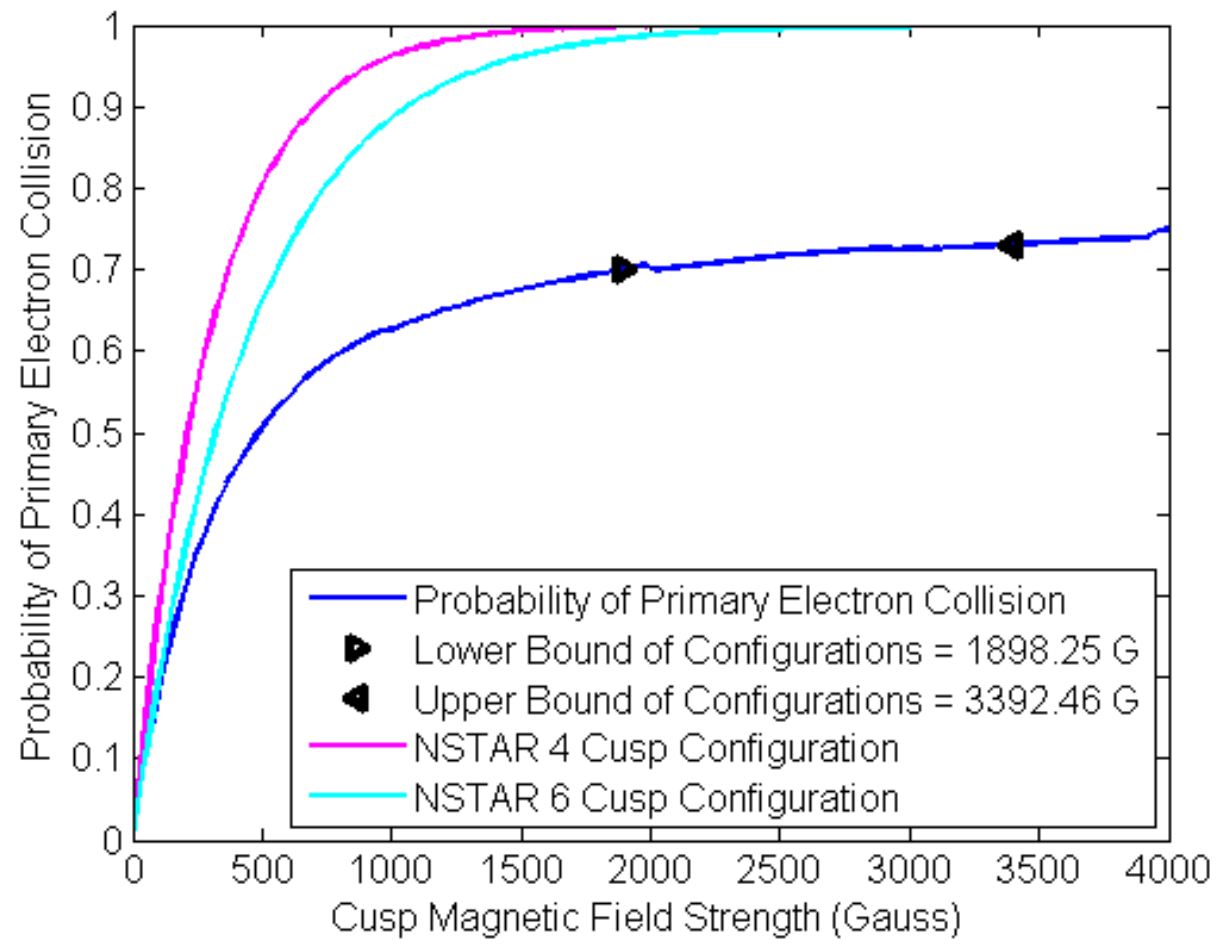

Figure 3.10: Probability of Primary Electron Collision with Changing Magnetic Field Strength

From the figure, it is easy to see the effect changing the magnetic field strength has on the collision probability. With an increased cusp strength, the magnetic reflection effect is greater, therefore the electrons are more confined and have a greater probability of impacting a neutral fuel particle. Also shown in this image are data sets from the NSTAR thruster. Most notable is the fact the collision probability is much greater for the NSTAR thruster than for MiXI. The main reason for this is the probability collision being exponentially proportional to the volume of the discharge chamber. MiXI has a much lower volume when compared to larger thrusters, greatly decreasing the collision probability.

The markers on the collision probability trace show the upper and lower bounds for all of the magnetic configurations analyzed in this thesis and described in Section 3.4. The lower bound magnetic field strength of the cusps is $1898.25 \mathrm{G}$ which corresponds to a collision probability of $70.12 \%$; the upper bound strength at the cusps is $3392.46 \mathrm{G}$ which 
corresponds to a probability of $73.09 \%$. While there is variation to the collision probability based on the design of the magnetic field, other terms dominate the effect of changing the cusp strength and keep the collision probability around $72.5 \%$.

\subsubsection{Plasma Potential}

The field strength at the cusps also has a direct impact on the plasma potential within the discharge chamber and the stability of the plasma discharge [15]. The plasma potential $\phi$ is directly proportional to the hybrid electron-ion loss area $A_{h}$ and is described in Equation 4.55. The hybrid loss area is inversely proportional to the field strength at the cusps and is shown in Equation 4.22. If the hybrid loss area becomes too small relative to the total surface area of the anode, the plasma potential will become negative relative to the anode potential. In this case, the plasma creates an "electron accelerating" anode sheath, and the anode area at the cusps is no longer the sole source of discharge current collection. An illustration of the various potentials of the discharge chamber and the decrease in primary electron energy is shown in Figure 3.11.

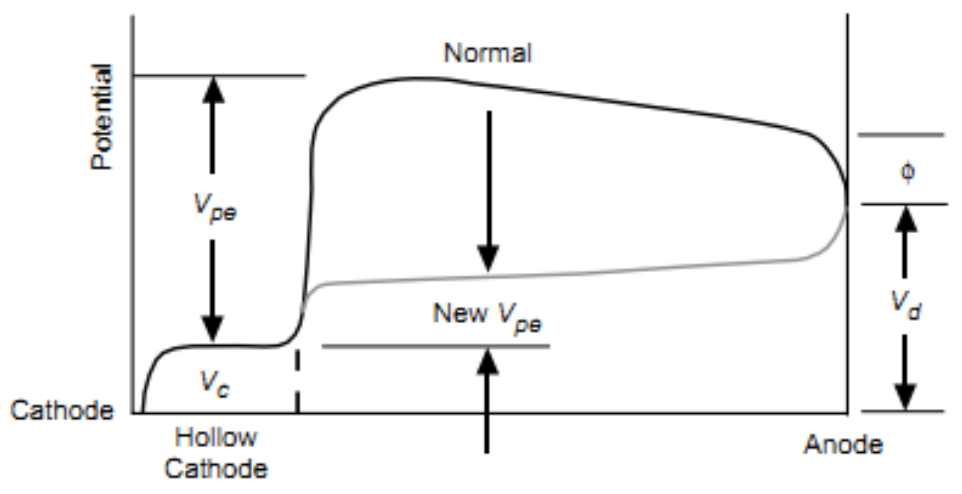

Figure 3.11: Plasma Potential Transition to a negative value with respect to the anode, resulting in lower primary electron energy [12]

To maintain higher energy primary electrons and stability of the plasma, it is desirable to keep a positive plasma potential within the discharge chamber. The effect of changing the field strength at the cusps is shown in Figure 3.12. 


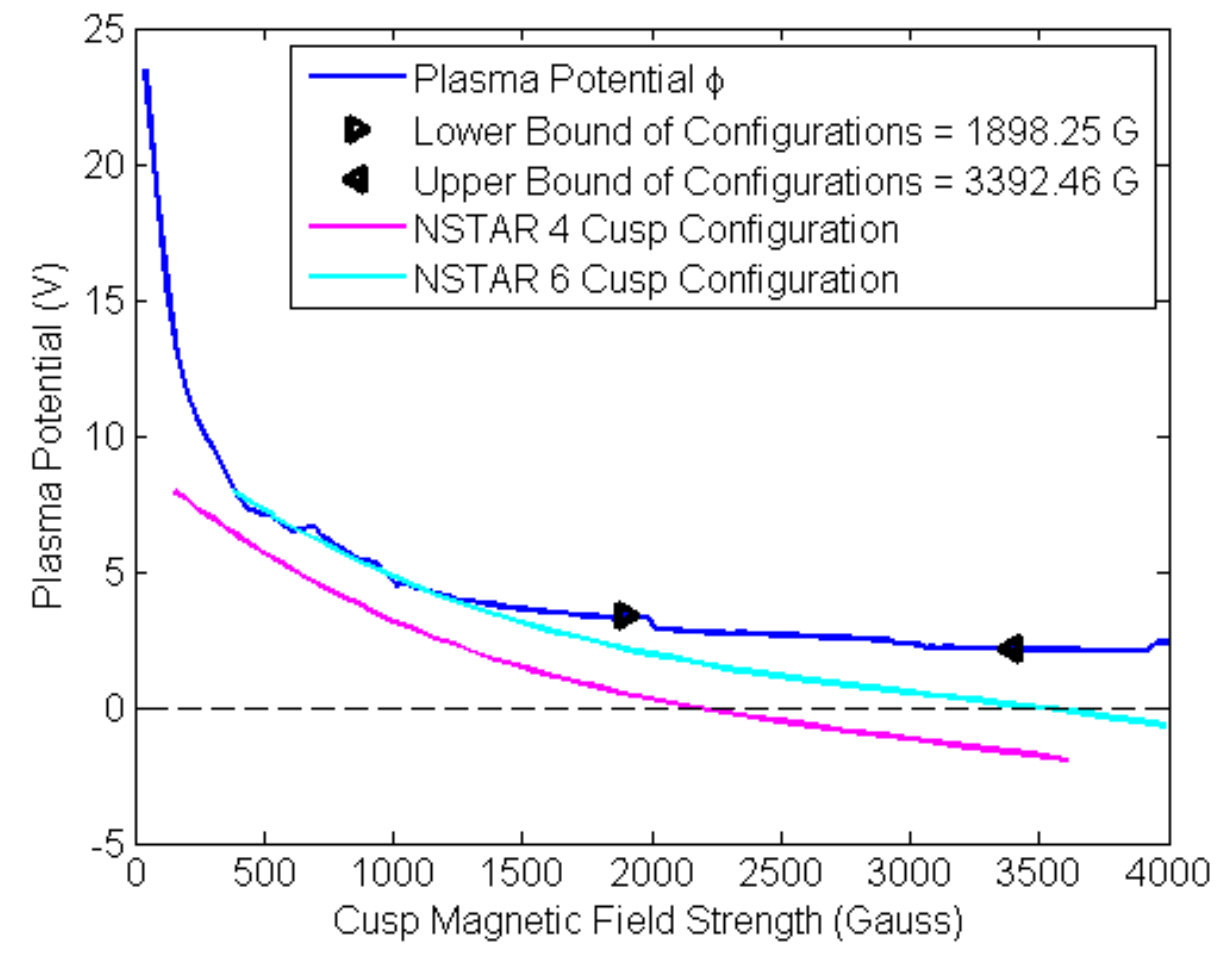

Figure 3.12: Plasma Potential with Changing Magnetic Field Strength

From the figure, it is easy to see the effect changing the magnetic field strength has on the plasma potential. The potential drops as the cusp field strength increases, leveling off at a value above zero. Also shown in this image are the plasma potential values from the NSTAR thruster with both configurations. Both NSTAR traces show the plasma potential drops to a negative value as the magnetic field strength of the cusps increases.

The markers on the plasma potential trace show the upper and lower bounds for all the magnetic configurations analyzed in this thesis and described in Section 3.4. The lower bound corresponds to a plasma potential of $3.35 \mathrm{~V}$, while the upper bound corresponds to a plasma potential of $2.15 \mathrm{~V}$. While there is variation in the plasma potential over the whole sweep, plasma potential is rather constant through the range of cusp strengths for the analyzed configurations. 


\subsubsection{Ion Confinement}

Another important value about the magnetic field design is the closed loop magnetic field strength within the discharge chamber. The closed loop strength directly affects the ion confinement at the anode, illustrated by the fraction of the ion current transverse to the magnetic field $I_{i a}$ to the Bohm current $I_{B o h m}$ and is represented by a confinement factor value $f_{c}$. Ambipolar effects within the closed loop magnetic field repel the ions from the anode walls and reduce the amount of ion current that is lost [12].

Figure 3.13 shows the magnetic field strength within a six ring-cusp NSTAR thruster. Highlighted is the 60 Gauss closed loop magnetic field contour line for that thruster and configuration. The closed loop contour line in this figure crosses the center axis of the thruster within the discharge chamber, indicating this magnet design will have a closed $360^{\circ}$ loop around the center axis.

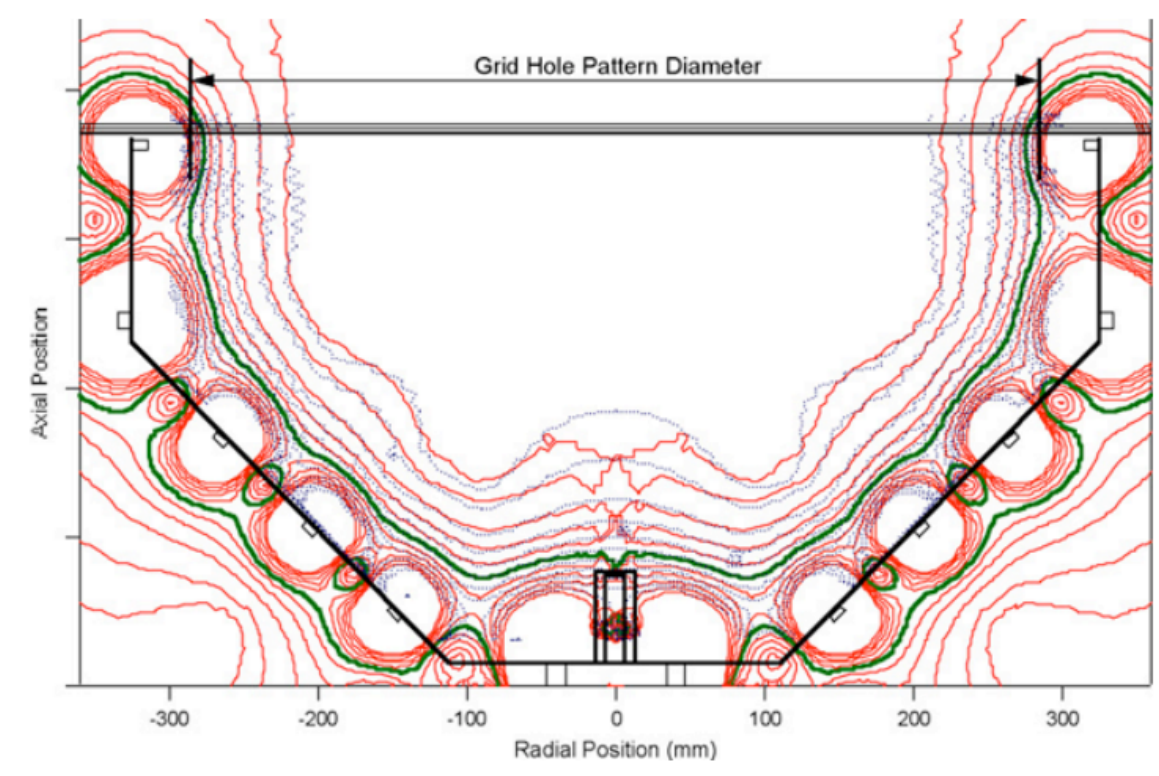

Figure 3.13: Magnetic Field Strength within 6 Ring Cusp NSTAR Thruster highlighting the 60 Gauss closed contour [15]

With the NSTAR thruster, the closed loop magnetic field strength is capable of reducing the ions lost to the anode wall by a factor of ten [12]. This significantly increases the 
efficiency of the plasma generator and reduces the discharge power required to produce the ion beam.

For the MiXI thruster, the closed loop transverse magnetic field strength is much greater in magnitude due to the smaller anode area and the reduced volume of the discharge chamber. In every configuration, there exists a saddle point in the magnetic field strength in a ring around the hollow cathode in the region between the base magnetic ring and the bottom anode ring cusp. The convergence of the closed loop occurs at this point but does not always yield a section that would create a $360^{\circ}$ loop, as is exemplified in Figure 3.13.

Similar to the analysis of the primary electron collision probability and the plasma potential, it was possible to alter the closed loop magnetic field strength within the computational model for the thruster. Figure 3.14 displays the trace of the fraction of the Bohm current lost to the anode wall as a function of the closed loop magnetic field strength.

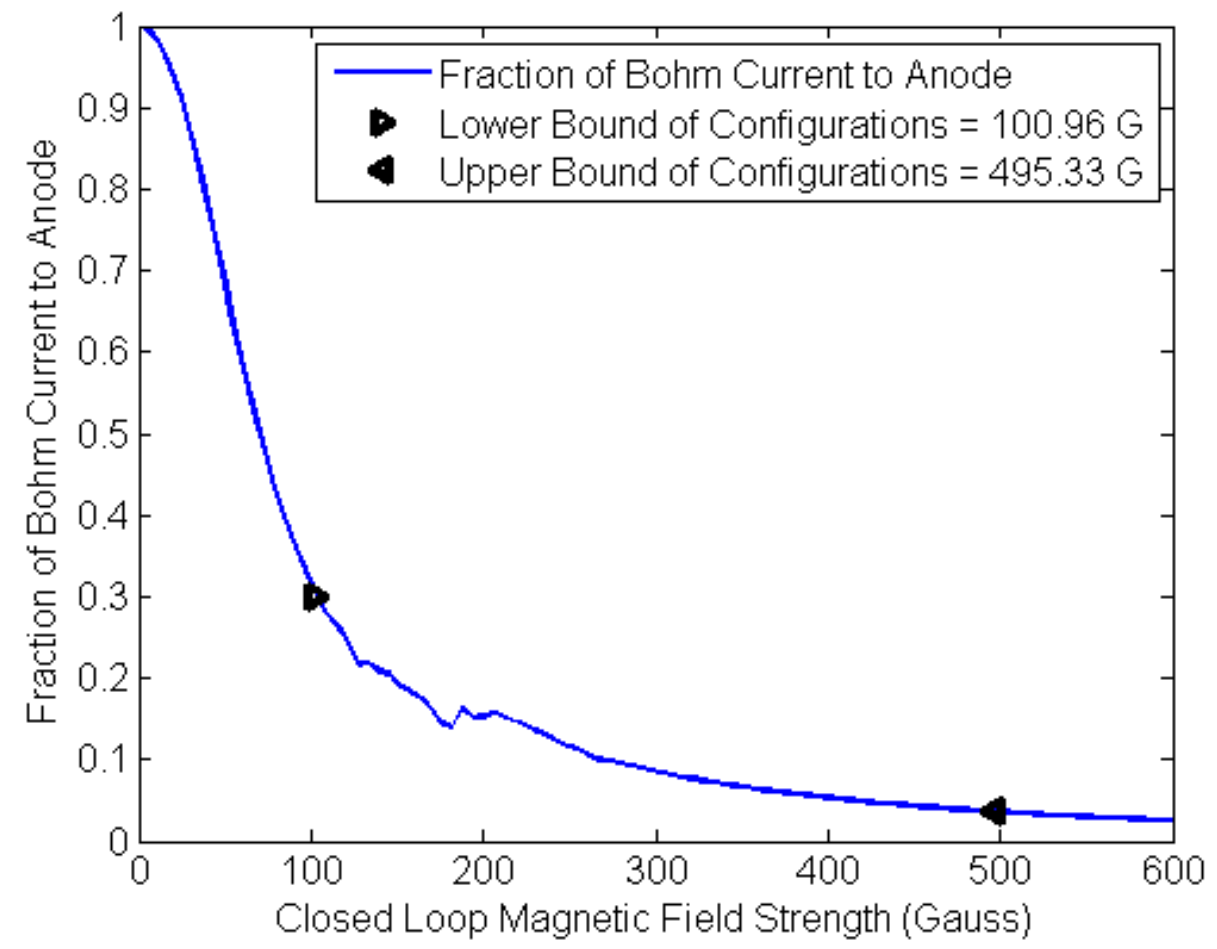

Figure 3.14: Effect on Confinement Factor by changing Closed Loop Magnetic Field Strength 
The markers on the confinement factor trace show the upper and lower bounds of the closed loop magnetic field strength for all the configurations analyzed in this thesis. The lower bound has a closed loop strength of $100.96 \mathrm{G}$ and corresponds to a confinement factor of 0.3002 , while the upper bound has a closed loop strength of $495.33 \mathrm{G}$ and corresponds to a confinement factor of 0.0359 . The wide variation in closed loop magnetic field strengths demonstrates that changes to the magnetic field design will have an effect on ion confinement within the discharge chamber and that the configurations of MiXI have reasonably good values of ion confinement.

\subsubsection{Other Considerations}

Aside from the numerical values of the closed loop and magnetic cusp field strengths, there were other aspects to the magnetic field design that were analyzed qualitatively. The main point of concern involves the centerline field strength and desires that the strength to be monotonically decreasing along the central axis past the cathode orifice. This idea was touched upon previously in Section 2.3 and drove the spacing of the discharge chamber and hollow cathode assemblies. A non-monotonically decreasing centerline field strength can cause the primary electrons emitted from the cathode to reflect at the orifice tip [12]. The plasma generation process is also altered and the 'bulb' of plasma that is usually present at the orifice tip can be more dispersed or pushed further downstream, reducing hollow cathode plasma generation performance.

Analysis of the baseline configuration shows a second peak after the primary peak at the orifice exit. This would indicate a reduction in efficiency of the hollow cathode and plasma generation processes, though this was not possible to quantitatively determine. Figures 3.15 and 3.16 show typical MATLAB outputs of the magnetic analysis of two different configurations. The nomenclature of the configurations and how they are achieved is discussed in Section 3.4. 

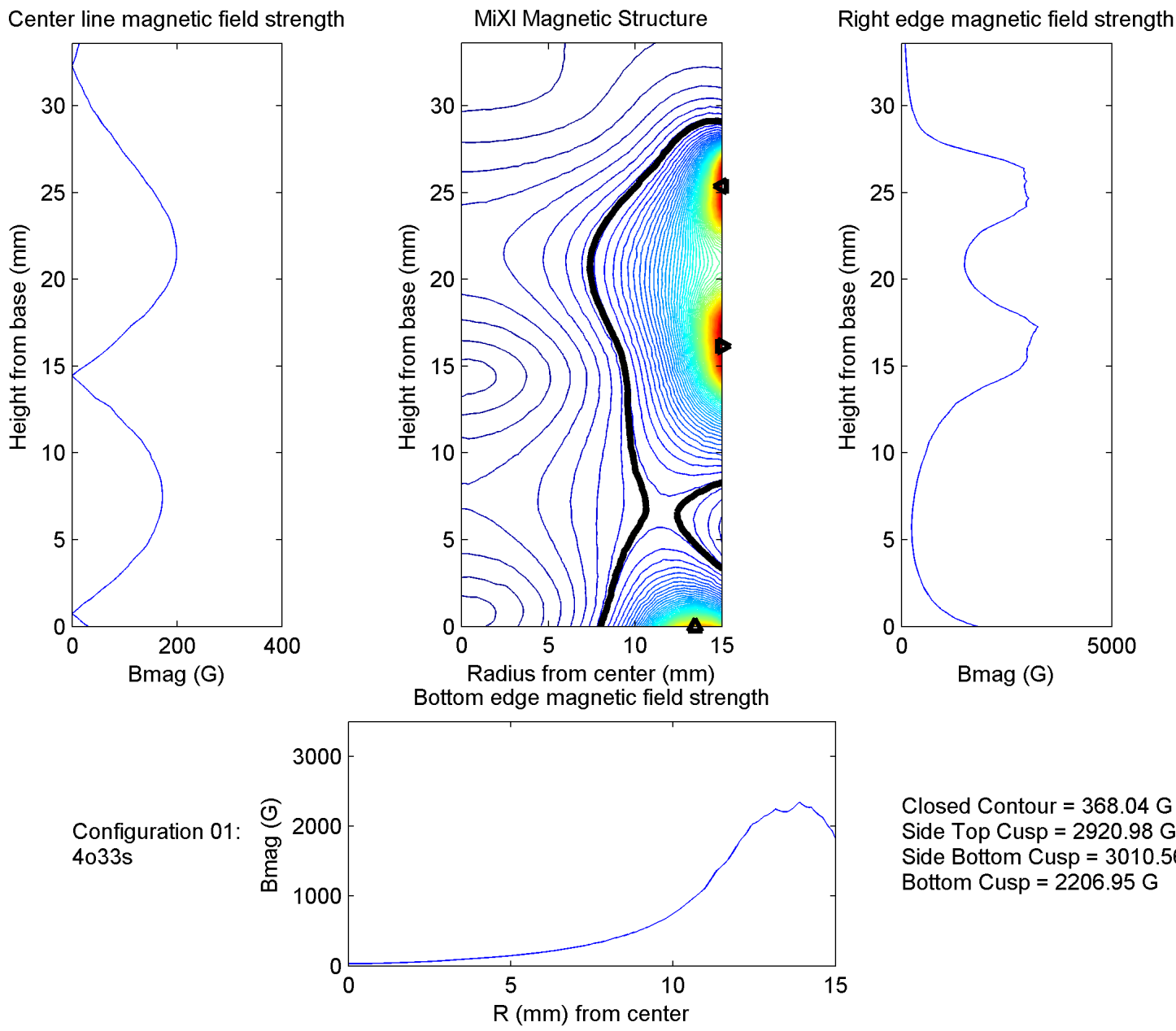

Closed Contour $=368.04 \mathrm{G}$

Side Top Cusp $=2920.98 \mathrm{G}$

Side Bottom Cusp $=3010.56 \mathrm{G}$

Bottom Cusp $=2206.95 \mathrm{G}$

Figure 3.15: Magnetic Field Analysis of Configuration 4o33s, baseline

Note the closed loop field strength contour shape and the secondary peak in the center line magnetic field trace. 


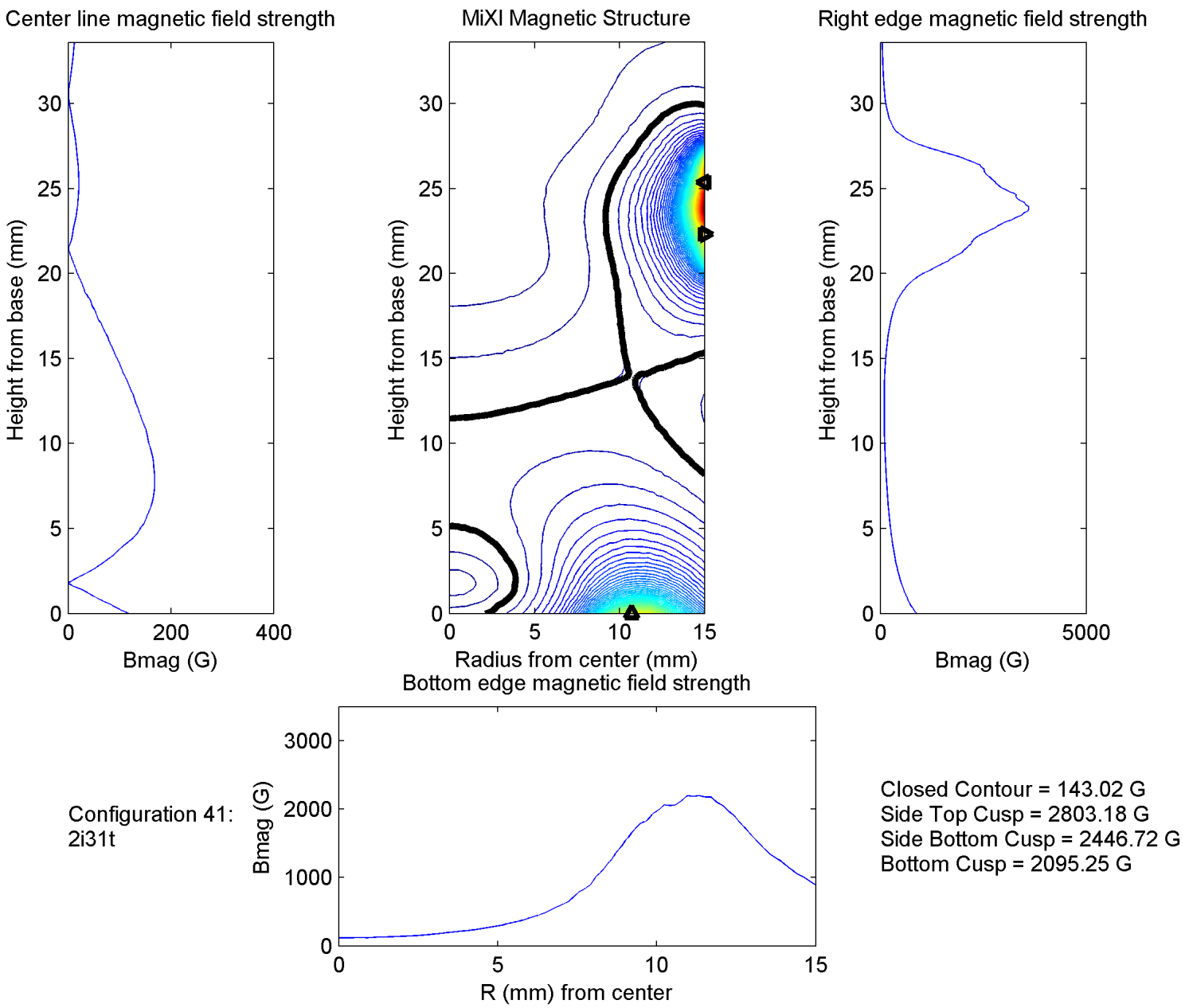

Figure 3.16: Magnetic Field Analysis of Configuration 2i31t

Note the $360^{\circ}$ closed loop field strength contour shape in this configuration and the lack of a second distinct peak in the center line magnetic field trace.

\subsection{Magnetic Model Variations}

One of the main goals of this thesis was to identify shortcomings to the current operation and make improvements to the performance of the thruster. Future work listed in previous theses $[19,22,38]$ identified the magnetic configuration and field analysis as an area of potential improvement. Creating the interconnected models and having the ability to alter 
the magnet configuration made it possible to see the effect changing the magnetic field has on the plasma parameters and overall performance.

The location and the strength of the magnets could be changed from the baseline configuration while still working within the physical confines of the anode pieces. There is a cavity between the Anode/Discharge Chamber piece and the Anode Magnet Mount with a $12.25 \mathrm{~mm}$ span. In the baseline configuration, the magnets are spaced to the top and bottom of that span; the location of the magnets and the extra space of the cavity can be seen in Figure 3.2 and in Figure 3.3. There is also a cavity created by the Base Magnet Mount which has a span of $5.85 \mathrm{~mm}$. The baseline configuration has the base magnet ring adjacent to the outer wall of the Base Magnet Mount; the location of the magnets and the cavity can be seen again in the aforementioned figures.

Different configurations of the magnets were considered that shifted their locations within the cavities allowed by the design of the parts. Changing the strength of the magnets was also considered and achieved by altering the number of magnets in the stacks. For ease, a simple labeling system was created to identify the different configurations; each configuration was specified by a five character code such as_4o33s.

- The leading character 4 represents the number of magnets in the Base Magnet Ring. The available configurations were $\mathbf{4}$ and $\mathbf{2}$.

- The following character $\mathbf{o}$ represents the location of the Base Magnet Ring. The available configurations were outside and inside, relative to the center axis.

- The third and fourth characters represented the number of magnets in the upper and lower Anode Magnetic Rings, respectively. The available configurations for each individual ring were $\mathbf{3}$ and $\mathbf{1}$ magnets.

- The last character $s$ represented the spacing of the Anode Magnetic rings. The available configurations were spread, bottom biased, or top biased. 
The label_4o33s represents the baseline configuration: four magnets in the bottom ring located outwards, three magnets in each anode ring spread to the top and bottom of the cavity. A total of forty-eight configurations were analyzed, considering all permutations of the magnet numbers and locations specified above.

Three new parts were designed that allowed the number and location of the anode and base magnetic rings to change to achieve these different configurations. The parts were designed in SolidWorks and consisted of a set of three types of rings: the Anode Cusp Spacer Ring, the Base Magnet Spacer Ring, and the Base Magnet Offset Ring. The geometry and materials are described later in Sections 3.4.1, 3.4.2, and 3.4.3, respectively.

With the design and materials of these new rings known, it was necessary to analyze the new configurations in FEMM and MATLAB as was done to the baseline configuration, described in Section 3.3. With the models in FEMM created in an easily editable fashion, it was possible to create new .dxf files of all the configurations simply. A sample of two new configuration meshes are shown in Figures 3.17a and 3.17b. 


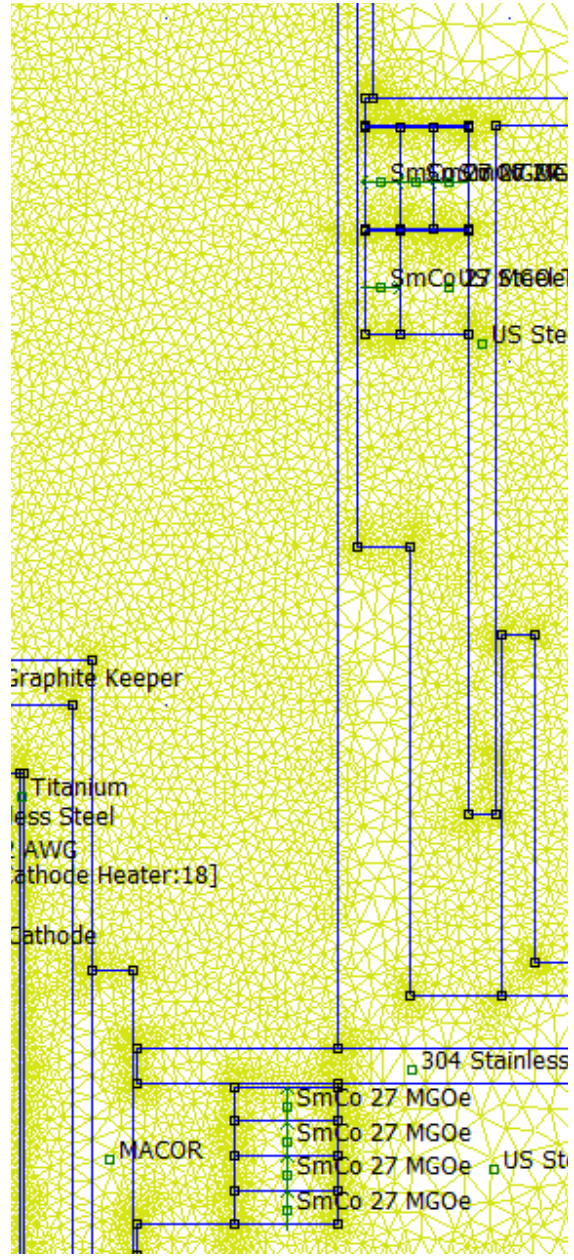

(a) Configuration_4o31t mesh

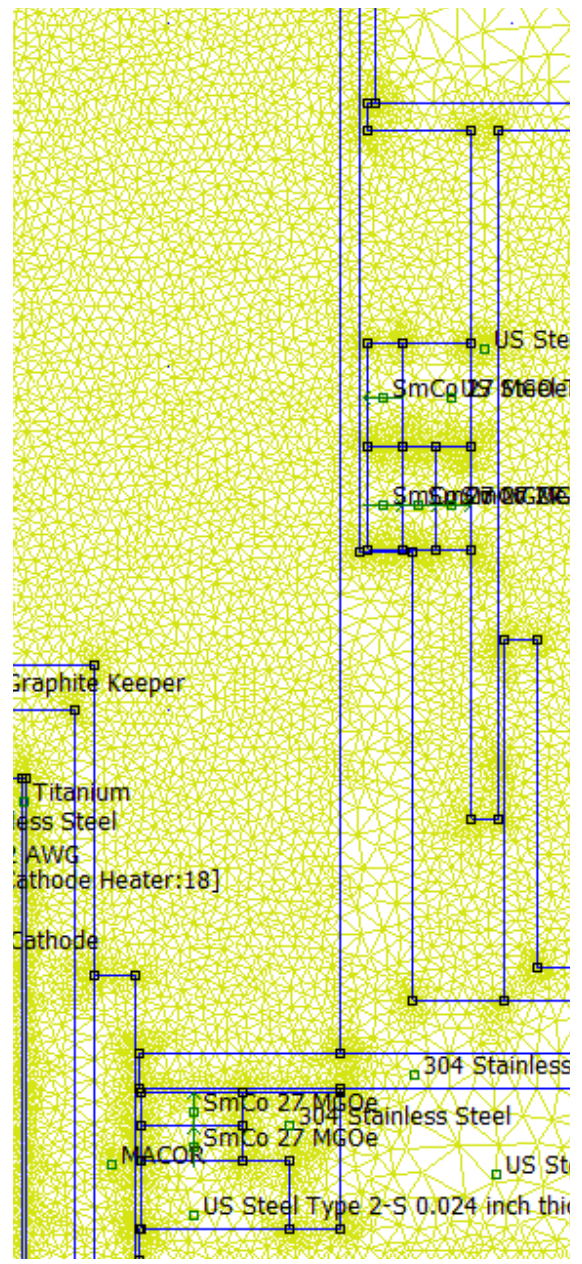

(b) Configuration_2i13b mesh

\section{Figure 3.17: Meshes of Two Sample Configurations}

In Figure 3.17a, it is possible to see the single magnet and spacer ring in the lower anode magnet ring and the top bias of both rings. In Figure 3.17b, it is possible to see the single magnet and spacer in the upper ring with the bottom bias of the anode magnet rings; also shown are the two base magnetic ring pieces and the position of the remaining magnets of the base magnet ring.

For every configuration, the magnetic field was solved for and output to MATLAB for analysis. The shape of the centerline strength and the geometry of the closed contour were 
noted for qualitative purposes. The cusp strengths and the closed loop contour strength were determined and used within the performance model calculations.

\subsubsection{Anode Cusp Spacer Ring}

The Anode Cusp Spacer Ring is a magnetic steel ring piece $2 \mathrm{~mm}$ wide, $3 \mathrm{~mm}$ tall and with an outer diameter of $37.5 \mathrm{~mm}$. This piece was built to replace two of the three magnets of the anode cusp ring while still providing an attachment point for the remaining magnet ring. Figures $3.18 \mathrm{a}$ and $3.18 \mathrm{~b}$ show the solid model representation of the anode cusp magnetic ring with the baseline three magnet stack compared to the magnetic ring with the spacer.

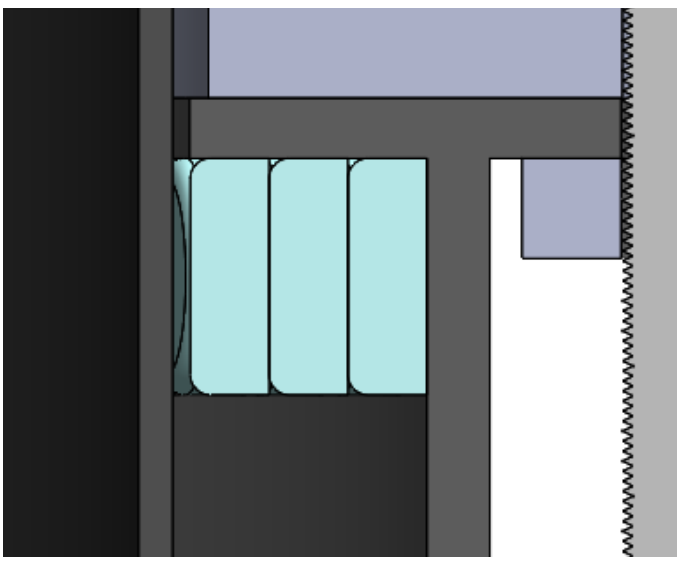

(a) Anode Cusp Magnetic Ring with the baseline three magnet stack

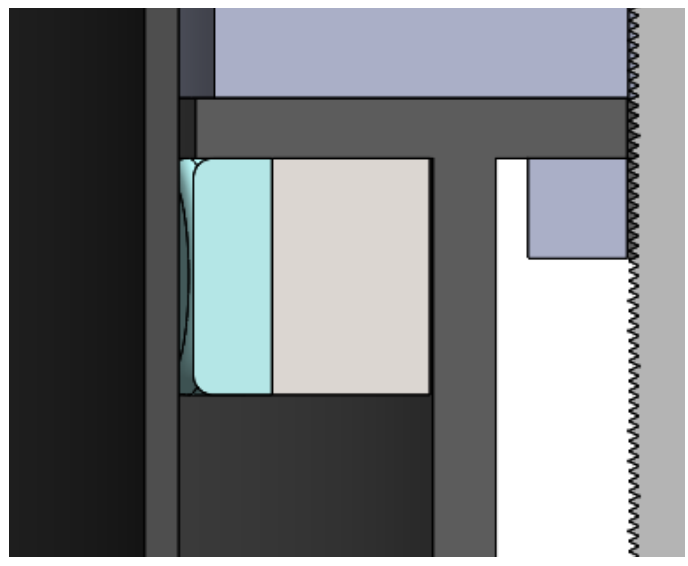

(b) Anode Cusp Magnetic Ring with the spacer installed

Figure 3.18: Anode Magnetic Ring Cusp Configuration Comparison between the baseline three magnet stack $3.18 \mathrm{a}$ and with the spacer installed $3.18 \mathrm{~b}$

One of these rings was built and installed in the lower anode magnetic ring of the thruster. The ring with the installed magnets, before being inserted into the anode, is shown in Figure 3.19. Due to complications with keeping the ring in place once it was installed, only a few different configurations were physically possible with this part. Further improvements to the design would undoubtedly yield a part capable of being used in some of the other configurations desired, as described in Section 3.4. 


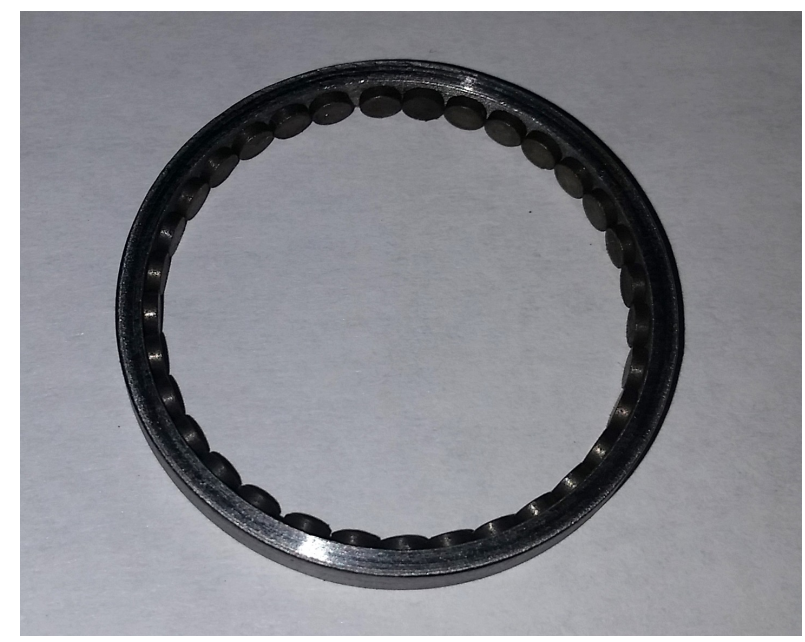

Figure 3.19: Anode Cusp Spacer Ring with installed magnets

\subsubsection{Base Magnet Spacer Ring}

The Base Magnet Spacer Ring was a magnetic steel ring designed to take the place of two of the magnets in the base magnetic ring. The ring is $2 \mathrm{~mm}$ tall, has an outer diameter of $27 \mathrm{~mm}$, and an inner diameter of $18.25 \mathrm{~mm}$. Figures 3.20a and 3.20b show the solid model representation of the base magnet spacer ring with the baseline four magnet stack compared to the magnetic ring with the spacer. 


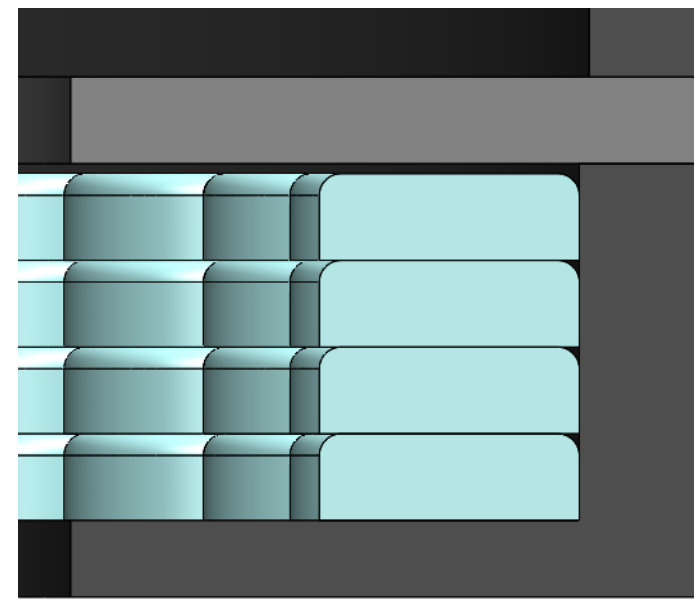

(a) Base Magnetic Ring with the baseline four magnet stack

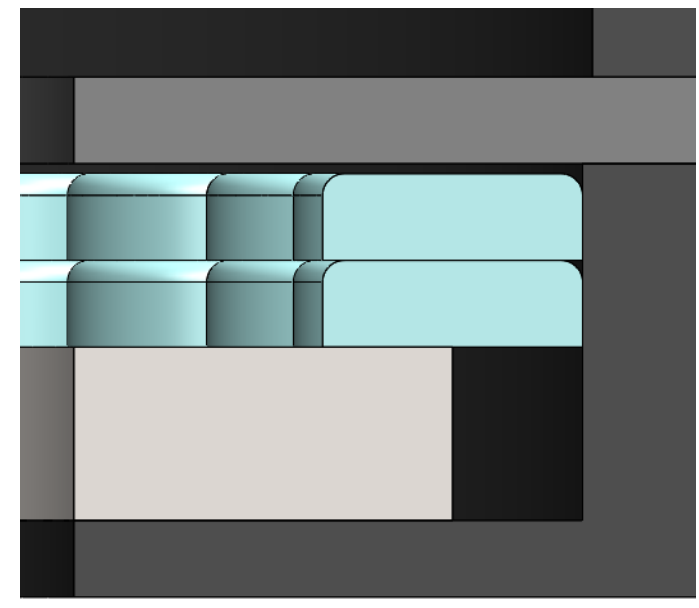

(b) Base Magnetic Ring with the spacer installed

Figure 3.20: Base Magnetic Ring Configuration Comparison between the baseline four magnet stack 3.20a and with the spacer ring installed 3.20b

This ring was manufactured and installed and used to change the base magnetic ring configuration to those specified in Section 3.4.

\subsubsection{Base Magnet Offset Ring}

The Base Magnet Offset Ring was an aluminum ring designed to offset the base magnetic ring towards the center axis of the thruster. The piece is a stepped ring with a height of $4 \mathrm{~mm}$, an outer diameter of $30 \mathrm{~mm}$, an inner diameter of $24.25 \mathrm{~mm}$, and a $2 \mathrm{~mm}$ tall step $1.5 \mathrm{~mm}$ wide. A solid model section view of the piece is shown in Figure 3.21. 


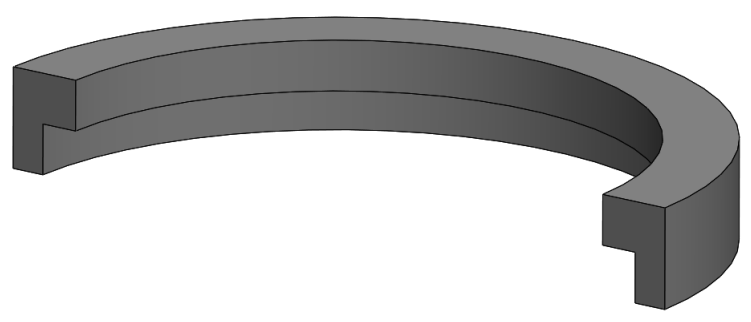

Figure 3.21: Base Magnet Offset Ring section view displaying the step cut, the actual design is a full ring

The step cut in the design allowed for this part to be used in conjunction with the Base Magnet Spacer Ring to change the base magnet ring configuration in two ways. The Base Magnet Offset Ring could be used independently to shift the whole stack of four magnets towards the center axis of the thruster, or it could be used to shift the remaining two magnets of the base magnet ring when the Base Magnet Spacer Ring is installed. The solid model representation of these two new configurations is shown in Figures 3.22a and 3.22b.
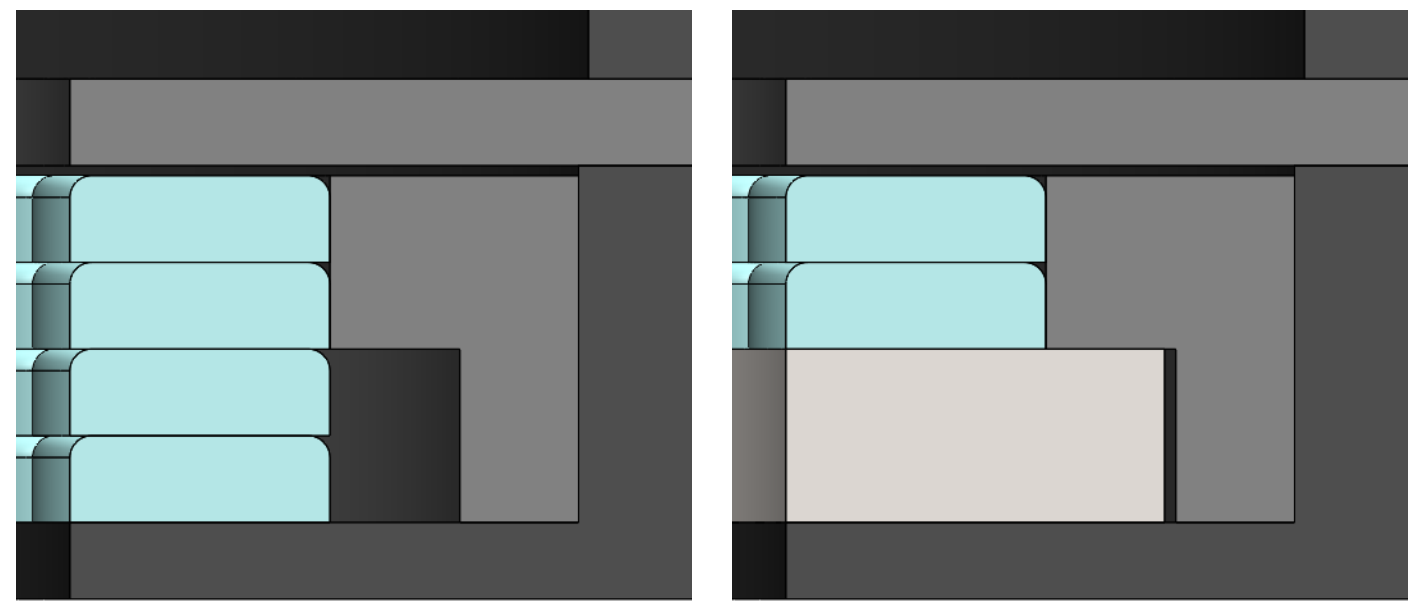

(a) Base Magnetic Ring with the baseline four

(b) Base Magnetic Ring with the spacer installed magnet stack

Figure 3.22: Base Magnetic Ring Configuration Comparison between the baseline four magnet stack offset by the ring 3.22a and with the spacer ring also installed to only offset the remaining magnet pair $3.22 \mathrm{~b}$ 
Due to time constraints and availability, this component was not manufactured. The configurations specified in Section 3.4 were analyzed through the course of this thesis, but the actual thruster could not be tested with the configurations involving this component. 
Chapter 4

\title{
COMPUTATIONAL MODEL
}

\begin{abstract}
A computational model was developed in order to approach the design of an ion thruster analytically. This model utilizes processes developed primarily by Wirz and Goebel to internally calculate the plasma parameters and processes of the thruster to find a closed form model of the ion thruster [12]. All parts of the code were written by the author and based on previously constructed and verified models [15]. The model consists of a number of discrete parts and was organized in a fashion shown in Figure 4.1.
\end{abstract}




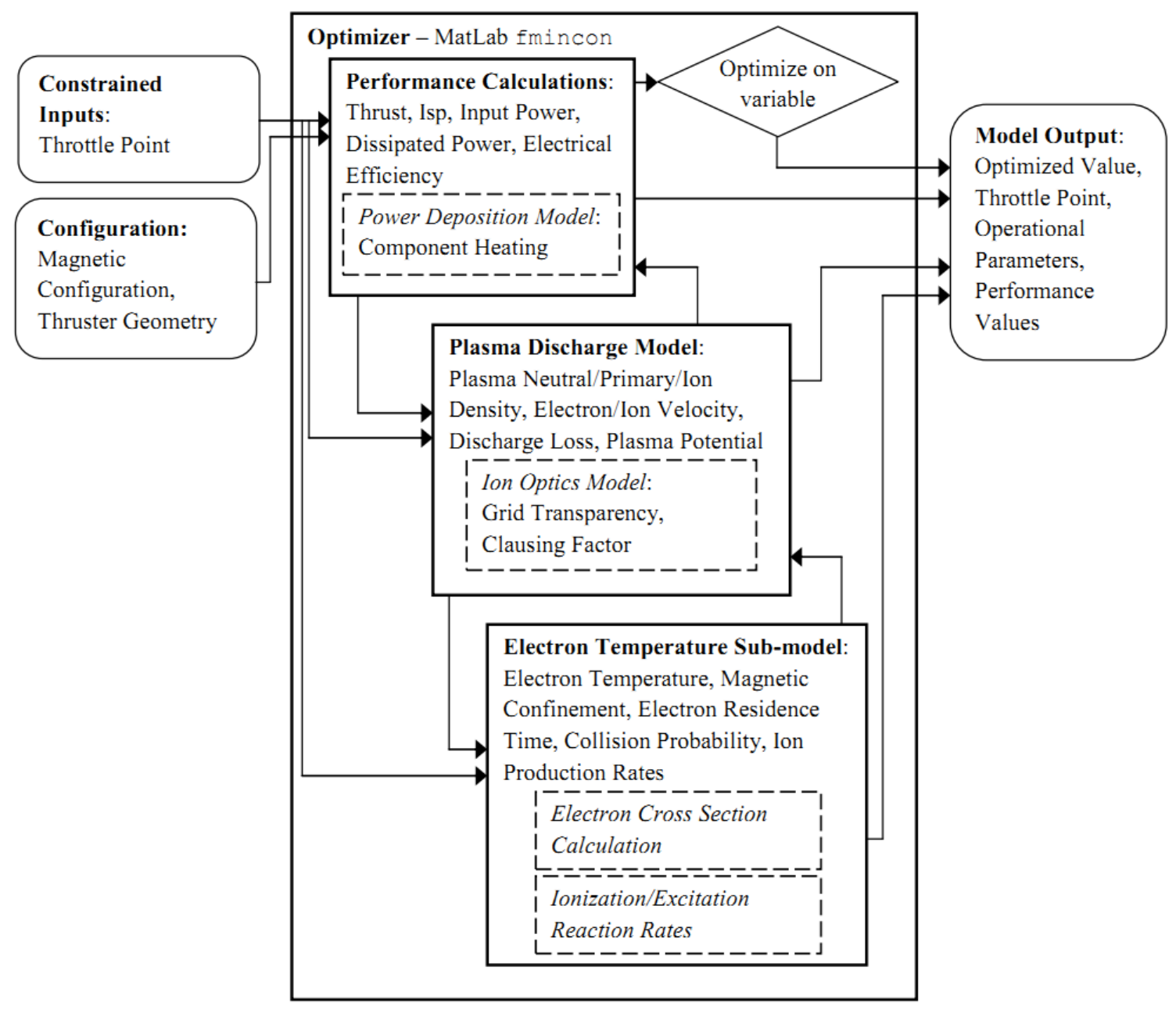

Figure 4.1: Functional flow chart of Optimization code

\subsection{Optimization Code Overview}

The optimization in this thesis was performed by the MATLAB function fmincon, a nonlinear constrained multi-variable function minimization routine. Detailed information about fmincon and its use can be found in the MATLAB source documentation [23].

The function operates on a user defined objective function with any number of inputs and a single output, called the objective. For this model, the objective function was written to allow for five constrained inputs and had the choice to minimize and output one of six 
objective variables solved within the code. When solving, MATLAB changes the value of the inputs with respect to user defined bounds to determine the minimum of the objective, thus finding the optimum solution to the function.

While the optimizer can operate on a single variable objective, a more involved cost function can be utilized to determine the optimum throttle point based on any number of objective variables. The cost function can be created consisting of weighted relations between two or more outputs, such as total efficiency and dissipated power. While different optimization tools can operate and determine solutions based on constrained inputs and constrained outputs on multiple objectives, fmincon can only operate with constrained inputs on a single objective; developing and utilizing a cost function can expand the power of fmincon to determine throttle points that satisfy the conditions of the cost function.

fmincon can be configured within MATLAB to based on the objective function and the variable inputs. Changing the solution algorithm, the tolerances, or the finite difference approximation solver can effect the run time and the solution from fmincon. The source documentation with MATLAB [26] and online support at MathWorks [25] contains all the information on configuring fmincon. The configuration algorithm used in this thesis was 'sqp', Sequential Quadratic Programming, and used central finite differencing techniques in the solver. The optimizer was set with a termination tolerance on the function value of

$10^{-6}$. These options were able to iterate and solve the model in this thesis accurately and in a timely fashion acceptable to the author.

\subsubsection{Constrained Inputs}

There were a total of six variables input to the objective function and fmincon. These values included: 
- Beam Current $I_{B}$

- Mass Utilization Efficiency $\eta_{m}$

- Discharge Voltage $V_{d}$

- Screen Grid Voltage $V_{s g}$

- Acceleration Grid Voltage $V_{a g}$

These values were the inputs to the objective function with upper and lower bounds set by the user. The limits placed on these inputs were based on realistic values of what they represent or what the lab equipment was capable of. Certain variables would reach their constraints during the optimization routine, meaning that further improvements may be possible if the constraints were altered or removed. When setting constraints, values could be specified and held constant by setting the upper and lower bounds to the input values. For some test cases, it was beneficial to set the beam current to a specific value and optimize around the other variables.

The constrained inputs represented throttle settings for operation and the thruster configuration. In the cases discussed and presented in this thesis, the geometry of the thruster was not altered because the results of the optimization was tested against the actual thruster. However, it would be possible to run optimization routines varying all aspects of the thruster geometry as well. The code and models were written with the mindset that it could be expanded and used on any size and configuration of thruster.

It would have been possible to allow the optimizer to control the magnetic parameters and the geometry of the thruster in the process of determining the optimum solutions. However, the optimizer is provided the configuration of the thruster to allow for testing against the optimizer outputs with the MiXI-CP-V3 thruster. While the optimizer would be capable of finding the optimal magnetic field strengths and geometry as well as the throttle values, it is not necessarily going to be possible to recreate that structure or build that magnetic field to test against. 


\subsection{Performance Model}

The model self-consistently calculates many internal parameters used within and passed between the following sub-models, including the electron temperature, the neutral gas, primary electron, and plasma densities, the plasma potential, the discharge current, and the ion currents to components of the discharge chamber. The models also assume a near uniform plasma, a quasi-neutral charge distribution, a mono-energetic primary electron energy, and a constant temperature within the plasma [12]. These assumptions are inconsistent with reality, but the following performance model formulation has been shown to accurately predict the performance of the NEXT and NEXIS ion thrusters [15] and it was determined to be suitable for use modeling MiXI.

There are known limitations to using this model for small ion thrusters and small discharge chambers. As the discharge chamber size decreases, the ratio of the surface area to volume drastically changes and significantly impacts the performance of the thruster. The OD model requires considering the entire discharge chamber as a homogeneous plasma when performing calculations. Testing on similar thrusters [22] demonstrate that there is a spatial variance to the plasma and processes of the ion thruster that cannot be fully realized using a OD model.

\subsubsection{Electron Temperature Sub-Model}

The calculation of Electron Temperature $T_{e V}$ was a highly critical aspect of this code. The process for determining the electron temperature is well defined in the "Analytical Ion Thruster Discharge Performance Model" by Goebel and Wirz [15]. The solution to find the electron temperature requires another minimization of variables; the equations and processes for the solution are described below.

The solution to electron temperature requires a number of inputs listed here: 
- Electron Temperature Approximation $T_{e V}$

- Beam Current $I_{B}$

- Mass Utilization Efficiency $\eta_{m}$

- Discharge Current $I_{d}$

- Discharge Voltage $V_{d}$

- Plasma Potential $\phi$

Many of these inputs are controlled by the optimization loop, requiring the calculation of electron temperature to be re-run every iteration of the greater loops.

The electron temperature in the discharge chamber is calculated from a particle balance of the ions. The ion production rate must equal the ion loss rate, values that are both determined by the electron temperature. An iterative process varying the approximation of $T_{e V}$ will converge and the true electron temperature can be determined. The particle balance equation is

$$
\frac{\sqrt{\frac{k T_{e V}}{M}}}{\left\langle\sigma_{I} v_{e}\right\rangle+\frac{n_{p}}{n_{e}}\left\langle\sigma_{I} v_{p}\right\rangle}=\frac{2 n_{0} V}{A_{i}}
$$

and the equations and steps required are as follows.

Knowing the beam current and the screen transparency, the ion screen current can be easily determined

$$
I_{s g}=I_{b} \frac{1-T_{s}}{T_{s}}
$$

The average neutral gas velocity $v_{0}[\mathrm{~m} / \mathrm{s}]$ out of the chamber is calculated by

$$
v_{0}=\sqrt{\frac{8 T_{0} k}{\pi M}}
$$

The propellant flow rate $Q_{i n}$ [Standard Cubic Centimeters per Minute (SCCM)] is calculated by converting the beam current from its equivalent amperes [12]

$$
Q_{i n}=I_{b} / 7.174486 \times 10^{-2}
$$


The ion acoustic velocity $v_{a}[\mathrm{~m} / \mathrm{s}]$ is calculated using the input approximation of electron temperature by

$$
v_{a}=\sqrt{\frac{T_{e V} e}{M}}
$$

The ion acoustic velocity is also known as the Bohm Velocity $v_{B o h m}$ in literature and and referred to as such in parts of this report.

With these values and other inputs, the neutral gas density $n_{0}$ [particles $/ m^{3}$ ] can be calculated by

$$
n_{0}=\frac{4 Q_{i n} 4.477962 \times 10^{17}\left(1-\eta_{m}\right)}{\eta_{m} v_{0} A_{s g} T_{p} \eta_{c}}
$$

The average plasma density $n_{e}$ [particles $/ m^{3}$ ] is calculated by

$$
n_{e}=\frac{2 I_{b}}{T_{s} v_{a} A_{s g} e}
$$

In order to determine the confinement factor and the ion loss area, an understanding of the magnetic field structure is required. The magnetic fields within the discharge chamber are discussed in the Magnetic Field Analysis Section (3.3). For this simplified model considering bulk averages and the aforementioned assumptions, it is only required to know the closed loop magnetic field strength and the strength at the surface of the discharge chamber at the magnetic cusps. A more in depth code would consider a spatially resolved discharge chamber and the $2 \mathrm{D}$ effects of the changing magnetic field on the associated plasma parameters.

The electron-ion collision frequency $v_{e i}$ [collisions/sec] is calculated by

$$
v_{e i}=\frac{2.9 \times 10^{-12} n_{e} \ln \Lambda}{T_{e V}^{1.5}}
$$

where $\ln \Lambda$ is the Coulomb Logarithm defined by

$$
\ln \Lambda=23-0.5 \ln \left(\frac{10^{-6} n_{e}}{T_{e V}^{3}}\right)
$$

The electron-neutral collision frequency $v_{e 0}$ [collisions/sec] is defined by

$$
v_{e 0}=\sigma_{i} n_{0} \sqrt{\frac{8 e T_{e V}}{\pi m_{e}}}
$$


where the electron collision cross section $\sigma_{i}\left[\mathrm{~m}^{2}\right]$ is based on electron temperature and is defined by

$$
\sigma_{i}=6.6 \times 10^{-19}\left(\frac{\frac{T_{e V}}{4}-0.1}{1+\left(\frac{T_{e V}}{4}\right)^{1.6}}\right)
$$

The total electron collision frequency $v_{e}$ [collisions/sec] is the sum of the constituent collision frequencies

$$
v_{e}=v_{e 0}+v_{e i}
$$

and the electron mobility $\mu_{e}$ is defined as

$$
\mu_{e}=\frac{e v_{e}}{m_{e}}
$$

The total ion loss area $A_{t}\left[\mathrm{~m}^{2}\right]$ is determined by the sum of the screen grid area $A_{s g}$ and the anode $A_{s a}$ reduced by the confinement factor

$$
A_{i}=A_{s g}+A_{s a} f_{c}
$$

The ion loss rate is a combination of the previous terms and is written as

$$
\text { ionloss }=\frac{2 n_{0} V}{A_{i}}
$$

The peak potential in the plasma $V_{p p}[\mathrm{eV}]$ is approximated from the plasma potential and the electron temperature

$$
V_{p p}=\phi+T_{e V} / 2
$$

and the primary energy $V_{p e}[\mathrm{eV}]$ of the electrons out of the cathode is

$$
V_{p e}=V_{d}+V_{p p}+5 / 2 T_{h}
$$

and knowing the potential distribution within the plasma, the keeper potential $V_{k}$ can be estimated simply by

$$
V_{k}=V_{p e}-V_{c}
$$


The value $V_{c}$ represents the cathode voltage drop and is either measured inside the hollow cathode or calculated using higher order hollow cathode models. The value is typically on the order of 5-10 $\mathrm{V}$ for larger thrusters with similar discharge voltages as what would be expected from MiXI [12]; a constant value of $5 \mathrm{~V}$ was used in these calculations and should provide a reasonable estimation for this model. The component $5 / 2 T_{h}$ represents the cathode convective energy [12]. The primary energy is used to calculate the primary electron velocity out of the cathode by

$$
v_{p}=\sqrt{2 \frac{e}{m_{e}} V_{p e}}
$$

The keeper current $I_{k}$ is usually a very small value representing the summation of the ion and electron collection rates to the exposed keeper. Previous work has indicated a relationship between the average beam current density and the keeper current density described by the equation

$$
I_{k}=\left(\frac{1.167 I_{b}}{A_{s g}}+0.283\right) A_{k}
$$

The keeper area $A_{k}$ is the total area of the keeper exposed to the discharge plasma. This empirical relationship was determined from tests on the NSTAR and NEXT ion thrusters [9]. However, through testing, the MiXI keeper often showed current values much larger than would be predicted through this relationship.

The primary electron loss area at the anode cusps $A_{p}\left[m^{2}\right]$ is calculated as

$$
A_{p}=\sum_{i=1}^{n} \frac{2 L_{c u s p, i}}{B_{c u s p, i}} \sqrt{2\left(V_{p e}-V_{p p}\right) m_{e} / e}
$$

where $n$ is the number of magnetic cusps present in the ion thruster. For MiXI there are two cusps at the anode wall and one at the anode base, however the anode base ring is often not considered for the primary loss area [38]. For MiXI, the length of the wall cusps are identical and, for some configurations, the magnetic field strength is also identical, but the code is written to handle any number of cusps for any type of thruster. 
The hybrid loss area $A_{h}\left[\mathrm{~m}^{2}\right]$ represents the area where both ions and electrons can collide with the thruster walls and dissipate, given by

$$
A_{h}=\sum_{i=1}^{n} \frac{4 L_{\text {cusp }, i}}{B_{\text {cusp }, i}} \sqrt{\sqrt{\frac{2 T_{e V} m_{e}}{e}} \sqrt{\frac{2 T_{i} M}{e}}}
$$

where $n$ is again the number of cusps to consider.

The atomic cross section and the reaction rate coefficients for the excited and ionized Xenon atoms are dependent on electron temperature. Approximations based on experimental data have been developed and are presented below.

Cross Section Approximation The cross section of Xenon is generally approximated using the electron temperature with fits similar to Figure 4.2.

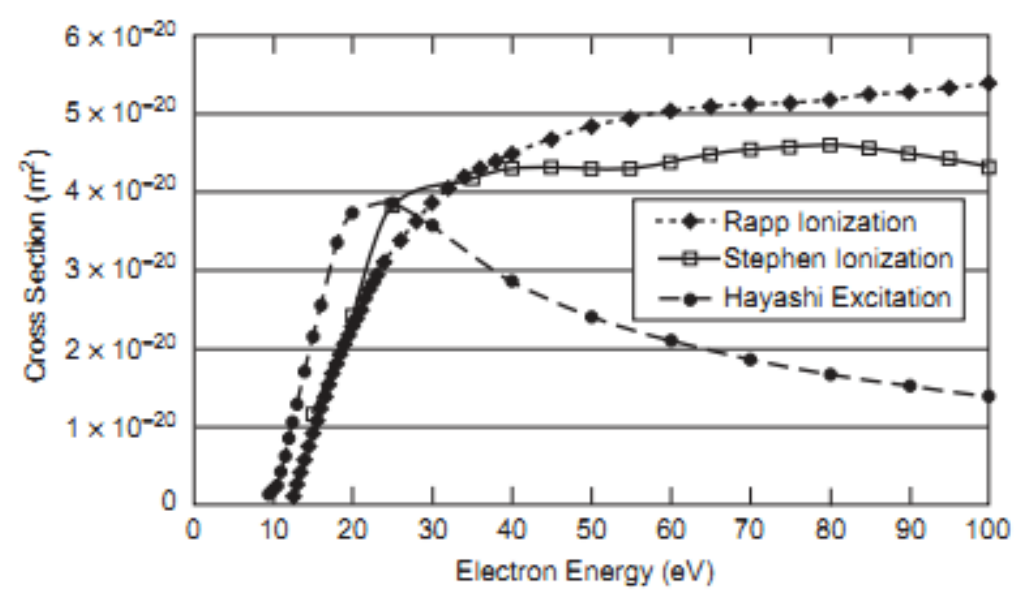

Figure 4.2: Ionization and Excitation Cross Sections for Xenon [12]

This range of data is not very applicable to micro ion thrusters as they tend to operate in a region of lower electron energy [38]. The following equations were developed based off of curve fits and approximations for lower energy plasma that utilize the primary electron 
energy.

$$
\begin{aligned}
\sigma_{I}= & 8.79 \times 10^{-21}\left(-8.33+0.955 V_{p e}-0.0273 V_{p e}^{2}+0.000401 V_{p e}^{3}\right. \\
& \left.-2.94 \times 10^{-6} V_{p e}^{4}+8.55 \times 10^{-9} V_{p e}^{5}\right) \\
\sigma_{E}= & 2.6745 \times 10^{-19}-8.8347 \times 10^{-20} V_{p e}+1.1036 \times 10^{-20} V_{p e}^{2}-6.8313 \times 10^{-22} V_{p e}^{3} \\
& +2.44 \times 10^{-23} V_{p e}^{4}-5.3694 \times 10^{-25} V_{p e}^{5}+7.4029 \times 10^{-27} V_{p e}^{6}-6.2331 \times 10^{-29} V_{p e}^{7} \\
& +2.9302 \times 10^{-31} V_{p e}^{8}-5.8928 \times 10^{-34} V_{p e}^{9} \\
\sigma_{D}= & -1.0846 \times 10^{-19}+9.9962 \times 10^{-21} V_{p e}-2.8328 \times 10^{-22} V_{p e}^{2} \\
& +3.8017 \times 10^{-24} V_{p e}^{3}-2.4497 \times 10^{-26} V_{p e}^{4}+6.0962 \times 10^{-29} V_{p e}^{5}
\end{aligned}
$$

The subscripts $I, E$, and $D$ represent Ionization, Excitation, and Double ion respectively. The cross sections are in units of $\left[\mathrm{m}^{2}\right]$.

Ionization and Excitation Reaction Rates Similar to the cross section of Xenon, the Reaction Rates are dependent upon electron temperature [12]. The reaction rates are the cross sections multiplied by the averaged Maxwellian electron velocity and represent the rate of production of charged atoms in the plasma. Ionization, Excitation, and Double Ion reaction rates are determined by curve fits to experimental data and are presented below.

$$
\begin{aligned}
\left(T_{e V}<5\right)\left\langle\sigma_{I} v_{e}\right\rangle= & 10^{-20}\left(\left(3.97+0.643 T_{e V}-0.0368 T_{e V}^{2}\right) \exp \frac{-12.127}{T_{e V}}\right) \sqrt{\frac{8 e T_{e V}}{\pi m_{e}}} \\
\left(T_{e V}>5\right)\left\langle\sigma_{I} v_{e}\right\rangle= & 10^{-20}\left(-1.031 \times 10^{-4} T_{e V}^{2}+6.386 \exp ^{\frac{-12.127}{T_{e V}}}\right) \sqrt{\frac{8 e T_{e V}}{\pi m_{e}}} \\
\left\langle\sigma_{E} v_{e}\right\rangle= & 1.93 \times 10^{-19} \frac{\exp ^{\frac{-11.6}{T_{e V}}}}{\sqrt{T_{e V}}} \sqrt{\frac{8 e T_{e V}}{\pi m_{e}}} \\
\left\langle\sigma_{D} v_{e}\right\rangle= & -2.8383 \times 10^{-16}+6.0557 \times 10^{-16} T_{e V}-3.8833 \times 10^{-16} T_{e V}^{2} \\
& +7.4512 \times 10^{-17} T_{e V}^{3}+3.6717 \times 10^{-18} T_{e V}^{4}-1.0976 \times 10^{-18} T_{e V}^{5} \\
& +4.7475 \times 10^{-20} T_{e V}^{6}
\end{aligned}
$$


The approximation for the double ion reaction rate is not as well known as the other approximations and is only valid for the span of electron temperature between 2.3 and $10 \mathrm{eV}$. The reaction rate coefficients are in units of $\left[\mathrm{m}^{3} / \mathrm{sec}\right]$.

The primary ionization and excitation reaction rates can then be calculated from the cross section approximations

$$
\begin{aligned}
\left\langle\sigma_{I} v_{p}\right\rangle & =\sigma_{I} \sqrt{\frac{2 e V_{p e}}{m_{e}}} \\
\left\langle\sigma_{E} v_{p}\right\rangle & =\sigma_{E} \sqrt{\frac{2 e V_{p e}}{m_{e}}}
\end{aligned}
$$

and the total primary collision cross section from the sum of the ionization and excitation cross sections

$$
\sigma_{p}=\sigma_{I}+\sigma_{E}
$$

The probability of collision of a primary electron with a neutral molecule is calculated as

$$
P=1-\exp \left(\frac{-n_{0} \sigma_{p} V}{A_{p}}\right)
$$

The collision probability can be used as a metric of how effective the primary electron discharge is at interacting with the neutral gas element.

The last piece of information needed to calculate electron temperature is the primary electron plasma density $n_{p}$. This value is calculated through determining the total confinement time of the electrons in the discharge chamber. The total confinement time $t_{t}$ [sec] is the inverse of the sum of the inverse of the three residence times

$$
t_{t}=\frac{1}{\left(\frac{1}{t_{s}}+\frac{1}{t_{c}}+\frac{1}{t_{m}}\right)}
$$

The time to allow for high energy primary electrons to slow a Maxwellian distribution was determined by Sptizer [35] and is approximated using his G-function.

$$
t_{s}=\frac{\omega}{2 A_{d} l_{f 2} G}
$$


The plasma frequency $\omega[$ cycles/sec] is calculated by

$$
\omega=100 \sqrt{\frac{2 e V_{p e}}{m_{e}}}
$$

A diffusion constant $A_{d}$ is calculated as

$$
A_{d}=\frac{8 \pi e_{2}^{4} n_{e} 10^{-6}\left(23-0.5 \ln \left(\frac{10^{-6} n_{e}}{T_{e V}^{3}}\right)\right)}{\left(1000 m_{e}\right)^{2}}
$$

The inverse mean velocity $l_{f 2}$ is calculated in $c g s$ units

$$
l_{w}=\frac{1000 m_{e}}{2 \times 10^{7} T_{e V} e}
$$

The G-function is

$$
G=0.4638\left(100 l_{w}\right)^{-1.957}
$$

with $l_{w}$ as

$$
l_{w}=\sqrt{l_{f 2} \frac{2 e V_{p e}}{m_{e}}}
$$

The primary thermalization time for electrons due to collisions is

$$
t_{c}=\frac{1}{n_{0} \sigma_{p} \sqrt{\frac{2 e V_{p e}}{m_{e}}}}
$$

The primary ballistic confinement time $t_{m}$ within the volume is

$$
t_{m}=\frac{V m_{e}}{2 e A_{p} \sqrt{V_{p e}-V_{p p}}}
$$

The primary electron plasma density $n_{p}$ [particles $/ \mathrm{m}^{3}$ ] can then be determined from the total confinement time by

$$
n_{p}=\frac{\left(I_{d}-I_{s g}\right) t_{t}}{V e}
$$

and the ion production rate of the ion particle balance equation can be solved as

$$
\text { ionproduction }=\frac{\sqrt{\frac{k T_{e V}}{M}}}{\left\langle\sigma_{I} v_{e}\right\rangle+\frac{n_{p}}{n_{e}}\left\langle\sigma_{I} v_{p}\right\rangle}
$$


With both halves of the ion balance known, equation 4.1 is iteratively solved for the electron temperature. As this is a single variable problem, the MATLAB function fminbnd was used. The function fminbnd is a bounded minimization solver [24] and was able to find the value of $T_{e V}$ that balanced the ion production/loss equation with a solution tolerance of $10^{-6}$. The values for electron temperature were generally between 2-4 eV and are consistent with other thrusters of this class $[38,12]$.

\subsubsection{Plasma Discharge Sub-Model}

In order to solve for the internal plasma parameters and properties of the discharge within the anode, it is critical to determine the discharge current of the system. The discharge current and other plasma parameters are calculated following the work of Goebel and Wirz $[15,12]$. The solution for discharge current requires a number of inputs listed here:

- Discharge Current (Initial Approximation) $I_{d}$

- Beam Current (Desired) $I_{B}$

- Mass Utilization Efficiency (Desired) $\eta_{m}$

- Discharge Voltage (Desired) $V_{d}$

- Plasma Potential (Approximation) $\phi$

- Confinement Factor (Approximation) $f_{c}$

The plasma discharge sub-model will use an approximated input of the discharge current, the plasma potential, and the confinement factor to determine those values which will meet the desired beam current, mass utilization, and discharge voltage.

Using the approximation of the discharge current, the inputs listed above, and the additional initial guess for electron temperature, the balanced electron temperature of the plasma can be solved using the method in Section 4.2.1. With $T_{e V}$ known, the true discharge current can be determined by finding the discharge current that solves the current 
balance within the anode. A representation of the current sources and sinks within the discharge chamber is shown in Figure 4.3 below.

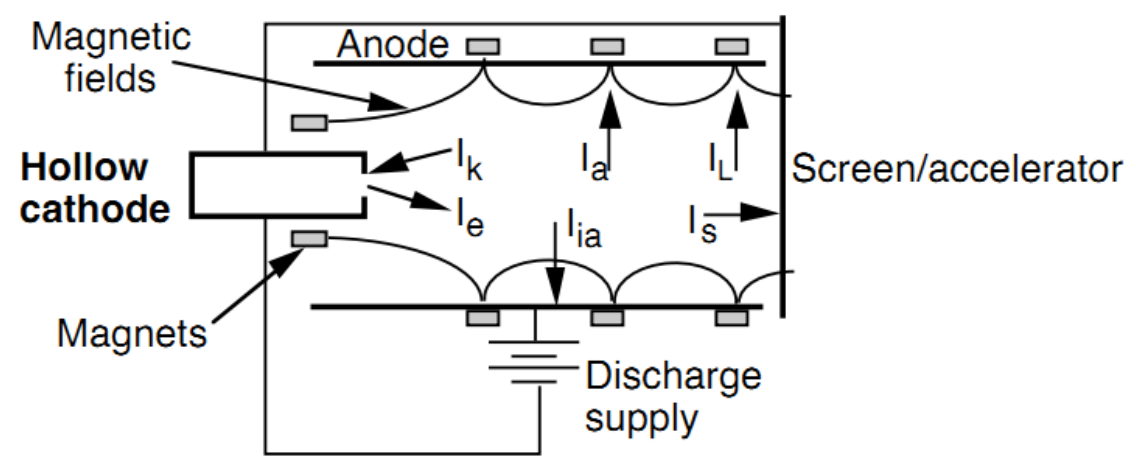

\section{Figure 4.3: Current Distribution within the Discharge Chamber [15]}

With some values of the plasma solved for in the iteration of electron temperature already known, the discharge loss can be calculated. The discharge loss $\eta_{d}$ [eV/ion] is a measure of efficiency representing the power required to produce ions within the discharge plasma, and is a good metric of discharge chamber performance [12].

To determine the discharge current, it is necessary to understand the power flow within the discharge chamber. The input power is

$$
P_{i n}=I_{e}\left(V_{d}-V_{c}+\phi\right)
$$

and the power out of the discharge is

$$
P_{\text {out }}=I_{p} U^{+}+I^{*} U^{*}+\left(I_{s g}+I_{k}\right)\left(V_{d}+\phi\right)+\phi\left(I_{b}+I_{i a}\right)+I_{a}\left(2 T_{e V}+\phi\right)+I_{L}\left(V_{d}-V_{c}+\phi\right)
$$

$I_{p}$ is the total number of ions produced in the discharge, $U^{+}$is the ionization potential of Xenon, $I^{*}$ is the total number of excited atoms produced in the discharge, and $U^{*}$ is the excitation potential of Xenon. $I_{s g}$ is the number of ions through the screen plane, $I_{k}$ is the ion back-flow current to the cathode, $I_{a}$ is the electron current to the anode, $I_{i a}$ is the ion current lost to the anode, and $I_{L}$ is the primary electron current fraction lost to the anode. 
With the screen grid commonly connected to the cathode potential, conservation of particles yields

$$
\begin{aligned}
& I_{e}=I_{d}-I_{s g}-I_{k} \\
& I_{a}=I_{d}+I_{i a}-I_{L}
\end{aligned}
$$

Setting equations 4.39 and 4.40 equal and solving for the beam current gives

$$
I_{b}=\frac{I_{d}\left(V_{d}-V_{c}-2 T_{e V}\right)}{\phi\left(I_{p} U^{+}+I^{*} U^{*}+\left(I_{s g}+I_{k}\right)\left(2 V_{d}-V_{c}+2 \phi\right)+I_{i a}\left(2 T_{e V}+2 \phi\right)+I_{L}\left(V_{d}-V_{c}-2 T_{e V}\right)\right)}
$$

The discharge loss is defined as

$$
\eta_{d}=\frac{I_{d} V_{d}}{I_{b}}
$$

With the beam current defined in equation 4.42 , the discharge loss is

$$
\eta_{d}=\frac{V_{d}\left[\frac{I_{p}}{I_{b}} U^{+}+\frac{I^{*}}{I_{b}} U^{*}+\frac{\left(I_{s g}+I_{k}\right)}{I_{b}}\left(2 V_{d}-V_{c}+2 \phi\right)+\phi+\frac{I_{i a}}{I_{b}}\left(2 T_{e V}+2 \phi\right)+\frac{I_{L}}{I_{b}}\left(V_{d}-V_{c}-2 T_{e V}\right)\right]}{V_{d}-V_{c}-2 T_{e V}}
$$

This equation can be evaluated in parts by the current fractions. The first current fraction is

$$
\frac{I_{p}}{I_{b}}=\frac{2 n_{0} V}{T_{s} A_{s g} v_{B o h m}}\left(\left\langle\sigma_{I} v_{e}\right\rangle+\frac{n_{p}}{n_{e}}\left\langle\sigma_{I} v_{p}\right\rangle\right)
$$

and the second current fraction is similarly

$$
\frac{I^{*}}{I_{b}}=\frac{2 n_{0} V}{T_{s} A_{s g} v_{B o h m}}\left(\left\langle\sigma^{*} v_{e}\right\rangle+\frac{n_{p}}{n_{e}}\left\langle\sigma^{*} v_{p}\right\rangle\right)
$$

The ion current back-flow is very small [12] and the third current fraction can be simplified and written as

$$
\frac{I_{s g}}{I_{b}}=\frac{1-T_{s}}{T_{s}}
$$

The ion current lost to the wall is the Bohm current reduced by the confinement factor $f_{c}$. The calculation of $f_{c}$ is described in equation 4.52 and its constituents. The next current 
fraction is simplified to

$$
\frac{I_{i a}}{I_{b}}=\frac{A_{s a} f_{c}}{T_{s} A_{s g}}
$$

The last current fraction is

$$
\frac{I_{L}}{I_{b}}=\frac{2 n_{p} v_{p} A_{p}}{n e v_{B o h m} A_{s g} T_{s}}
$$

The discharge loss can be rewritten as

$$
\begin{aligned}
\eta_{d}= & V_{d}\left[\frac{I_{p}}{I_{b}} U^{+}+\frac{I^{*}}{I_{b}} U^{*}+\frac{1-T_{s}}{T_{s}}\left(2 V_{d}-V_{c}+2 \phi\right)+\phi+\frac{A_{s a} f_{c}}{T_{s} A_{s g}}\left(2 T_{e V}+2 \phi\right)\right. \\
& \left.+\frac{2 n_{p} v_{p} A_{p}}{n_{e} v_{B o h m} A_{s g} T_{s}}\left(V_{d}-V_{c}-2 T_{e V}\right)\right] /\left(V_{d}-V_{c}-2 T_{e V}\right)
\end{aligned}
$$

and solved for by replacing the first two current fractions with equations 4.45 and 4.46 . With the solution of electron temperature internally determining many of these parameters, solving for the discharge loss becomes easy. As discharge loss is an important factor in the efficiency of an ion thruster, minimizing this value will increase the performance of the ion thruster. A higher screen transparency $T_{s}$, a smaller ion confinement factor $f_{c}$, a smaller anode surface area $A_{s a}$, and lowering the plasma potential $\phi$ will all reduce the discharge loss and increase efficiency.

With the discharge loss now known, a value for the beam current can be calculated from the discharge power the equation

$$
I_{b}=\frac{I_{d} V_{d}}{\eta_{d}}
$$

The beam current is dependent upon the discharge power and the discharge loss. The guess for the discharge current, the input to the minimization routine, is varied until the beam current calculated from the discharge loss is equal to the desired input beam current. The MATLAB function fminbnd is used to determine the discharge current that balances the internally calculated plasma parameters.

Solving for the discharge current required a guess of the discharge current to begin, but also requires a guess for the plasma potential and the confinement factor, as they cannot be solved explicitly without knowing the beam current. The bounded minimization is 
configured to stop and completely converge when the solution for the beam current satisfies the desired input beam current while internally determining the plasma potential and confinement factor which will also satisfy the discharge current equality.

The confinement factor $f_{c}$ is calculated by

$$
f_{c}=\frac{v_{i}}{v_{\text {Bohm }}}
$$

The Bohm velocity, $v_{a}$ or $v_{B o h m}$, is known from 4.5 and the ion velocity $v_{i}[\mathrm{~m} / \mathrm{s}]$ is calculated by

$$
v_{i}=v_{i L}-v_{i R}
$$

with the components

$$
\begin{aligned}
v_{i L} & =0.5 \sqrt{\left(\frac{M_{A M U} d_{\text {cusp }}}{\mu_{e}}\left(1+\mu_{e}^{2} B_{\text {closed }}^{2}-\frac{v_{e i}}{v_{e}}\right)\right)^{2}+4 M_{A M U} T_{e V}} \\
v_{i R} & =\frac{M_{A M U} d_{\text {cusp }}}{2 \mu_{e}}\left(1+\mu_{e}^{2} B_{\text {closed }}^{2}-\frac{v_{e i}}{v_{e}}\right)
\end{aligned}
$$

The plasma potential $\phi[\mathrm{V}]$ can be calculated knowing the discharge current and the confinement factor by

$$
\phi=T_{e V} \cdot \ln \left[\frac{\left(\frac{2 M_{A M U}}{\pi m_{e}}\right)^{1 / 2} \frac{A_{s a}}{A_{s g} T_{s}}}{\frac{I_{d}}{I_{b}}+\frac{A_{s a} f_{c}}{A_{s g} T_{s}}-\frac{2 n_{p} v_{p} A_{p}}{n_{e} v_{a} A_{s g} T_{s}}}\right]
$$

The plasma sheath potential is the difference in potential between the anode and the potential minus the plasma potential drop. In this model, the plasma potential is essentially equal to the anode sheath potential; a graphical representation of the potential distribution from the cathode to the anode can be seen in Figure 4.4. 


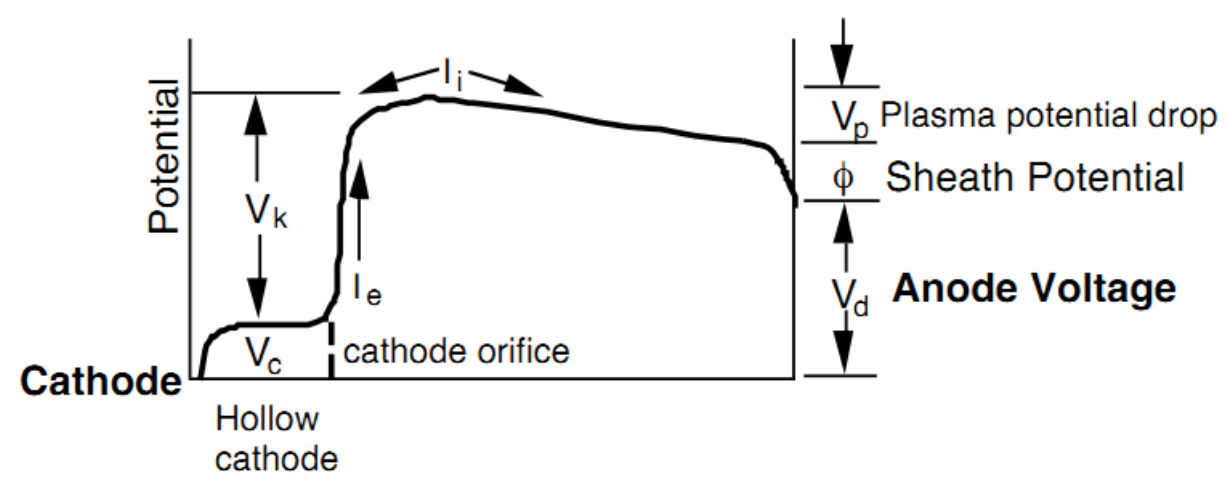

Figure 4.4: Potential Distribution within the Discharge Chamber [15]

The effects of the changing plasma potential is discussed in detail in Section 3.3.2.

Ion Optics Model There are three important values to this optimization that relate directly to the screen and acceleration grid geometry: the clausing factor $\eta_{c}$, the screen ion transparency $T_{s}$, and the screen optical transparency $T_{p}$. As the ion beam passes through the grid system, the beamlets that pass through each hole is compressed and its direction altered due to electrostatic effects. A depiction of this is shown in Figure 4.5.

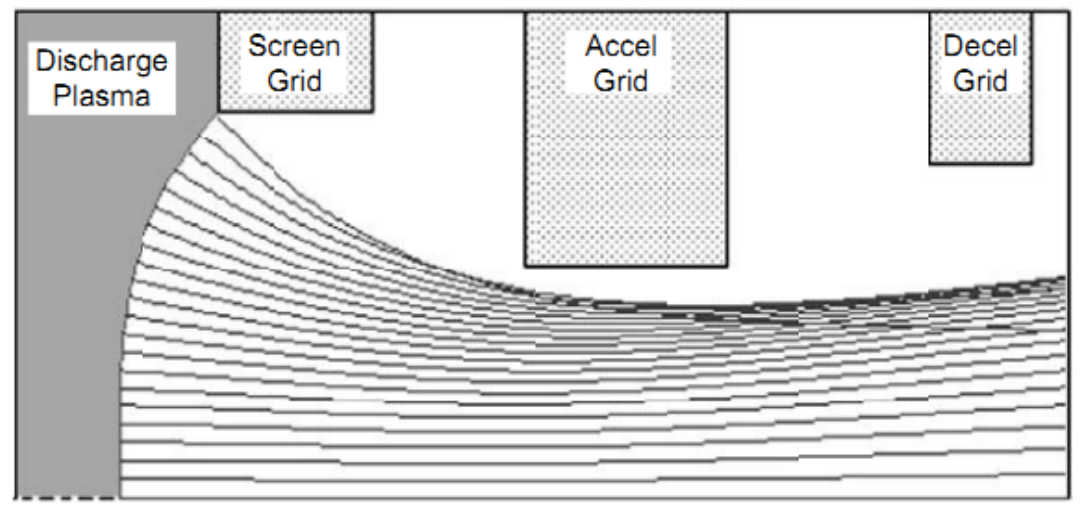

Figure 4.5: Ion Beamlet in a Three-Grid System [12]

JPL has developed a 2D ion optics code often referenced by theses and work completed with JPL $[38,12,1]$. The ion and optical transparency are primarily a function of 
ion density, electron temperature, and beam voltage. In this thesis, estimations of the transparency values from similar thrusters and tests are used [38]. The screen transparency $T_{S}$ is estimated as 0.75 and the optical transparency is estimated as 0.3 .

The clausing factor describes the beam conductance reduction due to the dimensions of the apertures of the thruster. The clausing factor is typically calculated by Monte-Carlo gas flow codes and is well described in [12] in Appendix G. The clausing factor for a set of grids of an ion thruster can be calculated knowing the screen and acceleration grid thickness, the screen and acceleration grid hole radius's, and the spacing between the grids. The geometry of the grids is described in the system configuration section of this report; with a screen and acceleration grid thickness of $0.270 \mathrm{~mm}$, a screen grid hole radius of $0.375 \mathrm{~mm}$, an acceleration hole radius of $0.095 \mathrm{~mm}$, and a grid spacing of $0.330 \mathrm{~mm}$ (Figure 2.20), the clausing factor $\eta_{c}$ was calculated to be 0.4363 .

\subsubsection{Performance Calculations}

The Performance Calculations block of code was the primary section of code within the model and was considered the Objective Function of the optimizer. The goal of the performance calculations was to use the inputs from all the other sub-models to determine the end operation and performance of the thruster. The result of the performance calculations could be varied based on the desired goal for optimization. It was possible to optimize around values such as:

- Total Efficiency $\eta_{T}$

- Discharge Loss $\eta_{d}$

- Thrust $T$

- Specific Impulse Isp

- Power dissipated $P_{d i s}$

- Total Power $P_{0}$ 
The calculation of the performance parameters required a number of inputs similar to the other sub-models. As this is the objective function to the minimization routine, the inputs to this function are the constrained inputs of the entire minimization, listed previously in Section 4.1.1.

From the inputs and the models described above, the quantities of electron temperature, discharge current, and other internally calculated values can be determined and are considered known. The calculation of some of the currents required to determine power and efficiency require knowledge of the resistance of the components, such as the acceleration grid and the keeper assembly. The acceleration grid and the cathode keeper currents can be calculated from the inputs to the performance function.

The beam voltage is the electrostatic potential with which the ion beam is accelerated and is often approximated by the screen grid voltage [12]. Knowing the beam voltage, it is possible to determine the thrust $T[\mathrm{mN}]$ of the ion thruster from

$$
T=1000 \sqrt{\frac{2 M V_{b}}{e}} I_{b} \gamma
$$

where $\gamma$ is a thrust correction factor predominately dependent upon the beam flatness and the ratio of double to single ion production.

$$
\gamma=\cos (\theta) \alpha
$$

The double ion correction factor $\alpha$ is

$$
\alpha=\frac{1+\sqrt{2} \frac{I^{++}}{I^{+}}}{1+\frac{I^{++}}{I^{+}}}
$$

The ion production values are calculated by

$$
\begin{aligned}
I^{+} & =n_{0} n_{e}\left\langle\sigma_{I} v_{e}\right\rangle V e / f_{p}+n_{0} n_{p}\left\langle\sigma_{I} v_{p}\right\rangle V e \\
I^{++} & =n_{e}^{2}\left\langle\sigma_{D} v_{e}\right\rangle V e / f_{p}^{2}+n_{e} n_{p} \sigma_{D} v_{p} V e / f_{p}
\end{aligned}
$$

The values for the reaction rates and cross sections were calculated from electron temperature and primary energy in equations 4.23 and 4.24 . The flatness parameter $f_{p}$ is defined 
as the average current density divided by the peak. For this formulation of the model, the peak density is not calculated; the flatness parameter is approximated to be 0.6 from other thrusters of similar size and configuration $[38,12]$. The beam divergence $\theta$ is also not able to be measured or calculated from this level of code and is estimated to be 10 degrees, a value typical of other ion thrusters with flat grids [12].

The specific impulse $I_{s p}$ is calculated from

$$
I_{s p}=\frac{\gamma \eta_{m}}{g_{0}} \sqrt{\frac{2 e V_{b}}{M}}
$$

The beam power is simply

$$
P_{b}=I_{b} V_{b}
$$

and is used along with the discharge loss value $\eta_{d}$ to determine the electrical efficiency $\eta_{e}$ by the equation

$$
\eta_{e}=\frac{P_{b}}{P_{b}+I_{b} \eta_{d}}
$$

The electrical efficiency is the measure of the beam power over the beam power plus the total power to make the ion beam. The electrical efficiency is used to calculate the input power required to the system

$$
P_{t o t}=\frac{P b}{\eta_{e}}
$$

and the dissipated power is

$$
P_{d i s}=P_{t o t}\left(1-\eta_{e}\right)
$$

The dissipated power is the amount of excess power the thruster must dissipate as heat. This can prove a challenge for small thrusters due to their small external surface area. The excess power is deposited into the thruster components and has the potential of heating the magnets up to the point of permanent demagnetization. Postponing magnet degaussing is a current area of research for these thrusters [30] and excess power reduction was a goal of this thesis. 
The total efficiency $\eta_{T}$ of an ion thruster is defined as the percent of the supplied electrical power that is converted to useful kinetic energy imparted to the spacecraft [12].

$$
\eta_{T}=\gamma^{2} \eta_{m} \eta_{e}
$$

Increasing the total efficiency means there is an increase in the electrical efficiency, thus reducing excess power. With a constant mass utilization efficiency, this leads to the design for a more efficient thruster overall. The total efficiency value was often the focus of the optimization routine.

Another metric for evaluating the performance of ion thrusters is the thrust to power ratio. It is simply the thrust divided by the total power, but can also be written in terms of total efficiency

$$
\frac{T}{P_{t o t}}=\frac{2 \eta_{T}}{g_{0} I_{s p}}
$$

Ion thrusters have very low thrust to power ratio, but they are very efficient; hall thrusters have a higher thrust to power ratio, but are less efficient. For a given input power and total efficiency, there is a trade-off between thrust and specific impulse that can only be improved with more efficient ion thruster designs.

\subsubsection{Power Deposition Sub-Model}

A thermal model was developed that would utilize the results from the performance model to determine the power deposited to components of the thruster. This plasma heating model, developed by Johnathan Van Noord, is derived from the model originally developed for the NSTAR program [28]. The model assumes a 0-D, bulk homogeneous discharge plasma, within which the electron temperature, plasma potential, species densities, and velocities are assumed uniform and constant. The basis of the model relies on the conservation of mass and energy and involves balancing current flow into the components of the discharge chamber. 
The power deposition model is evaluated after the performance calculation model; all of the plasma parameters have been solved previously and are used extensively in solving the power deposition. The results from the performance model also include voltages and currents solved for the various components which will also be used extensively in this submodel. This sub-model attempts to close the loop on the power flow within the discharge chamber and provide results consistent with the previous models.

Ion and Electron Currents A substantial amount of energy injected into the ion thruster is lost to the excitation of neutrals without creating ionization. This model assumes the excitation occurs only from primary electrons from the cathode; the rate at which the neutrals are excited is related to the cathode emission current $I_{e}[\mathrm{~A}]$

$$
I_{e}=I_{d}-I_{s g}
$$

with the discharge current $I_{d}$ and screen current $I_{s g}$ pulled from previous calculations.

The rate at which the excitation of neutrals $I_{n^{*}}$ and ions $I_{+*}$ occur is based on the probability the emitted electrons impact these species with enough energy to cause excitation. The rates are based on the cathode emission current, cross sectional areas of the species, and the mean free path of the electrons $L_{p^{-}}[\mathrm{m}]$

$$
L_{p^{-}}=\frac{1}{n_{0} \sigma_{T}}
$$

and are calculated as

$$
\begin{aligned}
& I_{n^{*}}=I_{e}\left(1-\exp \left(-\sigma_{E} n_{0} L_{p^{-}}\right)\right) \\
& I_{+^{*}}=I_{e}\left(1-\exp \left(-\sigma_{E} n_{i} L_{p^{-}}\right)\right)
\end{aligned}
$$

The keeper ion current $I_{k^{+}}$[A] is calculated through an empirical relationship from the beam current density and is derived from test data from NSTAR and NEXT ion thrusters $[28]$.

$$
I_{k^{+}}=\left(\frac{1.167 I_{b}}{A_{s g}}+0.283\right) A_{k}
$$


Due to the quasi-neutral plasma assumption, the electron current to the keeper $I_{k^{-}}$is set equal to the keeper ion current.

The primary electron current to the anode $I_{a n}^{p^{-}}$is calculated based on a probability of the primary undergoing a collision. If the primaries do not undergo a collision, they are considered lost to the anode wall.

$$
I_{a n}^{p^{-}}=I_{e}\left(\exp \left(-\sigma_{E} n_{0} L_{p^{-}}\right)\right)
$$

The ion current to the anode $I_{a n}^{+}$is calculated by balancing the total ions produced to those lost out of the plasma.

$$
I_{a n}^{+}=I_{p l}^{+}-I_{b}-I_{s g}^{+}-I_{k}^{+}
$$

The Maxwellian electron current to the anode $I_{a n}^{m^{-}}$is determined by another balance of currents within the anode.

$$
I_{a n}^{m^{-}}=I_{d}+I_{a n}^{+}+I_{b}-I_{a n}^{p^{-}}
$$

Power Deposition The average power [W] to ionize an individual beam ion, excite a neutral, or excite an ion ion is found by multiplying the respective currents with the threshold energies [eV for Xenon].

$$
\begin{aligned}
P_{b}^{+} & =I_{b} \cdot U_{+} & U_{+} & =12.13 \mathrm{eV} \\
P_{n}^{*} & =I_{n}^{*} \cdot U_{n^{*}} & U_{n^{*}} & =8.3 \mathrm{eV} \\
P_{i}^{*} & =I_{i}^{*} \cdot U_{i^{*}} & U_{i^{*}} & =11.27 \mathrm{eV}
\end{aligned}
$$

The power added to ionize a beam ion $P_{b}^{+}$leaves the discharge chamber since the energy is used to create the ion. However, the power it takes to excite a neutral or ion is contained within the discharge chamber and the emitted radiation is assumed to be uniformly distributed over the discharge chamber [28]. The calculation of energy to the individual discharge chamber surfaces is determined by a fraction of the surface area of the components to the total discharge chamber surface area. The discharge chamber surface area $A_{d c}$ is the 
sum of the anode area, the screen grid area, and the keeper area exposed to the discharge plasma.

$$
A_{d c}=A_{s a}+A_{s g}+A_{k}
$$

Aside from the excess excitation radiation, the anode is heated by power deposited by the primary electrons, the Maxwellian electrons, and ions. The power from the primary electrons $P_{a n}^{p^{-}}$is the total current of the primary electrons multiplied by the anode work function $\phi_{a n}$ plus the potential difference where the electrons originate in the anode. The work function for the stainless steel anode is estimated as $4.4 \mathrm{eV}$ [17].

$$
P_{a n}^{p^{-}}=I_{a n}^{p^{-}}\left(\phi_{a n}+\left(V_{d}-V_{c}\right)\right)
$$

The power from the Maxwellian electrons $P_{a n}^{m^{-}}$is determined by their temperature and the anode work function

$$
P_{a n}^{m^{-}}=I_{a n}^{m^{-}}\left(\phi_{a n}+5 / 2 T_{e V}\right)
$$

The power deposited from an ion neutralizing on the anode $P_{a n}^{+}$is determined by the anode voltage, ion thermal temperature (assumed to be the neutral temperature $T_{0}$ ), ionization energy, and the anode work function

$$
P_{a n}^{+}=I_{a n}^{+}\left(V_{a}+\frac{5 k T_{0}}{2 e}+U_{+}-\phi_{a n}\right)
$$

The radiated energy fraction $P_{a n}^{*}$ is calculated as

$$
P_{a n}^{*}=\frac{A_{s a}}{A_{d c}}\left(P_{n}^{*}+P_{i}^{*}\right)
$$

The keeper undergoes similar heating to the anode and from the same sources. The power deposited on the keeper from energetic electrons $P_{k}^{-}$is calculated from

$$
P_{k}^{-}=I_{k}^{-}\left(\phi_{k}+5 / 2 T_{e V}\right)
$$

where $\phi_{k}$ is the graphite keeper work function, estimated as $4.83 \mathrm{eV}$ [17]. The power deposited from an ion neutralizing on the keeper $P_{k}^{+}$is dependent on the difference between 
the discharge, anode, and keeper potentials and calculated as

$$
P_{k}^{+}=I_{k}^{+}\left(\left(V_{d}+V_{a}-V_{k}\right)+\frac{5 k T_{0}}{2 e}+U_{+}-\phi_{k}\right)
$$

The radiated energy fraction $P_{k}^{*}$ is calculated as

$$
P_{k}^{*}=\frac{A_{k}}{A_{d c}}\left(P_{n}^{*}+P_{i}^{*}\right)
$$

The screen grid undergoes similar heating to the keeper and the anode and from the same sources. The power deposited on the screen grid from the energetic electrons $P_{s g}^{-}$is calculated from

$$
P_{s g}^{-}=I_{s g}^{-}\left(\phi_{s}+5 / 2 T_{e V}\right)
$$

where $\phi_{s}$ is the work function of the screen grid, estimated as $4.37 \mathrm{eV}$ for molybdenum [17]. The screen grid is also heated by ions drawn to the optics that impact the grid instead of passing through. The power deposited from an ion neutralizing on the screen grid $P_{s g}^{+}$is determined from the difference in voltage between the plasma and the grids and is calculated as

$$
P_{s g}^{+}=I_{s g}^{+}\left(\left(V_{d}+V_{a}\right)+\frac{5 k T_{0}}{2 e}+U_{+}-\phi_{s}\right)
$$

The radiated energy fraction $P_{s g}^{*}$ is calculated as before with the area of the screen reduced by the open area fraction $\Phi_{s o}$ of the screen grid due to the manufactured holes. Knowing the number and diameter of the holes, the open area fraction of the screen grid was determined to be 0.5575 and the radiated energy is calculated as

$$
P_{s g}^{*}=\frac{\left(1-\Phi_{s o}\right) A_{s g}}{A_{d c}}\left(P_{n}^{*}+P_{i}^{*}\right)
$$

The acceleration grid is heated primarily by the charge exchange from ions impacting the acceleration grid and the radiated energy from the excitation as none of the plasma electrons impact the acceleration grid. The power deposited on the acceleration grid from the ions $P_{a} g^{+}$is dependent upon the voltage of the acceleration grid and is calculated as

$$
P_{a g}^{+}=I_{a g}^{+}\left(V_{a g}+\frac{5 k T_{0}}{2 e}+U_{+}-\phi_{a g}\right)
$$


where $\phi_{a g}$ is the work function for the molybdenum acceleration grid. The acceleration grid is partially shielded from the radiated energy from the excitation of ions and neutrals by the screen grid. This shielding results in the deposited power being attenuated by both the screen grid and acceleration grid open fractions. The acceleration grid open fraction $\Phi_{\text {ago }}$ was determined to be 0.0358 and the resulting power deposition from excitation is calculated as

$$
P_{a g}^{*}=\frac{\left(\Phi_{s o}-\Phi_{a g o}\right) A_{s g}}{A_{d c}}\left(P_{n}^{*}+P_{i}^{*}\right)
$$

Determining the power loss from a cathode insert is not a trivial task and often requires a model that includes radiative heat transfer, convection from propellant gas, conduction along the insert and cathode tube, ohmic heating of the cathode, and heating from the plasma [29]. It has been shown that the power loss out of the insert is primarily due to cathode thermal heat loss $Q_{\text {ins }}$. Typical thermal losses out of an insert are around $15 \mathrm{~W}$. This value could be verified through a thermal model of the specific cathode or by measurement during typical operation, but that level of modeling and analysis was not possible and the value of $15 \mathrm{~W}$ was accepted for the thermal heat loss value.

Discharge Power and Losses The components of the power deposition are used to predict the discharge power $P_{d \text {,predict }}$. The summation of the relevant components is

$$
P_{d, \text { predict }}=P_{b}^{+}+P_{n}^{*}+P_{i}^{*}+P_{a n}^{+}+P_{a n}^{m^{-}} \cdot\left(1-\frac{I b}{I_{a n}^{m^{-}}}\right)+P_{a n}^{p^{-}}+P_{k}^{+}+P_{k}^{-}+P_{s g}^{+}+P_{s g}^{-}+Q_{i n s}
$$

It should be noted that while the discharge power includes the power terms to produce beam ions, they do not affect the heating of the thruster.

It has been shown that, generally, the heating of the thruster is highly dependent on the beam current and increases as the beam current increases. At a specific beam current, the heating does not change significantly with the beam voltage. The heating from the Maxwellian electrons associated with the production of the beam ions offset the power due 
to beam ion production; as a result, the total heating of the discharge chamber is approximately equal to the measured discharge power [28].

The following tables demonstrate the component heating predictions from this thermal model for a sample throttle point for MiXI. Also shown are comparisons between the performance model calculations of parameters and the output from the power deposition model.

Table 4.1: Power Deposition Model Sample Throttle Point and Required Outputs

\begin{tabular}{|l|r|c|}
\hline Throttle Parameters & Value & Units \\
\hline Beam Current $I_{b}$ & 0.2 & $\mathrm{~A}$ \\
\hline Mass Utilization $\eta_{m}$ & 0.85 & - \\
\hline Discharge Voltage $V_{d}$ & 24 & $\mathrm{~V}$ \\
\hline Beam Voltage $V_{b}$ & 600 & $\mathrm{~V}$ \\
\hline Performance Model Values & & \\
\hline Electron Temperature $T_{e V}$ & 1.21 & $\mathrm{eV}$ \\
\hline Discharge Current $I_{d}$ & 1.52 & $\mathrm{~A}$ \\
\hline Keeper Voltage $V_{k}$ & 25.99 & $\mathrm{~V}$ \\
\hline Screen Current $I_{s g}$ & 66.7 & $\mathrm{~mA}$ \\
\hline
\end{tabular}

Table 4.1 shows the input throttle setting to the performance model. The performance model values section of the table displays the important relevant parameters needed to solve the power deposition model. 
Table 4.2: Power Deposition Model Output Comparison

\begin{tabular}{|l|r|c|}
\hline Power Deposition Model & Value (W) & Percent Deposited (\%) \\
\hline Ions to Anode $P_{a n}^{+}$ & 8.15 & \\
\hline Primaries to Anode $P_{a n}^{p^{-}}$ & 12.53 & \\
\hline Maxwellian to Anode $P_{a n}^{m^{-}}$ & 7.89 & \\
\hline Keeper Heating from Excitation $P_{a n}^{*}$ & 5.10 & \\
\hline Anode Total & 33.67 & \\
\hline Ions to Keeper $P_{k}^{+}$ & 4.88 & \\
\hline Electrons to Keeper $P_{k}^{-}$ & 0.35 & \\
\hline Keeper Heating from Excitation $P_{k}^{*}$ & 0.19 & \\
\hline Keeper Total & 5.42 & \\
\hline Ions to Screen Grid $P_{s g}^{+}$ & 8.79 & \\
\hline Electrons to Screen Grid $P_{s g}^{-}$ & 0.91 & \\
\hline Screen Heating from Excitation $P_{s g}^{*}$ & 0.43 & \\
\hline Screen Grid Total & 10.13 & \\
\hline Ions to Accelerator Grid $P_{a g}^{+}$ & 8.79 & \\
\hline Accelerator Heating from Excitation $P_{a g}^{*}$ & 0.51 & \\
\hline Accelerator Grid Total & 9.30 & \\
\hline & & \\
\hline Total Discharge Power Predicted & 66.1 & \\
\hline Predicted Discharge Loss & 330.5 & W \\
\hline Predicted Dissipated Power & 58.9 & W/ion \\
\hline & & \\
\hline Perf. Model Discharge Power & 36.5 & W \\
\hline Perf. Model Discharge Loss & 166.1 & \\
\hline Perf. Model Dissipated Power & 33.2 & \\
\hline \% Diff. Discharge Power & & \\
\hline \% Diff. Discharge Loss & 44.7 & \\
\hline Diff. Dissipated Power & & \\
\hline
\end{tabular}


Table 4.2 displays the results from running the power deposition model with the given throttle parameters. The power deposition to the individual components is shown from the different species sources along with a summation per component. The percent deposited value is the component total percent of the predicted dissipated power.

The power deposition model output is useful for determining which specific areas of the thruster are going to be heated the most. The anode draws over $50 \%$ of the total deposited power predicted from the deposition model. It was known prior $[42,30]$ that the anode was heated more intensely than the other components, causing concerns about magnet overheating. With this model, it is possible to see approximately how much power must be dissipated through the anode design. The model also contains the estimation for the total discharge power, the value that would be expected on the discharge power supply during operation. The model also contains the discharge loss, the ratio of the discharge power to the beam current, predicted during operation.

These values are also calculated by the performance model and compared with the output from the power deposition model. The power deposition model predicts a larger amount of discharge power required and a higher discharge loss compared to the performance model output for the same ion beam. This indicates that the power deposition model is predicting a less efficient system requiring more power to produce the beam ions. Both models predict a dissipated power value similar to, and slightly less than, the calculated discharge power. Testing of other thruster designs showed that the dissipated power was approximately equal to the discharge power [28], as was mentioned previously, and both models predict this trend. 


\section{Chapter 5}

\section{MODEL SOLUTIONS}

One vital portion of this thesis was the analysis of the computational model. The goal of the performance model was to have a tool that would analyze the operation of the thruster given a set of inputs to use as a way to characterize the performance of the thruster and to make predictions to it's operation. As will be shown in the following sections, the models were able to complete the analysis desired to provide insight to the operation and also to characterize the performance of the thruster.

\subsection{Performance Model Output}

The performance model operates on a set of inputs, iterates on a solution, and internally solves all the relevant plasma parameters. The inputs are listed in Section 4.1.1 and include the throttle point of the thruster. Other inputs to the computational model include those relevant to the magnetic configuration of the thruster. A sample input to the computational model for the baseline configuration is shown in Table 5.1. 
Table 5.1: Computational Model Sample Throttle Point

\begin{tabular}{|l|r|c|}
\hline Throttle Parameters & Value & Units \\
\hline Beam Current $I_{b}$ & 0.2 & $\mathrm{~A}$ \\
\hline Mass Utilization $\eta_{m}$ & 0.85 & - \\
\hline Discharge Voltage $V_{d}$ & 24 & $\mathrm{~V}$ \\
\hline Beam Voltage $V_{b}$ & 600 & $\mathrm{~V}$ \\
\hline Magnetics Inputs & & \\
\hline Closed Loop Field Strength & 368 & Gauss \\
\hline Base Ring Cusp Strength & 2207 & Gauss \\
\hline Upper Anode Ring Cusp Strength & 3011 & Gauss \\
\hline Lower Anode Ring Cusp Strength & 9.2 & mm \\
\hline Anode Cusp Spacing & & Gauss \\
\hline
\end{tabular}

The performance model iterates and calculates the plasma parameters based on the methods and equations presented in Section 4. The resulting output from the throttle setting and magnetics configuration is shown in Table 5.2. 
Table 5.2: Computational Model Sample Throttle Point

\begin{tabular}{|c|c|c|}
\hline Performance Model Output & Value & Units \\
\hline \multicolumn{3}{|l|}{ Plasma Characteristics } \\
\hline Neutral Plasma Density $n_{0}$ & $3.366 \times 10^{19}$ & $n / m^{3}$ \\
\hline Maxwellian Density $n_{e}$ & $5.660 \times 10^{18}$ & $n / m^{3}$ \\
\hline Primary Density $n_{p}$ & $2.183 \times 10^{16}$ & $n / m^{3}$ \\
\hline Electron Temperature $T_{e V}$ & 0.949 & $\mathrm{eV}$ \\
\hline Discharge Loss $\eta_{d}$ & 166 & eV/ion \\
\hline Plasma Potential $\phi$ & 3.24 & $\mathrm{~V}$ \\
\hline Probability of Collision & 61.5 & $\%$ \\
\hline Confinement Factor $f_{c}$ & 0.062 & - \\
\hline \multicolumn{3}{|l|}{ Operational Values } \\
\hline Discharge Current $I_{d}$ & 1.55 & A \\
\hline Mass Flow Rate $Q_{i n}$ & 2.79 & SCCM \\
\hline Keeper Voltage $V_{k}$ & 25.2 & $\mathrm{~V}$ \\
\hline Keeper Current $I_{k}$ & 89.2 & $\mathrm{~mA}$ \\
\hline Screen Current $I_{s}$ & 66.7 & $\mathrm{~mA}$ \\
\hline \multicolumn{3}{|l|}{ Performance Characteristics } \\
\hline Specific Impulse $I_{s p}$ & 2513 & S \\
\hline Thrust $T$ & 7.890 & $\mathrm{mN}$ \\
\hline Total Power $P_{t o t}$ & 153.2 & $\mathrm{~W}$ \\
\hline Thrust to Power Ratio & 0.052 & - \\
\hline Dissipated Power $P_{d i s s}$ & 33.2 & $\mathrm{~W}$ \\
\hline Electrical Efficiency $\eta_{e}$ & 78.4 & $\%$ \\
\hline Total Efficiency $\eta_{T}$ & 63.5 & $\%$ \\
\hline
\end{tabular}


There are a few things of note about the output of the performance model. First and foremost, the plasma characteristics are reasonable values for this throttle level and the MiXI design [38, 22]. The electron species densities are split and indicate that the majority of ionization of particles is going to occur due to the Maxwellian electrons in the plasma as they are 100 times more dense.

The electron temperature is a rather low value at this throttle point, but it is reasonable. Measurements of the plasma distribution within other $3 \mathrm{~cm}$ discharge chambers [22] demonstrate that there is a wide electron temperature distribution within the discharge chamber. As the code in this performance model is a $0-\mathrm{D}$ averaged solution, it is reasonable to assume the electron temperature might be an averaged value across the entire discharge chamber, resulting in a lower value for $T_{e V}$. The discharge loss is calculated at a level reasonable for other ion thrusters [12] and indicates that the thruster should be able to maintain the discharge plasma with a reasonable discharge power. Further analysis of the discharge loss will be explored in Section 5.2.

The operational values in this table show the set points of the mass flow fuel input device and the discharge power supply required to attain this throttle level. The screen and keeper power supplies would be set to their respective potentials. The keeper and screen current are internally determined and not controlled externally; the output here demonstrates that the values are not unreasonable, however the keeper current displayed is only going to be a fraction of what is actually read on the power supply as the estimation for the current does not hold as true for MiXI.

The performance characteristics display the end value results of this thruster configuration and throttle setting. This set point has a reasonable specific impulse for an ion thruster, but has a high relative thrust when comparing to other thrusters of this class $[38,19]$. The cause for this is not known as the calculation to determine thrust is very straight forward, however, it was not possible to measure thrust in any way during testing to verify this value. 
The power and efficiency values indicate that there is a large amount of power required to operate the thruster at this throttle point and configuration. The performance model indicates that the thruster will require $153.2 \mathrm{~W}$ of power to operate, which is more than other thrusters of this class have required $[38,19]$. There is an electrical efficiency of $78.4 \%$ and the amount of power that must be dissipated is $33.2 \mathrm{~W}$, indicating that excessive heating of the thruster may be an issue. The total efficiency is $63.5 \%$ and is a measure of what percent of the supplied electrical power that is converted to usable kinetic energy is imparted to the spacecraft. While the efficiency values are reasonable and good for a thruster of this size, it is hoped there will be marked improvements to this value arising from changing the throttle settings and magnetic configurations.

\subsection{Performance Curves}

Typically, discharge chamber behavior is characterized by performance curves [12] comparing an output of the thruster to one or more of the controlled inputs. With regards to the models, this involves iterating through spans of input parameters and analyzing the resultant values. In thruster operation, the discharge current and total gas flow can be controlled by the operator and varied to produce a constant beam current and discharge voltage with a specific mass utilization value. The performance model works similarly in that is specifies the input beam current, discharge voltage, and mass utilization, then determines the discharge current that will produce the internally consistent plasma parameters. With all the plasma parameters known, the efficiency terms for the throttle point are calculated.

Performance curves are typically a graph of discharge loss over a span of mass utilization efficiencies with a set beam current and discharge voltage [12]. It is desirable to have a low discharge loss to increase electrical efficiency and to have a high mass utilization to increase fuel efficiency. The shape of these performance curves is also important as they provide indication towards optimal thruster design: a thruster design with flatter discharge 
performance can provide high mass utilization at reasonably low discharge losses [15]. Figure 5.1 shows a sample performance curve for an ideal $30 \mathrm{~cm}$ thruster example.

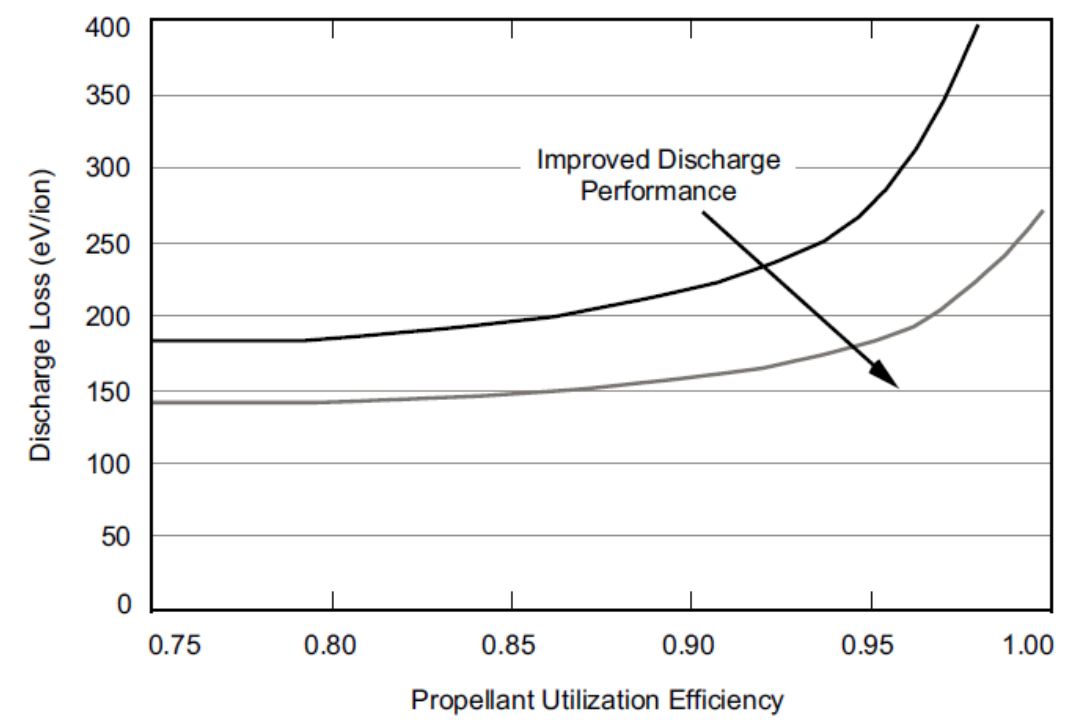

Figure 5.1: Performance Curve Example of an Ideal Thruster [12]

This curve shows the flat characteristics of an ideal ion thruster design, maintaining low discharge loss over a large span of mass utilization efficiencies, thus allowing for numerous throttle points with similar total efficiencies. It is expected that the discharge loss will exponentially increase as the mass utilization approaches one. Thrusters are normally operated near the 'knee' of the curve as to attain high mass utilization while maintaining low discharge loss [12]. As the graph shows, discharge performance is improved as the trace is moved downward. This shift in the graph can be achieved through changing the beam current of the throttle point or by changing the discharge voltage of the design.

The performance model was analyzed and similar performance curves were produced. These curves characterize the MiXI thruster, allow for predictions to be made about the thruster performance, and can be compared to performance curves from other thruster designs. Figure 5.2 displays three performance curves, showing spans from the output of the 
models over the mass utilization efficiency at three different beam currents typical to MiXI operation. The discharge voltage was held constant at $24 \mathrm{~V}$.

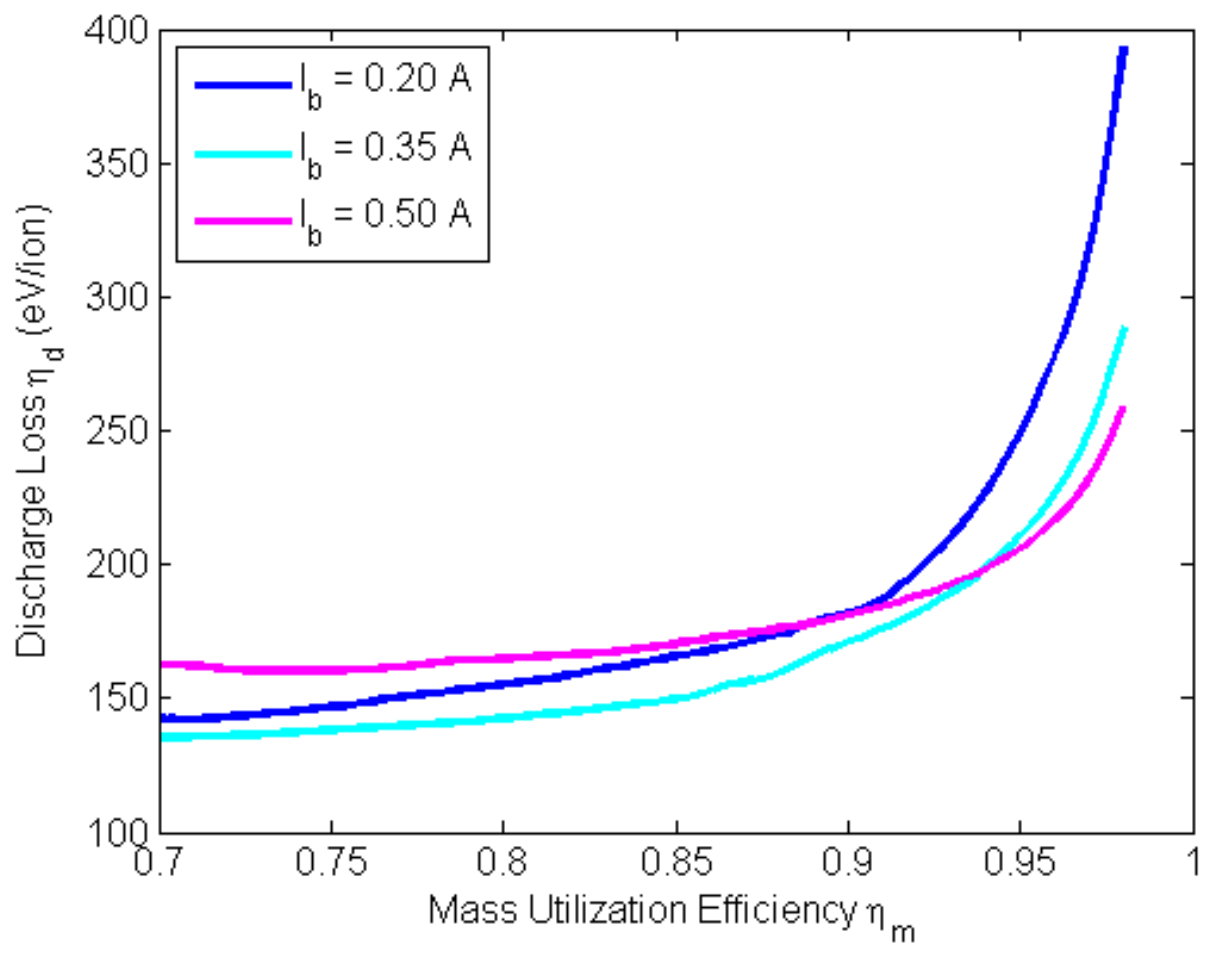

Figure 5.2: Performance Model Output: Discharge Loss vs. Mass Utilization Efficiency with Varying Beam Current; Discharge Voltage $=24 \mathrm{~V}$

From the figure, it is immediately apparent that the MiXI thruster has performance curves similar to those of the idealized $30 \mathrm{~cm}$ thruster. The model also shows the discharge loss to be within a similar range when compared to the values from the idealized thruster, indicating that the MiXI thruster should be able to perform well and be able to provide a low current ion beam without requiring disproportionately large amounts of power.

The multiple traces on the graph indicate the performance curve at different beam currents within the thruster's operational capabilities. Changing the beam current demonstrates the different performance characteristics when various beam currents are desired. At higher beam currents, the thruster has a higher discharge loss than at lower currents for low mass utilizations, but has a more flat profile, indicating a more optimized design capable of op- 
erating efficiently at a wider range of mass utilization efficiencies. The two lower current traces are similar at low mass efficiencies, but deviate at the higher values, following the trend with the higher beam current trace having a lower discharge loss. The knee of these curves lies between 0.92 and $0.97 \eta_{m}$ for the three traces and indicates that the desired throttle point would exist at a mass utilization within that range.

The performance model was analyzed again and similar performance curves were created that altered the discharge voltage while keeping the beam current constant. The resulting performance curves are show in Figure 5.3. The beam current was held at a constant $0.2 \mathrm{~A}$ for these performance curves.

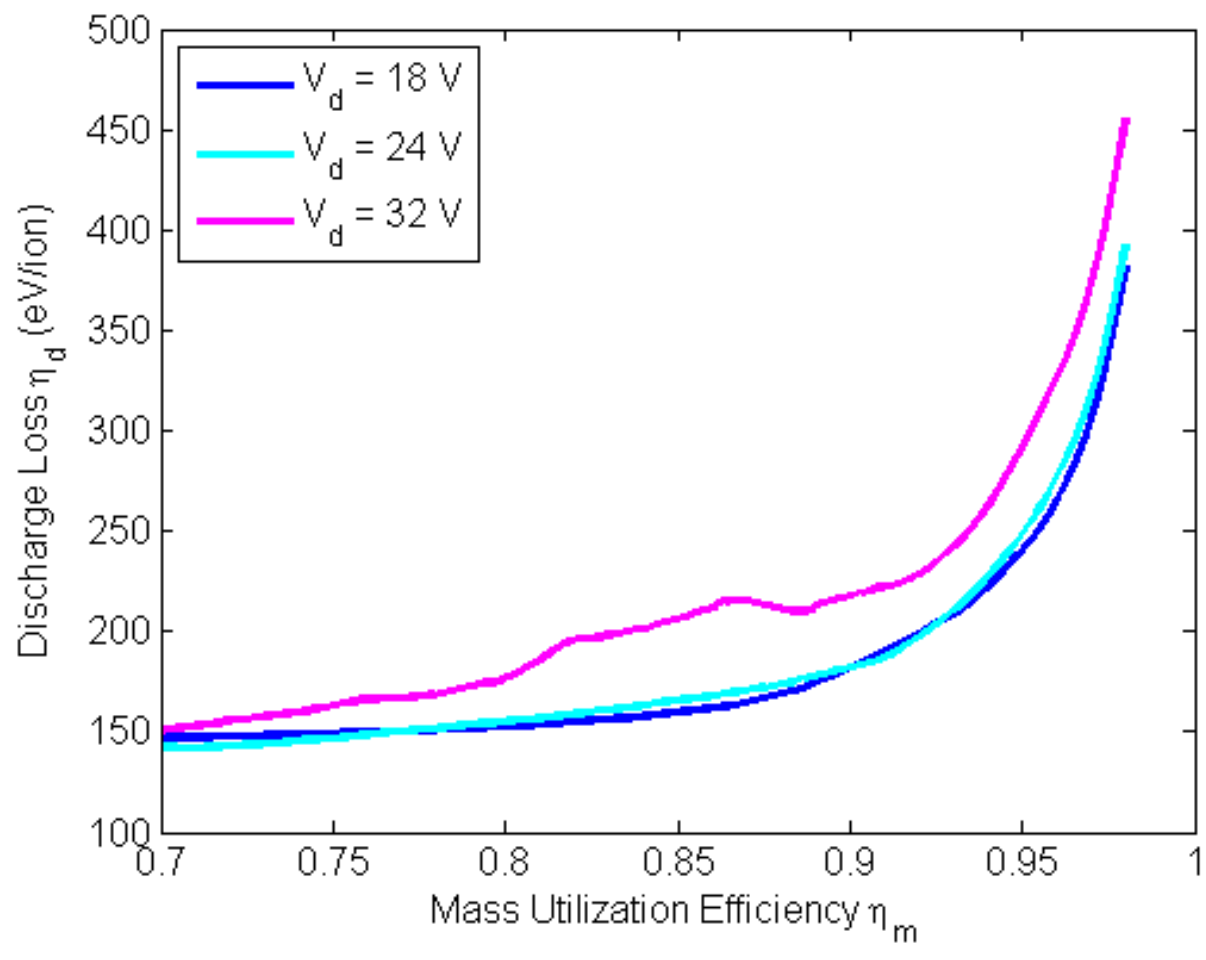

Figure 5.3: Performance Model Output: Discharge Loss vs. Mass Utilization Efficiency with Varying Discharge Voltage; Beam Current $=0.2 \mathrm{~A}$

These performance curves all show results one would expect for the MiXI thruster based on the previous analysis varying the discharge current. This graph shows there is variation in the performance based on the discharge voltage but that the variation is not 
nearly as significant or influential as the changes in beam current are. The knees of all three curves in this figure indicate the desired throttle level would have 0.95 mass utilization efficiency.

Knowing the beam current is going to significantly impact the discharge loss, a different type of performance curve was generated that calculated the discharge loss versus the beam current explicitly. Figure 5.4 shows four performance curves with varying levels of mass utilization efficiencies. The discharge voltage in these curves is held constant at $24 \mathrm{~V}$.

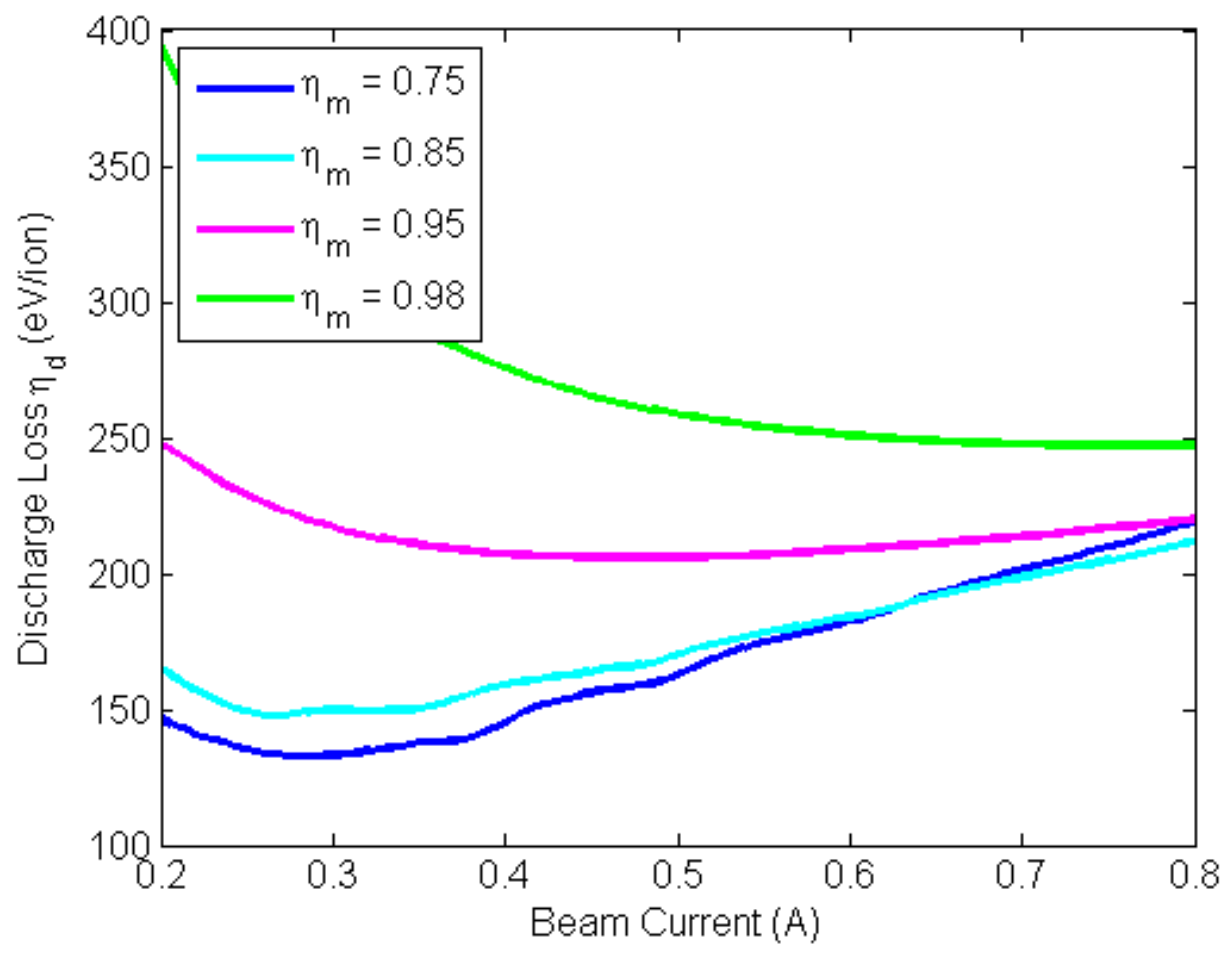

Figure 5.4: Performance Model Output: Discharge Loss vs. Beam Current with Varying Mass Utilization Efficiency; Discharge Voltage $=24 \mathrm{~V}$

These curves are enlightening in that they show what the most efficient beam current is for the MiXI thruster in terms of discharge loss for different mass utilization levels. The curves are similar for the low mass utilization values of 0.75 and 0.85 ; the most efficient beam current is around $0.275 \mathrm{~A}$. At the higher mass utilization values, the performance is much different and the discharge loss is much higher. The most efficient beam current 
at 0.95 mass utilization is $0.48 \mathrm{~A}$ and has a discharge loss of $210 \mathrm{eV} /$ ion. At 0.98 mass utilization, the minimum discharge loss is about $245 \mathrm{eV} /$ ion at a discharge current over $0.8 \mathrm{~A}$ and outside the operational range of the MiXI thruster. At the same $0.275 \mathrm{~A}$ beam current optimal for the low mass utilization cases, the high mass utilization throttle points have much greater discharge losses.

The performance model was analyzed again by varying the beam current, but keeping the mass utilization at a fixed value and demonstrating change due to the discharge voltage. Figure 5.5 shows three performance curves with varying discharge voltages and a constant mass utilization of 0.85 .

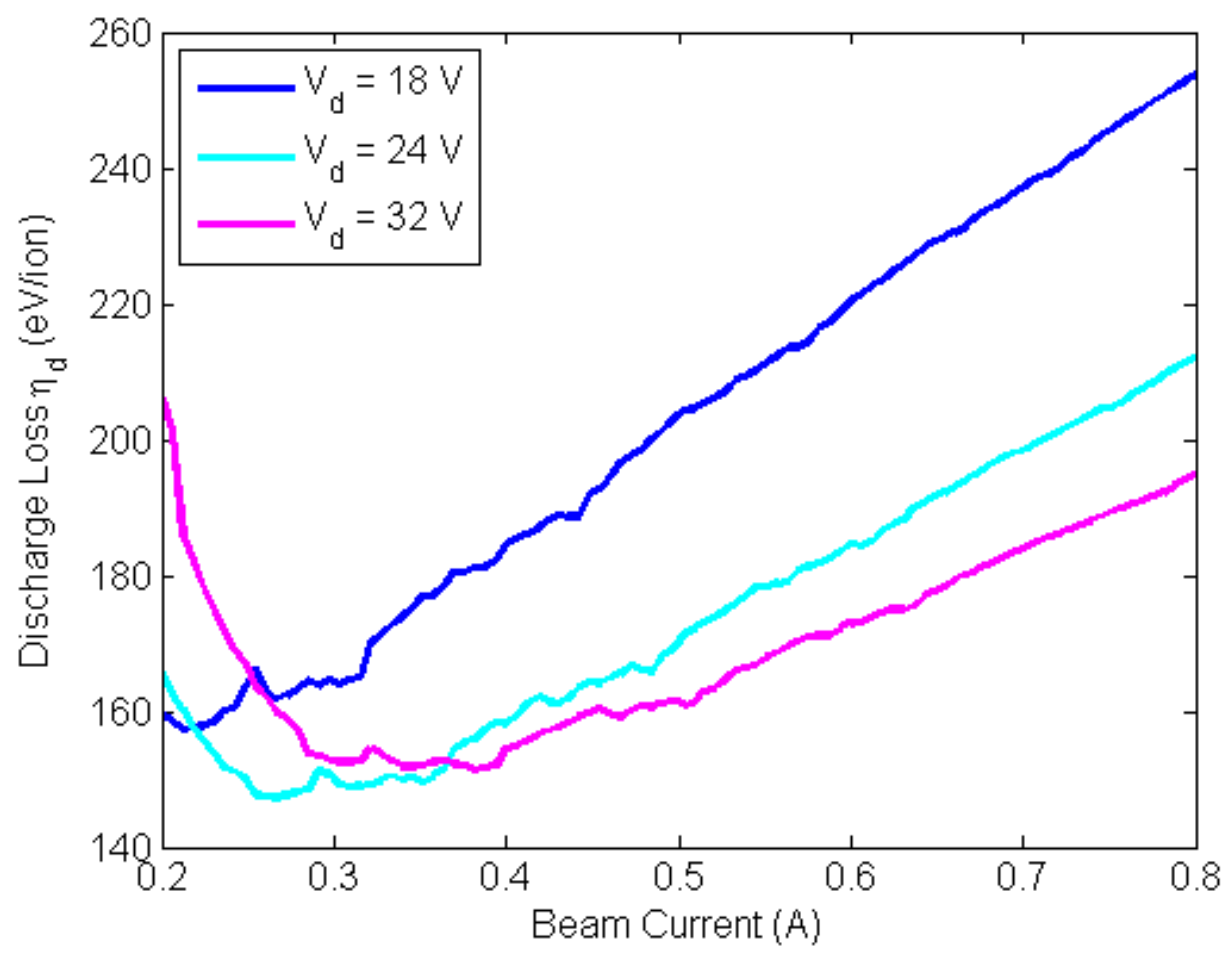

Figure 5.5: Performance Model Output: Discharge Loss vs. Beam Current with Varying Discharge Voltage; Mass Utilization = 0.85

This figure again shows there is variation to the performance curves as a result of changing the discharge voltage. The trend in this graph shows there is a measure of increased performance when increasing the discharge voltage: the curves are lower and have less 
overall discharge loss with increasing voltage. At $32 \mathrm{~V}$, the optimal beam current is around $0.34 \mathrm{~A}$ with a discharge loss of $152 \mathrm{eV} /$ ion. At $24 \mathrm{~V}$, the optimal beam current is at $0.28 \mathrm{~A}$ with a discharge loss of $155 \mathrm{eV} /$ ion. At $18 \mathrm{~V}$, there is a larger change and the optimal beam current is at $0.22 \mathrm{~A}$ with a discharge loss of $177 \mathrm{eV} / \mathrm{ion}$. This graph indicates that it should be possible to run the thruster efficiently at higher discharge voltages with larger beam currents, but MiXI is still capable of running efficient at very low power settings.

To demonstrate the idea of changing the discharge voltage further, Figures 5.6 and 5.7 present the discharge loss while varying the discharge voltage. Figure 5.6 shows the performance curves over a span of discharge voltages with a constant mass utilization of 0.85 . In this analysis, the beam current is varied between curves.

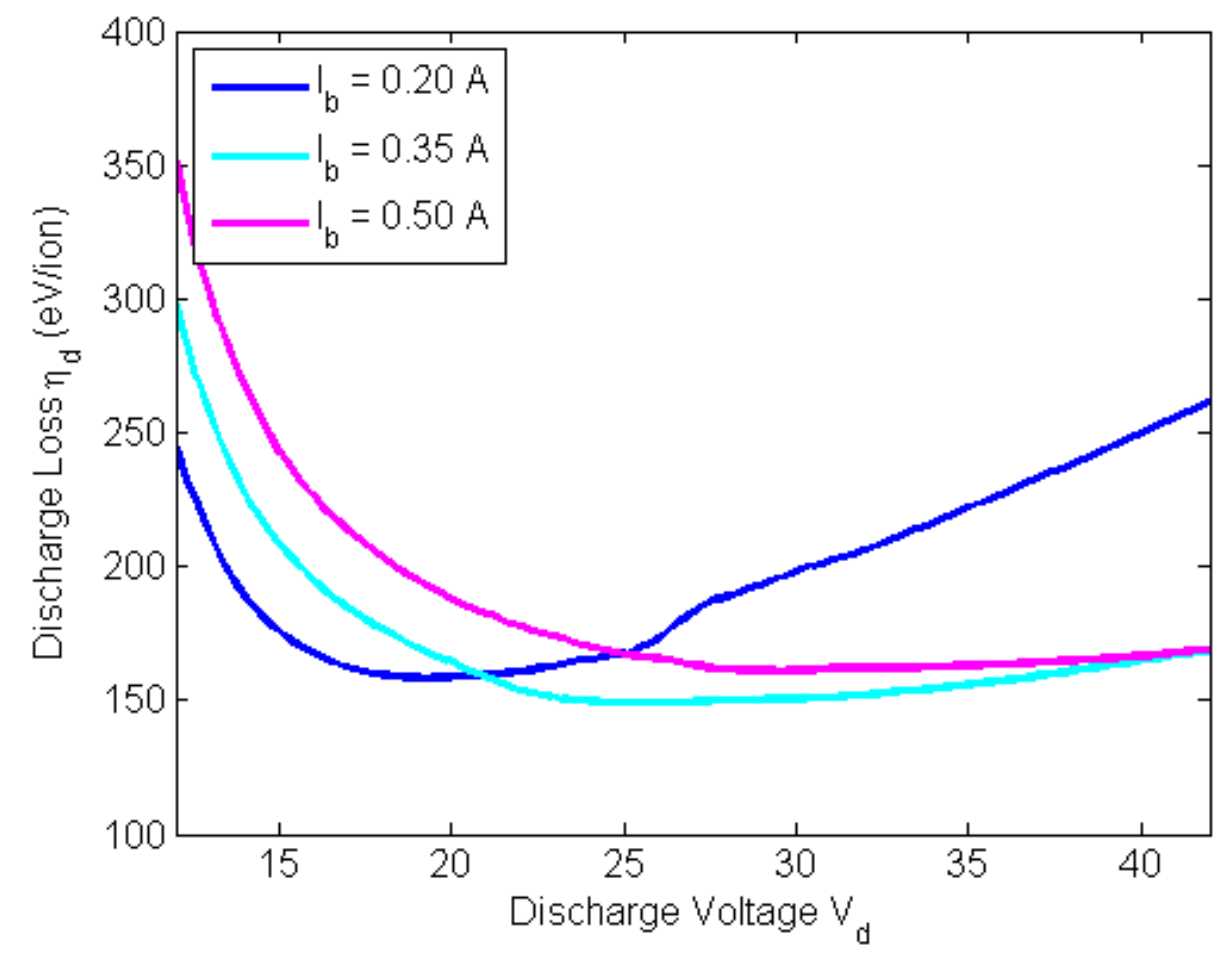

Figure 5.6: Performance Model Output: Discharge Loss vs. Discharge Voltage with Varying Beam Current; Mass Utilization = 0.85

From the figure, it is apparent that the discharge loss has an important dependence upon the discharge voltage over a range of beam currents. At the lower discharge voltages, the 
lower beam current is more efficient. At higher discharge voltages, the $0.35 \mathrm{~A}$ and $0.5 \mathrm{~A}$ beam current cases are more efficient and the $0.2 \mathrm{~A}$ case has a much higher discharge loss. The $0.5 \mathrm{~A}$ beam current case will eventually be more efficient than the $0.35 \mathrm{~A}$ case, but only at discharge voltages outside of the range capable of this thruster.

Typically, increasing the discharge current will decrease the discharge loss [12]. The performance model calculates many internal parameters self-consistently; for specific throttle points holding the beam current constant, the computational model determines the discharge current will decrease with discharge voltage. This change of multiple parameters as the discharge voltage increases leads to an increasing discharge loss past the minimum.

Figure 5.7 shows four performance curves over the span of discharge voltages, varying mass utilization efficiency and maintaining a constant beam current of $0.2 \mathrm{~A}$.

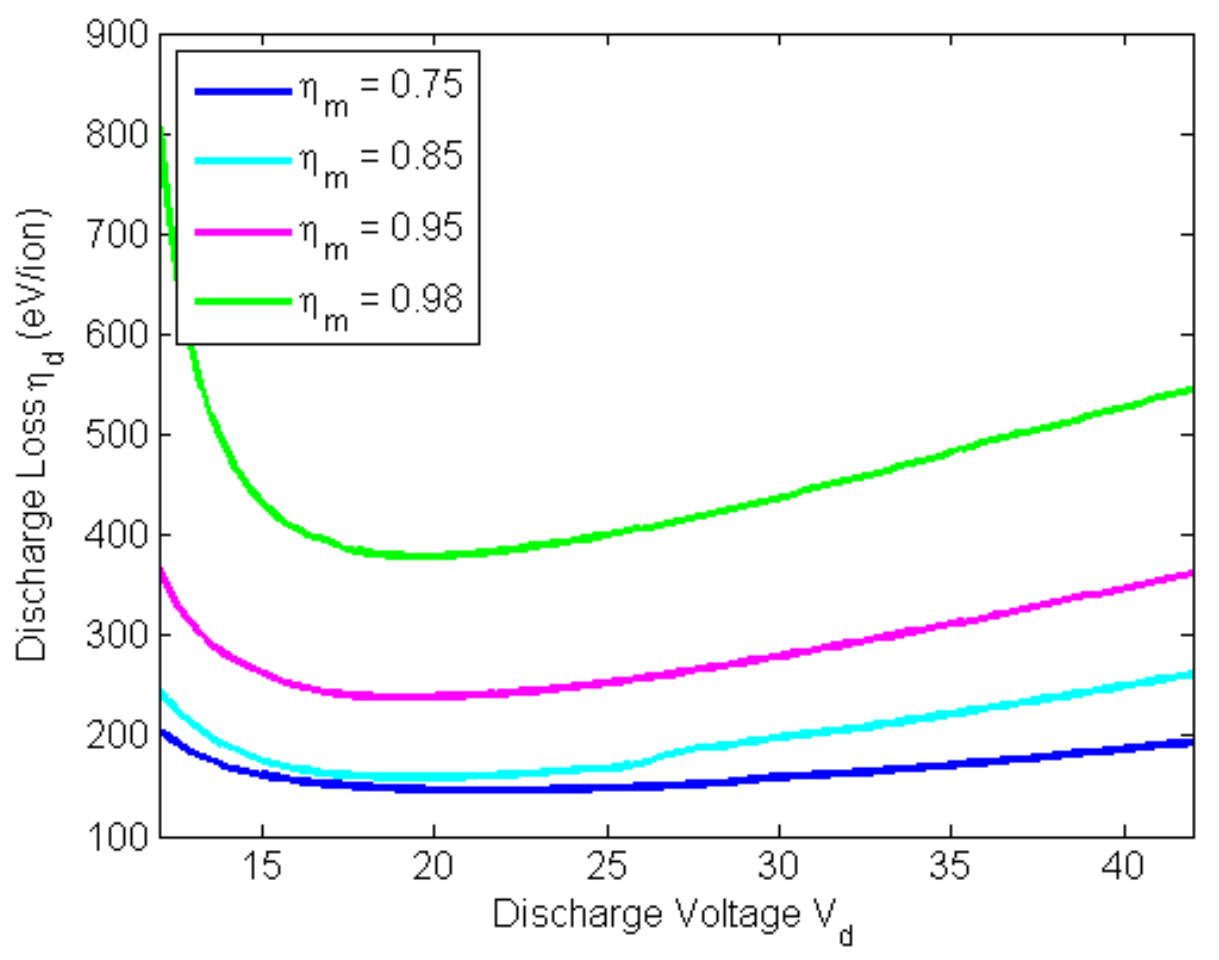

Figure 5.7: Performance Model Output: Discharge Loss vs. Discharge Voltage with Varying Mass Utilization Efficiency; Beam Current $=0.2 \mathrm{~A}$ 
It is easy to see that a smaller value for mass utilization will always result in a lower discharge loss. The two lower mass utilization cases are similar in shape and magnitude of the discharge loss, having a minimum near $24 \mathrm{~V}$ of about $150-160 \mathrm{eV} /$ ion. The higher mass utilization efficiency curves have almost two to four times the discharge loss at the same discharge voltages. While the minimum of the curves occurs at a lower discharge voltage, the discharge loss becomes prohibitively large.

The trends of these performance curves are to provide the user a way to qualitatively judge the impact on performance altering the throttle parameters will have. While it is desirable to have a high mass utilization efficiency value, the discharge loss associated with that value can become exceedingly large and detrimental to the operation of the thruster.

\subsection{Thruster Optimization}

As was discussed previously in Section 4, the computational model was built in such a way to run within the optimizer fmincon in MATLAB. The optimizer changes the input values to the performance model and converges on a solution based on the chosen objective. In this work, the optimizer was set to converge on the best solution based on total efficiency $\eta_{T}$. Tables 5.1 and 5.2 displayed the initial throttle value and resultant output of the performance model for a single throttle level. The total efficiency for that setup was only $36.8 \%$ and it was obvious that there was room for improvement.

Table 5.3 shows the input throttle point values and their bounds for all the optimization runs. 
Table 5.3: Computational Model Throttle Point Bounds for Optimization

\begin{tabular}{|l|r|r|r|c|}
\hline Throttle Parameters & \multicolumn{1}{|l|}{$\begin{array}{l}\text { Initial } \\
\text { Value }\end{array}$} & \multicolumn{1}{l|}{$\begin{array}{l}\text { Lower } \\
\text { Bounds }\end{array}$} & \multicolumn{1}{l|}{$\begin{array}{l}\text { Upper } \\
\text { Bounds }\end{array}$} & Units \\
\hline Beam Current $I_{b}$ & 0.2 & 0.1 & 0.5 & $\mathrm{~A}$ \\
\hline Mass Utilization $\eta_{m}$ & 0.85 & 0.7 & 0.95 & - \\
\hline Discharge Voltage $V_{d}$ & 24 & 15 & 42 & $\mathrm{~V}$ \\
\hline Beam Voltage $V_{b}$ & 600 & 200 & 700 & $\mathrm{~V}$ \\
\hline
\end{tabular}

These bounds were chosen because either the test equipment MiXI was to be run on couldn't perform at levels outside the bounds, or because the data from the previous discharge curves indicated that operating outside the specified bounds would prove greatly inefficient.

Figure 5.8 is a plot of convergence for the baseline configuration when running through the optimizer.

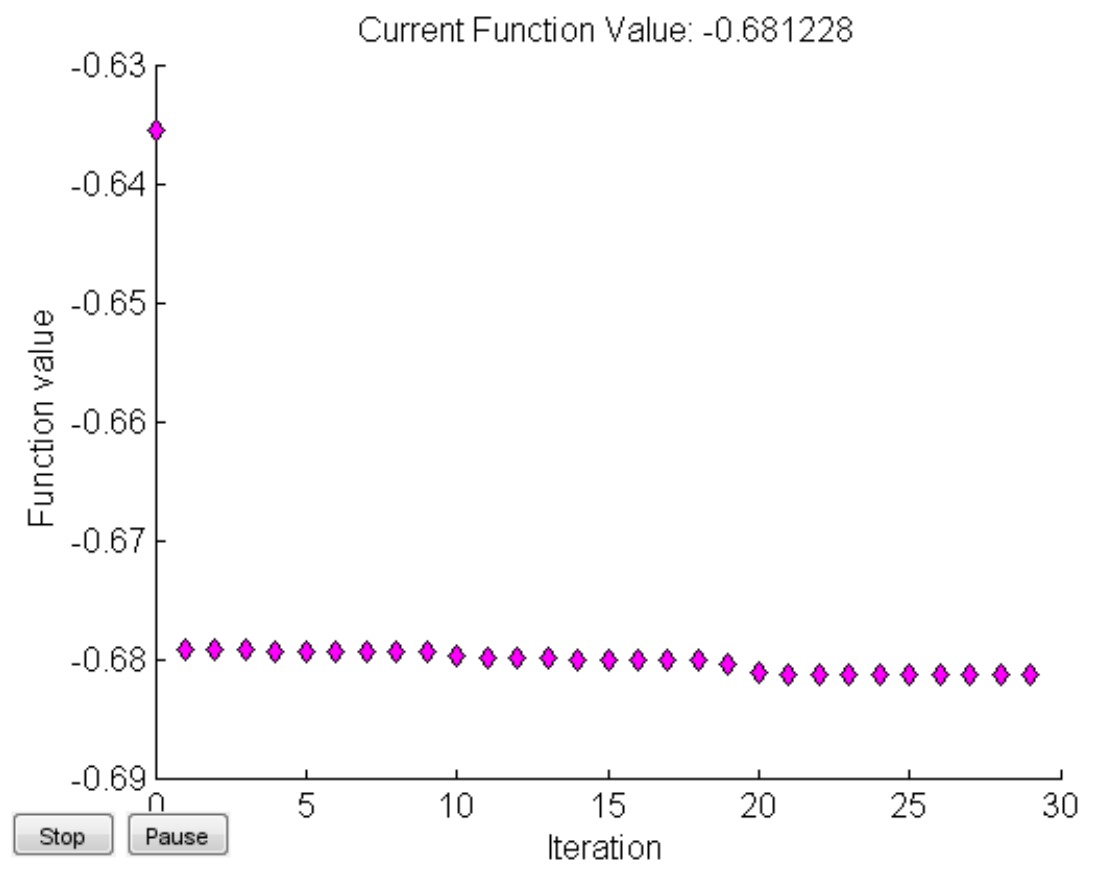

Figure 5.8: MATLAB Optimization Result of Baseline Configuration, Maximizing Total Efficiency 
From the figure, it is easy to see the quick decrease followed by the slower refinement in the function value over the iteration steps. The optimization required 29 iterations and 414 function evaluations to solve. The displayed value is negative because the optimizer can only minimize; the absolute value of the function value is the total efficiency $\eta_{T}$ of the thruster. The initial point has the same efficiency as the data displayed in Table 5.2 of $63.5 \%$. The final point has a value of $68.1 \%$, indicating an increase in total efficiency of the thruster.

Tables 5.4 and 5.5 display the optimized throttle levels and resultant output from the performance model.

Table 5.4: Throttle Level Result of Optimization on the Baseline Configuration, Maximizing Total Efficiency

\begin{tabular}{|l|r|c|}
\hline Throttle Parameters & Value & Units \\
\hline Beam Current $I_{b}$ & 0.46 & $\mathrm{~A}$ \\
\hline Mass Utilization $\eta_{m}$ & 0.93 & - \\
\hline Discharge Voltage $V_{d}$ & 27.7 & $\mathrm{~V}$ \\
\hline Beam Voltage $V_{b}$ & 700 & $\mathrm{~V}$ \\
\hline
\end{tabular}

The optimizer determined that to achieve the highest total efficiency value, it would be required to run the thruster at a throttle level with $0.46 \mathrm{~A}$ beam current, 0.93 mass utilization, and $700 \mathrm{~V}$ beam voltage. Through analyzing the equations, it would be expected to desire as large of a beam power as possible, as that is a dominating term for the electrical efficiency, and thus the total efficiency. However, as the trends from the previous section would indicate, increasing the beam current too much will cause inefficiencies in creating the ion beam and result in a lower total efficiency. 
Table 5.5: Performance Model Output of Baseline Optimization, Maximizing Total Efficiency

\begin{tabular}{|c|c|c|}
\hline Performance Model Output & Value & Units \\
\hline \multicolumn{3}{|l|}{ Plasma Characteristics } \\
\hline Electron Temperature $T_{e V}$ & 2.31 & $\mathrm{eV}$ \\
\hline Discharge Loss $\eta_{d}$ & 191 & $\mathrm{eV} /$ ion \\
\hline Plasma Potential $\phi$ & 9.06 & $\mathrm{~V}$ \\
\hline Probability of Collision & 62.3 & $\%$ \\
\hline \multicolumn{3}{|l|}{ Operational Values } \\
\hline Discharge Current $I_{d}$ & 1.78 & A \\
\hline Mass Flow Rate $Q_{i n}$ & 6.40 & $\mathrm{SCCM}$ \\
\hline Keeper Voltage $V_{k}$ & 31.4 & $\mathrm{~V}$ \\
\hline Keeper Current $I_{k}$ & 204.5 & $\mathrm{~mA}$ \\
\hline Screen Current $I_{S}$ & 153.0 & $\mathrm{~mA}$ \\
\hline \multicolumn{3}{|l|}{ Performance Characteristics } \\
\hline Specific Impulse $I_{s p}$ & 2778 & $\mathrm{~s}$ \\
\hline Thrust $T$ & 18.2 & $\mathrm{mN}$ \\
\hline Total Power $P_{t o t}$ & 363.9 & $\mathrm{~W}$ \\
\hline Thrust to Power Ratio & 0.050 & - \\
\hline Dissipated Power $P_{d i s s}$ & 87.9 & $\mathrm{~W}$ \\
\hline Electrical Efficiency $\eta_{e}$ & 75.9 & $\%$ \\
\hline Total Efficiency $\eta_{T}$ & 68.1 & $\%$ \\
\hline
\end{tabular}

There are several important things to note about the output of the "optimal" throttle level, the first being the total power and the power dissipated. The total power required to operate MiXI at this throttle level becomes quite large compared to the initial throttle point, 
requiring over twice as much power to operate. With the additional power required, the dissipated power increases, meaning the small thruster is going to overheat much quicker. The keeper current is the result of the approximation from other thruster's data and may not hold true when used for MiXI; while the value indicated here is low, the power supply will require much higher current to operate at the desired voltage. The thruster also requires a much higher mass flow rate to create the required beam current; on a small thruster for small scale applications, the propellant utilization may become an influential factor.

Analysis of the gradient of the output indicates the mass utilization value impacts the performance and the total efficiency greatest. Small changes to the mass utilization value at the optimum point reduce the total efficiency; decreasing the mass utilization will decrease the discharge loss and the total power, and increasing the mass utilization will increase the discharge loss and total power, while decreasing the total efficiency of the at this operating point. Reducing the mass utilization by a value of 0.02 reduces the total efficiency to $67.8 \%$ and decreases the required power to $358.2 \mathrm{~W}$. The trade between total efficiency and required power can be realized using multi-objective optimization or implementing a cost function as the objective of fmincon.

To further explore the high power problem, it was possible to reconfigure the optimizer to determine the optimum throttle setting that would yield the smallest dissipated power within the bounds of the optimization. Figure 5.9 demonstrates the convergence of the optimizer minimizing the dissipated power. 


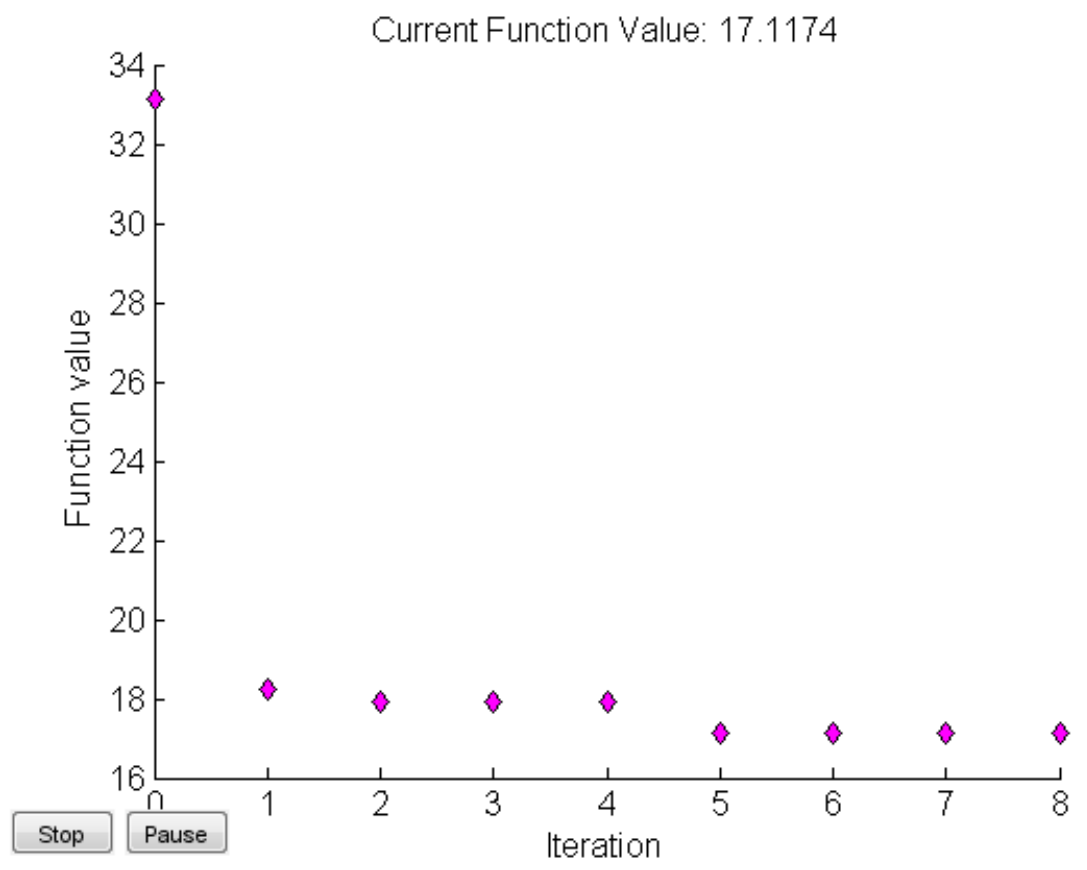

Figure 5.9: MATLAB Optimization Result of Baseline Configuration, Minimizing Power Dissipated

The graph shows the decrease in dissipated power occurring over 8 iterations and 108 function evaluations. The initial dissipated power value was $33.2 \mathrm{~W}$ and the final value was 17.1W. Tables 5.6 and 5.7 show the throttle values and the resultant model outputs for this optimization run.

Table 5.6: Throttle Level Result of Optimization on the Baseline Configuration, Minimizing Dissipated Power

\begin{tabular}{|l|r|c|}
\hline Throttle Parameters & \multicolumn{1}{|c|}{ Value } & Units \\
\hline Beam Current $I_{b}$ & 0.10 & $\mathrm{~A}$ \\
\hline Mass Utilization $\eta_{m}$ & 0.70 & - \\
\hline Discharge Voltage $V_{d}$ & 23.32 & $\mathrm{~V}$ \\
\hline Beam Voltage $V_{b}$ & 200 & $\mathrm{~V}$ \\
\hline
\end{tabular}


The optimizer runs the beam current, mass utilization, beam voltage, and keeper voltage to their lower limits in order to reduce the excess power. The discharge voltage is chosen to create the lowest discharge loss possible at this configuration.

Table 5.7: Performance Model Output of Baseline Optimization, Minimizing Dissipated Power

\begin{tabular}{|c|c|c|}
\hline Performance Model Output & Value & Units \\
\hline \multicolumn{3}{|l|}{ Plasma Characteristics } \\
\hline Electron Temperature $T_{e V}$ & 0.77 & $\mathrm{eV}$ \\
\hline Discharge Loss $\eta_{d}$ & 167 & $\mathrm{eV} /$ ion \\
\hline Plasma Potential $\phi$ & 2.25 & $\mathrm{~V}$ \\
\hline Probability of Collision & 75.9 & $\%$ \\
\hline \multicolumn{3}{|l|}{ Operational Values } \\
\hline Discharge Current $I_{d}$ & 0.97 & A \\
\hline Mass Flow Rate $Q_{i n}$ & 1.43 & $\mathrm{SCCM}$ \\
\hline Keeper Voltage $V_{k}$ & 23.5 & $\mathrm{~V}$ \\
\hline Keeper Current $I_{k}$ & 45.7 & $\mathrm{~mA}$ \\
\hline Screen Current $I_{S}$ & 34.1 & $\mathrm{~mA}$ \\
\hline \multicolumn{3}{|l|}{ Performance Characteristics } \\
\hline Specific Impulse $I_{s p}$ & 1202 & s \\
\hline Thrust $T$ & 2.34 & $\mathrm{mN}$ \\
\hline Total Power $P_{t o t}$ & 37.6 & $\mathrm{~W}$ \\
\hline Thrust to Power Ratio & 0.062 & - \\
\hline Dissipated Power $P_{d i s s}$ & 17.1 & $\mathrm{~W}$ \\
\hline Electrical Efficiency $\eta_{e}$ & 54.5 & $\%$ \\
\hline Total Efficiency $\eta_{T}$ & 36.7 & $\%$ \\
\hline
\end{tabular}


One interesting thing to note about the optimization for lowest power dissipated is the very similar discharge loss value to the other minimization. The values are very similar and indicate that, regardless of the goal, it is always important to maintain a low discharge loss value, otherwise more significant problems arise. The specific impulse and thrust of this throttle point are very low compared to the initial throttle setting and the optimization around total efficiency. It should also be noted that the electrical efficiency is still comparable to the input throttle point but the total efficiency is quite small; while this configuration has very low dissipated power, it is primarily because the total input power is much smaller.

These two optimizations demonstrate the capability of the model code and its ability to determine the performance of the thruster at a wide range of throttle values. The optimization portion is powerful in that the solver can determine the optimum throttle point for any design requirement and for any number of potential inputs. As was mentioned earlier, a mission specific cost function could be created and the optimizer would be able to determine the best geometry, magnetic configuration, and throttle point for the mission.

\subsubsection{Magnetic Field Optimization}

Another goal of this thesis was to identify changes to the performance of MiXI due to changes in the magnetic field structure. As was discussed earlier, there were a total of 48 configurations prepared and analyzed. The performance model code has inputs alloted for the magnetic field configuration and the optimizer is able to input any of the prepared configurations. All of the magnetic field configurations were analyzed and run through the optimizer. The results were dissected in an effort to identify changes in the total efficiency value as well as in other performance parameters.

The least efficient configuration was $2 o 31 t$ with a total efficiency of $41.4 \%$ after optimization. The most efficient configuration analyzed was $4 i 13 s$ with a total efficiency of $69.3 \%$ after optimization. There were five magnetic configurations that had total efficiency 
values greater than the baseline configuration. The top 14 most efficient configurations used the spread anode magnet rings, and the top 4 configurations used the base magnetic ring located inwards. The poorest performing configurations were those with the anode magnet rings top biased. The optimized throttle level for the most efficient configuration is shown in Table 5.8 .

Table 5.8: Throttle Level Result of Optimization on the $4 i 13 s$ Configuration, Maximizing Total Efficiency

\begin{tabular}{|l|r|c|}
\hline Throttle Parameters & \multicolumn{1}{|c|}{ Value } & Units \\
\hline Beam Current $I_{b}$ & 0.48 & A \\
\hline Mass Utilization $\eta_{m}$ & 0.94 & - \\
\hline Discharge Voltage $V_{d}$ & 24.9 & V \\
\hline Beam Voltage $V_{b}$ & 700 & V \\
\hline
\end{tabular}

The beam current, mass utilization, and beam voltage were all run to their limits within the optimization, similar to the optimization performed on the baseline configuration. The discharge voltage is at a level that provides the lowest discharge loss with the desired beam current and mass utilization value. Table 5.7 displays the model output of the same configuration at the optimal throttle level. 
Table 5.9: Performance Model Output of $4 i 13 s$ Optimization, Maximizing Total Efficiency

\begin{tabular}{|l|r|c|}
\hline Performance Model Output & \multicolumn{1}{|c|}{ Value } & Units \\
\hline Plasma Characteristics & & \\
\hline Electron Temperature $T_{e V}$ & 2.30 & $\mathrm{eV}$ \\
\hline Discharge Loss $\eta_{d}$ & 183.7 & $\mathrm{eV} / \mathrm{ion}$ \\
\hline Plasma Potential $\phi$ & 9.73 & $\mathrm{~V}$ \\
\hline Probability of Collision & 53.3 & $\%$ \\
\hline Operational Values & & \\
\hline Discharge Current $I_{d}$ & 1.83 & $\mathrm{~A}$ \\
\hline Mass Flow Rate $Q_{i n}$ & 6.69 & $\mathrm{SCCM}$ \\
\hline Keeper Voltage $V_{k}$ & 33.30 & $\mathrm{~V}$ \\
\hline Keeper Current $I_{k}$ & 214.0 & $\mathrm{~mA}$ \\
\hline Screen Current $I_{s}$ & 160.0 & $\mathrm{~mA}$ \\
\hline Performance Characteristics & & \\
\hline Specific Impulse $I_{s p}$ & 2799 & $\mathrm{~s}$ \\
\hline Thrust $T$ & 19.1 & $\mathrm{mN}$ \\
\hline Total Power $P_{\text {tot }}$ & 376.4 & $\mathrm{~W}$ \\
\hline Thrust to Power Ratio & 0.051 & - \\
\hline Dissipated Power $P_{\text {diss }}$ & 88.2 & $\mathrm{~W}$ \\
\hline Electrical Efficiency $\eta_{e}$ & $\mathbf{6 9 . 4}$ & $\%$ \\
\hline Total Efficiency $\eta_{T}$ & & \\
\hline & & \\
\hline
\end{tabular}

As with the other optimization runs that maximize on total efficiency, there are problems and limitations that arise. The thruster requires a large amount of power and, while electrically efficient, still has a large dissipated power term. The plasma potential term is 
also very large, indicating that the plasma, while stable, has a large sheath and may be over-confined. The keeper current is also very small; as mentioned before, this value arises from a power balance within the discharge chamber and is based on assumptions that may be causing errors. Other larger thrusters often have a much larger discharge current value, but the plasma discharge sub-model determines the current balance as indicated.

Figure 5.10 displays the magnetic field analysis for this configuration.
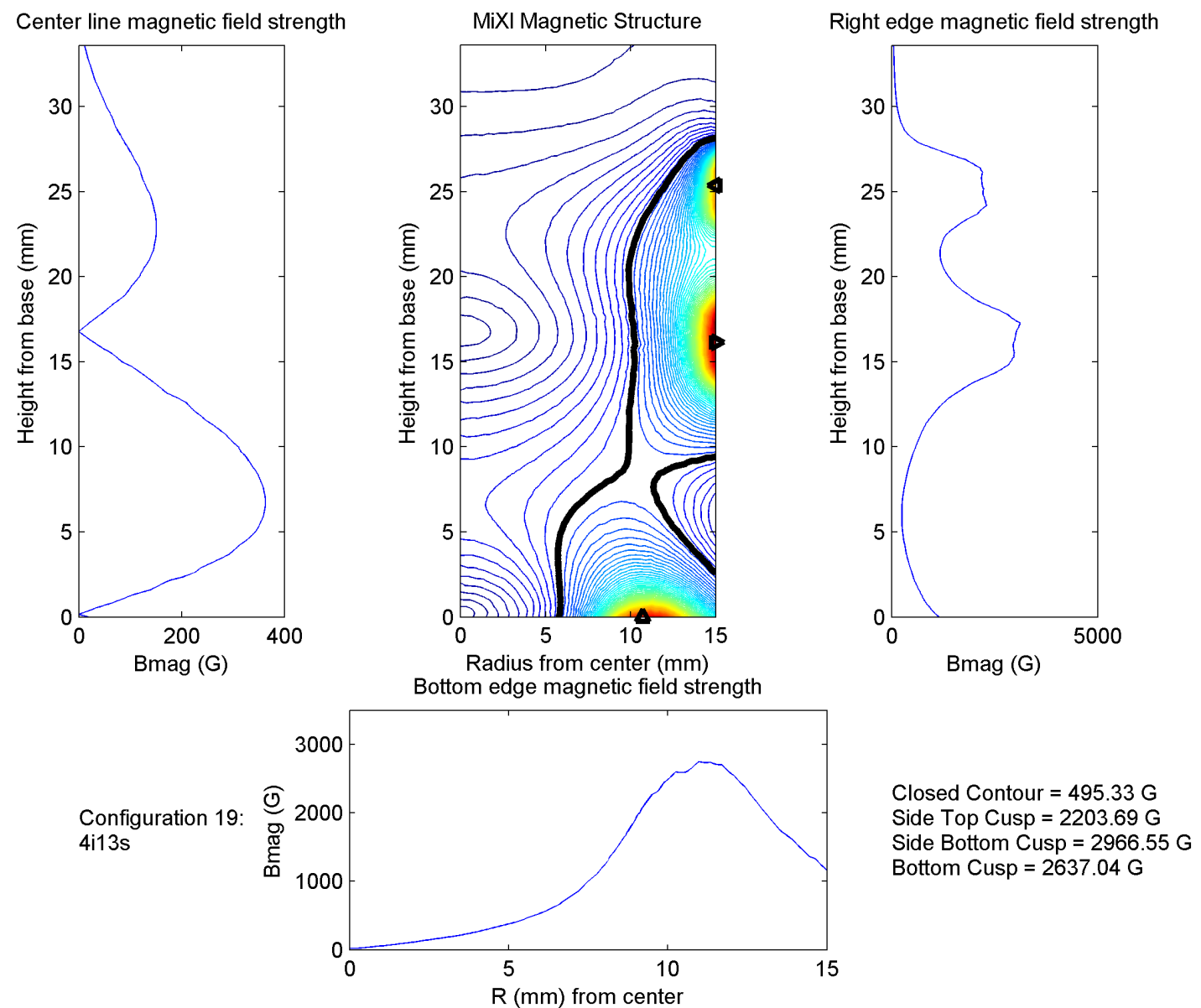

Figure 5.10: Magnetic Field Analysis of Configuration 4i13s

The magnetic field analysis shows that the optimal configuration has a very high closed loop magnetic field strength but that the loop does not create the full $360^{\circ}$ closed contour. The centerline trace also indicates that there is a significant secondary peak. Both of these 
indicate that there are still changes to the magnetic configuration that would potentially alter the performance further, however a higher order model would be needed to fully understand the effects. 


\section{Chapter 6}

\section{TESTING}

Operational testing of the hollow cathode and ion thruster was conducted to determine the validity of the results from the computational model. The baseline configuration was tested extensively in two operating modes. Three additional magnetic configurations of the

thruster were set up and run. In addition to recording data from the power supplies, a Langmuir probe was used to determine the plasma parameters of the beam and the discharge plasma.

All testing was performed in Cal Poly's Space Environments Laboratory. This section presents the testing facilities, test apparatus setup, and testing procedures.

\subsection{Experimental Apparatus}

The following sections present the test facility and the setup of the test equipment.

\subsubsection{Vacuum Chamber}

Validation testing for this project was conducted in the Minimum Atmospheric eXperimentation (MAX) chamber. The chamber has an 18 inch diameter, 12 inch tall Sci-Tec glass cylindrical jar that encompasses the test platform. The removable bell jar has two rubber O-rings on the top and bottom and is sealed by a pneumatic lid. The test apparatus, with installed thruster, is shown in Figure 6.1. 


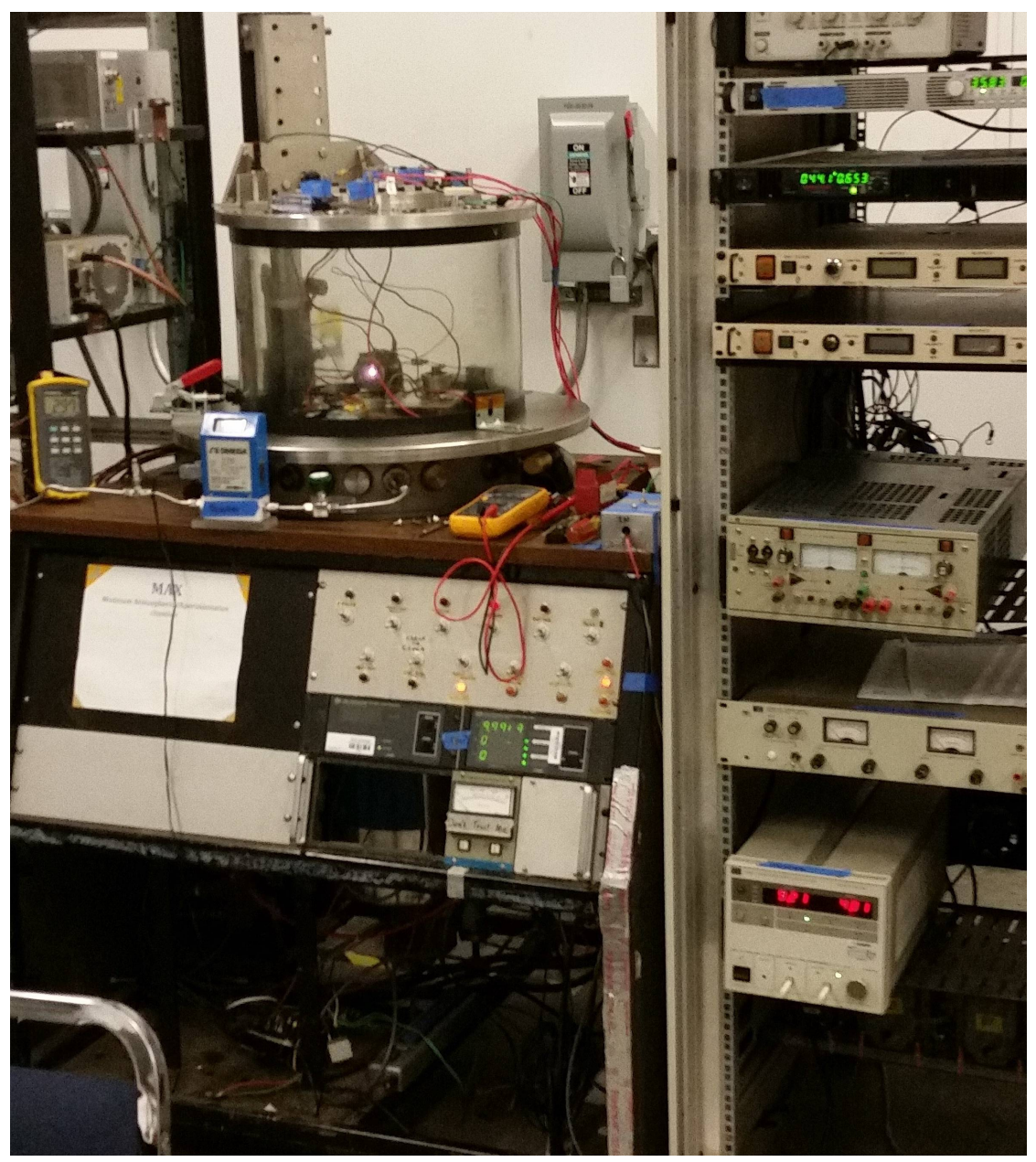

Figure 6.1: Minimum Atmospheric eXperimentation Chamber (MAX), Cal Poly Space Environments Laboratory

The MAX chamber utilizes two vacuum pumps for different levels of vacuum. A Varian 600DS Scroll Pump is used to bring the base pressure of the chamber to as low as $5 \times 10^{-3}$ Torr. This is not low enough for thruster operation, so a series of pneumatic valves are used to connect the chamber to a CTI CryoTorr 10 cryogenic pump, which utilizes a CTI Cryogenics 1020R compressor. With the assistance of the cryogenic pump, the base pressure of the chamber can reach as low as $5.2 \times 10^{-7}$ Torr. An annotated schematic of the chamber and the pump system is shown in Figure 6.2. 


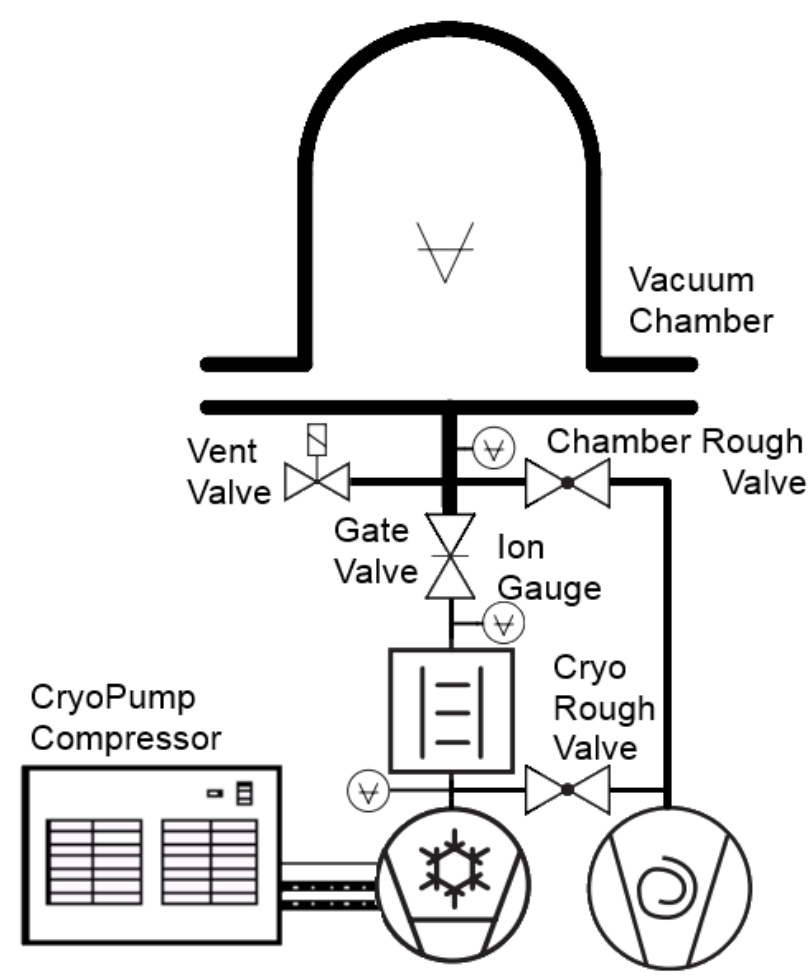

Figure 6.2: Schematic of MAX Vacuum Chamber and Pump Systems

The chamber and cryogenic pump line pressures are read through convectron gauges and an ion gauge. A Granville-Phillips 316 vacuum gauge controller reads the chamber and the cryogenic pump line pressure down to $1 \times 10^{-3}$ Torr. When the pressure drops below this level, a Granville-Phillips 330 Ion Gauge controller and a GP 274 Glass Ion Gauge Tube reads the pressure of the cryogenic pump line and the vacuum chamber when the gate valve is opened. The locations of these are also shown in the vacuum chamber schematic. These gauges are calibrated to read pressures of $N_{2}$ gas and a pressure conversion factor had to be used to determine the actual pressure of the chamber when the dominant gas was something other than $N_{2}$. 


\subsubsection{Electrical Configuration}

The electrical system for an ion thruster requires five to seven power supplies operating simultaneously. This means there are a number of electrical systems that must be connected through the vacuum chamber and isolated from all other components. The MAX chamber has 26 threaded feedthrough locations located radially along the bottom of the vacuum chamber, 4 conductive rod electrical feedthroughs on the lid of the chamber, 2 BNC terminals centrally isolated also on the lid, and 2 fully isolated BNC terminals on the bottom of the chamber.

The hollow cathode requires two power supplies and two feedthroughs: one for the heater and one for the keeper. The negative terminal of these power supplies are connected to a common third feedthrough. The heater supply is used to heat the cathode insert to the required temperature to begin electron emission. The keeper supply is used to initiate plasma production, 'strike' the cathode, and to reduce high energy ion bombardment during normal operation. In some cases, the heater power supply can be re-purposed as the anode/discharge power supply once the cathode is struck as the cathode should be in a self heating state during operation [12]. This was not possible for the cathode in this experiment as the heater needed to be maintained at a low power setting to maintain insert temperature and electron production.

The strike voltage of the keeper is determined based on the Child-Langmuir Law [12]. For electrons, the current density $J\left[A / \mathrm{m}^{2}\right]$ is written as

$$
J=\frac{4 \varepsilon_{0}}{9} \sqrt{\frac{2 e}{m_{e}}} \frac{V^{3 / 2}}{d^{2}}
$$

This equation describes the current density that flows through empty space over a distance $d$. The current density increased proportional to the voltage to the $3 / 2$ power divided by the distance squared. The exact current density required to strike the cathode depends on the temperature of the insert and the amount and type of propellant gas flowing through the 
cathode. The voltage required to achieve that current density is dependent on the design and spacing of the cathode orifice plate and the keeper. The cathode may require as much as $1 \mathrm{kV}$ to strike. The setup for this cathode required the use of a high voltage power supply connected in parallel with the keeper supply to strike the cathode. The voltage and current flow is controlled using diodes and the electrical schematic is shown in Figure 6.3.

The discharge chamber, also referenced as the anode, requires a power supply and another feed through. The negative terminal is connected to the same common terminal as the heater and keeper supplies. The discharge power supply applies a positive voltage to the anode that will attract electrons emitted from the cathode and the plasma. The hollow cathode is capable of running in a stand alone mode of operation utilizing the three aforementioned power supplies and four feedthroughs in a simple configuration for testing the cathode in discharge mode [22]. When operating in this stand alone configuration, the common node of the heater, keeper, and anode is connected to earth ground. An electrical schematic and component diagram are shown in Figure 6.3.

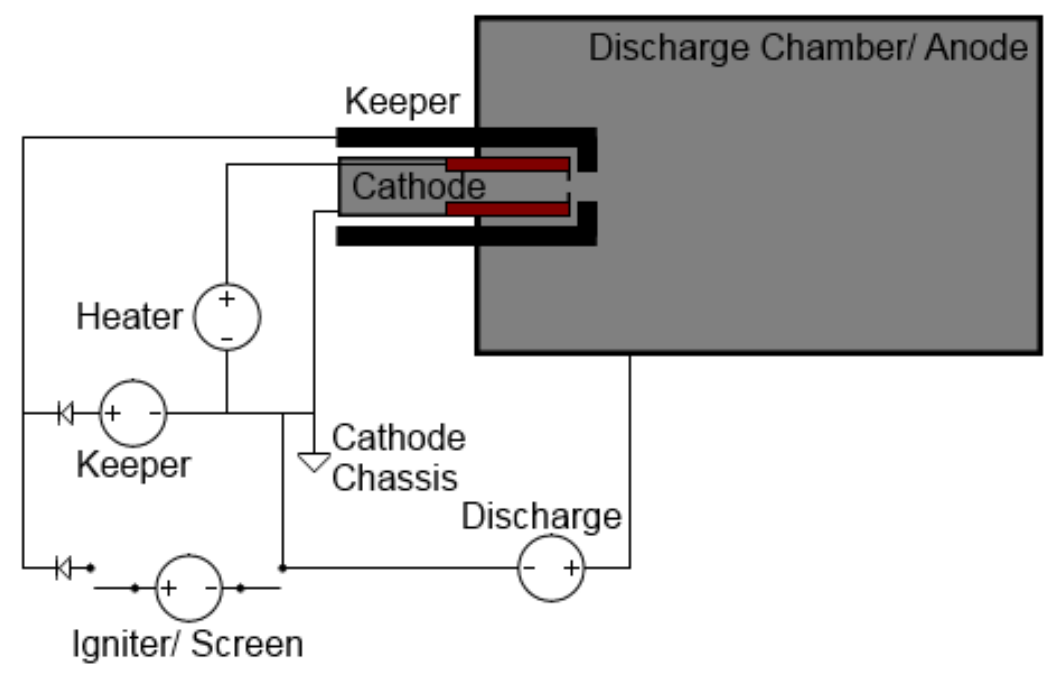

Figure 6.3: Hollow Cathode Stand Alone Schematic and Electrical Diagram 
The screen grid is connected to the common node of the heater, the keeper, the discharge power supply, and to ground. The screen power supply is the same power supply used to strike the cathode but switched onto a different circuit. The accelerator power supply is connected with a negative reference between ground and the accelerator grid through a $5 \mathrm{k}$ ohm resistor. The resistors in line with the screen and acceleration grid supplies are to attenuate any arcs that may occur during the striking of the cathode and to protect the power supplies. The screen supply raises the potential of the entire thruster relative to ground and the acceleration grid. A depiction of the relative potentials of the system is shown in Figure 6.4 .

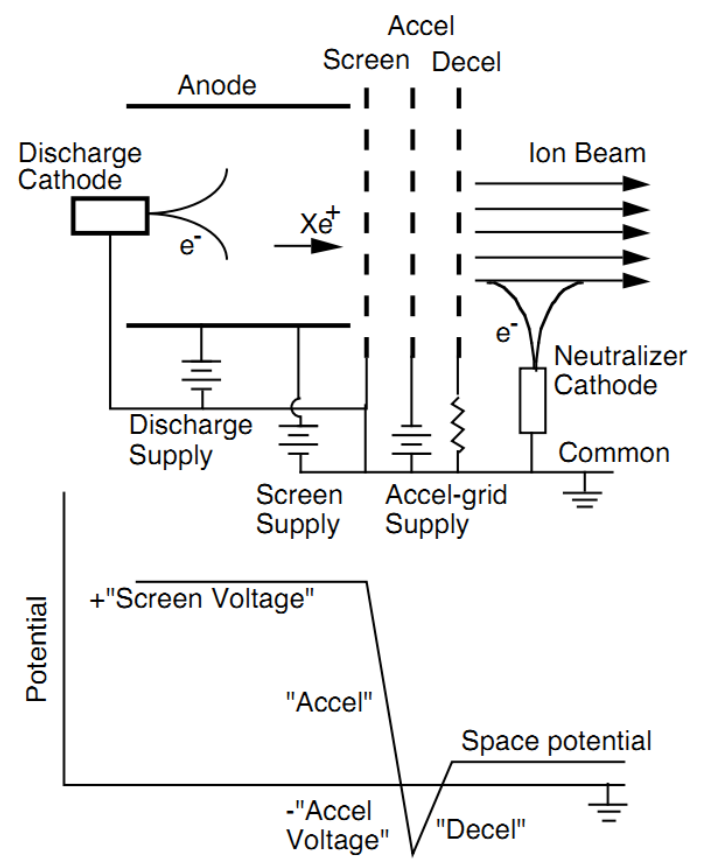

Figure 6.4: Electrical Schematic and Potential Change Diagram of a Three Grid Ion Thruster [12]

The CTFC neutralizer requires an independent power supply and feedthrough. The neutralizer cathode is powered to render the beam exiting the thruster electrically neutral to prevent ion backflow to the grids and the thruster chamber.

A comprehensive electrical schematic and component diagram is shown in Figure 6.5. 


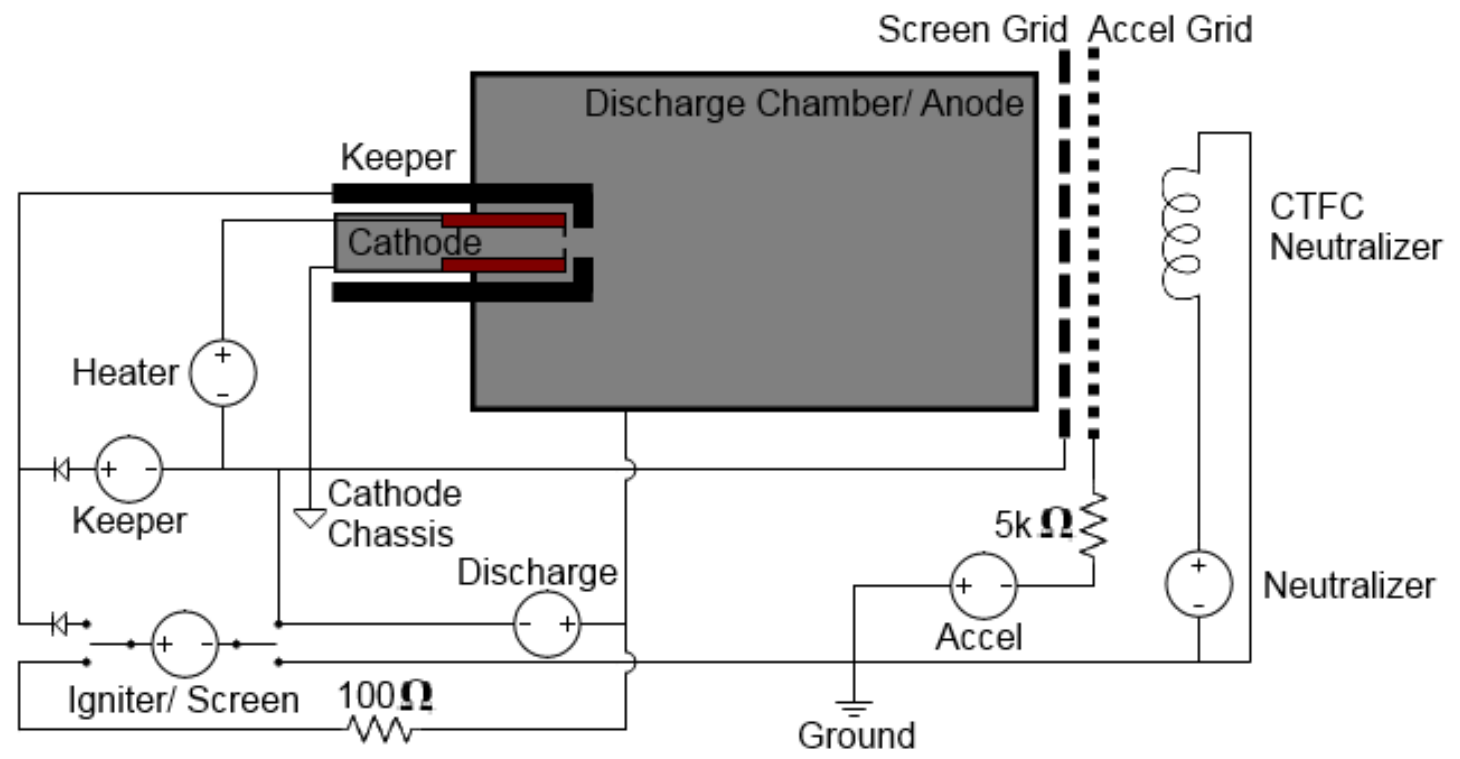

Figure 6.5: Electrical Schematic and Component Diagram of MiXI

The electrical schematic indicates a second reference ground point labeled as the Cathode Chassis. When the screen supply is connected to the discharge chamber, there is no longer earth ground to the cathode and anode and the screen supply raises the potential of the whole thruster system. To prevent shorts to ground, steps must be taken to isolate the components and the power supplies. The three power supplies that reference the cathode chassis must be installed using an isolating transformer to allow their grounds to float while still maintaining safe installation and operation. The fuel line attached to the cathode must also include an electrical isolator, discussed in the next section.

Table 6.1 is an annotated list of the power supplies and their ratings used in the operational testing of MiXI. 
Table 6.1: Power Supplies used in Operational Testing of MiXI

\begin{tabular}{|l|l|l|}
\hline Component & Power Supply & Ranges \\
\hline Cathode Heater & HP 6038A & $60 \mathrm{~V}, 10 \mathrm{~A}$ \\
\hline Cathode Keeper & Agilent N5747A & $60 \mathrm{~V}, 12.5 \mathrm{~A}$ \\
\hline Discharge/Anode & Sorensen XG 1700 300-5.6 & $300 \mathrm{~V}, 5.6 \mathrm{~A}$ \\
\hline Screen/Striker & Glassman FC1P120 & $1000 \mathrm{~V}, 0.12 \mathrm{~A}$ \\
\hline Acceleration & Glassman FC1P120 & $1000 \mathrm{~V}, 0.12 \mathrm{~A}$ \\
\hline Neutralizer CTFC & HP 6263B & $20 \mathrm{~V}, 10 \mathrm{~A}$ \\
\hline Langmuir Probe & Kepco BOP 100-1M & $\pm 100 \mathrm{~V}, \pm 1 \mathrm{~A}$ \\
\hline
\end{tabular}

\subsubsection{Propellant System Configuration}

The propellant system required for operation of the ion thruster consists of a tank of ultrahigh purity Xenon or Argon gas connected to the cathode base through 1/4" Swagelok tubing. The tubing connects through a high purity regulator, a needle valve, a digital flow meter, a diaphragm valve, a propellant feedthrough, and finally a propellant isolator before connecting to the cathode base.

The high purity regulator controls the pressure from the propellant tank to the rest of the line. The pressure on the downstream end of the regulator is maintained at a low relative pressure of about -20 psi to aid in the low flow propellant fine control. The regulator is intended to be used for high purity gases, which is necessary to prevent poisoning the cathode with any contaminants. A needle valve is used to finely control the propellant flow through the system. The flow is measured through an Omega FMA-A2300 digital flow meter. As with the pressure gauges, this meter is calibrated for $N_{2}$ gas and the appropriate conversion factors must be applied to get the true flow rate of the propellant gas. 
The diaphragm valve is used to isolate the propellant tank and equipment from the chamber when it is not under vacuum. The fuel line passes through a propellant feedthrough in the base of the vacuum chamber and through a series of tubes to a propellant isolator. The other end of the isolator is connected to the base of the cathode to complete the propellant system setup. The propellant isolator is used to keep the floating potential of the cathode base electrically isolated from ground. The propellant system setup is shown in Figure 6.6.

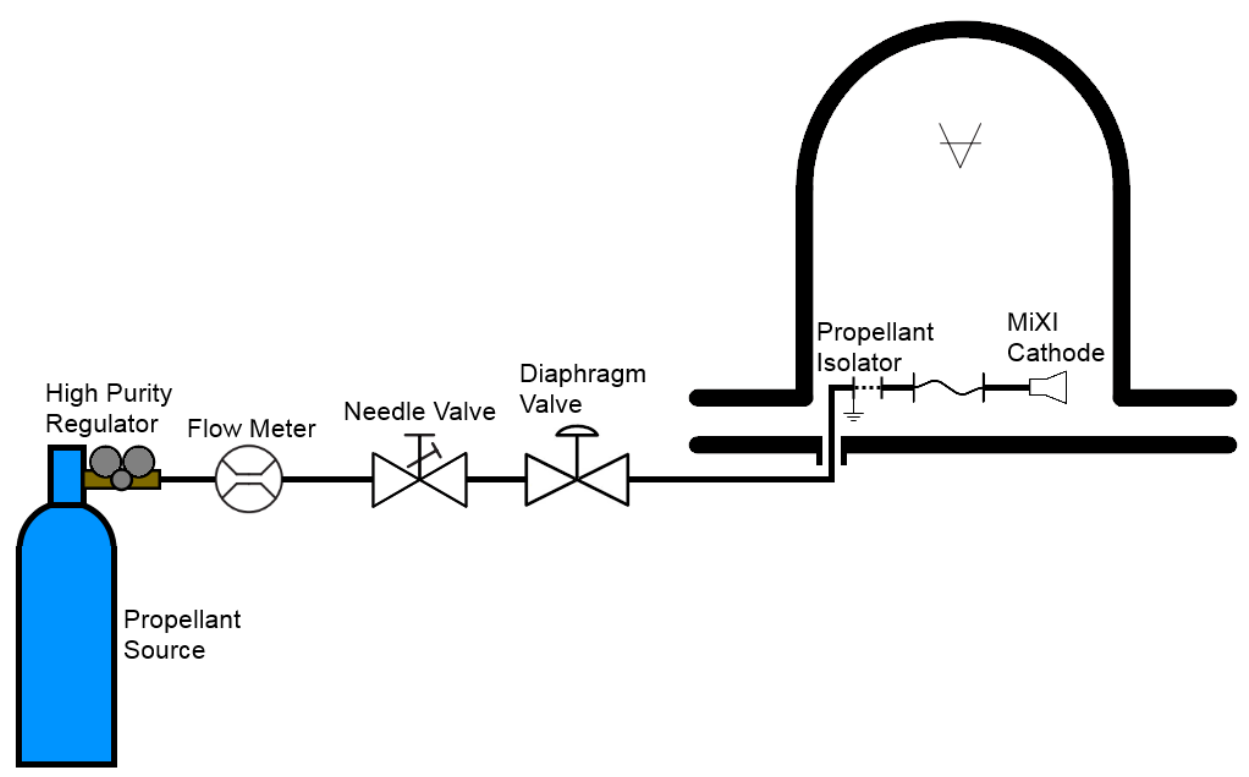

Figure 6.6: Propellant System Setup Schematic

\subsection{Testing Procedure}

Before testing could begin, steps were taken to ensure the vacuum chamber would be able to reach the desired base pressure and that the apparatus and experiment were clean. The inside of the chamber and the glass jar was cleaned and wiped down with acetone. The Orings and the sections of the chamber and lid they rested on were cleaned with Kim-wipes and water to remove any buildup on the sealing surfaces. The cryo pump was run through 
a regeneration cycle and, if necessary, the compressor was refilled with helium. Detailed information about these steps can be found in Appendix B.

The components themselves were taken apart and cleaned with Kim-wipes and acetone to remove any propellant buildup and oils from handling. After cleaning, the truster and cathode were handled with gloves to prevent contaminating the components. Testing could then proceed once the thruster and cathode were carefully constructed, properly installed, and electrically checked. Detailed information about the construction of the cathode and discharge chamber can be found in Section 2.

\subsubsection{Data Acquisition}

The main goal of testing was to determine the performance characteristics of the thruster under different operating conditions. The bulk of the data collected were readings from the various power supplies. It was also important to monitor and measure the temperature of the thruster during operation to prevent any damage to the thruster and the confinement magnets. During full testing, a single thermocouple was placed at the base of the anode at the magnet ring. Previous testing has shown that the anode base is the area that reaches the greatest temperature during operation [30]. The thermocouples required were installed in the vacuum chamber through a feedthrough and read through an Omega HH85 J,K Type Thermocouple reader.

The recordings of values such as discharge voltage and screen current had to be read directly from the power supplies. Significant effort was made to digitize the data from the power supplies through LabVIEW and National Instruments data acquisition units. All of the power supplies had some method of analog output, but due to the fact that three of the power supplies needed a floating ground, attaching the DAQs created a new ground path that made that method of data acquisition impossible. Without more advanced isolation 
equipment and data acquisition devices, the data from the power supplies needed to be recorded by hand. The sources of the data collected are shown in Table 6.2.

Table 6.2: Sources of Data Recorded from Operational Testing

\begin{tabular}{|l|c|l|}
\hline Data Source & Read & Notes \\
\hline Propellant Mass Flow Rate & SCCM & \\
\hline Heater Voltage & V & \\
\hline Heater Current & A & \\
\hline Keeper Voltage & V & \\
\hline Keeper Current & A & \\
\hline Discharge Voltage & V & \\
\hline Discharge Current & A & \\
\hline Screen Grid Voltage & V & Limited \\
\hline Screen Grid Current & $\mathrm{mA}$ & Limited \\
\hline Accel. Voltage & V & Limited \\
\hline Accel. Current & $\mathrm{mA}$ & Limited \\
\hline Anode Base Temperature & $\mathrm{deg} C$ & \\
\hline Langmuir Voltage & V & Situational \\
\hline Langmuir Current & $\mathrm{A}$ & Situational \\
\hline
\end{tabular}

The accelerator grid system was not energized at all times, therefore the data from those supplies was limited to only when ion extraction was taking place. The anode temperature was used qualitatively to determine the discharge power settings causing the greatest amount of heat deposition and to determine when it was necessary to halt testing to prevent magnet overheating. The Langmuir voltage and current was taken for only a few power settings and situations when the beam was not energized. 


\subsubsection{Hollow Cathode Stand-Alone Test}

The first set of tests involved operating the hollow cathode independently of the MiXI discharge chamber. This was done to ensure the cathode was operating correctly and would be easy to strike once the thruster was fully assembled. Previous theses indicated that delays in the ignition of the cathode could reduce operational time due to heating of the anode from the cathode heater.

The hollow cathode was electrically installed as was described previously and displayed in Figure 6.3. A discharge chamber analog was used for the initial testing. A comprehensive list of steps for the operation of the cathode can be found in Appendix C. The cathode was tested and verified that it could operate as intended before the MiXI discharge chamber was installed. Figure 6.7 shows the cathode operating in the vacuum chamber using a discharge chamber analog. In the figure, there is a distinct conical plume of plasma emanating from the cathode orifice.

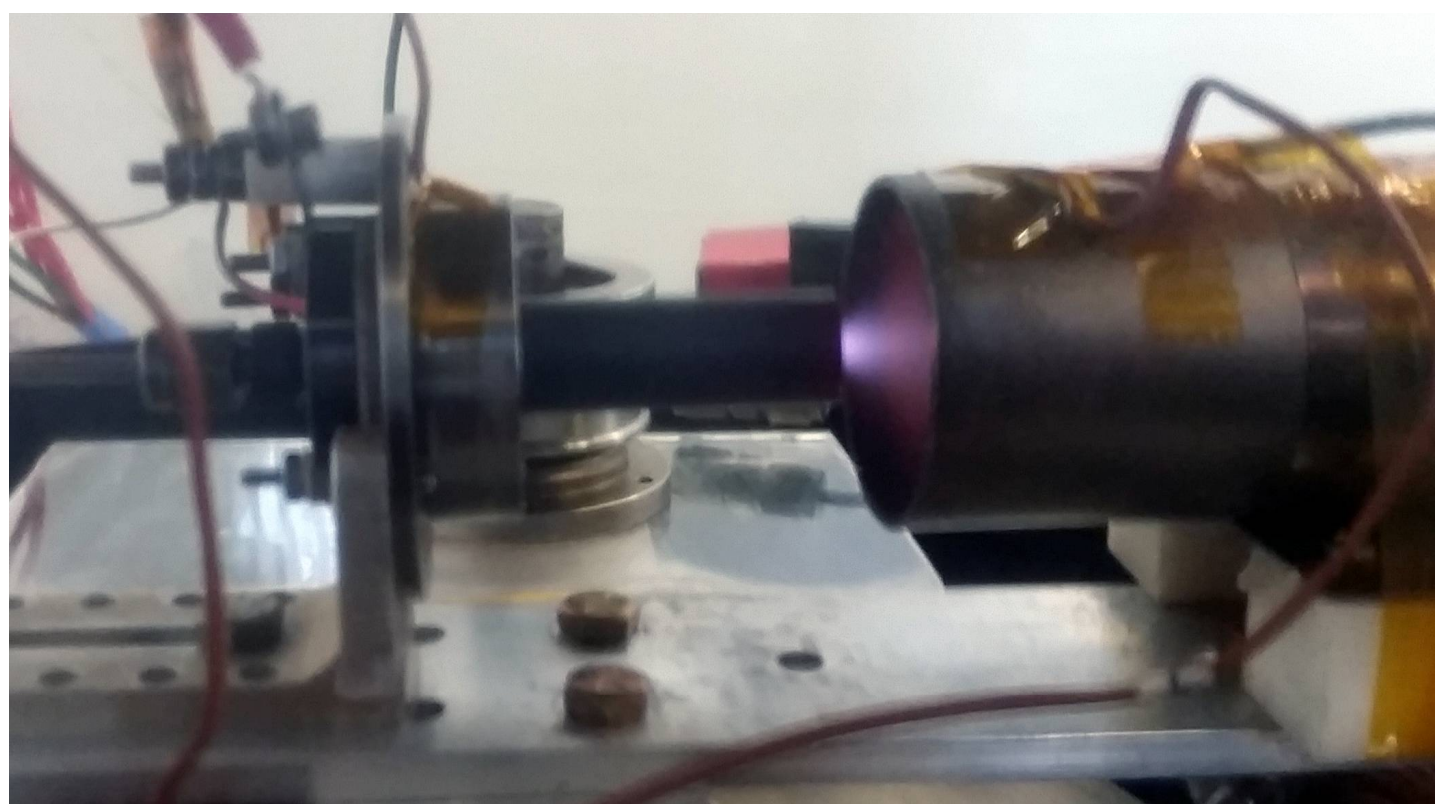

Figure 6.7: Operation of the MiXI Cathode for the Hollow Cathode Stand-Alone Tests 
Testing of the cathode alone provided some significant pieces of information important for operating the thruster as a whole. Through the stand-alone tests, the duration of time required to properly heat the insert was determined. The minimum propellant flow rate and necessary range of voltages that could strike the cathode were identified. It was also possible to reduce the heater power after the cathode had been operating while still maintaining plasma discharge; the required heater power levels and the timing of this change was also determined. The insight gained from testing the cathode alone was valuable when operating the completed thruster.

\subsubsection{MiXI Testing}

Full MiXI testing could begin after operating the cathode and verifying the electrical setup of the full system. The discharge chamber was assembled and installed using mounting hardware and placed in the vacuum chamber at the appropriate location. At the same time, a Langmuir probe was installed and fed through the vacuum chamber. The tip of the Langmuir probe was placed a few millimeters from the acceleration grid and set with the tip at the center axis of the thruster.

The discharge chamber was electrically installed as was described previously and displayed in Figure 6.5. At this point, it was critical to verify all electrical connections; mistakes in the installation could introduce problems and prevent the thruster from operating correctly. Once the electrical system was verified, testing of the MiXI thruster could begin. A comprehensive list of steps for the operation of MiXI can be found in Appendix D.

Once MiXI was lit and stable, a desired throttle point could be set and readings of the propellant flow rate, power supply settings, and thermocouple temperature were recorded. At some throttle points, the screen and acceleration grid supplies were used to apply a range of beam voltages to the thruster, or the Langmuir supply was used to gather a sweep of data from the Langmuir probe. To obtain accurate data, it was necessary to let the 
thruster thermally equalize when changing throttle points. It was also critical to monitor the temperature of the thruster at all times and halt testing if the temperature became too great. At values greater than $325 \mathrm{deg} C$, the $\mathrm{SmCo}$ magnets can become permanently damaged. To maintain magnet life, the thruster was shut down when the thermocouple read $280 \operatorname{deg} C$ at the anode base in order to provide a safe margin of temperature for the interior magnets. The thruster was required to cool between tests to allow for increased run time before magnet temperature became a concern.

The thruster was operated at throttle levels similar to previous theses $[19,30]$; the throttle ranges at which the thruster was operated is displayed in Table 6.3.

Table 6.3: Thruster Testing Throttle Levels

\begin{tabular}{|l|r|l|}
\hline Parameter & \multicolumn{1}{l|}{ Value } & Unit \\
\hline Propellant Flow Rate & $1.7-8.2$ & SCCM \\
\hline Heater Current & $0-6$ & $\mathrm{~A}$ \\
\hline Keeper Current & $0.4-2.0$ & $\mathrm{~A}$ \\
\hline Discharge Current & $0.4-2.0$ & $\mathrm{~A}$ \\
\hline Screen Grid Voltage & $100-500$ & $\mathrm{~V}$ \\
\hline Accel. Grid Voltage & $100-200$ & $\mathrm{~V}$ \\
\hline Neutralizer Current & 5 & $\mathrm{~A}$ \\
\hline Langmuir Voltage & \pm 100 & $\mathrm{~V}$ \\
\hline
\end{tabular}

At various throttle points, Langmuir probe sweeps were recorded. The Langmuir probe was used to determine the plasma characteristics of the beam and used as an approximation of the characteristics of the plasma within the discharge chamber. An interesting thing discovered was that the Langmuir probe would read large values of ion current when energizing the beam and extracting ions from the discharge chamber. This would be expected as the beam neutralization did not occur until a point past the Langmuir probe and the exit 
velocity of the ions was great enough to impact the probe even when held at high potentials. However, when the beam was engaged, using the Langmuir probe to determine the plasma parameters became impossible.

While there will be differences between the plasma recorded outside the chamber grids [22], it was decided to use the beam plasma as a direct approximation of the chamber plasma parameters. Figure 6.8 shows the installed components, including the Langmuir probe and the neutralizer CTFC, and the full thruster during operation.

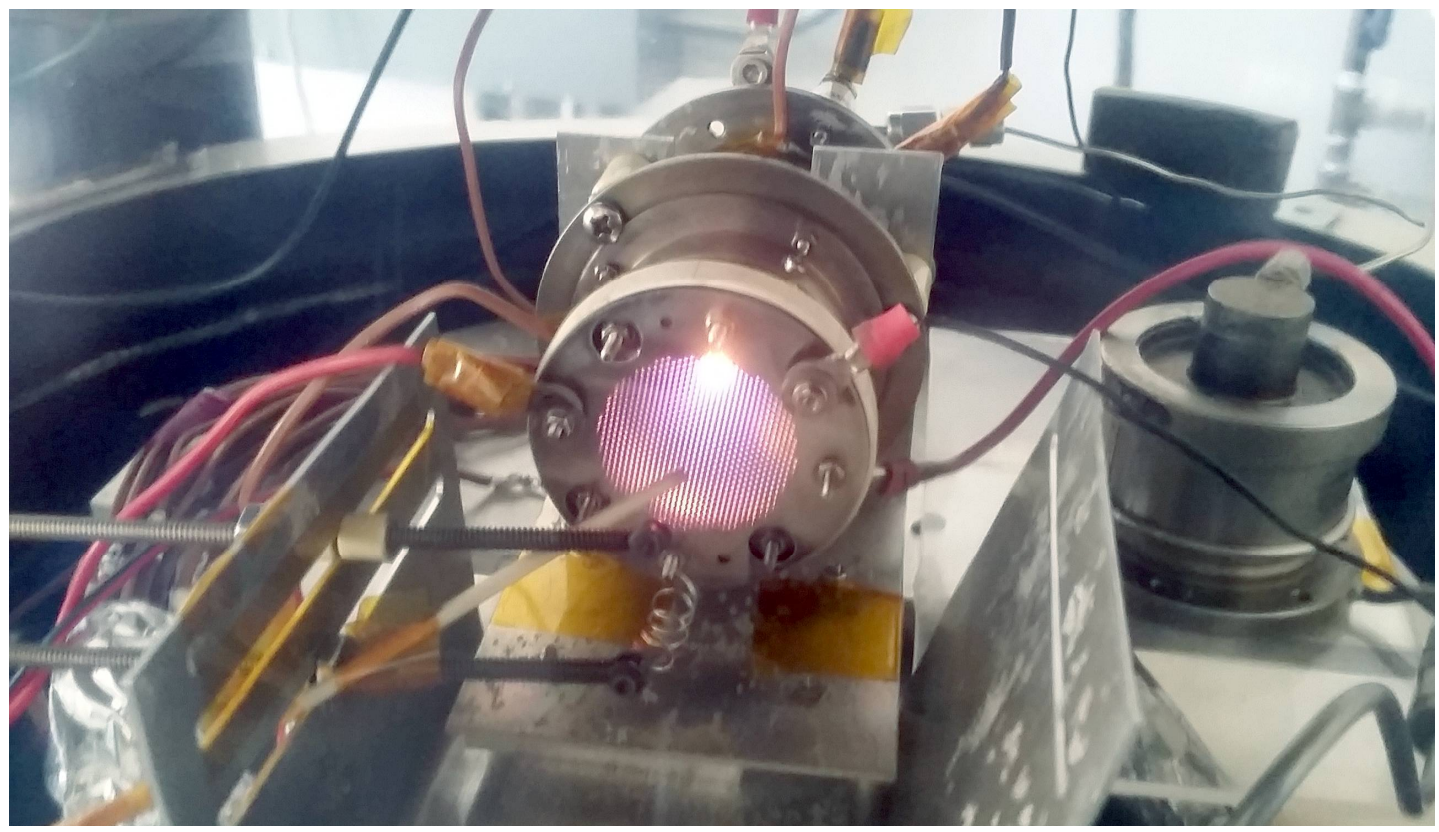

\section{Figure 6.8: Operation of the MiXI thruster. Neutralizer CTFC disabled for picture purposes}

The installed Langmuir probe is a cylindrical $0.50 \mathrm{~mm}$ diameter tungsten wire with a $1.65 \mathrm{~mm}$ lead out the end of a ceramic sheath. The probe was attached to a $\pm 100 \mathrm{~V}, 1 \mathrm{~A}$ Kepco-BOP power supply and connected in series with a $100 \Omega$ resistor. This power supply contained no digital outputs and significant effort was made to digitize the data through NI DAQs and LabVIEW, but problems with equipment and lack of specific parts meant the current data needed to be recorded by hand. Methods for Langmuir probe analysis are well documented [7], and the specifics to the analysis performed in this thesis are described in 
Appendix F. A sample trace from one of the Langmuir probe sweeps is displayed in Figure 6.9.

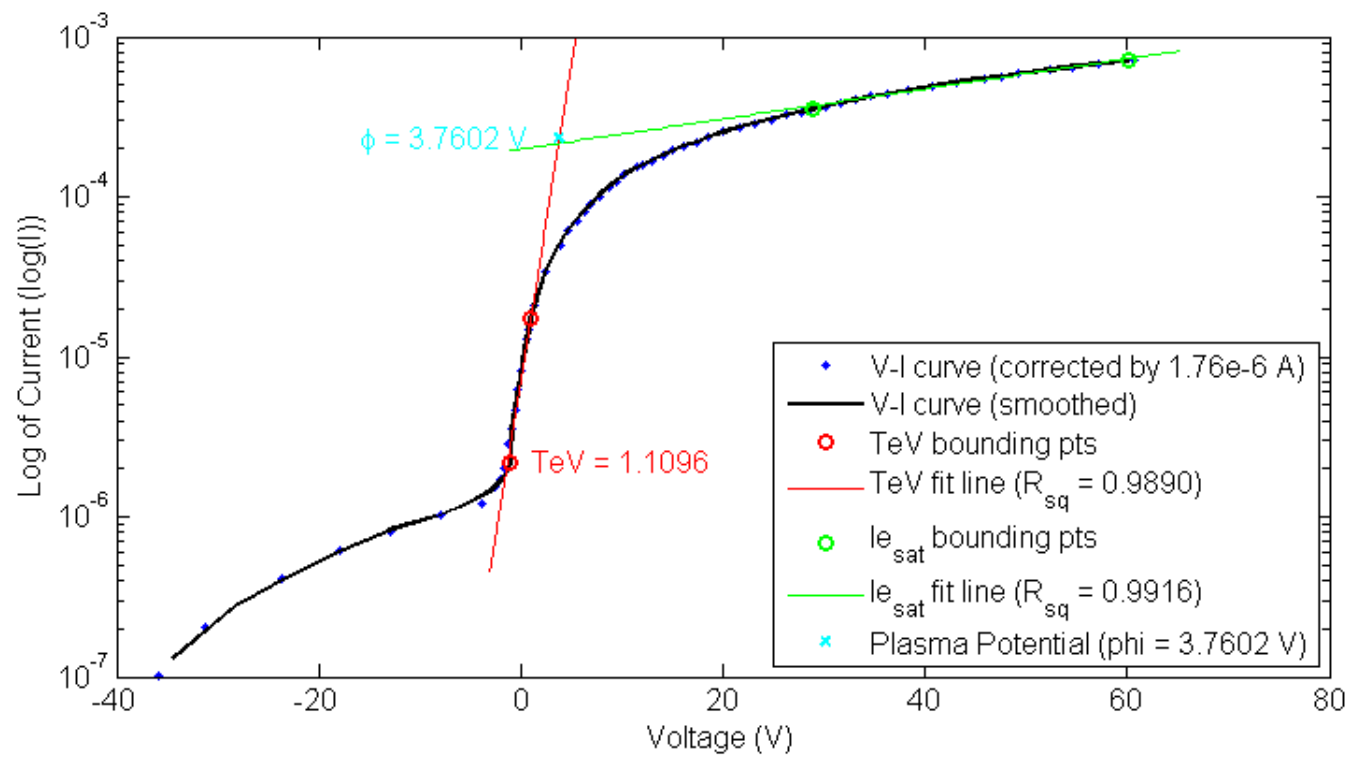

Figure 6.9: Langmuir Probe Sweep Sample, semi-log graph

Analysis of the semi-log plot of current versus voltage is the typical way to determine the plasma parameters. In this trace, the data points are corrected by the ion saturation value and bounds are placed on the linear regions to determine the electron temperature and electron saturation current value. The slope of the linear regions are used to determine the temperature of the plasma and electron saturation current and the intersections of the lines determine the plasma potential.

Operational data was recorded at a large number of throttle points, requiring a significant amount of time to complete the testing desired for this thesis. Due to cost, Argon was used for the duration of the testing instead of Xenon. This means the results from the performance model will not agree fully with testing data, however the same trends should apply to both propellants. A full analysis of the test data and the results are discussed in the following section. 


\section{Chapter 7}

\section{RESULTS}

This section presents the results of operational testing and compares the data to the theory from the performance model. The testing results were analyzed and manipulated to create performance curve analogs similar to those presented in the Model Solutions section (5). The objective of the comparison was to verify the computational model to gain confidence in it's outputs. Wile it was possible to analyze the testing data in many ways, comparing to the performance model outputs directly was not possible for this thesis. The test data was acquired using Argon gas, while the idealized models developed and used in the performance models base their calculations on Xenon gas and are not well formulated or defined for Argon gas. As was mentioned before, Argon was used because it was available in the lab and cheaper in the quantities used.

The following subsections describe the analysis of the results, numerical error analysis, and the comparisons between the performance models and the collected data.

\subsection{Data Analysis}

There were two main sets of data collected during testing: data with ion extraction and an associated beam voltage, and data from the thruster operating in discharge mode and with no ion extraction. The data with ion extraction has an extracted beam current that can be determined and used in calculations. The discharge mode of operation does not have a distinct ion beam and the thruster acts primarily as a plasma generation source. Langmuir probe data was taken of the thruster operating in discharge mode at different throttle levels to gain insight to the plasma characteristics. The methods of the analysis for the Langmuir probe are demonstrated in Appendix F. 
The test data with ion extraction was used to analyze thruster performance. In the analysis of the results, it is critical to acquire the beam characteristics from the test data. The beam voltage $V_{b}$ is the potential across which the ions are accelerated, taken as the difference between the screen and accelerator grid's potentials

$$
V_{b}=V_{s g}+\left|V_{a g}\right|
$$

where $V_{s g}$ is the set potential of the screen grid and $V_{a g}$ is the set potential of the acceleration grid. The beam voltage $I_{b}$ is determined from the measured screen grid current $I_{s g}$ amplified by the value of the screen's transparency to ions $T_{s}$, shown in the equation

$$
I_{b}=\frac{I_{s g} T_{s}}{1-T_{s}} * 1000
$$

The screen transparency value is an approximation of the screen's transparency to ions. As discussed earlier in Section 4, this value is an estimate of 0.75 . The scale value of 1000 is used to convert the milliamp reading from the power supply to amps.

With the beam characteristics determined, it is possible to calculate the discharge loss $\eta_{d}$ from the data sets with the equation

$$
\eta_{d}=\frac{V_{d} I_{d}}{I_{b}}
$$

The mass flow rate into the system is measured in SCCM and is converted to units of $\mathrm{kg} / \mathrm{s}$ by

$$
\dot{m}=\frac{\Omega_{\text {Argon }} Q_{\text {in }}}{\rho_{\text {Argon }} 60}
$$

The leading term $\Omega_{\text {Argon }}$ is a conversion factor required by the Omega flow meter to determine the flow of Argon gas instead of the calibrated N2 gas, a value of 1.44. With the beam current and the mass flow rate known, the mass utilization efficiency value $\eta_{m}$ can be calculated by the equation

$$
\eta_{m}=\frac{I_{b} M}{\dot{m} e}
$$

where $M$ is the mass of an Argon ion. 
The electrical efficiency $\eta_{e}$ is also calculated from the test data and determined by the equation

$$
\eta_{e}=\frac{V_{b} I_{b}}{V_{b} I_{b}+I_{b} \eta_{d}}
$$

The specific impulse $I_{s p}$ is determined by

$$
I_{s p}=\frac{\gamma \eta_{m}}{g_{0}} \sqrt{\frac{2 e V_{b}}{M}}
$$

where $\gamma$ is a thrust correction factor predominately dependent upon the beam flatness and the ratio of double to single ion production, discussed in sections previously. Thrust $T$ $[\mathrm{mN}]$ is calculated from the beam characteristics by the equation

$$
T=\sqrt{\frac{2 M V_{b}}{e}} I_{b} \gamma * 1000
$$

and the last performance term, the total efficiency $\eta_{T}$, is calculated by the product of the efficiency terms in the equation

$$
\eta_{T}=\gamma^{2} \eta_{m} \eta_{e}
$$

In general, the testing results showed that the performance of the tests were drastically inferior to those predicted by the performance models. This was to be expected and the results of the testing were still enlightening in many ways. It should be noted that the total power presented in the results tables does not include the power required to run the neutralizer CTFC; this neutralizer power was a constant $27.5 \mathrm{~W}$.

\subsubsection{Results: Configuration_4033s}

The first configuration analyzed was the baseline_4o33s configuration. Table 7.1 describes

a portion of the testing results for this setup, including the beam characteristics and the Langmuir probe analysis. This table showcases a progression of change of the throttle points to more easily see the direct effects they have on the results. 
Table 7.1: Testing Results, Subset 1 from Configuration_4033s

\begin{tabular}{|l|r|r|r|r|c|}
\hline Parameter & Throttle 1 & Throttle 2 & Throttle 3 & Throttle 4 & Units \\
\hline Inputs & & & & & \\
\hline Prop. Flow Rate & 2.0 & 2.0 & 2.9 & 5.8 & SCCM \\
\hline Heater Current & 2.0 & 2.0 & 2.0 & 2.0 & A \\
\hline Keeper Current & 1.0 & 1.0 & 1.0 & 1.0 & A \\
\hline Discharge Current & 2.0 & 1.0 & 1.0 & 1.5 & A \\
\hline Beam Voltage & 600 & 600 & 600 & 600 & V \\
\hline Results & & & & & \\
\hline Heater Voltage & 3.68 & 3.69 & 3.86 & 4.14 & V \\
\hline Keeper Voltage & 17.5 & 23.7 & 30.2 & 28.0 & V \\
\hline Discharge Voltage & 38.3 & 44.3 & 40.9 & 38.7 & V \\
\hline Total Power & 113.8 & 86.3 & 82.1 & 107.7 & W \\
\hline Analysis & & & & & \\
\hline Beam Current & $62.4 \pm 0.3$ & $55.8 \pm 0.3$ & $58.2 \pm 0.3$ & $68.4 \pm 0.3$ & $\mathrm{~mA}$ \\
\hline Discharge Loss & $1228 \pm 62$ & $794 \pm 80$ & $723 \pm 72$ & $849 \pm 57$ & $\mathrm{eV} /$ ion \\
\hline Mass Utilization & $0.44 \pm 0.00$ & $0.39 \pm 0.00$ & $0.29 \pm 0.00$ & $0.17 \pm 0.00$ & \\
\hline Electron Temp. & 3.23 & 4.12 & 2.03 & & $\mathrm{eV}$ \\
\hline Plasma Ion Density & $2.55 \mathrm{e} 16$ & $1.27 \mathrm{e} 16$ & $3.35 \mathrm{e} 15$ & & $n / \mathrm{m}^{3}$ \\
\hline Plasma Potential & 4.66 & 7.69 & 16.52 & - & $\mathrm{V}$ \\
\hline Electrical Eff. & $32.8 \pm 2.4$ & $43.0 \pm 7.4$ & $45.3 \pm 8.1$ & $41.4 \pm 4.7$ & $\%$ \\
\hline Specific Impulse & $2366 \pm 97$ & $2116 \pm 87$ & $1544 \pm 64$ & $908 \pm 37$ & $\mathrm{~s}$ \\
\hline Thrust & $1.36 \pm 0.06$ & $1.22 \pm 0.05$ & $1.27 \pm 0.05$ & $1.49 \pm 0.06$ & $\mathrm{mN}$ \\
\hline Total Efficiency & $13.9 \pm 1.0$ & $16.3 \pm 2.8$ & $12.5 \pm 2.2$ & $6.7 \pm 0.8$ & $\%$ \\
\hline
\end{tabular}

The four data sets presented in the table are four different throttle points of the thruster. They differ in their propellant flow rate, keeper current, and discharge current. In this series of tests, the beam voltage was held at a constant $600 \mathrm{~V}$ and the heater at a constant $2 \mathrm{~A}$. It 
was necessary to maintain the heater at this low level to prevent the electron discharge process from failing at low discharge currents.

In the first throttle setting, the keeper current was set to 1A and discharge current was set to $1 \mathrm{~A}$, resulting in a beam current of $62.4 \mathrm{~mA}$ and a total power of $113.8 \mathrm{~W}$. The discharge loss was a rather large value of $1228 \mathrm{eV} /$ ion and the mass utilization was 0.44 . From these two values, it is not surprising to see the relatively low total efficiency of $12.5 \%$.

The second throttle point had the same $1 \mathrm{~A}$ on the keeper while having a discharge current. The keeper and discharge voltages are slightly higher in this case, and the beam current is slightly lower. Both the discharge loss and mass utilization values are decreased, leading to a smaller specific impulse and thrust but having a greater total efficiency while using less power. Reducing the discharge current is shown in this case to drastically lower the discharge loss and create a similarly performing thruster with higher efficiency.

The third throttle setting is similar to Throttle 2 but with an increased mass flow rate. The keeper voltage is slightly increased while the discharge voltage is reduced, leading to a smaller total power of $82.1 \mathrm{~W}$. The beam current is raised slightly and the discharge loss is decreased slightly. This throttle setting has a lower mass utilization value and a lower specific impulse, yet similar levels of thrust, when compared to the first two throttle settings. Overall, increasing the flow rate led to a thruster with reduced efficiency.

To further exemplify the performance impacts when changing the propellant flow rate, the fourth throttle point was run with a $1.5 \mathrm{~A}$ discharge current and doubled the propellant flow rate. The potentials of the keeper and discharge are similar to that of Throttle 3 , resulting in a larger total power. The beam current in this case is increased, but the discharge loss is increased and the mass utilization value is decreased. This leads to a thruster that has very low specific impulse and total efficiency compared to the other throttle levels.

The Langmuir probe data was acquired for three throttle points and the outputs are also displayed in the testing results table. The electron temperature determined is consistent 
with discharge chamber plasma from other ion thrusters [22]. The ion density predicted is similar to the primary electron density output by the performance model, though test results were determined with different propellants, causing discrepancy. The plasma potentials determined are also a higher value that expected, indicating a large potential drop across the plasma sheath and possible over-confinement of the bulk plasma.

Table 7.2 displayed for this configuration includes tests run without any additional heater current. Many cathodes are able to sustain operation without the continued use of the heater as they become self heating once the thruster is in operation. This table also showcases a progression of throttle points to facilitate comparison. 
Table 7.2: Testing Results, Subset 2 from Configuration_4033s

\begin{tabular}{|l|r|r|r|r|c|}
\hline Parameter & Throttle 5 & Throttle 6 & Throttle 7 & Throttle 8 & Units \\
\hline Inputs & & & & & \\
\hline Prop. Flow Rate & 2.9 & 2.9 & 2.9 & 1.7 & SCCM \\
\hline Heater Current & 2.0 & 2.0 & 0.0 & 0.0 & A \\
\hline Keeper Current & 2.0 & 2.0 & 2.0 & 2.0 & A \\
\hline Discharge Current & 2.0 & 0.4 & 0.4 & 0.4 & A \\
\hline Beam Voltage & 600 & 600 & 600 & 600 & V \\
\hline Results & & & & & \\
\hline Heater Voltage & 4.44 & 3.60 & 0.00 & 0.00 & V \\
\hline Keeper Voltage & 31.6 & 31.5 & 35.7 & 38.8 & V \\
\hline Discharge Voltage & 33.4 & 27.0 & 27.9 & 31.0 & V \\
\hline Total Power & 160.0 & 101.9 & 91.8 & 103.0 & W \\
\hline Analysis & & & & & \\
\hline Beam Current & $160.5 \pm 0.3$ & $101.1 \pm 0.3$ & $47.3 \pm 0.3$ & $66.6 \pm 0.3$ & $\mathrm{~mA}$ \\
\hline Discharge Loss & $416 \pm 21$ & $115 \pm 27$ & $236 \pm 59$ & $186 \pm 47$ & $\mathrm{eV} / \mathrm{ion}$ \\
\hline Mass Utilization & $0.79 \pm 0.00$ & $0.50 \pm 0.00$ & $0.23 \pm 0.00$ & $0.55 \pm 0.00$ & \\
\hline Electron Temp. & 1.11 & 3.23 & - & - & $\mathrm{eV}$ \\
\hline Plasma Ion Density & $3.97 \mathrm{e} 15$ & $1.22 \mathrm{e} 16$ & - & - & $n / m^{3}$ \\
\hline Plasma Potential & 3.76 & 11.34 & - & - & $\mathrm{V}$ \\
\hline Electrical Eff. & $49.0 \pm 4.6$ & $83.9 \pm 67.7$ & $71.8 \pm 53.5$ & $76.3 \pm 61.9$ & $\%$ \\
\hline Specific Impulse & $3478 \pm 174$ & $2683 \pm 110$ & $1254 \pm 52$ & $2946 \pm 121$ & $\mathrm{~s}$ \\
\hline Thrust & $2.86 \pm 0.14$ & $2.21 \pm 0.09$ & $1.03 \pm 0.04$ & $1.45 \pm 0.06$ & $\mathrm{mN}$ \\
\hline Total Efficiency & $37.3 \pm 3.5$ & $40.2 \pm 32.4$ & $16.1 \pm 12.0$ & $40.1 \pm 32.5$ & $\%$ \\
\hline
\end{tabular}

The throttle points in this table are different than those presented in Table 7.1 in that the keeper current is held at a constant $2 \mathrm{~A}$ instead of $1 \mathrm{~A}$. The fifth throttle point also has a $2 \mathrm{~A}$ discharge current, resulting in a large amount of power required at $160 \mathrm{~W}$. However, the beam current is a large $160.5 \mathrm{~mA}$, higher than anything presented prior, and the dis- 
charge loss is only $416 \mathrm{eV} /$ ion. With a mass utilization value of 0.79 , this throttle point is quite efficient at $37.3 \%$ compared to all previously analyzed. This throttle point also has a large specific impulse and thrust value. The increase in the keeper current shows a marked improvement to the performance of the thruster at the cost of increased power required overall.

The sixth throttle point reduces the discharge current to further explore the trend discussed previously. In this test, the discharge current was reduced from $2 \mathrm{~A}$ to $0.4 \mathrm{~A}$; the resultant total power is reduced as is the beam current. The discharge loss is drastically lowered to $115 \mathrm{eV} /$ ion, similar to what was shown between throttle points in the first results subset. While the mass utilization value was decreases compared to Throttle 5, the electrical efficiency is greatly increased resulting in a higher total efficiency value of $04.2 \%$.

The seventh throttle point is assessed at the same keeper and discharge currents, but with no added heater power. At this setting, the keeper voltage increases while the discharge voltage stays almost constant resulting in a slightly decreased total power from Throttle 6. For Throttle 7, the beam current and the mass utilization is more than halved while the discharge loss is doubled. The while the electrical efficiency is a large value, the resultant total efficiency is drastically reduced with the heater power removed.

The eighth throttle point is an interesting result in that, with the slight decrease in the propellant flow rate, the performance increased drastically to near the level of Throttle 6 . The test points in Table 7.1 showed increasing the flow rate 0.9 SCCM decreased the total efficiency by about $3.5 \%$. The result in Throttle 8 shows a disproportional increase of $24 \%$ when decreasing the propellant flow rate by $0.9 \mathrm{SCCM}$ when compared to the results in Table 7.1.

To more concisely display the performance characteristics of the thruster configuration, it was prudent to generate charts evaluating the discharge loss as a function of the mass utilization efficiency, generating performance curves as discussed in the Computa- 
tional Model Solutions section (5). Figure 7.1 is a representation of a performance curve generated from the testing data. The error markers in the figure contain one standard deviation of error in the data, assuming perfect knowledge of the screen transparency. Details on the error analysis is contained in the Numerical Error Analysis subsection (7.1.5). In this case, the displayed data is only for data points with a discharge voltage between $25-30 \mathrm{~V}$; the different beam currents are classified by colors and identified in the legend.

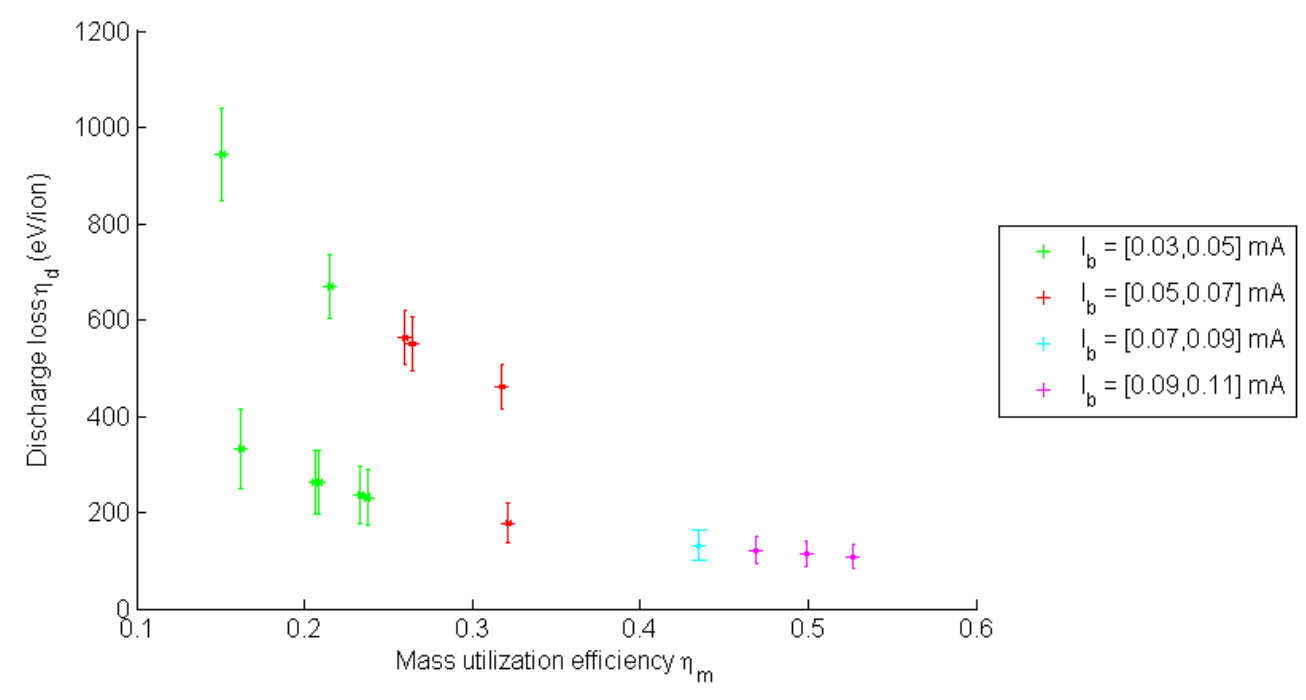

Figure 7.1: Data Analysis Performance Curve; Baseline Configuration, Discharge Voltage 25-30V

It is immediately apparent that the data presented demonstrates inferior performance when compared to the idealized performance curves generated from the model or demonstrated in other classes of thrusters. The discharge loss is generally greater and the mass utilization efficiency much less than the generated performance curves or those from the NSTAR thruster. While demonstrating poorer performance, the data displayed in the figure still suggests some trends that have manifested in the test results.

Overall, it is shown that the mass utilization is increased as the beam current is increased. The cluster of green markers on the left of the chart contain the lowest beam current and the mass utilization values. The higher mass utilization values are associated 
with the higher beam currents. Also, as the mass utilization value is increased, the discharge loss is shown to decrease. This is opposite the usual trend, however, these mass utilization values are significantly far from the asymptotic boundary where the discharge loss must increase.

Figure 7.2 is identical in concept to Figure 7.1, but with a discharge voltage limit between 35-40V.

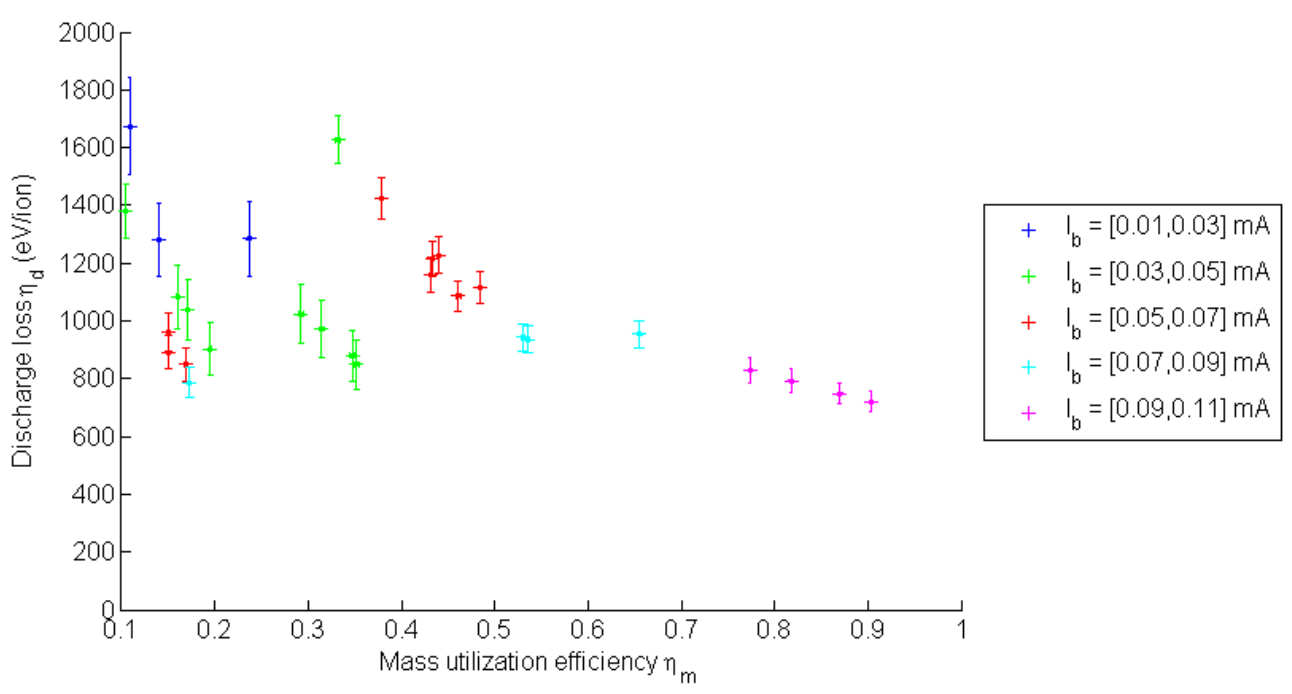

Figure 7.2: Data Analysis Performance Curve; Baseline Configuration, Discharge Voltage 35-40V

The first thing to note about this figure is the average range of discharge loss values the data set occupies. Compared to the previous discharge voltage range's average discharge loss of $344 \mathrm{eV} /$ ion, the average discharge loss for this voltage range is $1053 \mathrm{eV} /$ ion. While there are some non-conforming data points, the same general trend discovered in the previous figure holds true: the larger mass utilization values are associated with the larger beam currents and the discharge loss decreases with increasing mass utilization. 


\subsubsection{Results: Configuration_4033t}

Another configuration was built and tested in a similar manner to the baseline configuration.

Table 7.3 describes a portion of the testing results for this setup.

Table 7.3: Testing Results, Subset 1 from Configuration_4o33t

\begin{tabular}{|c|c|c|c|c|c|}
\hline Parameter & Throttle 1 & Throttle 2 & Throttle 3 & Throttle 4 & Units \\
\hline \multicolumn{6}{|l|}{ Inputs } \\
\hline Prop. Flow Rate & 3.2 & 3.2 & 2.9 & 6.6 & SCCM \\
\hline Heater Current & 2.0 & 2.0 & 2.0 & 2.0 & A \\
\hline Keeper Current & 1.50 & 1.50 & 1.50 & 1.00 & A \\
\hline Discharge Current & 2.00 & 2.00 & 1.50 & 2.00 & A \\
\hline Beam Voltage & 600 & 600 & 600 & 600 & $\mathrm{~V}$ \\
\hline \multicolumn{6}{|l|}{ Results } \\
\hline Heater Voltage & 3.82 & 3.80 & 3.71 & 3.64 & $\mathrm{~V}$ \\
\hline Keeper Voltage & 29.8 & 28.9 & 26.4 & 24.6 & $\mathrm{~V}$ \\
\hline Discharge Voltage & 27.3 & 28.3 & 25.2 & 26.3 & $\mathrm{~V}$ \\
\hline Total Power & 128.0 & 128.3 & 90.8 & 102.8 & $\mathrm{~W}$ \\
\hline \multicolumn{6}{|l|}{ Analysis } \\
\hline Beam Current & $106.2 \pm 0.3$ & $105.6 \pm 0.3$ & $85.5 \pm 0.3$ & $92.7 \pm 0.3$ & $\mathrm{~mA}$ \\
\hline Discharge Loss & $514 \pm 26$ & $536 \pm 27$ & $437 \pm 29$ & $567 \pm 29$ & $\mathrm{eV} /$ ion \\
\hline Mass Utilization & $0.48 \pm 0.00$ & $0.47 \pm 0.00$ & $0.42 \pm 0.00$ & $0.20 \pm 0.00$ & \\
\hline Electron Temp. & 4.13 & 4.77 & - & - & $\mathrm{eV}$ \\
\hline Plasma Ion Density & $1.24 \mathrm{e} 16$ & $1.50 \mathrm{e} 16$ & - & - & $n / m^{3}$ \\
\hline Plasma Potential & 33.5 & 30.5 & - & - & $\mathrm{V}$ \\
\hline Electrical Eff. & $53.9 \pm 5.6$ & $52.8 \pm 5.4$ & $57.9 \pm 8.7$ & $51.4 \pm 5.1$ & $\%$ \\
\hline Specific Impulse & $2562 \pm 105$ & $2548 \pm 104$ & $2269 \pm 93$ & $1070 \pm 44$ & $\mathrm{~s}$ \\
\hline Thrust & $2.32 \pm 0.09$ & $2.31 \pm 0.09$ & $1.87 \pm 0.08$ & $2.02 \pm 0.08$ & $\mathrm{mN}$ \\
\hline Total Efficiency & $24.6 \pm 2.6$ & $24.0 \pm 2.5$ & $23.4 \pm 3.5$ & $9.8 \pm 1.0$ & $\%$ \\
\hline
\end{tabular}


Throttle points 1 and 2 have the same throttle and the values collected were from two separate tests. The data between the two shows remarkable consistency and provides a measure of confidence in the precision of the data. The two throttle points were run with $1.5 \mathrm{~A}$ on the keeper and $2 \mathrm{~A}$ on the discharge chamber. This ultimately lead to a total power of about $128 \mathrm{~W}$ and a beam current of about 106mA. The discharge loss was an average of $524 \mathrm{eV} /$ ion and the total efficiency was an average of $24.4 \%$.

Due to time constraints and temperature concerns when operating the thruster, it was not always possible to take consistent Langmuir probe data at all throttle points. The Langmuir probe data from the similar throttle points indicate an electron temperature of about $4.4 \mathrm{eV}$, larger than what the performance model predicts, but this is a value consistent with other ion thruster discharge chamber plasma and results from the previous configuration.

The third throttle point decreases both the propellant flow rate and the discharge current. From testing of the baseline configuration, the beam current and the discharge loss should decrease with these changes to the throttle point. This result is shown to be consistent with results in the previous configuration in that regard. The resulting total efficiency of this throttle point is $23.4 \%$, slightly less than that of Throttles 1 and 2 .

The fourth data point is interesting in that it is run with a much larger propellant mass flow rate than the previously discussed throttle levels. While the keeper and discharge values are different, there is significant change in the mass utilization value and the total efficiency value which can be attributed to the large increase in the propellant flow rate, consistent with results from Table 7.1. While a larger amount of fuel is required to start the thruster, the more fuel consistently pumped into the discharge chamber during operation is shown to decrease performance and significantly negatively impact the mass utilization efficiency. The detrimental effects of increasing the propellant flow rate would suggest that decreasing the rate may improve performance of the thruster, however it was difficult to 
maintain the very low levels of flow and maintain plasma production within the discharge chamber to fully explore this.

Table 7.4 displays a subset of data collected from this configuration when the thruster was operated with no added heater power.

Table 7.4: Testing Results, Subset 2 from Configuration_4o33t

\begin{tabular}{|c|c|c|c|c|c|c|}
\hline Parameter & Throt. 5 & Throt. 6 & Throt. 7 & Throt. 8 & Throt. 9 & Units \\
\hline \multicolumn{7}{|l|}{ Inputs } \\
\hline Prop. Flow Rate & 1.7 & 2.9 & 2.9 & 2.9 & 2.9 & SCCM \\
\hline Heater Current & 0.0 & 0.0 & 0.0 & 0.0 & 0.0 & A \\
\hline Keeper Current & 2.0 & 2.0 & 2.0 & 2.0 & 1.0 & A \\
\hline Discharge Current & 0.4 & 0.4 & 1.0 & 2.0 & 0.4 & A \\
\hline Beam Voltage & 600 & 600 & 600 & 600 & 600 & V \\
\hline \multicolumn{7}{|l|}{ Results } \\
\hline Heater Voltage & 0.00 & 0.00 & 0.00 & 0.00 & 0.00 & V \\
\hline Keeper Voltage & 37.3 & 33.6 & 25.9 & 21.3 & 34.3 & V \\
\hline Discharge Voltage & 21.2 & 19.8 & 20.9 & 20.1 & 25.2 & $\mathrm{~V}$ \\
\hline Total Power & 97.5 & 92.5 & 83.1 & 96.0 & 53.0 & $\mathrm{~W}$ \\
\hline \multicolumn{7}{|l|}{ Analysis } \\
\hline Beam Current & $74.7 \pm 0.3$ & $88.2 \pm 0.3$ & $53.1 \pm 0.3$ & $67.5 \pm 0.3$ & $44.1 \pm 0.3$ & $\mathrm{~mA}$ \\
\hline Discharge Loss & $114 \pm 28$ & $90 \pm 22$ & $394 \pm 39$ & $596 \pm 30$ & $229 \pm 57$ & $\mathrm{eV} /$ ion \\
\hline Mass Utilization & $0.61 \pm 0.00$ & $0.44 \pm 0.00$ & $0.26 \pm 0.00$ & $0.33 \pm 0.00$ & $0.22 \pm 0.00$ & \\
\hline Electron Temp. & - & - & - & - & - & $\mathrm{eV}$ \\
\hline Plasma Ion Density & - & - & - & - & - & $n / m^{3}$ \\
\hline Plasma Potential & - & - & - & - & - & $\mathrm{V}$ \\
\hline Electrical Eff. & $84.1 \pm 72.8$ & $87.0 \pm 72.6$ & $60.4 \pm 14.3$ & $50.2 \pm 4.9$ & $72.4 \pm 54.7$ & $\%$ \\
\hline Specific Impulse & $3304 \pm 136$ & $2341 \pm 96$ & $1409 \pm 58$ & $1719 \pm 74$ & $1170 \pm 48$ & $\mathrm{~s}$ \\
\hline Thrust & $1.63 \pm 0.07$ & $1.93 \pm 0.08$ & $1.16 \pm 0.05$ & $1.47 \pm 0.06$ & $0.96 \pm 0.04$ & $\mathrm{mN}$ \\
\hline Total Efficiency & $49.6 \pm 43.0$ & $36.4 \pm 30.3$ & $15.2 \pm 3.6$ & $16.1 \pm 1.6$ & $15.1 \pm 11.4$ & $\%$ \\
\hline
\end{tabular}


The fifth throttle point is identical to the eighth throttle point for the baseline configuration, shown in Table 7.2. Both operate with a 2.0 SCCM propellant flow rate, 2A keeper current, and 0.4A discharge current. The analysis shows the discharge loss is very small and the mass utilization value quite large, resulting in a very efficient thruster throttle level. In this configuration, the discharge loss is $114 \mathrm{eV} /$ ion and the total efficiency is $49.6 \%$.

The sixth throttle point increases the propellant flow rate by 0.9 SCCM from Throttle 5 while maintaining the same keeper and discharge currents. According to previous testing, it would be expected that the beam current and discharge loss would increase while the mass utilization would decrease; the testing with this configuration shows the beam current does increase and the mass utilization decreases, but the discharge loss decreased with the increased propellant flow rate. The lower mass utilization value ultimately leads to a lower total efficiency, a result consistent with the baseline configuration testing.

The seventh and eighth throttle points increase the discharge current incrementally while keeping the propellant flow rate and the keeper current constant. From baseline configuration testing, it would be expected that the discharge loss would increase and the total efficiency would decrease with the increasing discharge current. The results table shows that the discharge loss does incrementally increase with the increasing discharge current; the mass utilization and total efficiency are also reduced for both throttle points.

The last throttle point decreases the keeper current when compared to Throttle 6. Previous testing would suggest the mass utilization value will drastically decrease resulting in a lower thrust and less efficient thruster. This trend holds consistent with this configuration with the mass utilization being reduced to 0.22 , half that of when the keeper current was at $2 \mathrm{~A}$, and the total efficiency is reduced to $15.1 \%$.

Figure 7.3 displays performance charts similar to those displayed for the baseline configuration. In this case, the discharge voltage is bounded between $25-30 \mathrm{~V}$. 


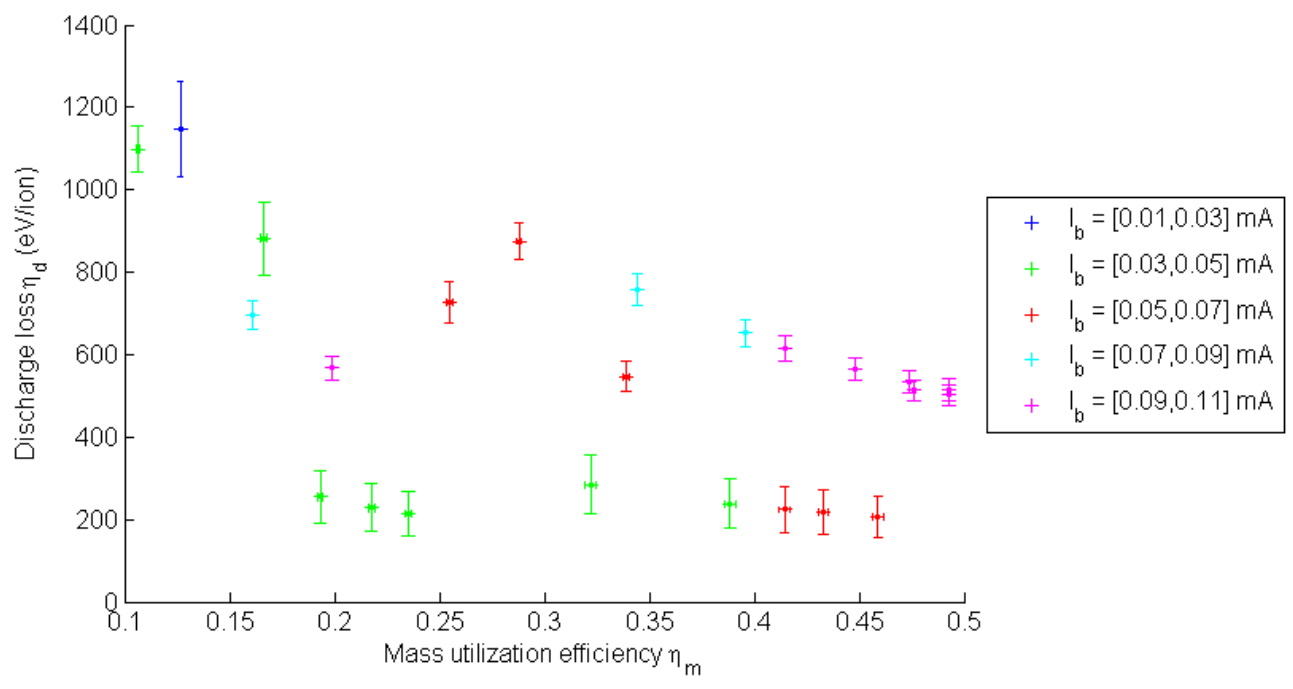

Figure 7.3: Data Analysis Performance Curve; _4o33t Configuration, Discharge Voltage $25-30 \mathrm{~V}$

The data from this configuration is much less congruent compared to the baseline configuration data. In general, it can still be shown that the larger mass utilization values are produced by the larger beam current values, though there are numerous groupings of points that do not perfectly follow this trend. It should also be noted that every point within these discharge voltage bounds exhibit a mass utilization value less than 0.5.

Figure 7.4 displays performance charts with the discharge voltage bounded between $35-40 \mathrm{~V}$. 


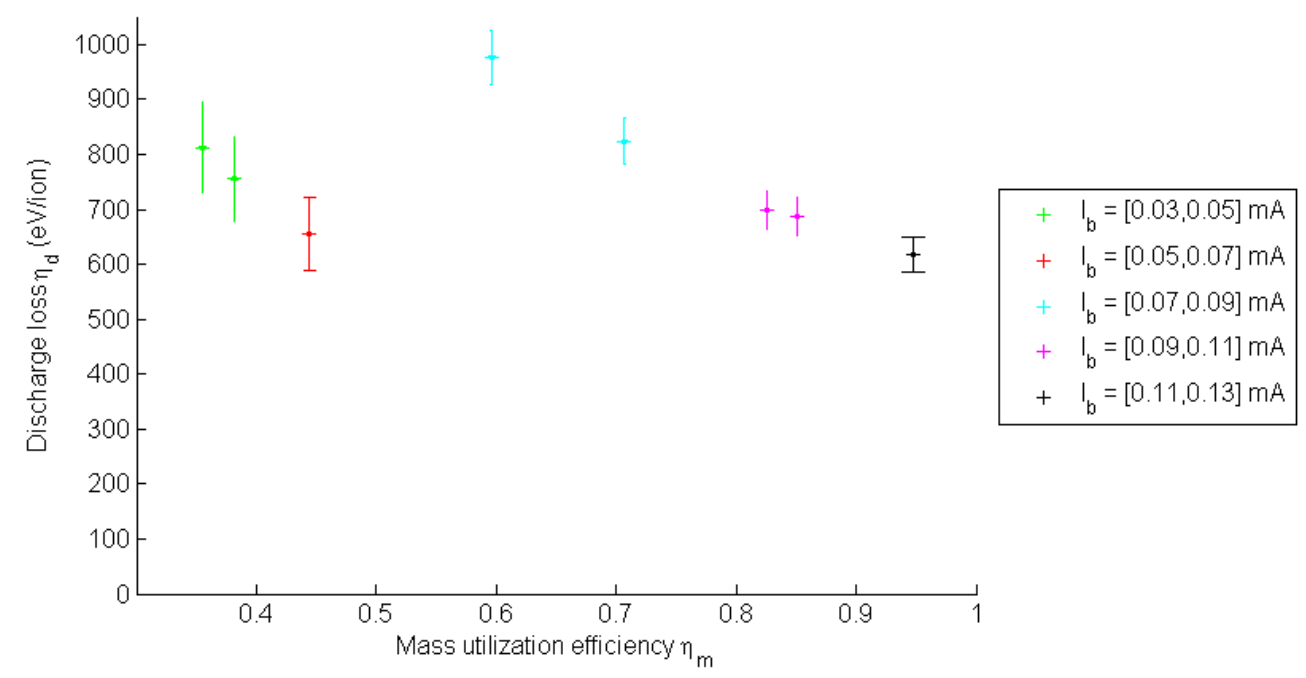

Figure 7.4: Data Analysis Performance Curve;_4o33t Configuration, Discharge Voltage 35-40V

In this figure, the trend of increasing mass utilization with increasing beam current is followed exactly. The largest mass utilization values coincide with the largest beam current values. It should also be noted that the discharge loss values occur at larger mass utilization values for this range of discharge voltages. This set of data is different from the set displayed for the baseline configuration with the same discharge voltage bounds. In this configuration, the average discharge loss is $753 \mathrm{eV} /$ ion, much lower and more similar to the 25-30V discharge voltage data set.

\subsubsection{Results: Configuration_4031s}

The third configuration analyzed involved installing the anode cusp spacer ring and was the configuration_4031s. Table 7.5 describes a portion of the testing results for this setup, including the beam characteristics and the Langmuir probe analysis. 
Table 7.5: Testing Results from Configuration_4031s

\begin{tabular}{|c|c|c|c|c|c|}
\hline Parameter & Throttle 1 & Throttle 2 & Throttle 3 & Throttle 4 & Units \\
\hline \multicolumn{6}{|l|}{ Inputs } \\
\hline Prop. Flow Rate & 2.9 & 2.9 & 5.8 & 2.9 & SCCM \\
\hline Heater Current & 2.0 & 3.0 & 2.0 & 3.0 & A \\
\hline Keeper Current & 1.0 & 1.5 & 2.0 & 0.4 & A \\
\hline Discharge Current & 1.0 & 2.0 & 2.0 & 0.4 & A \\
\hline Beam Voltage & 600 & 600 & 600 & 600 & $\mathrm{~V}$ \\
\hline \multicolumn{6}{|l|}{ Results } \\
\hline Heater Voltage & 4.10 & 5.90 & 4.16 & 5.82 & $\mathrm{~V}$ \\
\hline Keeper Voltage & 32.6 & 24.5 & 25.0 & 33.8 & $\mathrm{~V}$ \\
\hline Discharge Voltage & 35.3 & 29.6 & 27.3 & 37.2 & $\mathrm{~V}$ \\
\hline Total Power & 91.4 & 118.8 & 129.7 & 53.0 & $\mathrm{~W}$ \\
\hline \multicolumn{6}{|l|}{ Analysis } \\
\hline Beam Current & $60.6 \pm 0.3$ & $89.7 \pm 0.3$ & $85.2 \pm 0.3$ & $37.5 \pm 0.3$ & $\mathrm{~mA}$ \\
\hline Discharge Loss & $604 \pm 60$ & $651 \pm 33$ & $641 \pm 32$ & $397 \pm 99$ & $\mathrm{eV} /$ ion \\
\hline Mass Utilization & $0.30 \pm 0.00$ & $0.44 \pm 0.00$ & $0.21 \pm 0.00$ & $0.19 \pm 0.00$ & \\
\hline Electron Temp. & 5.68 & 1.27 & 3.24 & 4.76 & $\mathrm{eV}$ \\
\hline Plasma Ion Density & $2.73 \mathrm{e} 15$ & $6.34 \mathrm{e} 15$ & $4.64 \mathrm{e} 15$ & $4.74 \mathrm{e} 15$ & $n / m^{3}$ \\
\hline Plasma Potential & 17.96 & 3.53 & 10.32 & 14.58 & $\mathrm{~V}$ \\
\hline Electrical Eff. & $49.8 \pm 9.7$ & $48.0 \pm 4.5$ & $48.4 \pm 4.6$ & $60.2 \pm 35.5$ & $\%$ \\
\hline Specific Impulse & $1608 \pm 66$ & $2381 \pm 9.8$ & $1131 \pm 46$ & $995 \pm 41$ & $\mathrm{~s}$ \\
\hline Thrust & $1.32 \pm 0.05$ & $1.96 \pm 0.08$ & $1.86 \pm 0.08$ & $0.82 \pm 0.03$ & $\mathrm{mN}$ \\
\hline Total Efficiency & $14.3 \pm 2.8$ & $20.4 \pm 1.9$ & $9.8 \pm 0.9$ & $10.7 \pm 6.3$ & $\%$ \\
\hline
\end{tabular}

This set of tests show reasonable agreement with tests in other configurations in terms of efficiency, electron temperature, and beam parameters. The throttle levels show variation to the operation and efficiency of the thruster based on their input propellant flow rate and the component currents. The first throttle setting utilizes 1A on both the keeper and 
discharge, resulting in a beam current of $60.6 \mathrm{~mA}$ and a total power of $91.4 \mathrm{~W}$. The discharge loss demonstrates inefficiencies which are apparent in the electrical and total efficiency values, resulting in a total efficiency of $14.3 \%$.

The second throttle level increases the heater, keeper, and discharge current. The heater current was increased in this case to see the effect the added heat would have on the insert and electron generation out of the cathode. While the beam current is substantially larger, this effect is primarily from the increase in the discharge current. There is also a marked improvement to the mass utilization and the total efficiency when compared to the first throttle point.

The third throttle point was another test with increased propellant mass flow rate. As with the previous test increasing the flow rate, the keeper and discharge voltages are not drastically different, there is a significant change the mass utilization value and the total efficiency value, resulting in much less efficient thruster operation overall.

The fourth throttle point reduced the keeper and discharge currents to explore the possibility of operating the thruster with very low total power levels. The keeper and discharge voltages raised in response to the lowered current values, yet the resulting total power value was significantly lower than all other tests displayed here. The beam current and the discharge loss were smaller, yet the mass utilization efficiency was drastically different, similar to levels when the propellant flow rate was increased. While the electrical efficiency was greater than the other tests, the specific impulse, thrust, and total efficiency were all lacking. It was required to have the $3 \mathrm{~A}$ heater value for this throttle point to maintain plasma production from the cathode. 


\subsubsection{Results: Configuration_2o31s}

The last configuration constructed and tested was the _2o31s configuration. Table 7.6 describes a portion of the testing results for this setup, including the beam characteristics and the Langmuir probe analysis.

Table 7.6: Testing Results from Configuration_2o31s

\begin{tabular}{|c|c|c|c|c|c|}
\hline Parameter & Throttle 1 & Throttle 2 & Throttle 3 & Throttle 4 & Units \\
\hline \multicolumn{6}{|l|}{ Inputs } \\
\hline Prop. Flow Rate & 2.9 & 2.9 & 2.9 & 2.9 & SCCM \\
\hline Heater Current & 2.0 & 2.0 & 0.0 & 2.0 & A \\
\hline Keeper Current & 1.0 & 1.0 & 1.0 & 2.0 & A \\
\hline Discharge Current & 2.0 & 1.5 & 0.8 & 0.4 & $\mathrm{~A}$ \\
\hline Beam Voltage & 600 & 600 & 600 & 600 & $\mathrm{~V}$ \\
\hline \multicolumn{6}{|l|}{ Results } \\
\hline Heater Voltage & 3.86 & 3.83 & 0.00 & 3.69 & $\mathrm{~V}$ \\
\hline Keeper Voltage & 22.1 & 27.3 & 27.6 & 25.0 & $\mathrm{~V}$ \\
\hline Discharge Voltage & 28.5 & 31.2 & 32.8 & 28.2 & $\mathrm{~V}$ \\
\hline Total Power & 105.6 & 98.4 & 60.1 & 69.1 & $\mathrm{~W}$ \\
\hline \multicolumn{6}{|l|}{ Analysis } \\
\hline Beam Current & $94.5 \pm 0.3$ & $83.7 \pm 0.3$ & $37.5 \pm 0.3$ & - & $\mathrm{mA}$ \\
\hline Discharge Loss & $603 \pm 30$ & $559 \pm 37$ & $683 \pm 86$ & - & eV/ion \\
\hline Mass Utilization & $0.47 \pm 0.00$ & $0.41 \pm 0.00$ & $0.19 \pm 0.00$ & - & \\
\hline Electron Temp. & 2.63 & 3.95 & 5.62 & 2.88 & $\mathrm{eV}$ \\
\hline Plasma Ion Density & $1.12 \mathrm{e} 16$ & $2.28 \mathrm{e} 15$ & $2.12 \mathrm{e} 15$ & $4.03 \mathrm{e} 15$ & $n / m^{3}$ \\
\hline Plasma Potential & 7.28 & 18.87 & 15.10 & 8.64 & $\mathrm{~V}$ \\
\hline Electrical Eff. & $49.9 \pm 4.9$ & $51.8 \pm 6.9$ & $46.8 \pm 10.8$ & - & $\%$ \\
\hline Specific Impulse & $2508 \pm 103$ & $2221 \pm 91$ & $995 \pm 41$ & - & $\mathrm{s}$ \\
\hline Thrust & $2.06 \pm 0.08$ & $1.83 \pm 0.07$ & $0.82 \pm 0.03$ & - & $\mathrm{mN}$ \\
\hline Total Efficiency & $22.3 \pm 2.2$ & $20.5 \pm 2.8$ & $8.3 \pm 1.9$ & - & $\%$ \\
\hline
\end{tabular}


The first throttle point is similar to tests presented for other configurations in terms of inputs, results, and analysis. The total power consumed was $105.6 \mathrm{~W}$ and there was a beam current of $94.5 \mathrm{~mA}$. The discharge loss was a similar value of $603 \mathrm{eV} /$ ion and the total efficiency was $22.3 \%$.

The second throttle point lowered the discharge current from Throttle 1 to a value of 1.5A. Previous configuration analysis would suggest the beam current and discharge loss will decrease with this change; this prediction is consistent with the results in this table between Throttle 1 and 2. While the discharge loss and the electrical efficiency decreased, the mass utilization also decreased and this throttle setting was less efficient compared to Throttle 1.

The third throttle point was tested without any additional heater power. In this test, the beam current was also reduced leading to a much lower total power required. The lower beam current and increased discharge loss led to a much lower mass utilization value and total efficiency. The specific impulse and the thrust also suffered due to the change in heater settings. The poor performance from this and other configuration tests suggests that the increased energy directly into the cathode insert assists plasma production and propellant utilization, resulting in increased performance.

The fourth throttle point was a test with the thruster operating in discharge mode, without ion extraction from the plasma. This throttle point was explored to determine operation with low discharge power levels and no additional heater power. While it was possible to acquire a partial Langmuir probe data set to determine the electron temperature, the discharge was unstable and it was not possible to obtain a reliable set of beam voltage values to analyze and compare. 


\subsubsection{Numerical Error Analysis}

A study on the numerical error was performed to determine the standard deviation of the analysis and results. The error analysis is based on the concept of the propagation of uncertainty in error applied to measurements and calculations [36]. Generally, the accuracy of the results were limited by the precision of the power supplies and the propellant flow meter, however, constant fluctuations in the discharge and keeper power supplies limited the precision actually attainable from the power supplies. Many of the analysis equations are also based on the value of the screen grid's transparency to ions $T_{s}$. This was a critical piece of information required for the analysis that was assumed, introducing an immense amount of error. Two runs of error analysis were performed, one assuming the transparency value was perfect and one including an estimated error in the transparency value.

Table 7.7 presents the setup values for the numerical error analysis case assuming perfect knowledge of the screen transparency value.

Table 7.7: Numerical Error Analysis Setup Values

\begin{tabular}{|c|c|c|c|c|c|}
\hline $\begin{array}{l}d Q_{i n} \\
(\mathrm{SCCM})\end{array}$ & $\begin{array}{l}d V_{d}, d V_{k} \\
(\mathrm{~V})\end{array}$ & $\begin{array}{l}d I_{d}, d I_{k} \\
(\mathrm{~A})\end{array}$ & $\begin{array}{l}d V_{s g}, d V_{a g} \\
\text { (V) }\end{array}$ & $\begin{array}{l}d I_{s g}, d I_{a g} \\
\text { (A) }\end{array}$ & $d T_{s}$ \\
\hline 0.02 & 0.01 & 0.1 & 1 & 0.1 & 0 \\
\hline
\end{tabular}

Table 7.8 presents a sample of the results from the numerical error analysis. This table assumes the error in the screen transparency is zero. Shown in this table are the average magnitudes of the error and the percent error. 
Table 7.8: Numerical Error Analysis Results, Assuming Perfect $T_{S}$

\begin{tabular}{|l|r|r|r|r|r|r|r|}
\hline Parameter & \multicolumn{1}{l|}{\begin{tabular}{l}
\multicolumn{1}{l|}{$\begin{array}{l}I_{b}(\mathrm{eV} / \\
(\mathrm{mA})\end{array}$} \\
ion)
\end{tabular}} & \multicolumn{1}{l|}{$\eta_{m}$} & $\eta_{e}(\%)$ & $I_{s p}(\mathrm{~s})$ & $\mathrm{T}(\mathrm{mN})$ & $\eta_{T}(\%)$ \\
\hline Error Value & 0.3 & 46 & $<0.01$ & 12.8 & 70 & 0.06 & 3.9 \\
\hline Error Percent & 0.5 & 10.0 & 0.5 & 21.2 & 3.8 & 3.9 & 21.2 \\
\hline
\end{tabular}

From the table, it is easy to see that the error values and percentages are large for some parameters dependent upon the knowledge of the discharge and keeper voltages. There are some parameters with error less than 5\%, including the mass utilization, the beam current, the mass utilization, the specific impulse, and the thrust. The electrical and total efficiency have an error value of $21.2 \%$, a significantly large value which suggests many of the results displayed and analysis of the results may be within the limits of the error.

Table 7.9 displays the results from the error analysis when the screen transparency value is assumed to not be perfect. The setup is identical as shown in Table 7.7 except the value for $d T_{s}$ is now 0.02 .

Table 7.9: Numerical Error Analysis Results, Assuming Perfect $T_{S}$

\begin{tabular}{|l|r|r|r|r|r|r|r|}
\hline Parameter & \multicolumn{1}{l|}{\begin{tabular}{l}
\multicolumn{1}{l|}{$\begin{array}{l}\eta_{b}(\mathrm{eV} / \\
(\mathrm{mA})\end{array}$} \\
ion)
\end{tabular}} & \multicolumn{1}{l|}{$\eta_{m}$} & $\eta_{e}(\%)$ & $I_{s p}(\mathrm{~s})$ & $\mathrm{T}(\mathrm{mN})$ & $\eta_{T}(\%)$ \\
\hline Error Value & 27.2 & 200 & 0.13 & 55.3 & 782 & 0.64 & 18.6 \\
\hline Error Percent & 42.2 & 43.4 & 42.2 & 91.8 & 42.4 & 42.4 & 101.0 \\
\hline
\end{tabular}

It is immediately apparent that there is a large amount of error that arises from the estimation of the screen transparency value. With even a small amount of error in this value, the calculation for the beam current becomes skewed and all of the resulting values deviate. The results presented in this thesis assume a perfect value of the screen transparency, otherwise no conclusions from the data would have any bearing. Through the error analysis, 
it has been shown that there is manageable error if the screen transparency value is well defined, but the error dominates the results if the transparency is not well defined.

\subsection{Configuration and Performance Comparison}

It was necessary to directly compare the test results to highlight the performance differences between the configurations. Additionally, while the performance model and test results were based on different propellants, an effort was made to create analogous performance curves from the test data to compare to the performance model outputs.

The most in depth and extensive testing was completed for the baseline configuration _4o33s and the second configuration _4o33t. These configurations were also tested with added heater power and without, when possible. Incongruent testing data meant not all points were able to be exactly compared between the first two configurations and configurations_4o31s and_2o31s.

Table 7.10 presents the comparisons between the baseline configuration and data points for configurations_4o31s and_2o31s. 
Table 7.10: Testing Results Comparison Between Configurations

\begin{tabular}{|c|c|c|c|c|c|}
\hline Parameter & $\begin{array}{l}\text { Baseline } \\
\text { Throttle } 1\end{array}$ & $\begin{array}{l}\text { _4o31s } \\
\text { Throttle } 1\end{array}$ & $\begin{array}{l}\text { Baseline } \\
\text { Throttle } 2\end{array}$ & $\begin{array}{l}\text { _2o31s } \\
\text { Throttle } 2\end{array}$ & Units \\
\hline \multicolumn{6}{|l|}{ Inputs } \\
\hline Prop. Flow Rate & 2.9 & 2.9 & 2.0 & 2.9 & SCCM \\
\hline Heater Current & 2.0 & 2.0 & 2.0 & 2.0 & A \\
\hline Keeper Current & 1.0 & 1.0 & 1.0 & 1.0 & A \\
\hline Discharge Current & 1.0 & 1.0 & 2.0 & 2.0 & A \\
\hline Beam Voltage & 600 & 600 & 600 & 600 & $\mathrm{~V}$ \\
\hline \multicolumn{6}{|l|}{ Results } \\
\hline Heater Voltage & 3.68 & 4.10 & 3.68 & 3.86 & V \\
\hline Keeper Voltage & 30.2 & 32.6 & 17.5 & 22.1 & $\mathrm{~V}$ \\
\hline Discharge Voltage & 40.9 & 35.3 & 38.3 & 28.5 & $\mathrm{~V}$ \\
\hline Total Power & 82.1 & 91.4 & 113.8 & 105.6 & $\mathrm{~W}$ \\
\hline \multicolumn{6}{|l|}{ Analysis } \\
\hline Beam Current & $58.2 \pm 0.3$ & $60.6 \pm 0.3$ & $62.4 \pm 0.3$ & $94.5 \pm 0.3$ & $\mathrm{~mA}$ \\
\hline Discharge Loss & $723 \pm 72$ & $604 \pm 60$ & $1228 \pm 62$ & $603 \pm 30$ & $\mathrm{eV} /$ ion \\
\hline Mass Utilization & $0.29 \pm 0.00$ & $0.30 \pm 0.00$ & $0.44 \pm 0.00$ & $0.47 \pm 0.00$ & \\
\hline Electron Temp. & 2.03 & 5.68 & 3.23 & 2.63 & $\mathrm{eV}$ \\
\hline Plasma Ion Density & $3.35 \mathrm{e} 15$ & $2.73 \mathrm{e} 15$ & $2.55 \mathrm{e} 16$ & $1.12 \mathrm{e} 16$ & $n / m^{3}$ \\
\hline Plasma Potential & 16.52 & 17.96 & 4.66 & 7.28 & $\mathrm{~V}$ \\
\hline Electrical Eff. & $45.3 \pm 8.1$ & $49.8 \pm 9.7$ & $32.8 \pm 2.4$ & $49.9 \pm 4.9$ & $\%$ \\
\hline Specific Impulse & $1544 \pm 64$ & $1608 \pm 66$ & $2366 \pm 97$ & $2508 \pm 103$ & S \\
\hline Thrust & $1.27 \pm 0.05$ & $1.32 \pm 0.05$ & $1.36 \pm 0.06$ & $2.06 \pm 0.08$ & $\mathrm{mN}$ \\
\hline Total Efficiency & $12.5 \pm 2.2$ & $14.3 \pm 2.8$ & $13.9 \pm 1.0$ & $22.3 \pm 2.2$ & $\%$ \\
\hline
\end{tabular}

The first throttle point is a direct comparison between two configurations. There are slight differences in the discharge and keeper voltages and resultant total power, however the beam voltages and mass utilization values are very similar. It is also interesting to note 
the similarities between the plasma parameters and the specific impulse and thrust values, indicating very similar operation between the two configurations. Ultimately, the electrical and resultant total efficiency is greater for the _4o31s configuration, indicating the baseline configuration is inferior to the _4o31s configuration.

The second throttle point is a similar comparison between the baseline and _2o31s configurations. There are differences between the keeper and discharge voltages and resultant total power, while the plasma parameters are similar between the cases. At this throttle point, the beam current, discharge loss, and electrical efficiency are drastically different between the configurations; this results in the _2o31s configuration outperforming the baseline configuration by a significant margin.

When running the optimization solutions on the computational models, the optimally designed_4o31s configuration thruster had a maximum total efficiency $4.19 \%$ less than the baseline configuration. The same analysis showed the baseline configuration outperforming the _2o31s configuration by $5.81 \%$. The results from the optimization are not necessarily applicable to identical throttle settings as the optimization does not necessarily occur at the same throttle point, but one might expect the same trend to hold across all throttle points.

There were many more points with identical throttle inputs to compare the difference between the baseline configuration and configuration _4o33t. Figure 7.5 represents the array of discharge voltages versus mass utilization for the common throttle settings between the two configurations. The different markers distinguish the configurations while the colors differentiate the beam currents. 


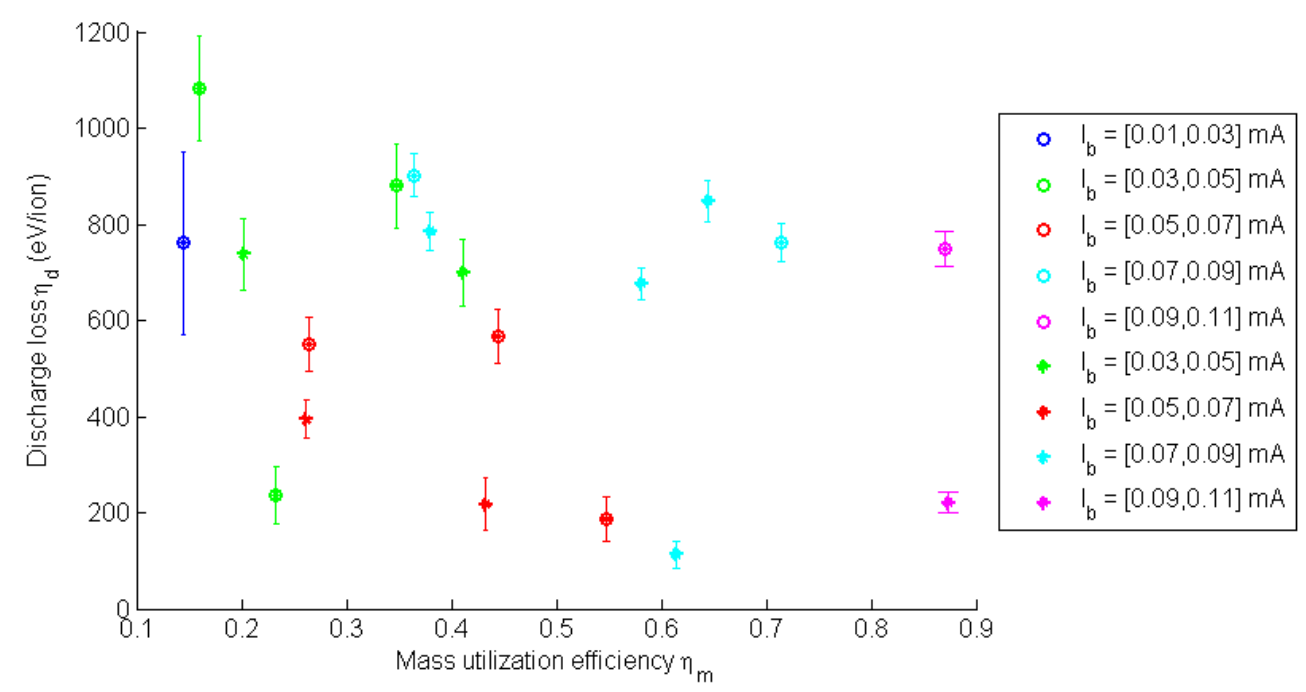

Figure 7.5: Performance Model Comparison between Baseline and_4o33t Configurations. Beam Voltage 600V, Discharge Voltage Unconstrained (Legend notes: Circle $=$ Baseline, Diamond $=\_4033 t$ )

The points contained within this data set were run with either 1.7 or 2.9 SCCM propellant flow rate and with a beam voltage of $600 \mathrm{~V}$. This entire data set consists of throttle points run without any added heater power. The results from the throttle points in this chart were not categorized by discharge voltage, having between $20-40 \mathrm{~V}$ on the anode.

From the figure, there are no immediately apparent trends distinguishing the two configurations. Further analysis reveals the average discharge loss values of the baseline configuration is $668 \mathrm{eV} /$ ion and the average mass utilization is 0.41 . The average discharge loss for the _4o33t configuration is $559 \mathrm{eV} /$ ion and the average mass utilization is 0.44 . A direct comparison between a wide range of coincident throttle points reveals that the _4o33t configuration outperformed the baseline configuration on average.

Though analysis of the performance and of the graphs presented may indicate improved operation between throttle points or configurations, it is important to realize there is a significant amount of error present in the data. Conclusions based on the data are not 
completely reliable must be reevaluated with better measuring equipment and data logging before any true conclusions can be made.

\subsubsection{Performance Model Comparison}

Performance curves were generated that encompassed the range of throttle points encountered during testing. Ideally, the test data and performance curves would coincide for identical throttle points, however with the large amount of error present, it was expected that there would be inconsistencies. Figure 7.6 depicts five performance model outputs at varying beam currents and the data set contained within the discharge voltages $30-35 \mathrm{~V}$.

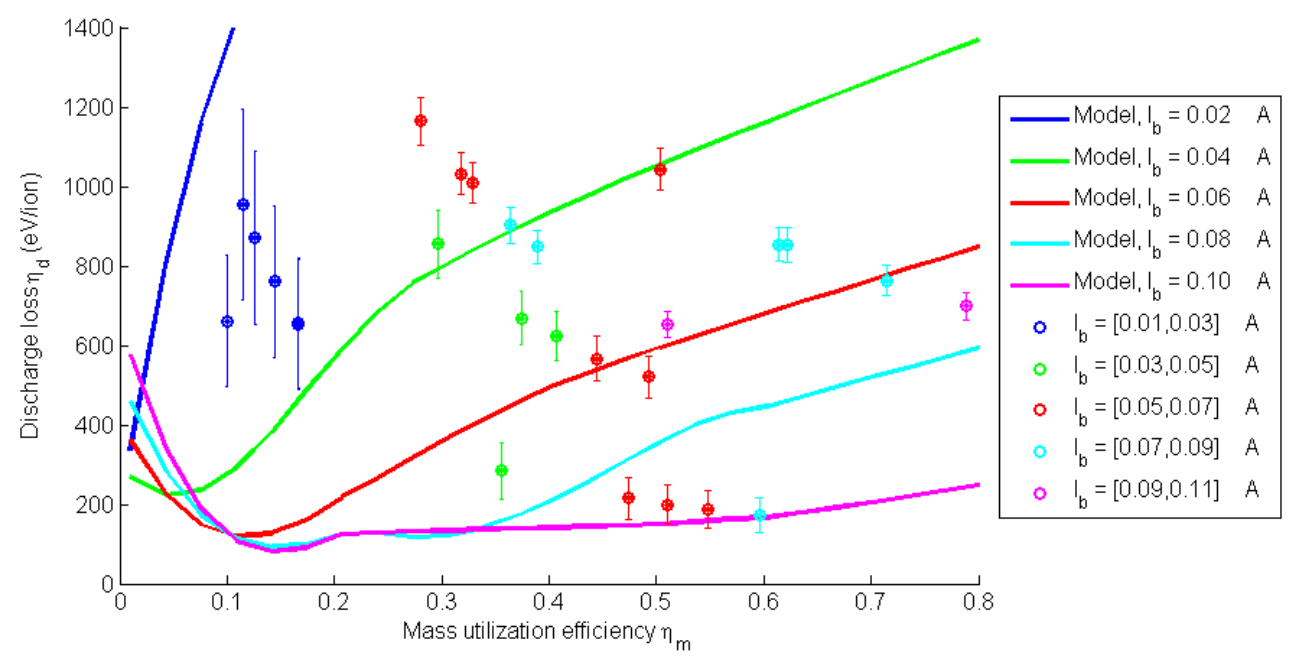

Figure 7.6: Testing Results and Performance Model Comparison; Baseline Configuration, Discharge Voltage 30-35V

From the figure, it is apparent that the model and the data do not align perfectly and there are very few points that agree between the data and the performance model. The models demonstrate there should be decreasing discharge loss and increasing mass utilization with increasing beam current. At very low values of beam current and mass utilization efficiency, the model breaks down and the outputs are flawed. This is because there is not enough energy to excite and ionize the neutrals and maintain a stable plasma discharge. 
While the data collected does not match perfectly to the model traces, clusters within the data sets follow the trends shown by the models. Considering a condition with a constant discharge loss, increasing the mass utilization efficiency requires increasing the beam current to maintain that constant discharge loss. Clusters of the test data follow this trend; for a relatively constant discharge loss, increasing the mass utilization correlates to an increasing beam current, though not across the same spans or the same levels. While the numbers between the data and the model are not consistent, the trends are consistent between.

The performance model also outputs expected values of electron temperature and plasma potential. The two tables shown of the optimized model outputs, Tables 5.4 and 5.7, predicts electron temperatures between 0.77 and $2.30 \mathrm{eV}$, with numerous cases exceeding these values. The Langmuir probe results show that the electron temperature of the plasma outside of the discharge chamber are values between 1.11 and $5.68 \mathrm{eV}$, indicating agreement between the performance model outputs and the test data.

\subsection{MiXI Version Comparison}

The results obtained from testing MiXI-CP-V3 varied from the performance demonstrated by operational testing from previous theses. In this thesis, the thruster was operated at a wide range of throttle settings, varying the propellant flow rate, keeper current, discharge current, and beam voltage. Characterizing the performance of the thruster as a single value was not representative of the operational range of MiXI-CP-V3.

Table 7.11 compares the span of performance parameters demonstrated by this set of testing. The performance parameters are compared to those presented by the initial testing performed by Knapp [19], where applicable. It should be noted that the testing performed by Knapp utilized Xenon gas as the propellant; it would be expected that the performance demonstrated using Argon would be inferior to those with Xenon at identical throttle levels. 
Table 7.11: MiXI-CP-V3 Performance Parameters Comparison

\begin{tabular}{|l|c|c|c|}
\hline Parameter & Knapp Testing & Baseline Testing & Units \\
\hline Inputs & & & \\
\hline Propellant & Xenon & Argon & \\
\hline Prop. Flow Rate & $0.55-1.06$ & $1.70-4.00$ & SCCM \\
\hline Keeper Current & - & $0.4-2.0$ & A \\
\hline Discharge Current & $0.5-1.5$ & $0.4-2.0$ & A \\
\hline Beam Voltage & $700-850$ & $200-700$ & V \\
\hline Results & - & & \\
\hline Keeper Voltage & - & $22.4-44.6$ & V \\
\hline Discharge Voltage & $90-150$ & $51-160$ & W \\
\hline Total Power & & & \\
\hline Analysis & $26.0-41.0$ & $14.0-160.5$ & $\mathrm{~mA}$ \\
\hline Beam Current & $500-1000$ & $109-1673$ & $\mathrm{eV} / \mathrm{ion}$ \\
\hline Discharge Loss & $0.30-0.77$ & $0.10-0.90$ & \\
\hline Mass Utilization & $18.0-28.0$ & $11.0-86.6$ & $\%$ \\
\hline Electrical Efficiency & $1500-3300$ & $310-5246$ & $\mathrm{~s}$ \\
\hline Specific Impulse & $1.20-2.00$ & $0.18-2.86$ & $\mathrm{mN}$ \\
\hline Thrust & - & $1.3-45.9$ & $\%$ \\
\hline Total Efficiency & & & \\
\hline
\end{tabular}

The testing results presented in this thesis was performed at a higher propellant mass flow rate than the previous thesis, but at similar discharge currents and beam voltages. The resulting parameters from testing performed in this thesis entirely encompasses the data presented previously. With the wide range of beam voltages, there were numerous points 
demonstrating poor mass utilization performance and with a low beam current, resulting in a low total efficiency. There were also numerous points exceeding the previous data, even while using Argon as the propellant. 


\section{Chapter 8}

\section{CONCLUSION}

A computational performance model was created and used to asses the operation of the MiXI-CP-V3 thruster. This computational model was able to asses the performance of the thruster at the baseline configuration and numerous other possible magnetic configurations. Analysis of the performance model indicated that there are numerous ways to increase the performance and efficiency of the thruster. It was shown that changing the beam current, discharge voltage, and expected mass utilization will effect the discharge loss and the total efficiency of the thruster. The analysis of the performance curves reveals optimal throttle settings for the MiXI thruster. The performance model was able to asses the theoretical operation of the thruster over a wide range of inputs.

In an effort to increase the theoretical performance of the thruster, numerous magnetic field configurations were created and analyzed. The configurations varied in their cusp locations and number of magnets in the cusps, resulting in drastically different cusp strengths and internal magnetic field geometry. The magnetic field strength at the cusps and the closed loop field strength are used in the computational performance model and changing the inputs by using a different magnetic field configuration resulted in a different performance at identical throttle points. A total of forty-eight magnetic configurations were created and analyzed within the performance model.

The performance model was used within an optimizer to determine idealized throttle settings that yielded the greatest total efficiency. The optimizer was capable of varying the throttle inputs and utilizing the different magnetic field configurations to determine the configuration and throttle settings which would perform the best. The optimizer was able to determine the idealized throttle setting for each configuration and the resultant total ef-

ficiency, with the baseline configuration having a projected total efficiency of $64.59 \%$ at 
the idealized throttle setting. The best performing configurations were those with the base magnetic ring inwards towards the centerline and the cusp magnetic rings spaced apart. The idealized solution to the best performing configuration, _4i13s, has a projected total efficiency of $66.10 \%$; the poorest performing configuration was _2o31t with a total efficiency of $37.96 \%$ at its optimum. This indicates that, while there is some improvement to be had by changing the magnetic field configuration within the confines of this thruster design, the thruster is theoretically capable of performing quite well in the baseline configuration.

Full thruster testing was performed to determine the operational performance of the thruster. MiXI was operated at a wide range of throttle points, resulting in a wide range of performance parameters. Testing showed the total efficiency of the thruster was capable of reaching $45.9 \pm 24.6 \%$ while having a discharge loss of $173 \pm 43 \mathrm{eV} / \mathrm{ion}$ and a mass utilization efficiency of $0.60 \pm 0.00$. The testing results revealed trends to the operation of the thruster, indicating it may be possible to increase the performance of the thruster with further changes to the throttle values. Specifically, decreasing the propellant flow rate, decreasing the discharge current, and increasing the keeper current have been shown to increase the mass utilization, decrease the discharge loss, and improve overall performance. Decreasing discharge chamber power was shown to increase thruster run time while limiting the amount of excess power increased the time it took to overheat the thruster and allowed for steady state operation of the thruster at some throttle levels.

The testing data was compared to the performance model outputs; the testing results were shown to follow the same trends predicted by the performance models in many regards, but not necessarily the same values. Increasing the beam current was shown to decrease the discharge loss in both the model outputs and the testing results. At a constant discharge loss, and increase in mass utilization efficiency led to an increase in beam current. The consistent trends between the model and the data indicate agreement between the model and data, though limitations to the model and error in the data prevent the sets from matching exactly. 
The creation, analysis, and partial verification of the performance model was enlightening and has led to an increased understanding of the workings of the MiXI-CP-V3 thruster and ion thrusters in general. Operational testing demonstrated the thruster's ability to operate at a wide range of throttle values with varying resultant performance parameters. Ultimately, the work demonstrated in this study has led to an increased understanding to the performance and operation of the MiXI-CP-V3 thruster, furthering the effort to create an efficient, flight capable micro-ion thruster.

\subsection{Future Work}

There is a significant amount of future work to be completed regarding the MiXI thruster. On the computational model side, there are numerous refinements and improvements that can be applied to the models. Replacing the 0-D model with a higher order model will improve the accuracy of the results while still working within the optimizer, resulting in more accurate predictions and performance models. With higher order and more accurate models that more fully capture the spatial interaction of the magnetic fields on the plasma

discharge process, additional potential improvements to the geometry and configuration of the thruster can be determined and tested.

In its current state as a 0-D model, the performance model code only requires the cusp and closed loop magnetic field strengths; a higher order code would utilize the entire magnetic field geometry within its calculations. The design of the magnetic field would become more important as the concerns about the centerline magnetic field strength shape would become apparent in the code and its outputs.

The results from thruster testing were difficult to draw conclusions from due to the inclusion of large amounts of error. Effort was made to create a data acquisition system that would work with the power supplies, thermocouple readers, and Langmuir probe to digitize the data. With more precise data, more accurate conclusions can be drawn. The 
lack of the proper equipment and circuitry prevented the completion of the data acquisition system. It would be important to create a system with substantial accuracy and low noise to monitor the power supply outputs accurately.

The source of numerical error in the data set is largely from the uncertainty of the grid transparency and knowledge of the grid optics. Tests can be performed to experimentally determine the optics of the grid and verify the ion optics within the computational model. These tests should be able to determine the transparency as well as verify the clausing factor calculated by the ion optics submodel.

With the creating of a full data acquisition system, it would be possible to better compare the model to the data sets. It would then be prudent to operate the thruster utilizing Xenon propellant instead of Argon. As was mentioned, the models are designed to perform the calculations for Xenon gas as the identical models are not as well developed for Argon. Using the correct propellant and a precise data acquisition system, it would be possible to accurately compare the model and test data.

It was also discussed that the grids and ceramic isolator subassembly are sub-optimally designed, creating a region inside the discharge chamber where ions and high energy electrons will impact the ceramic isolator, and not the anode wall. This causes unwanted deposition and wear on the isolator, as well as a loss of ions and electrons not accounted for by the test equipment. It would be necessary to redesign the grid and isolator assembly, as well as the location of the anode magnetic ring cusps, to fix the design and increase performance.

All of the design changes to the ion thruster were confined to work within the physical limitations of the MiXI-CP-V3 thruster. With higher order models and more accurate prediction tools, it may be determined that a different thruster geometry and an entirely different magnetic configuration may perform better than the current MiXI design. 


\subsubsection{Model Verification}

It was not possible to verify all portions of the model without code or data sets to compare with. Many thruster examples and data sets were incomplete and it was impossible to completely replicate the problem or results of those thrusters. Verification of portions of the sub-models was possible given some sampled data from the NEXT and NSTAR thrusters, courtesy of Dr. Goebel. The most critical models, the electron temperature sub-model and plasma discharge sub-model, were able to be verified using this data, proving a strong basis for the rest of the models. Given the same set of inputs, the models developed for this thesis were able to determine the electron temperature, internal pressure, average plasma density, discharge loss, discharge current, plasma potential, and confinement factor for the NEXT thruster a accurately for every term.

An attempt was made to use the testing data to verify the performance model. As has been shown in the performance model comparison section (7.2), the data does not fully agree with the model outputs. Identical trends were apparent between the test data and model outputs, but not the same values in all cases. Due to the large amount of error in the testing data, it was not possible to accurately verify the performance model with the results from testing.

Rather than using full operational testing to verify the computational model, a different approach should be considered testing and verifying smaller portions of the code at a time. It would be possible to conduct experiments to test against specific submodels and alter them to operate with the MiXI thruster configuration.

\subsubsection{Model Refinements}

The computational models used within the performance model are not well designed for smaller thrusters and the same code applied to the geometry of a smaller ion thruster does 
not always yield feasible or reasonable results. There are known limitations to the 0-D models [15] and they are not always able to determine the performance correctly for all thruster configurations and all throttle settings. To increase the accuracy, other higher order models must be used.

All of the models described in Section 4 are 0-D, homogeneous, quasi-neutral models with a number other limiting assumptions. It should be expected that the results from the models are not perfect when compared to higher order models and test data. The goal of the models was to provide a level of understanding to the inner workings of the MiXI thruster, and ion thrusters in general, to make predictions about performance and the effects changing the thruster model and operational parameters will have on that performance.

There are many ways in which this model can be improved to achieve more accurate results and more consistent solutions. Many of these improvements require formulating the problems as a 2-D problem with a mesh over the discharge chamber domain and solving the relevant equations in every element. The code and the time required to do this was beyond the scope of this thesis. The way the entire model has been formulated and set up here allows for parts of the performance model to be replaced with higher order models while still interfacing with all of the other parts and working within the optimizer.

The optimizer is a simple optimization around one objective variable. With a further understanding of mission requirements or spacecraft design, it would be possible to reconfigure the optimizer to provide the optimal solution of a thruster given many different sets of inputs.

There are a number of inconsistencies between the models that could stem from any number of sources, but these models are still enlightening in showing the processes working within the MiXI thruster. Specific to the power deposition, the model describes which components of the thruster are heated and how severely. Further refinements to the level of 
detail in these codes would undoubtedly yield more consistent results between the models and accurate results in general. 


\section{BIBLIOGRAPHY}

[1] J. J. Anderson, I. Katz, J. E. Polk, and J. R. Anderson. Numerical Simulations of Ion Thruster Accelerator Grid Erosion. AIAA Joint Propulsion Conference, 7-10 July 2002.

[2] J. R. Beattie. XIPS Keeps Satellites on Track. The Industrial Physicist, 1999.

[3] A. S. Boever, V. Kiim, A. S. Koroteev, L. A. Latyshev, A. I. Morozov, G. A. Popov, Y. P. Rylov, and V. V. Zhurin. State of the Works of Electrical Thrusters in the USSR. International Electric Propulsion Conference, 1991.

[4] J. R. Brophy. Simulated Ion Thruster Operation Without Beam Extraction. AIAA International Electric Propulsion Conference, 18-20 July 1990.

[5] J. R. Brophy. NASA's Deep Space 1 Ion Engine. Review of Scientific Instruments, 2002.

[6] N. G. R. Center. NASA - DS1: How the Ion Engine Works. http://www.nasa.gov/centers/glenn/technology/Ion_Propulsion1.html, 2006.

[7] F. F. Chen. Langmuir Probe Disgnostics. IEEE, 5 June 2003.

[8] R. W. Conversano and R. E. Wirz. CubeSat Lunar Mission Using a Miniature Ion Thruster. University of California, Los Angeles, 2011.

[9] M. T. Domonkos, J. E. Foster, and G. C. Soulas. Wear Testing and Analysis of Ion Engine Discharge Cathode Keeper. Journal of Propulsion and Power, 2005.

[10] C. J. Freitas. Editorial Policy Statement on the Control of Numerical Accuracy, Revision. ASME Journal of Fluids Engineering, 1993. 
[11] R. H. Goddard. The Green Notebooks. The Dr. Robert H. Goddard Collection at the Clark University Archives, 1906.

[12] D. Goebel and I. Katz. Fundamentals of Electric Propulsion: Ion and Hall Thrusters. JPL Space Sciences and Technology Series, 2008.

[13] D. M. Goebel, I. Katz, J. .Ziemer, J. R. Brophy, J. E. Polk, and L. Johnson. Electric Propulsion Research and Development at JPL. AIAA Joint Propulsion Conference, 10-13 July 2005.

[14] D. M. Goebel, J. E. Polk, and A. Sengupta. Discharge Chamber Performance of the NEXIS Ion Thruster. AIAA Joint Propulsion Conference, 11-14 July 2004.

[15] D. M. Goebel, R. E. Wirz, and I. Katz. Analytical Ion Thruster Discharge Performance Model. AIAA, 2006.

[16] D. Griffiths. Introduction to Electrodynamics (3rd Edition). Pearson Education Limited, 1999.

[17] W. M. Haynes and D. R. Lide. CRC Handbook of Chemistry and Physics: A Ready-Reference Book of Chemical and Physical Data. CRC Press, 2010.

[18] D. Knapp. Development, Design, and Test of a Miniature Xenon Ion Thruster (MiXI). Technical report, California Polytechnic San Luis Obispo, 2012.

[19] D. Knapp. Implementation of a 1/4 Inch Hollow Cathode into a Miniature Xenon Ion Thruster (MiXI). Master's thesis, California Polytechnic San Luis Obispo, 2012.

[20] P. R. Lawson, O. P. Lay, K. J. Johnston, and C. A. Beichman. Terrestrial Planet Finder Interferometer Science Working Group Report. JPL Publication, 2007.

[21] J. F. Liu and M. H. Walmer. Thermal Stability and Performance Data for SmCo 2:17 High-Temperature Magnets on PPM Focusing Structures. IEEE Transactions on Electron Devices, 2005. 
[22] H.-S. Mao. Plasma Structure and Behacior of Miniature Ring-Cusp Discharges. PhD thesis, University of California Los Angeles, 2013.

[23] Mathworks. Find Minumum of Constrained Nonlinear Multivariable Function MATLAB fmincon. http://www.mathworks.com/help/optim/ug/fmincon.html, 2015.

[24] Mathworks. Find Minumum of Single-Variable Function on Fixed Interval MATLAB fminbnd. http://www.mathworks.com/help/matlab/ref/fminbnd.html, 2015.

[25] Mathworks. Optimization Options Reference - MATLAB and Simulink. http://www.mathworks.com/help/optim/ug/optimization-options-reference.html, 2015.

[26] MATLAB. version 7.12.0 (R2011a). The MathWorks Inc., 2011.

[27] D. Meeker. Finite Element Method Magnetics, Version 4.2 User's Manual. http://www.femm.info/Archives/doc/manual42.pdf, 2012.

[28] J. L. V. Noord. NEXT Ion Thruster Thermal Model. AIAA Joint Propulsion Conference, 8-11 July 2007.

[29] J. V. Noord. Thermal Modeling of an Ion Thruster. PhD thesis, University of Michigan, 1999.

[30] S. Parker. Thermal Modeling and Validation Testing of a Miniature Xenon Ion Thruster. Master's thesis, California Polytechnic San Luis Obispo, 2013.

[31] M. D. Rayman, T. C. Fraschetti, C. A. Raymond, and C. T. Russell. Dawn: A Mission in Development for Exploration of Main Belt Asteroids Vesta and Ceres. Acta Astronautica, 2006.

[32] P. J. Roache, K. N. Ghia, and F. M. White. Editorial Policy Statement on the Control of Numerical Accuracy. ASME Journal of Fluids Engineering, 1986. 
[33] A. Sengupta, J. R. Brophy, J. R. Anderson, and C. Garner. An Overview of the Results from the 30,000 Hour Life Test of the Deep Space 1 Flight Spare Ion Engine. AIAA Joint Propulsion Conference, 11-14 July 2004.

[34] J. S. Sovey, V. K. Rawlin, and M. J. Patterson. Ion Propulsion Development Projects in U.S.: Space Electric Rocket Test 1 to Deep Space 1. Journal of Propulsion and Power, 2001.

[35] L. Spitzer. Physics of Fully Ionized Gases. Interscience, 1962.

[36] J. R. Taylor. An Introduction to Error Analysis: The Study of Uncertainties in Physical Measurements. University Science Books, 1982.

[37] J. R. Wertz and W. J. Larson. Space Mission Analysis and Design. Microcosm Press, 1999.

[38] R. Wirz. Discharge Plasma Processes of Ring-Cusp Ion Thrusters. PhD thesis, California Institute of Technology, 2005.

[39] R. E. Wirz and D. M. Goebel. Ion Thruster Discharge Performance per Magnetic Field Geometry. AIAA Joint Propulsion Conference, 10-13 July 2006.

[40] R. E. Wirz, D. M. Goebel, C. Marrese, and J. Mueller. Development of Cathode Technologies for a Miniature Ion Thruster. AIAA Joint Propulsion Conference, 20-23 July 2003.

[41] R. E. Wirz, R. Sullivan, J. Przybylowski, and M. Silva. Discharce Hollow Cathode and Extraciton Grid Analysis for the MiCXI Ion Thruster. AIAA Joint Propulsion Conference, 9-12 July 2006.

[42] C. Younger. Thermal Models for a 3cm Miniature Xenon Ion Thruster. Master's thesis, California Polytechnic San Luis Obispo, 2010. 


\section{APPENDICES}

Appendix A

\section{LESSONS LEARNED}

There were many lessons learned throughout the entirety of this thesis, many of them through error and personal experience. This section presents a few of the problematic areas encountered when testing the thruster and will provide some guidelines to prevent damage to, and determine problems with, the thruster.

\section{A.1 Hollow Cathode Assembly}

Assembly of the hollow cathode was a non-trivial task and special care needed to be taken to prevent damage to the ceramic isolators and to maintain electrical isolation between the proper components. It was necessary to not over-tighten any of the components during assembly because the ceramic pieces are brittle and can shatter easily. If any isolators do break apart, the cathode assembly must be taken apart and the broken ceramic piece replaced.

It is necessary that many components of the hollow cathode assembly remain electrically isolated, hence the ceramic isolators. During assembly, the heater and propellant line must be isolated from the cathode base except through the heater circuit. The keeper must be isolated from the chassis and the cathode base entirely. The cathode base must be isolated from the chassis. It is important to assemble the cathode assembly correctly and check the components to maintain electrical isolation to allow the cathode to operate.

\section{A.2 Hollow Cathode Poisoning}

Hollow cathode poisoning refers to the insert installed in the cathode becoming chemically altered due to the presence of moisture and oxygen and no longer able to emit electrons. If 
the cathode is heated in an environment with a significant amount of moisture or oxygen, the cathode insert will chemically react and be rendered inert. If this happens, it will be required to replace the cathode insert, an extremely difficult task as it is a tungsten alloy and difficult to machine.

To prevent poisoning the hollow cathode, it is important to purge the propellant line while the test chamber is pumping down to pressure to remove any atmosphere present in the line. Atmospheric air will be present in the fuel line from the cathode connection to the installed diaphragm valve. It is always necessary to only begin heating the cathode at a base pressure less than $5 \times 10^{-5}$ Torr to ensure the partial pressures of oxygen and water are significantly low. Once testing has completed, it is necessary to wait until the cathode insert has significantly cooled before venting the test chamber and introducing Oxygen and moisture. Depending on the pressure within the chamber, it can take an hour or so before the chamber can be vented. This process can be expedited using an inert gas fill system, such as Nitrogen or Argon, providing a convective mode of heat transfer from the hollow cathode.

One method of checking if the cathode is poisoned is to place an ammeter in line with the positive terminal of the keeper/striker power supply and measure the current as the voltage on the supply is increased. If in atmosphere and cool and the cathode insert is not poisoned, there will be detectable micro-amp readings increasing as the voltage across the keeper increases. If inside the discharge chamber and hot, the micro-amp readings will be greater than when the cathode was cool. If more current is read by the ammeter, into the milliamp range, it is likely there is a short within the system that must be isolated and rectified. 


\section{A.3 Electrical System}

The electrical system was the most problematic portion of setting up and running the thruster. There were many different circuits to feed through the vacuum chamber and integrate between the power supplies and the thrusters. It was necessary to approach the construction of the electrical system intelligently always important to label all of the wires and color code them to ensure they are not connected incorrectly. Attaching one wire in the wrong place can damage the thruster or power supplies and set back the project. It is also prudent to standardize the connectors for when a rewire will become necessary. There are also a number of "floating ground" power supplies that must be realized and connected properly. Without the proper connections, it will not be possible to run the thruster with the beam energized and have a safe test environment.

Before running any tests, it is imperative to check the electrical system through continuity and potential checks. The electrical diagrams must be followed exactly and an ohmmeter should be used to read resistances between components, many of which should indicate no connection as they are electrically isolated. It is also necessary to use a voltmeter to check the potential of thruster components relative to ground and other components. It is possible to apply a small amount of voltage, $15 \mathrm{~V}$, to the anode, keeper, screen grid, and acceleration grid and to measure that voltage at each component. If there is a detectable amount of voltage on other components, it is likely there is a short within the system that must be isolated and rectified.

\section{A.4 Metal Deposition}

Through testing, a significant amount of metal deposition within the discharge chamber was observed. The deposited metal coated every inner surface of the discharge chamber, including the isolating ceramic parts, leading to shorts between components within the 
discharge chamber. The deposited metal also had a tendency to plug the cathode orifice when the cathode was cooled, preventing further tests from being able to function. It was theorized that the deposition was tungstate from the cathode insert that was emitted when the cathode was operated with added heater power and the discharge voltage was above $27 \mathrm{~V}$. For the cathode to operate and sustain plasma generation, it was often required to maintain a small amount of heater power during operation. Through the wide range of throttle settings, there were many test points that had a discharge voltage greater than $27 \mathrm{~V}$. Both of these factors led to a somewhat constant amount of metal deposited on the cathode and discharge chamber.

Every so often, depending of the throttle settings and the duration of tests, it was necessary to remove the thruster from the test chamber for cleaning. The grid assembly needed to be removed from the discharge chamber so the ceramic isolator could be very lightly sanded to remove the metal deposition to fix or prevent shorts. The cathode orifice needed to be inspected and the block broken, with a metal pin or something similar, if present.

On other types of thrusters, such as NSTAR or NEXT, small baffles built into the discharge chamber shield the ceramic isolators from the cathode and prevent the metal deposition and shorting between components. These thrusters also are able to operate without any added heater power, limiting the amount of deposition that occurs. While it was not possible to build shielding for the ceramic isolators, they should be considered for future versions of MiXI. 
Appendix B

\section{VACUUM CHAMBER OPERATING PROCEDURES}

The following sections describe the setup and operation for the "Minimum Atmospheric eXperimentation" (MAX) high vacuum chamber at Cal Poly’s Space Environments Lab.

\section{B.1 MAX Vacuum System Initial Setup and Safety Checks}

1. Ensure that all service panels of the vacuum chamber base enclosure are closed and secured.

2. Ensure that all the vacuum control panel toggle switches are in the "off" position (down).

3. Check that the $120 \mathrm{~V}$ surge protector powering the service panels is plugged in.

4. Check that the 3 phase $240 \mathrm{~V}$ source is plugged in to an appropriate, working plug.

5. Flip the breaker in the wall to the "On" position (up).

6. Open the ball valve to the pressurized shop air line.

Note: Check that the pressurized air regulator reads between 70-75 psi.

7. Turn on the Main Power switch on the vacuum control panel.

8. Turn on the Cryogenic Temperature indicator.

9. Turn on the Granville-Phillips 316 Vacuum Gauge controller.

Note: The Convectron Gauge 2 (CG2) indicates the cryogenic pump line pressure in Torr.

Note: The Convectron Gauge 3 (CG3) Indicates the chamber pressure in Torr. 


\section{B.2 Cryogenic Vacuum System Setup}

1. Check that the 3 phase $240 \mathrm{~V}$ power line from the Cryogenics $1020 \mathrm{R}$ compressor to the plug on the vacuum chamber base enclosure is connected.

2. Verify that the supply and return lines from the compressor to the cryogenic pump are connected properly.

3. Verify that the cold head power line from the compressor to the cryogenic pump is attached.

4. Flip the Cold Head and Compressor switches on the front of the compressor to the "on" position.

Note: The compressor will not engage at this point as it is operated from the vacuum control panel.

5. Verify that the supply gauge on the rear of the compressor reads between 275-290 psi and the return pressure is operating in the non-red regions of pressure (XXX determine the pressures that this is).

Note: The pressure may not read correctly or at a steady state until the compressor has been running. After the compressor has been operating for a few minutes (following the correct operating procedures listed below), if the pressures are still not at the correct operational pressures, consult the compressor manual for changing the helium pressure in the system. Helium can be added or removed during operation.

\section{B.3 Experimental Loading Procedure}

1. To raise the lid of the chamber, verify that the internal pressure from CG3 reads about 760 Torr (1 atm).

Note: If the pressure is less than this, turn on the Vent Switch until the internal pressure reaches 760 Torr. 
2. Raise the chamber lid using the hoist control.

Note: There are come cases when the bell jar will become stuck with the lid as it is raised. It is important to not let this fall and crack; keep the lid and jar lowered and carefully unstick the lid from the top seal.

3. Remove the glass bell jar if required.

Note: Place the bell jar on a clean and debris free surface.

4. Install the experimental components according to the goals of the given test.

Note: Ensure that all materials have acceptable outgassing levels to avoid contamination of the chamber and the experiment.

5. Check the bell jar gaskets to ensure a light but complete coating of vacuum grease and a good seal with the lower metal plate.

Note: If the chamber has not been cleaned in a while, wipe down the inside of the bell jar, the gaskets, and the gasket mounting surfaces with water and Kim-wipes. Then apply a light coating of vacuum grease to the gaskets.

6. Ensure that all installed components do not interfere with the vacuum chamber seals or the gate valve.

Note: The gate valve will raise approximately 6 inches when actuated. It is imperative that all components within the vacuum chamber will not interfere and fall under the gate valve when it is raised and lowered.

7. Lower the chamber lid using the hoist control.

8. Check the upper gasket to ensure a uniform seal.

\section{B.4 Chamber Roughing Procedure}

Roughing down the chamber involves taking the bulk of the air out of the vacuum system. This process can only take place with the lid lowered and the bell jar in place.

1. Turn on the Mechanical Pump switch on the vacuum control panel. 
2. Turn on the Chamber Rough Valve to begin pumping down the chamber.

Note: The chamber is capable of reaching pressures as low as $8 \times 10^{-3}$ mTorr after 1-2 hours of pumping.

\section{B.5 Cryogenic System Procedure}

The cryogenic system can be cooled down and set up without the chamber being pumped down. This is a lengthy process and it is advisable to complete this prior to the time of intended use.

1. Turn on the Mechanical Pump switch on the vacuum control panel.

2. Turn on the Rough Interlock Switch on the vacuum control panel.

3. Turn on the Cryo Rough Valve on the vacuum control panel.

Note: Air will now begin to pump out of the lines running between the gate valve and the cryogenic pump.

4. Monitor the 375 Vacuum Gauge Controller 2 to ensure the pressure is falling.

5. Once the CG2 reads the pressure is below $150 \times 10^{-3}$ Torr (150 milliTorr), you may turn on the cryogenic compressor.

Note: It is preferential for the cryogenic compressor to begin the process at as low of a pressure as possible. Engaging the compressor when the pressure is less than 50 mTorr has shown a reduction in required operating time.

6. Verify that the compressor and cold head are operating: the compressor will start running and observing the cold head at the base of the cryogenic pump and ensure it is spinning in a clockwise direction.

7. Monitor the cryogenic pump temperature gauge and verify that the temperature begins to fall.

Note: The temperature will begin to fall very slowly until it reaches a base operating temperature of $10-20 \mathrm{~K}$. 
8. When the temperature gauge reaches a value less than $150 \mathrm{~K}$, it is possible to turn off the mechanical pump. In this order: close the Cryo Rough Valve, Rough Interlock, and Mechanical Pump switches on the vacuum control panel.

Note: The pressure in the cryogenic pump lines, indicated by CG2, must remain below 150 mTorr throughout the entire cooling process. The pressure may fluctuate noticeably but only momentarily when these switches are flipped. If the pressure rises significantly, the compressor and cryogenic pump should be shut off and allowed to return to ambient temperature before troubleshooting can begin.

9. Monitor the temperature and pressure gauges through the cooling process until the cryogenic pump temperature reaches $10-20 \mathrm{~K}$.

Note: This process can take from 1.5-3 hours depending on starting pressure and compressor helium levels.

Note: If the required temperature is not being reached, check the supply and return pressures on the compressor and fill with helium if required.

\section{B.6 Experimental Chamber Vacuum Operating Procedures}

General description of how to operate the vacuum chamber for a typical operation.

1. Follow the Chamber Roughing Procedure to pump the vacuum chamber to low vacuum.

2. Once low vacuum is achieved, follow the Cryogenic System Procedures to prepare the cryogenic pump.

Note: Once the temperature of the cryogenic pump is at the appropriate level, proceed to the next step.

3. In this order: turn off the Chamber Rough Valve and the Mechanical Pump switches.

4. Flip the Pressure Interlock switch to the On position.

5. Flip the Gate Valve switch to the "on" position. 
Note: The gate valve will open and raise approximately 6 inches when this happens. If this is not the case, verify that the shop air pressure is sufficient and refer to the previous sections to ensure the proper switches have been toggled.

Note: If an object falls into the gate valve, immediately turn off the compressor. Refer to the tips and tricks for turning the system off and warming the cryo pump and refer to the user's manual to remove the object and repair the cryo pump.

6. Verify that the pressure reading in the chamber (CG3) has fallen to 0 on the gauge; this should happen rapidly.

Note: The convectron gauge can only read to pressures as low as $1 \times 10^{-3}$ Torr and will display 0 when below this value.

7. Only once the pressure displays 0 on CG3, turn on the Ionization Gauge (IG) on the Granville-Phillips 330 Vacuum Gauge Controller.

Note: Turning on the Ionization Gauge before the chamber pressure gauge reaches 0 mTorr can result in permanent damage to the ion gauge.

8. Experimentation can commence once the required base pressure has been reached.

Note: The cryogenic pump is capable of reaching pressures as low as $5.4 \times 10^{-7}$ Torr. Improvements to this may be possible but with significant and costly modifications to the system.

\section{B.7 Experimental Unloading Procedure}

1. Turn off the Ionization Gauge on the Granville-Phillips 330 Vacuum Gauge Controller.

2. Switch off the Gate Valve.

Note: The gate valve will now close and lower by approximately 6 inches. If objects are in the way or if something has fallen into the system, refer to the Tips and Tricks section after this to remedy the problem. 
3. Switch off the Pressure Interlock switch.

4. Switch off the Compressor switch to turn off the cryogenic pump system and the compressor.

Note: At this point, if it is intended to use the cryogenic system again immediately, leave the compressor on. The cryogenic system will stay cold and under vacuum.

5. Turn on the Vent Valve switch to vent the chamber. Vent the chamber to atmospheric pressure (760 Torr).

6. Raise the bell jar lid with the hoist control.

7. Remove the bell jar if necessary.

Note: Remember to place the bell jar on a clean, debris free surface.

8. Remove experimental equipment.

9. Replace bell jar on chamber for safety.

10. Follow the Chamber Roughing procedure to rough the chamber to low vacuum again for storage.

Note: It is better for the pumps and chamber to be kept clean and under low vacuum when not in use for long periods of tome.

\section{B.8 Tips and Tricks}

This section contains troubleshooting tips and performance tricks learned while operating the vacuum chamber and cryogenic pump.

- If the desired base pressure cannot be reached, the following is a prioritized list of leak locations. Check these one at a time to find the source of leaks.

1. Tighten or close off any fuel lines.

2. Remove the bell jar and clean all the seals, apply new vacuum grease as needed. 
3. Run the Ionization Gauge through a DGAS cycle, the IG may be displaying a different pressure than what it actually is due to contaminants.

4. Remove the feedthrough's and clean them with acetone, clean the O-rings with water, wipe down the feedthrough caps and interfaces with acetone. Allow the acetone to evaporate for a few minutes before reinstalling the O-rings, caps, and feedthroughs.

5. Run the Cryogenic System through a regeneration cycle following the procedure listed below.

Note: using a helium leak detector may help find leaks but can be far more time consuming than simple maintenance if the leak is not discovered right away.

- If the compressor will not turn on when the switch is activated, verify power to the compressor and that the cover limit switch is depressed. The compressor will shut down if this switch is not actuated.

- If the compressor turns on and only runs for a few seconds before switching off, check the window on the Cryogenic Chamber's Cold Head. The head should rotate clockwise without any grinding or seizing. If the head moves less than one rotation clockwise or counterclockwise while making a grinding noise, the power to or within the compressor may not be in phase. Try plugging the compressor into another outlet or contacting the facilities electrician. If the compressor still does not function and the source power is confirmed correct, the problem is internal to the compressor. Consult the compressor's user manual for assistance.

- If a faster or wire interferes with the operation of the gate valve and the valve cannot close, it will be necessary to follow the Cryogenic Pump Regeneration Procedure below. The cryogenic pump must be warmed up slowly to avoid contamination and allowed to reach ambient temperature before the chamber can be vented and the interfering object removed. 


\section{B.8.1 Ionization Gauge DGAS}

The Ionization Gauge has a DGAS switch that will heat up the filament within the gauge and clean any contaminants within, improving the accuracy of the readings from the gauge.

1. When the base pressure within the chamber is less than $1 \times 10^{-4}$ Torr (following the Chamber Roughing and Cryogenic System Procedures), press the DGAS switch on the Granville-Phillips 330 Vacuum Gauge Controller.

Note: The heating filament will turn on; allow this to heat for 2-3 minutes while the cryogenic pump operates and the gate valve is open.

2. Turn off the DGAS switch.

3. Allow the filament to cool for a few minutes before resuming normal operation of the Ionization Gauge.

\section{B.8.2 Cryogenic Pump Regeneration Procedure}

If the desired base pressure cannot be reached with the compressor and cryogenic pump, there may be contaminants in the cryogenic fluid or helium supply. This can be cleaned to a degree by running the system through a regeneration cycle.

1. Follow the Chamber Roughing Procedures and Cryogenic System Procedures.

2. Once the temperature of the cryogenic system has reached $10-20 \mathrm{~K}$, turn on the Pressure Override switch.

3. Open the Gate Valve switch.

Note: The gate valve will open at this time, ensure no objects interfere with or fall into the valve.

4. Turn the compressor off.

5. Turn the roughing pump on.

6. Open the Chamber Roughing valve. 
Note: The gate valve will close at this point.

7. Leave the roughing pump operating while the cryogenic system warms up to ambient temperature.

Note: This process can take from 3-5 hours.

8. In this order: switch off Gate Valve then the Pressure Override.

9. In this order: close the Chamber Roughing valve and turn off the Roughing Pump 
Appendix C

HOLLOW CATHODE STAND ALONE TESTING PROCEDURES

The following section describes the procedures and components required for operating the hollow cathode in Cal Poly's Minimum Atmospheric eXperimental (MAX) vacuum chamber. A list of the required materials and propellant system components are listed in Table C.1 and a list of the required electrical equipment is provided in Table C.2.

Note: It is recommended the user to be prepared and practiced in lighting the hollow cathode prior to full thruster testing. Extra time spent heating the cathode and attempting to ignite the plasma can lead to excessive heating and limit the operational time of the thruster. 
Table C.1: Hollow Cathode System Bill of Materials

\begin{tabular}{|l|c|l|}
\hline Component & Amount & Description \\
\hline Hollow cathode & 1 & Provided assembly by JPL \\
\hline Propellant supply & 1 & Research grade (99.999\% pure Xenon or Argon) \\
\hline Propellant isolator & 1 & $\begin{array}{l}\text { Electrically isolates propellant lines and fuel } \\
\text { tank from cathode }\end{array}$ \\
\hline Diaphragm valve & 1 & Swagelok SS 4H \\
\hline Needle valve & 1 & VACOA MV-25 \\
\hline Flow meter & 1 & Omega FMA-A2300 \\
\hline Pressure regulator & 1 & Smith, High purity \\
\hline Fuel line pipe sections & - & $1 / 16 "$ - 1/4" Swagelok aluminum pipe, as needed \\
\hline Discharge chamber & 1 & Electrically isolated metal tube \\
analog & $1+$ & J,K Type \\
\hline Thermocouple wire & $1+$ & J,K Type to match \\
\hline Thermocouple Reader & &
\end{tabular}

Table C.2 is an annotated list of the power supplies and their ratings used in the operation of the hollow cathode. 
Table C.2: Hollow Cathode Electrical System Bill of Materials

\begin{tabular}{|l|c|l|}
\hline Component & Amount & Description \\
\hline Cathode Heater & 1 & HP 6038A \\
\hline Cathode Keeper & 1 & Agilent N5747A \\
\hline Discharge/Anode & 1 & Sorensen XG 1700 300-5.6 \\
\hline Screen/Striker & 1 & Glassman FC1P120 \\
\hline High Voltage Diode & $2+$ & \\
\hline High Current Diode & $2+$ & \\
\hline Wires & - & As needed, inside and outside vacuum chamber \\
\hline
\end{tabular}

\section{C.1 Pre-Setup Procedures}

1. Locate the equipment listed in Table C.1.

2. Clean the cathode, the interior of the vacuum chamber, and all items to be placed in the vacuum chamber with acetone and "Delicate Task Kim-Wipes".

Allow 4-6 hours for the acetone to fully dry and evaporate to reduce the risk of contaminating the cathode. It is necessary to wear powder-free rubber gloves when handling the equipment after it has been cleaned.

3. Optional: Run the vacuum chamber through a regeneration cycle B.8.2

\section{C.2 Hollow Cathode Assembly and Loading}

Note: Read the Experimental Loading procedures prior to loading the cathode into the vacuum chamber B.3.

1. Remove the glass bell jar; place the jar onto a clean, debris free surface while the experiment is installed. 
2. Identify the electrical feedthroughs, thermocouples, and propellant line that will be used.

3. Install the Hollow Cathode in the vacuum chamber.

4. Using the available propellant lines, connect the lines from the propellant feedthrough through the Propellant Isolator and to the Hollow Cathode.

With the cathode, propellant isolator, and fuel lines are installed, the chamber should looks similar to the setup in Figure C.1.

5. Place the anode analog outside the exit of the hollow cathode within the vacuum chamber. Ensure the cathode and anode are not in contact.

6. Attach the thermocouple to the cathode base and/or the anode analog. These will be used to monitor the temperature of the components.

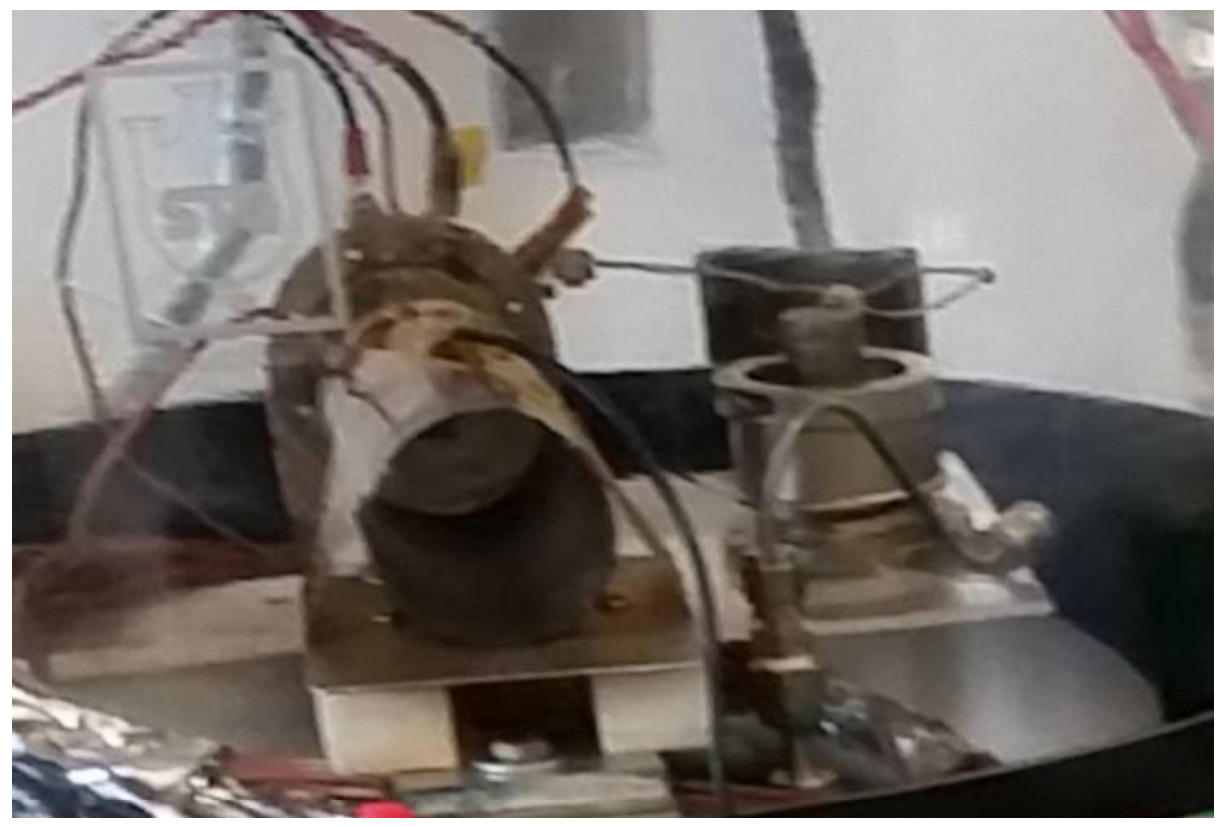

Figure C.1: Hollow Cathode Stand Alone Setup

\section{C.3 Propellant System Assembly}

This section will detail the assembly of the propellant system external to the vacuum chamber. The propellant system will be setup according to the schematic in Figure C.2. 


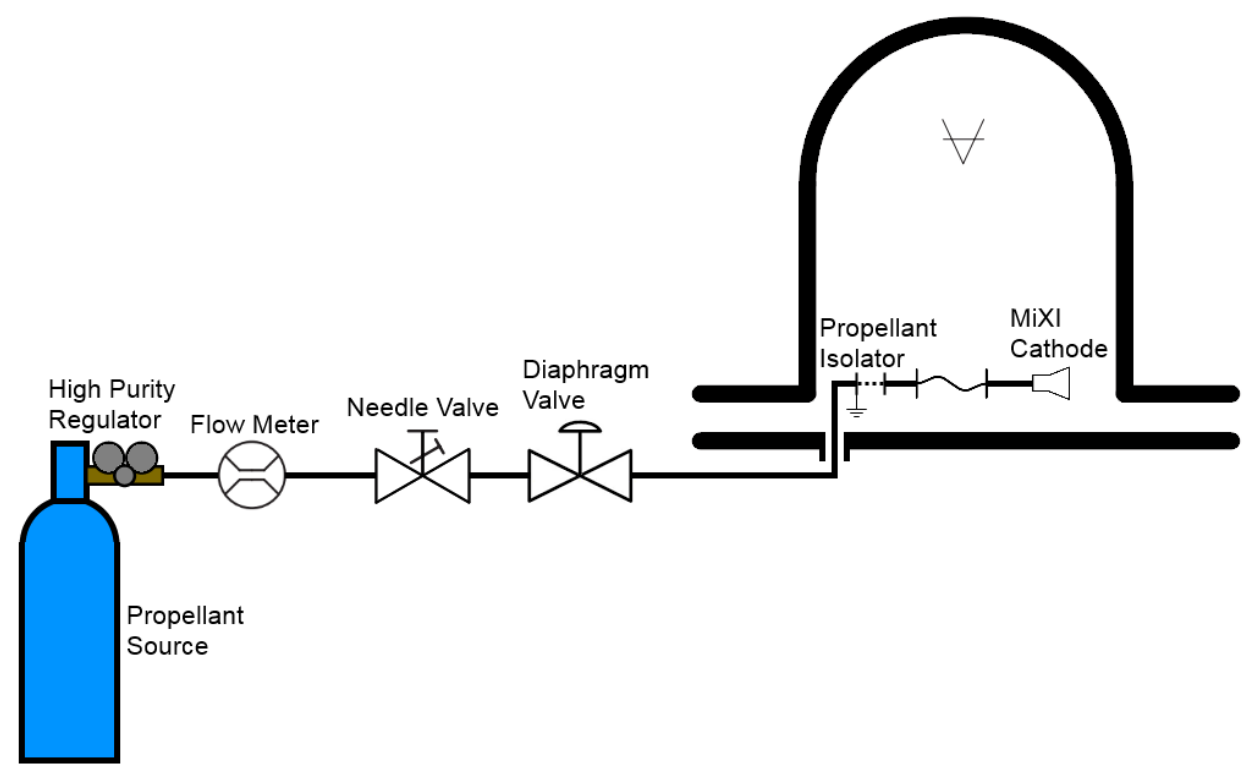

Figure C.2: Propellant System Setup Schematic

1. Insert and tighten the Smith regulator to the propellant source tank.

2. Roughly layout the components outside the vacuum chamber according to the schematic in Figure C.2.

3. Using 1/4" Swagelok pipe sections, connect the end of the feedthrough to the downstream connector of the Diaphragm Valve.

It is generally beneficial and easier to install the system starting from the feedthrough on the vacuum chamber to the propellant tank.

4. Connect the upstream connector on the Diaphragm Valve to the downstream connector of the Omega Flow Meter.

Using the DC adapter, plug in the flow meter to a power outlet; the display should read '00.00'.

5. Connect the upstream connector of the Omega Flow Meter to the needle valve.

6. Connect the other end of the needle valve through whatever piping is required to the open end of the pressure regulator. 


\section{C.4 Electrical System Assembly}

This section describes the setup of the electrical system required to operate the hollow cathode in a stand along capacity. The required power supplies are listed in Table C.2 and the electrical schematic for the system is displayed in Figure C.3. The wires installed within the vacuum chamber should be clean and low outgassing wires designed for use within a vacuum chamber.

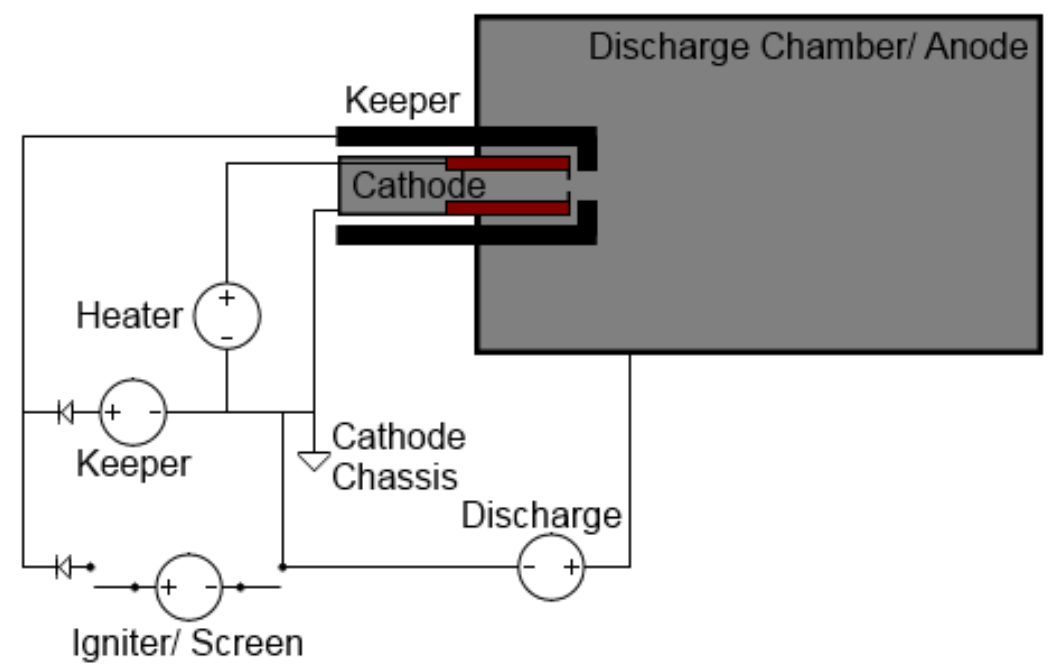

\section{Figure C.3: Hollow Cathode Stand Alone Schematic and Electrical Diagram}

1. Ensure the power supplies are powered off prior to setup.

2. Attach the appropriate wires to terminals of the cathode; connect to the heater, keeper, and cathode common terminals.

3. Attach a grounding wire to the cathode common terminal. This can be accomplished within or exterior to the vacuum chamber.

4. Connect a wire to the discharge chamber analog. 
Note: The discharge chamber analog might not have ring or slot connectors and it may be necessary to strip and attach the exposed wire to the discharge chamber with Kapton tape.

5. Connect the free ends of the heater, keeper, cathode common, and discharge wires to their respective feedthroughs in the vacuum chamber.

Note: If the feedthroughs are located in the lid of the chamber, it will be necessary to install the bell jar before connecting the wires to the feedthroughs.

6. Secure the wires so they will not interfere with the operation of the gate valve.

7. Exterior to the vacuum chamber, attach the feedthrough terminals to their respective power supplies.

8. There are diodes installed between the keeper and striker power supplies that protect the power supplies from the high voltage and current present from the other power supplies. These diodes are important and cannot be ignored. The high voltage diode should be placed downstream of the high current diode (closer to the feedthrough terminal). It is advisable to have extra diodes as the current during operation can exceed the limits of the high voltage diode and cause it to fail.

9. Perform a connectivity test between all components.

The meter should indicate connectivity between the cathode common/ground and the heater terminal. This reading should be around $1 \Omega$.

The meter should indicate electrical isolation between the cathode common node, the keeper, and the discharge chamber analog.

\section{C.5 Hollow Cathode Operating Procedure}

1. As the chamber is pumping down, open all valves in the propellant system up to, but not including, the regulator valve. This places the propellant lines under the same vacuum as the chamber and removes any atmosphere from the propellant line. 
2. Slowly open the regulator valve on the propellant tank to allow propellant gas to flow through the lines. This purges the lines and removes any Oxygen or moisture within the system that will poison the cathode.

Note: The downstream gague on the regulator will indicate a negative pressure reading. Slowly open the valve to increase the flow until there a small but discernible pressure reading on the downstream gague of about -20 psi.

3. Close the needle valve and diaphragm valve in the propellant line system. During testing, these will regulate the flow to the hollow cathode.

4. Once the required base pressure of $\leq 5.0 \times 10^{-6}$ Torr is reached, open the diaphragm valve while keeping the needle valve closed. The pressure will increase slightly and stabilize. The propellant flow is now completely controlled by the needle valve.

5. Turn on the heater power supply and set it to $6 \mathrm{~A}$ current controlled.

Note: Do not exceed 6A as this can damage and break the heater, requiring it to be replaced.

6. Wait for the cathode insert to heat to the appropriate level. The insert must reach the required temperature to begin emitting electrons $(\sim 1100 \operatorname{deg} C$ for $B a O-W)$; the orifice will heat and change color to a bright yellow-white color. This process usually takes between 5-10 minutes.

7. Increase the propellant flow rate using the needle valve. Set the flow rate to a value over $6 \mathrm{SCCM}$ on the reader.

8. Turn on the keeper power supply; it should automatically be set to voltage controlled. Set the current limit to an appropriate value for testing ( 1A). Slowly increase the keeper supply voltage until the cathode strikes. This will be apparent when plasma is visibly generated and the keeper supply switches from voltage controlled to current controlled and draws the full current set by the supply.

9. If the maximum voltage of the keeper supply is reached and the cathode has not struck, it will be necessary to use the screen/igniter power supply to light the cathode. 
Set the keeper power supply voltage to a smaller value $(\sim 50 \mathrm{~V})$ while leaving the current limit the same. Turn on the igniter power supply and set the current limit to maximum $(\sim 120 \mathrm{~mA})$. Slowly increase the voltage on the igniter power supply until the cathode strikes.

Note: Depending on the condition of the cathode, this can sometimes take up to $600 \mathrm{~V}$ to strike.

10. Once the cathode strikes, the igniter supply can be shut off.

11. Turn on the discharge power supply. Set desired current value.

12. Slowly increase the discharge power supply voltage. There will be an arc and the plasma will drive current through the discharge power supply.

13. Once both power supplies are stable, the propellant flow rate can be adjusted to the desired value.

The cathode can be operated for extended periods of time without worry of overheating; there are no temperature sensitive components on the cathode assembly. Once the plasma discharge is stable, it is possible to reduce the added heater power to the cathode insert, though it may not always possible to have the cathode operate in a self heating state.

To turn off the cathode, turn off the discharge power supply, then turn off the keeper power supply; also turn off the heater power supply if it was still in use. Allow the cathode to cool for an extended period of time before opening the chamber. Adding moisture and Oxygen to the chamber can poison the cathode insert.

\section{C.6 Troubleshooting Tips and Tricks}

- If the cathode cannot strike as the igniter voltage is increased, increase the propellant flow rate to the cathode. The more fuel and the higher local pressure at the cathode orifice will facilitate ignition. 
- There should be no detectable current on the keeper/igniter power supplies as the voltage is increased when trying to strike the cathode. If there is a detectable amount of current, it is likely there is a short in the cathode assembly that must be fixed before the cathode will strike.

- If the cathode still cannot be struck with the igniter voltage maxed out, the cathode insert may be poisoned. One method of checking if the cathode is poisoned is to place an ammeter in line with the positive terminal of the keeper/striker power supply and measure the current as the voltage on the igniter supply is increased. If in atmosphere and cool and the cathode insert is not poisoned, there will be detectable micro-amp readings increasing as the voltage across the keeper increases. If the cathode insert is hot, the micro-amp readings will be greater than when the cathode was cool. If zero current is detected, it is likely the cathode insert is poisoned and professional help will be required. If more current is read by the ammeter, into the milliamp range, it is likely there is a short within the system that must be isolated and rectified.

- If the cathode is not used for an extended period of time, there may be a small layer of oxidation on the cathode orifice that can make the cathode more difficult to strike. If the aforementioned tips for striking the cathode don't work, it may be required to allow the cathode to heat for 30 minutes or more; the cathode may be easier to strike after an extended period of heating.

- If the cathode still cannot be struck, professional help may be required. 
Appendix D

\section{MIXI FULL TESTING PROCEDURES}

The following section describes the procedures to install and operate the MiXI-CP-V3 ion thruster in the Cal Poly Minimum Atmospheric eXperimentation (MAX) chamber. The setup and procedures for installing and operating the hollow cathode is provided in Appendix $\mathrm{C}$; a table describing the additional required equipment is provided in Table D.1.

Table D.1: MiXI Additional Setup Equipment

\begin{tabular}{|l|c|l|}
\hline Component & Amount & Description \\
\hline Discharge Chamber & 1 & Constructed following procedures in Section 2 \\
\hline $\begin{array}{l}\text { Discharge chamber } \\
\text { mounting hardware }\end{array}$ & - & Isolating and mounting hardware \\
\hline $\begin{array}{l}\text { Discharge/Anode } \\
\text { Power Supply }\end{array}$ & 1 & Sorensen XG 1700 300-5.6 \\
\hline $\begin{array}{l}\text { Screen/Striker Power } \\
\text { Supply }\end{array}$ & 1 & Glassman FC1P120 \\
\hline $\begin{array}{l}\text { Acceleration Grid } \\
\text { Power Supply }\end{array}$ & 1 & Glassman FC1P120 \\
\hline $\begin{array}{l}\text { Neutralizer CTFC } \\
\text { Supply }\end{array}$ & 1 & HP 6263B \\
\hline Wires & - & As needed, inside and outside vacuum chamber \\
\hline
\end{tabular}




\section{D.1 MiXI Loading Procedure}

Note: Refer to Experimental Loading Procedures B.3 prior to installing MiXI into the chamber. Refer to Hollow cathode installation and operational procedures located in Appendix $\mathrm{C}$ for information on the setup of the hollow cathode when necessary.

The cathode chassis and anode were originally designed to interface through a series of all-thread screws and ceramic isolators, attaching the anode base directly to the cathode chassis. Over the years, the cathode has been repaired and replaced and the chassis and anode base no longer directly attach. In this thesis, the anode was attached to a custom bracket with ceramic isolators and the bracket was attached to the test stand base. The spacing between the cathode and anode was achieved through careful positioning of the anode bracket in the test chamber.

1. Assemble the anode, magnets, and thruster grids as described in the System Configuration Section 2.

2. Install the hollow cathode assembly as described in Appendix C. The following steps assume the installation of the hollow cathode has been completed.

3. Place the Keeper Isolation Sheath around the tip of the keeper.

4. Attach the discharge chamber and grid assembly to the test stand base inside the vacuum chamber.

Place the discharge chamber opening around the keeper and keeper isolation sheath. Adjust the position of the discharge chamber to the desired location. Align the keeper and discharge chamber coaxially.

5. Install the neutralizer CTFC outside the exit of MiXI. Ensure the CTFC does not contact any part of the MiXI thruster.

Note: The neutralizer should be placed downstream the grid aperture, centered along the ion extraction area, and a few centimeters away from the accelerator grid. 


\section{D.2 Propellant System Assembly}

There are no additional components required to install the MiXI thruster compared to the hollow cathode stand alone propellant system setup. The procedures describing the setup are located in Appendix C.

\section{D.3 Electrical System Assembly}

This section will describe the setup of the electrical system required to operate MiXI. An electrical component diagram is provided in Figure D.1. Be sure to shut down all of the power supplies when not in use. Insulate all exposed terminals and diodes to prevent electrical shock. The wires installed within the vacuum chamber should be clean and low outgassing wires designed for use within a vacuum chamber.

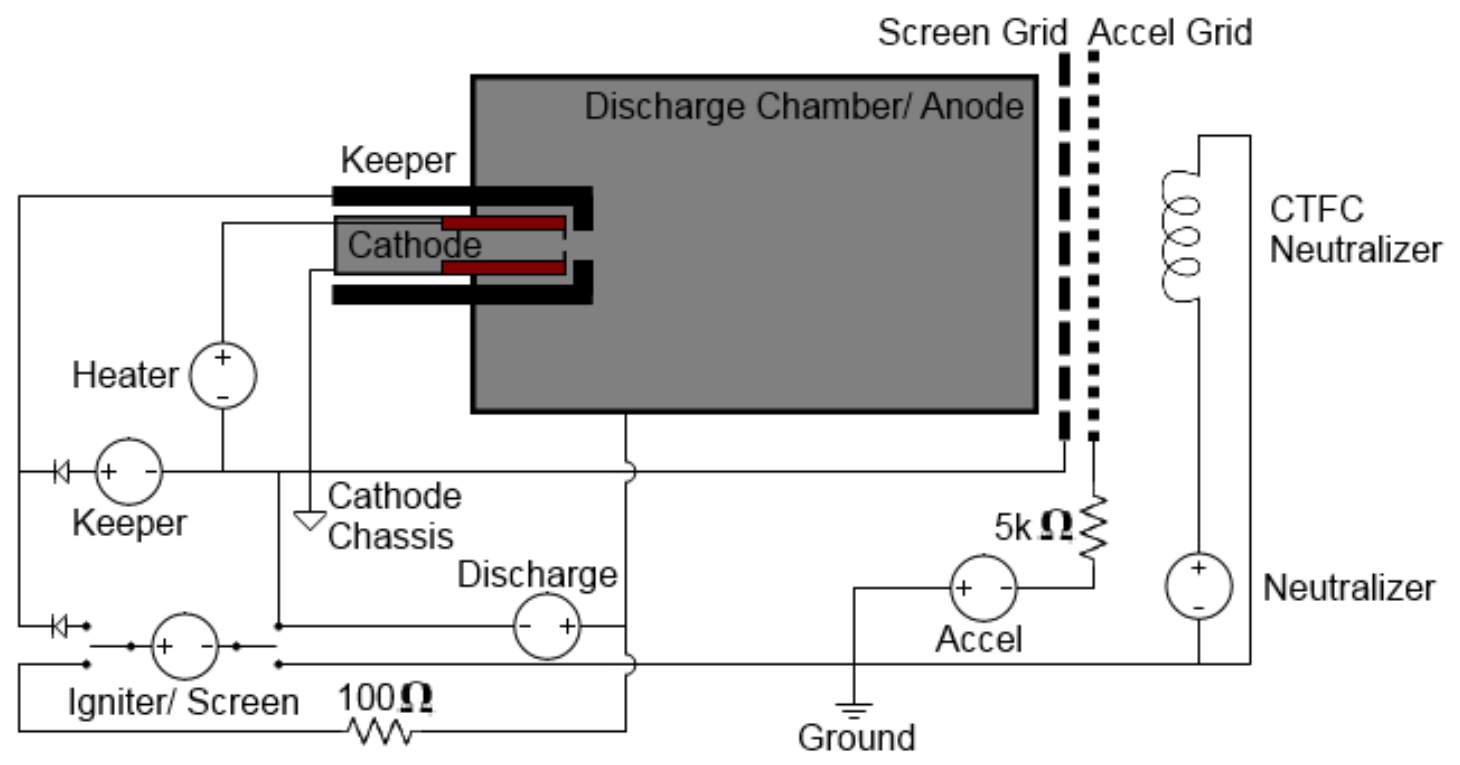

Figure D.1: Electrical Schematic and Component Diagram of MiXI

There are three power supplies that must have a floating ground relative to earth ground: the heater, keeper, and discharge power supplies. They must be installed using power 
supply isolators to decouple the ground and negative terminal of the power supplies. The acceleration grid power supply must also be installed in a way to provide negative polarity potential to the acceleration grid.

1. Setup the Keeper and Heater supplies according to the Hollow Cathode Electrical Setup Procedure in Appendix C.4.

2. Within the vacuum chamber, attach the discharge/anode lead to the discharge chamber.

Note: If the feedthroughs are located in the lid of the chamber, it will be necessary to install the bell jar before connecting the wires to the feedthroughs.

3. Attach a wire between the screen grid and the feedthrough node for the cathode chassis.

4. Attach a wire between the acceleration grid and an unused feedthrough.

5. Attach a wire from the discharge chamber to an unused feedthrough.

6. Connect the neutralizer CTFC to an unused feedthrough. The polarity on the CTFC is irrelevant.

7. Exterior to the vacuum chamber, connect the acceleration and neutralizer power supplies to their respective component feedthroughs.

Note: Connect the negative terminal of the acceleration power supply to the feedthrough and the positive to ground. Install a $5 \mathrm{k} \Omega$ resistor between the power supply and the feedthrough.

8. Install a method of switching the connections of the high voltage screen/igniter power supply. Both terminals of the power supply must be switchable, as shown in Figure D.1.

9. Install a $100 \Omega$ resistor between the positive terminal of the screen power supply and the other end of the discharge chamber wire feedthrough. Connect the negative terminal of the screen power supply to ground. 
10. Attach the positive terminal of the discharge power supply to the discharge chamber wire feedthrough.

\section{D.4 MiXI Operational Procedure}

Follow the Hollow Cathode Operating Procedure in Appendix C.5 for information on the steps required to operate the hollow cathode. Operation of the MiXI thruster assumes the cathode is able to be struck following the Hollow Cathode Operating Procedures.

1. Follow the procedures in Appendix C.5 to strike the cathode.

2. Once the cathode has been struck and plasma has been formed, turn off the igniter power supply, if used. Reduce the added heater to the appropriate level.

3. Turn on the discharge/anode power supply and adjust the current until the desired discharge current has been reached.

4. Slowly close the needle valve on the propellant line until the desired propellant flow rate is reached.

5. Switch the igniter power supply to the screen circuit. Set the screen supply to the desired voltage with maximum allowance for current (typically 125 milliAmps).

6. Turn on the acceleration grid power supply and set to the desired voltage.

Note: It is typical to see some small amounts of electrical discharge from the beam plasma to the vacuum chamber test stand. The beam has not been neutralized and charged particles are interacting with the system ground. To negate this, turn on the neutralizer CTFC before the screen and acceleration grid power supplies.

7. Turn on the CTFC neutralizer cathode and set the current to the value specified in the test.

The MiXI thruster should now be operating fully with all required power supplies functioning with minimal to no electrical discharge in the vacuum chamber. 
8. Allow MiXI to operate steadily until the test is completed or until the thermocouple indicates the temperature of the anode base is greater than $300 \operatorname{deg} C$.

Note: This can occurs after 5-10 minutes depending on the throttle settings. Various throttle settings with less discharge current may take longer to overheat or be able to maintain steady state operation. The SmCo magnets may be permanently damaged if their temperature exceeds $325 \operatorname{deg} C$.

Once the tests have been completed, turn off the power supplies in this order: Acceleration Grid, Screen Grid, Neutralizer, Discharge/Anode, Keeper. Close the Needle Valve and diaphragm valve in the propellant line to stop propellant flow. Allow the thruster to cool for a until the cathode and apparatus reach a temperature less than $50 \operatorname{deg} C$, if planning to vent the vacuum chamber. If more tests are to be conducted, the thruster can be operated again once the user has deemed the thruster cool enough to allow for time to complete the required tests. Once the tests are complete, refer to the Experimental Unloading Procedures in Appendix B.7.

\section{D.5 Troubleshooting Tips and Tricks}

- If the thruster is not able to strike and form the proper current paths, perform electrical connectivity tests between the components to check for shorts between components.

- After the screen and acceleration grids are energized, arcing may occur between them. This may introduce error into any experimental data that is being collected. If excessive arcing occurs, the spacing between the grids may be too close for the grid potentials applied. Consider inspecting the grids for damage and warping from heat and arcing; it may be necessary to clean the grids or adjust the spacing the grids further apart to prevent further arcing and damage. 
- The energy from the hollow cathode heater is transferred to the MiXI thruster. The longer the heater is left powered prior to striking the cathode, the hotter MiXI will be and the operational time will become limited.

- The switch installed from the screen/igniter power supply to the system should be a high voltage rated switch. The housing for the switch must be electrically isolated and safe for the operator. 
Appendix E

\section{GRID CONVERGENCE STUDY AND ERROR ANALYSIS}

The following section describes the steps taken to perform a grid convergence study of the meshes generated by FEMM for use in this thesis. A grid convergence study was performed to verify that the meshes used and the cell sizes yielded results that are sufficiently accurate. The methods and procedures are taken from the "Journal of Fluids Engineering Editorial Policy Statement on the Control of Numerical Accuracy" [32, 10]. While normally used to verify the results used for CFD purposes, the methods are applicable to verify that the meshes generated from FEMM will have sufficiently small error.

The basic requirements to control numerical accuracy state the solutions to newly developed code must be verified with previous code verification studies, the formal order of accuracy must be stated, and that iterative convergence is achieved with at least three orders of magnitude decrease in normalized residuals [source]. The policy also recommends that the method for discretization error estimation is the Richardson Extrapolation (RE) method [32]. The Grid Convergence Index (GCI) method, based on the RE method, is described in the policy statement and is used to evaluate the grid convergence and data gathered from the meshes generated by the author for the use in this thesis [10].

\section{E.1 Convergence Study Setup}

Eight meshes were generated with different cell spacing, three were chosen for analysis based on their grid reduction values. Five test points were chosen over the grid to analyze the convergence of the grids. Two of the points are at the cusps of the magnets, two are along the centerline, and the last is in the middle of the field. Figure E.1 illustrates the magnetic field solution and the five test points analyzed. 


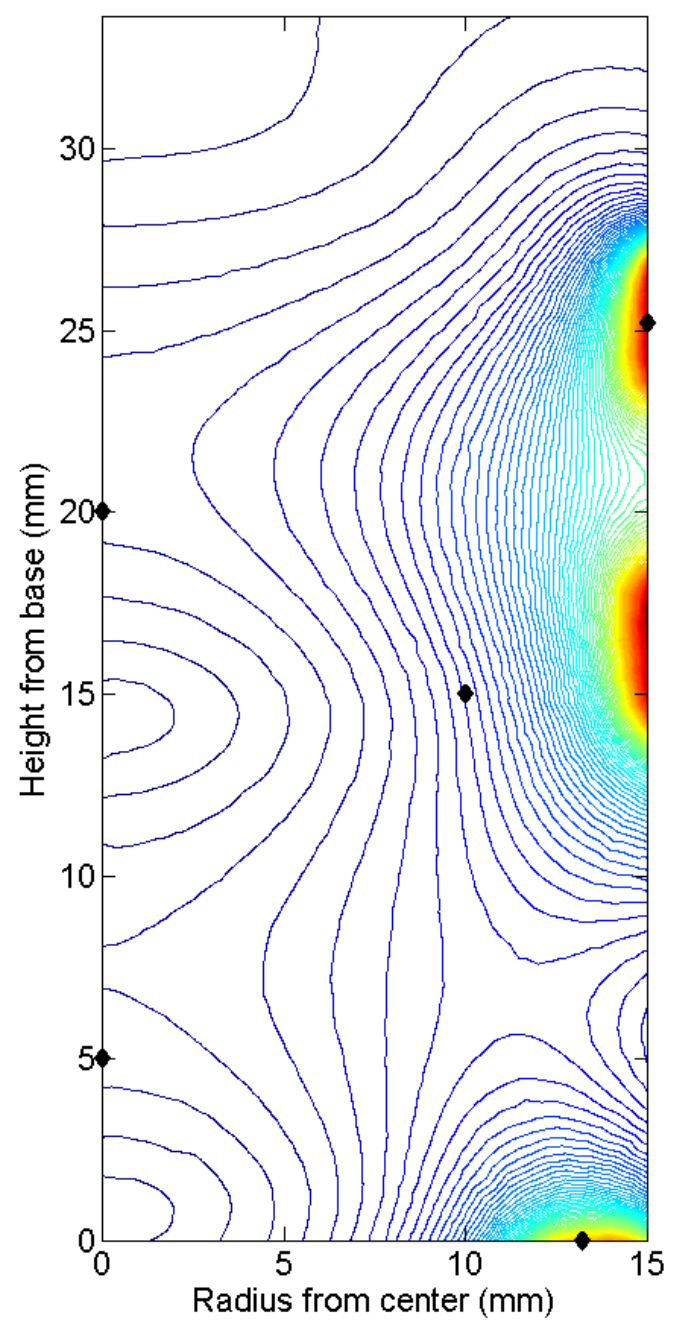

Figure E.1: Grid Solution Space with Test Points

Table E. 1 describes the grids used in this analysis, including the values at the five points in the mesh chosen as test points for convergence. Also listed in the table are the number of elements in each grid and the refinement values between the three grid sizes. 
Table E.1: Grid Convergence Analysis Setup

\begin{tabular}{|l|c|c|c|c|c|}
\hline & $\begin{array}{l}\text { Point 1 } \\
{[15,25.2]}\end{array}$ & $\begin{array}{l}\text { Point 2 } \\
{[13.2,0]}\end{array}$ & $\begin{array}{l}\text { Point 3 } \\
{[0,5]}\end{array}$ & $\begin{array}{l}\text { Point 4 } \\
{[0,20]}\end{array}$ & $\begin{array}{l}\text { Point 5 } \\
{[10,15]}\end{array}$ \\
\hline$N_{1}$ & 337032 & & & & \\
\hline$N_{2}$ & 81925 & & & & \\
\hline$N_{3}$ & 33788 & & & & \\
\hline$r_{21}$ & 2.03 & & & & \\
\hline$r_{32}$ & 1.56 & & & & \\
\hline$\phi_{1}(\mathrm{G})$ & 2892.79 & 2249.19 & 147.39 & 187.34 & 436.02 \\
\hline$\phi_{2}(\mathrm{G})$ & 2941.68 & 2240.64 & 147.63 & 187.10 & 436.46 \\
\hline$\phi_{3}(\mathrm{G})$ & 2914.35 & 2313.34 & 145.25 & 183.29 & 464.83 \\
\hline
\end{tabular}

The representative cell size $h$ is the average cell size across the domain and is defined by

$$
h=\left[\frac{1}{N} \sum_{i=1}^{N}\left(\Delta A_{i}\right)\right]^{1 / 2}
$$

where $\Delta A_{i}$ is the area of the $i^{t h}$ cell and $N$ is the total number of cells used in the computations. With the domain area known and the number of cells specified by parameters in FEMM, the value of $h$ can be calculated for each grid.

The grid refinement factor $r$ is defined as the ratio of the representative cell sizes between a coarse and fine grid, with $h_{1}<h_{2}<h_{3}, r_{21}=h_{2} / h_{1}$ and $r_{32}=h_{3} / h_{2}$. According to the policy, it is desirable that these values be greater than 1.3 and that the refinement of the grids be done systematically for the most accurate results [10]. The grid refinement factors are listed in the Setup Table E.1.

Representative values $\phi$ were chosen for the convergence analysis. The $\phi$ values were the magnetic field strength at five different points across the computational domain. The 
location of the points and the magnetic field strengths are also specified in the Setup Table E.1.

\section{E.2 Convergence Analysis}

It is necessary to calculate the apparent order $p$ of the convergence using the equations

$$
\begin{aligned}
& p=\frac{1}{\ln \left(r_{21}\right)}|\ln | \varepsilon_{32} / \varepsilon_{21}|+q(p)| \\
& q(p)=\ln \left(\frac{r_{21}^{p}-s}{r_{32}^{p}-s}\right) \\
& s=1 \cdot \operatorname{sign}\left(\varepsilon_{32} / \varepsilon_{21}\right)
\end{aligned}
$$

The values of $\varepsilon$ are the difference between the values of $\phi$ where $\varepsilon_{32}=\phi_{3}-\phi_{2}$ and $\varepsilon_{21}=\phi_{2}-\phi_{1}$. Equation E.2a can be solved using fixed-point iteration with an initial guess of $p$ with $q(p)=0$. Negative values of $s$ indicate oscillatory convergence towards a zero grid spacing solution.

The extrapolated values of $\phi$ are then calculated by

$$
\phi_{\text {ext }}^{21}=\left(r_{21}^{p} \cdot \phi_{1}-\phi_{2}\right) /\left(r_{21}^{p}-1\right)
$$

and similarly for $\phi_{\text {ext }}^{32}$.

The error estimates can then be calculated knowing the values of $\phi$ and their extrapolated values. The Approximate relative error is

$$
e_{a}^{21}=\left|\frac{\phi_{1}-\phi_{2}}{\phi_{1}}\right|
$$

and the Extrapolated relative error is

$$
e_{e x t}^{21}=\left|\frac{\phi_{e x t}^{21}-\phi_{1}}{\phi_{e x t}^{21}}\right|
$$

and the Fine-Grid Convergence Index (GCI) is

$$
G C I_{\text {fine }}^{21}=\frac{1.25 e_{a}^{21}}{r_{21}^{p}-1}
$$


The extrapolated relative error is a measure of how far away the most refined grid's solution is from the extrapolated solution. The GCI is a measure of how far the computed value is away from the asymptotic numerical solution value. It is desirable to have the values of error and CGI less than $1 \%$.

\section{E.3 Convergence Analysis Results}

A grid convergence study was performed based on the Richardson Extrapolation and Grid Convergence Index using the values from Table E.1. The magnetic field strength solution for the three grids is plotted in Figures E.2a and E.2b below for two of the test points. Also shown are the extrapolated solution values and a point marking the grid spacing that was used throughout the thesis. The spacing was used because it maintained low error and had a shorter run time when many grids were created and analyzed throughout the thesis.

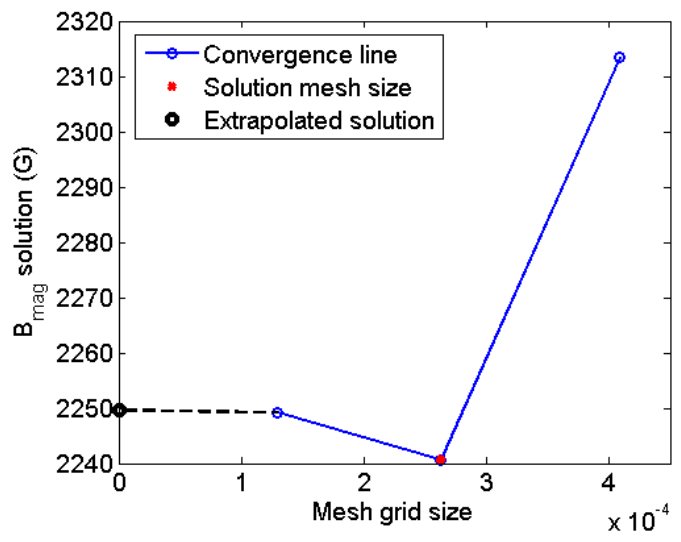

(a) Convergence at Point $2[13.2,0]$

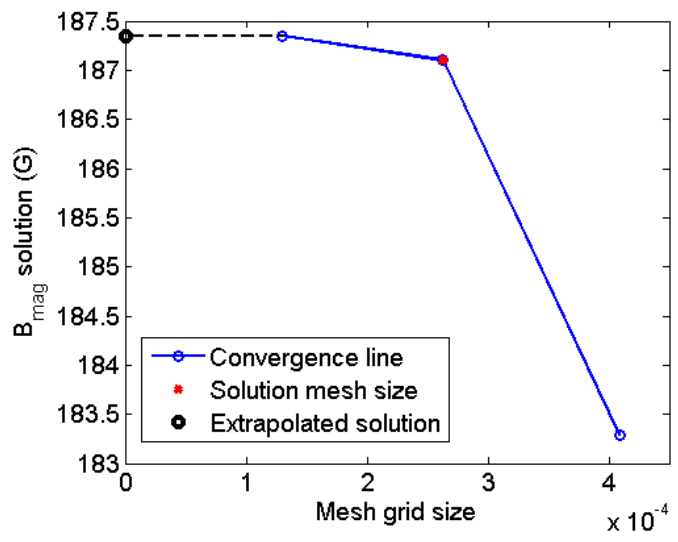

(b) Convergence at Point $4[0,20]$

Figure E.2: Grid Convergence Study Results of two test points Figure E.2a shows the solution and extrapolation at Point $2[13.2,0]$ while Figure E.2b shows the solution and extrapolation at Point $4[0,20]$

From the figures, it is easy to see the change in the solution as the mesh size is refined and that the extrapolated value is similar to the finest grid and the solution set grid. Table E.2 details the results of the grid convergence study for all five points in the domain. 
Table E.2: Grid Convergence Analysis Results

\begin{tabular}{|l|c|c|c|c|c|}
\hline & $\begin{array}{l}\text { Point 1 } \\
{[15,25.2]}\end{array}$ & $\begin{array}{l}\text { Point 2 } \\
{[13.2,0]}\end{array}$ & $\begin{array}{l}\text { Point 3 } \\
{[0,5]}\end{array}$ & $\begin{array}{l}\text { Point 4 } \\
{[0,20]}\end{array}$ & $\begin{array}{l}\text { Point 5 } \\
{[10,15]}\end{array}$ \\
\hline$\phi_{1}(\mathrm{G})$ & 2892.79 & 2249.19 & 147.39 & 187.34 & 436.02 \\
\hline$\phi_{2}(\mathrm{G})$ & 2941.68 & 2240.64 & 147.63 & 187.10 & 436.46 \\
\hline$\phi_{3}(\mathrm{G})$ & 2914.35 & 2313.34 & 145.25 & 183.29 & 464.83 \\
\hline$p$ & 0.67 & 4.64 & 5.07 & 6.30 & 9.42 \\
\hline$\phi_{\text {ext }}^{21}$ & 2812.55 & 2249.53 & 147.39 & 187.35 & 436.02 \\
\hline$e_{a}^{21}$ & $1.6902 \%$ & $0.3805 \%$ & $0.1590 \%$ & $0.1316 \%$ & $0.1019 \%$ \\
\hline$e_{\text {ext }}^{21}$ & $2.8528 \%$ & $0.0148 \%$ & $0.0045 \%$ & $0.0015 \%$ & $0.0001 \%$ \\
\hline$G C I_{\text {fine }}^{21}$ & $3.4671 \%$ & $0.0185 \%$ & $0.0057 \%$ & $0.0019 \%$ & $0.0002 \%$ \\
\hline
\end{tabular}

The local order of accuracy $p$ ranges from 0.67 to 9.42 with an average of 5.22 and three of the five test points exhibited oscillatory convergence through the grid refinements. The results show almost all of the points chosen for analysis have a total error of less than $1 \%$, and most significantly less. The one point of concern is that of the upper magnetic cusp where the total error was $3.4671 \%$. This translates to an uncertainty in the magnetic cusp strength of \pm 100.2948 Gauss.

The results of this grid convergence study demonstrate confidence in the data collected from FEMM used throughout this thesis. 


\section{Appendix F}

\section{LANGMUIR PROBE TESTING AND ANALYSIS}

The plasma parameters of the thruster during operation were determined through the use of a Langmuir probe. The methods and processes for analyzing Langmuir probe sweeps are well known [7], this section will summarize and describe the specifics to the Langmuir analysis for this thesis.

The Langmuir probe was a $0.5 \mathrm{~mm}$ diameter tungsten wire protruding $1.65 \mathrm{~mm}$ from a ceramic sheath. The tungsten lead was attached to a copper wire, fed through the vacuum chamber, through a $100 \Omega$ resistor, and to the output terminal of a Kepco-BOP $100 \mathrm{~W}$ power supply. This power supply is a bipolar power supply capable of producing potentials of $\pm 100 \mathrm{~V}$, ideal for the range of values desired for Langmuir sweeps. The voltage of the supply was read through a voltage divider and a NI-6008 DAQ, the current was determined by using a Voltmeter to measure the voltage drop across the resistor. The data was acquired through LabVIEW and processed in MATLAB.

Once the throttle point of the thruster was set, the power supply was turned on and adjusted to $-50 \mathrm{~V}$ and the current reading was recorded. The voltage was increased small amounts and the current recorded until reaching a potential near $0 \mathrm{~V}$ on the power supply. Near this value, there will be significant changes to the current for very small changes to the voltage. The voltage step size must be reduced to adequately capture the data. The voltage step size can be increased again when past the area of high slope. The voltage trace is continued until reaching $+100 \mathrm{~V}$. A sample output of the raw data from one of the sweeps is shown in Figure F.1. 


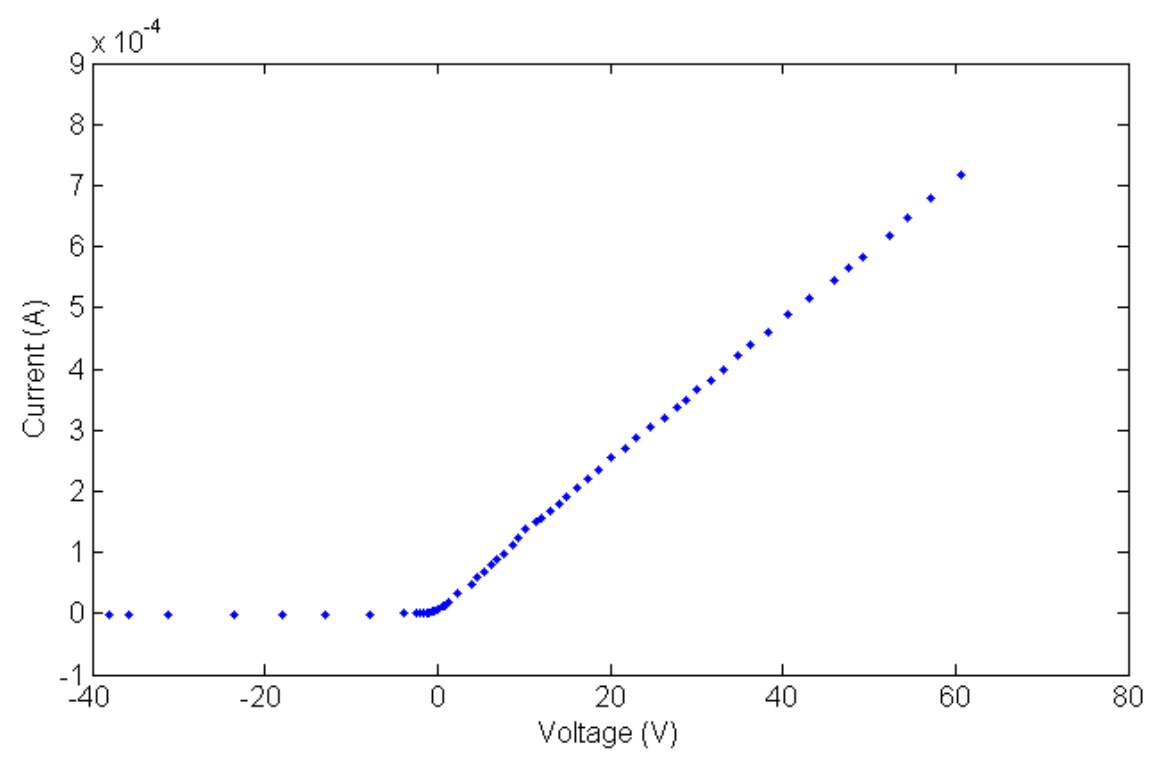

Figure F.1: Langmuir Probe Sweep Raw Data (Note the higher density of data points near $0 \mathrm{~V}$ and above)

When done by hand, this process can be rather time consuming. Significant effort was made to digitize and automate the process of data acquisition and analysis, but problems with equipment and the lack of specific electrical components prevented that goal. Due to the increased amount of time required, it was important to not overheat the thruster when running the Langmuir sweeps. While most of the data could be completed for the full sweep, some needed to be stopped short and only the plasma temperature from those sweeps were considered valid. Future work includes building a system that can quickly and accurately run the Langmuir sweep over the entire range of voltages to record the data points.

\section{F.1 Langmuir Probe Results}

From the raw data, the floating potential $V_{f}$ and the ion saturation current $I i_{\text {sat }}$ can be determined. The floating potential is determined by the point where the ion and electron 
currents balance and the current through the resistor reads zero. Following with this sample data set, the floating potential was determined to be $-1.07 \mathrm{~V}$.

To calculate the ion saturation current, it is necessary to find the slope of the linear fit to the linear region below the floating potential. The equation for the line is determined and the ion saturation value is calculated by evaluating the function for the line at the floating potential. This is exemplified by the equation

$$
I i_{\text {sat }}=m_{\text {slope }} V_{f}+b_{\text {intercept }}
$$

where $m_{\text {slope }}$ and $b_{\text {intercept }}$ are the slope and intercept of the linear fit to the lower region of the sweep. Figure F.2 demonstrates finding the bounds of the ion saturation region and the ion saturation current.

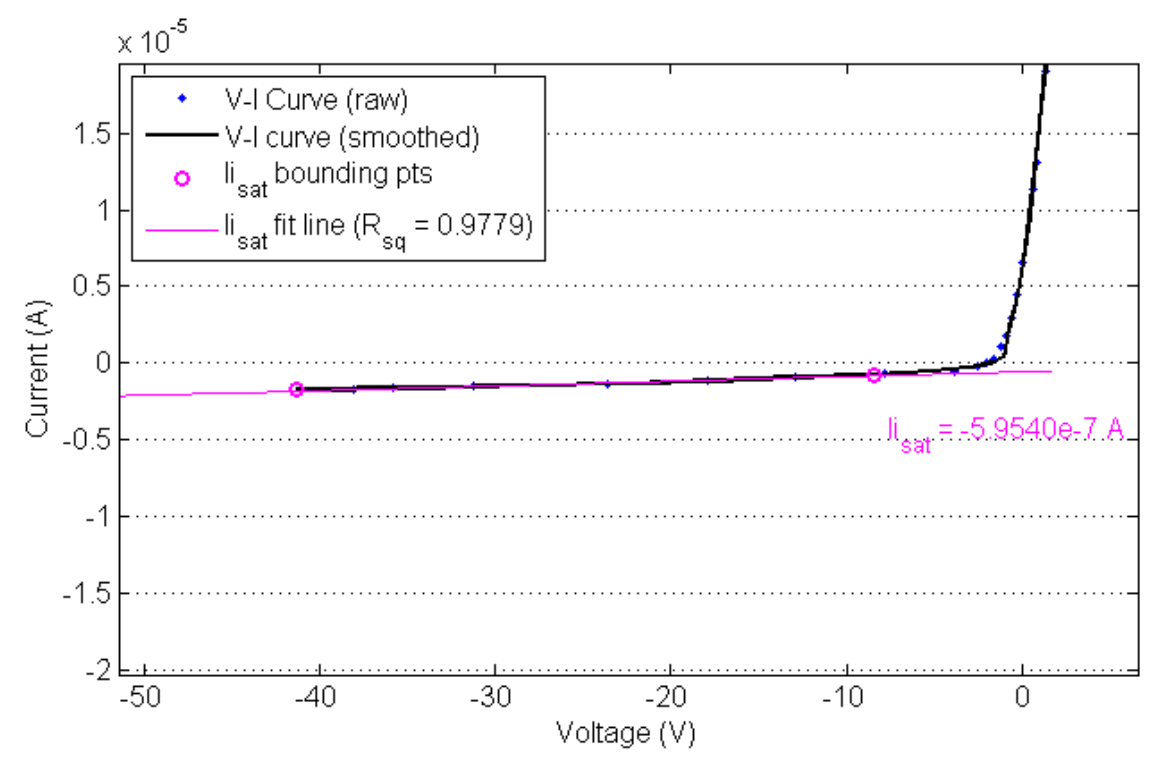

Figure F.2: Langmuir Probe Analysis of Ion Saturation Current

The magnitude of the ion saturation current is added to the raw data and raises the values of the raw data set to have few remaining negative terms. With the data set adjusted, the $\log$ is taken of the current terms. The resulting output is shown in Figure F.3. 


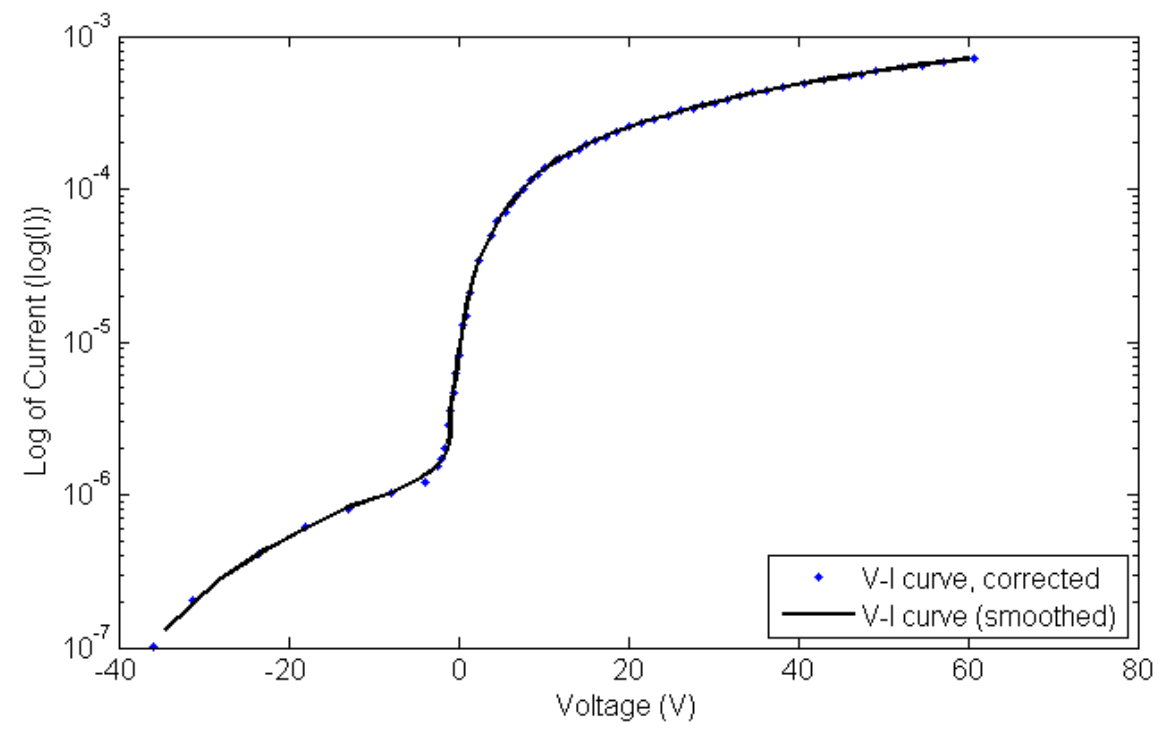

Figure F.3: Langmuir Sweep of the Log of Current versus Voltage

In this figure, there are two distinct linear regions: one near the floating potential and one at the highest potential region. Similar to determining the ion saturation current, the bounds of the linear regions are determined and an exponential fit is calculated between the points. The results of the bounds and fits are shown in Figure F.4.

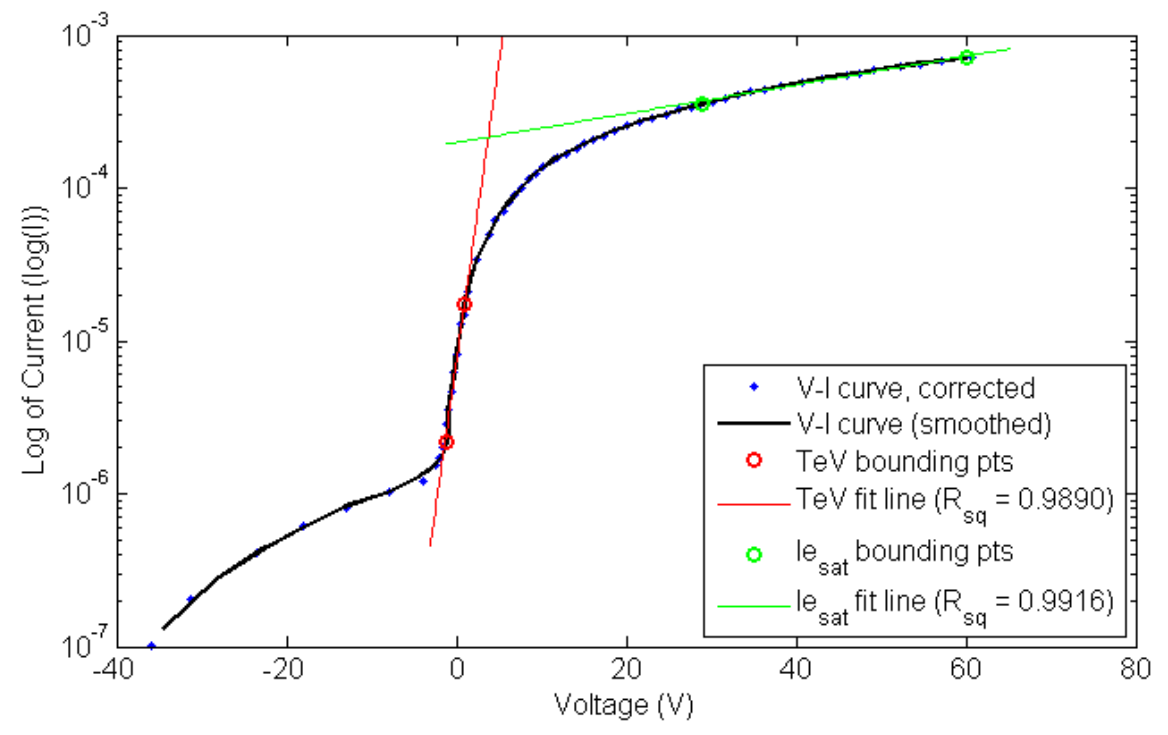

Figure F.4: Langmuir Probe Analysis of Electron Temperature and Electron Saturation Current 
The inverse of the slope of the electron temperature linear region is the electron temperature $T_{e V}$. The intersection between the electron temperature and saturation lines yields the plasma potential $\phi$. In this test case, the electron temperature was determined to be $1.11 \mathrm{eV}$ and the plasma potential was determined to be $3.76 \mathrm{~V}$. The results of the Langmuir analysis is summarized in the Figure F.5.

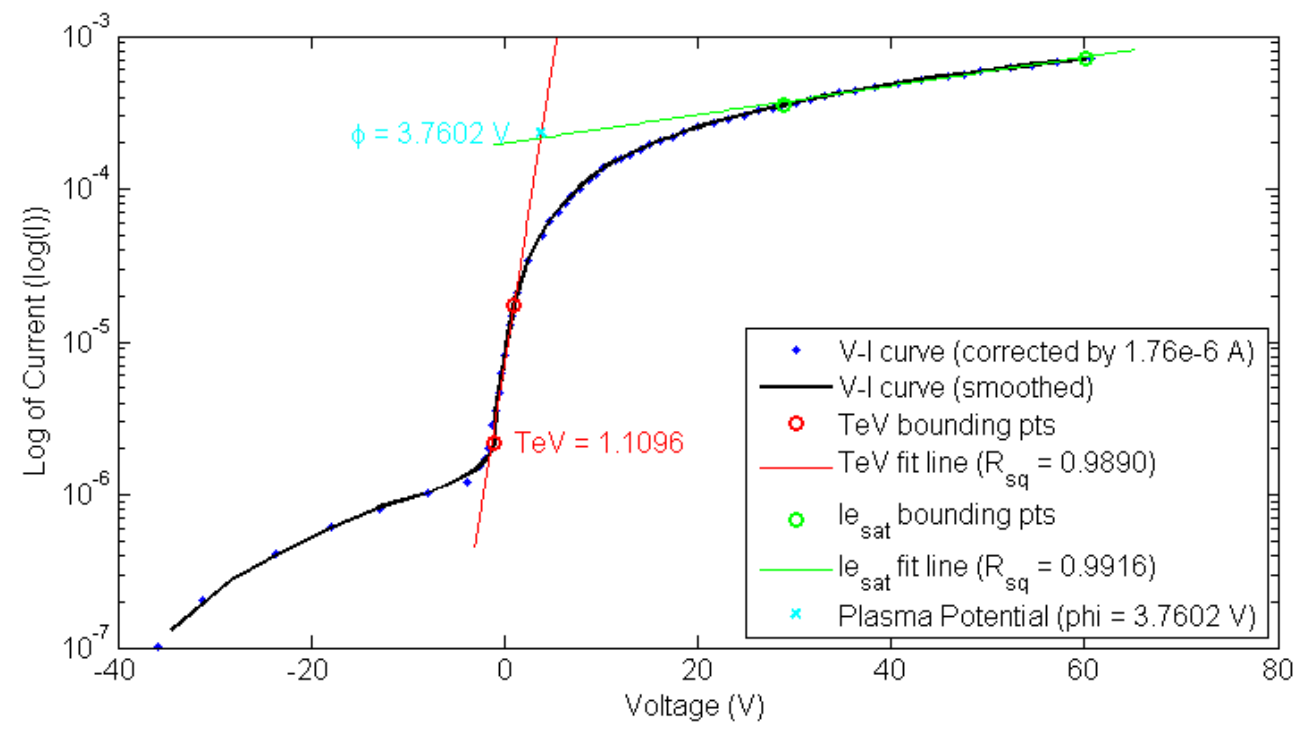

Figure F.5: Completed Langmuir Probe Analysis

The last bit of important information is the calculation of the plasma density. There is controversy over the best method to do this as there is systematic error involved with the calculations and different methods will yield drastically different values. In this analysis, the calculation of the plasma density is determined from the measured ion saturation current and the equations

$$
\begin{aligned}
n_{i} & \approx \frac{-2 I_{i}}{e A_{\text {probe }} v_{\text {Bohm }}} \\
v_{\text {Bohm }} & =\sqrt{\frac{e T_{e V}}{M_{i}}}
\end{aligned}
$$


where $A_{\text {probe }}$ is the area of the exposed probe and $v_{B o h m}$ is the Bohm velocity calculated from the electron temperature and the propellant ion mass. In this analysis, the ion density, and the electron density by quasi-neutrality, was calculated as $3.97 \times 10^{15} \mathrm{n} / \mathrm{m}^{3}$.

This analysis is performed outside the discharge chamber on the beam plasma when the beam is not energized. There are known differences between the plasma in the two regions [4], but there was no way to measure inside the discharge chamber to determine the perfect relationship between the two plasmas. The Langmuir analysis performed on the plasma region outside the discharge chamber are estimated as the discharge chamber plasma values for the work presented in this thesis. 